Flat and round singularity theory

Mostafa Salarinoghabi 



\section{Mostafa Salarinoghabi}

\section{Flat and round singularity theory}

Doctoral dissertation submitted to the Instituto de Ciências Matemáticas e de Computação - ICMC-USP, in partial fulfillment of the requirements for the degree of the Doctorate in Science - Mathematics. FINAL VERSION

Concentration Area: Mathematics

Advisor: Prof. Dr. Farid Tari

USP - São Carlos

May 2016 
Ficha catalográfica elaborada pela Biblioteca Prof. Achille Bassi e Seção Técnica de Informática, ICMC/USP, com os dados fornecidos pelo(a) autor(a)

Salarinoghabi, Mostafa
Flat and round singularity theory /
Mostafa Salarinoghabi; orientador Farid Tari. -
São Carlos - SP, 2016.
136 p.
Tese (Doutorado - Programa de Pós-Graduação em
Matemática) - Instituto de Ciências Matemáticas e de
Computação, Universidade de São Paulo, 2016.
1. bifurcations. 2. evolutes. 3. plane curves.
4. inflections. 5. vertices. 6. singularities. I.
Tari, Farid, orient. II. Título.


Data de Depósito:

Assinatura:

\title{
Mostafa Salarinoghabi
}

\section{A teoria da singularidade plana e redonda}

\begin{abstract}
Tese apresentada ao Instituto de Ciências Matemáticas e de Computação - ICMC-USP, como parte dos requisitos para obtenção do título de Doutor em Ciências - Matemática. VERSÃO REVISADA
\end{abstract}

Área de Concentração: Matemática

Orientador: Prof. Dr. Farid Tari 

This thesis dedicated to my parents

for their endless love, support and encourangment,

$$
\text { and }
$$

to Pouya

who leads me through the valley of darkness with light of hope and support. 

First and foremost I want to thank my advisor prof. Farid Tari. It has been an honor to be his Ph.D. student. I appreciate all his contributions of time and ideas, to make my Ph.D. experience productive and stimulating. The joy and enthusiasm he has for his research was contagious and motivational for me, even during tough times in the Ph.D. pursuit.

Besides my advisor, I would like to thank Dr. Masaru Hasegawa for his patience and his useful helps. Also I want to thank all ICMC members (staffs and faculties), specially prof. Maria Aparecida Soares Ruas, prof. Ali Tahzibi and prof. Behrooz Mirzaii. Also I would like to thank "CAPES" for their financial support during my Ph.D. studies.

Special mention goes to my dear friends in particular, I am grateful to Pouya Mehdipour, Jorge Luiz Deolindo, Thiago Henrrique de Freitas, Thales Maier de Souza, Salimeh Yasaie, Fatemeh Yeganeh Mokari, Patrícia Tempesta, Martín Barajas Sichacá, Mona Mehdipour, Nelson Antonio Silva, Victor Simões Barbosa, Ginnara Mexia Souto, Maria Carolina Zanardo, Iman ghodrati and the other friends for your understanding and encouragement in my many, many moments of crisis. Your friendship makes my life a wonderful experience. I can not list all the names here, but you are always on my mind.

I would like to thank Pouya's parents, Jorge's family and sincerely thanks to my family: my beloved parents and to my brothers Mahdi and Alireza, my sister Taravat, my brother's wife Somayeh and my sister's husband Hosein for supporting me spiritually throughout writing this thesis and my life in general.

Finally, I would like to thank the jury members: prof. Fábio Scalco Dias, prof. Bruna Oréfice Okamoto and prof. Marcos Craizer for their time, interest, and valuable comments. 

"Where there is matter, there is geometry." (Johannes Kepler) 



\section{RESUMO}

SALARINOGHABI, M. . Flat and round singularity theory. 2016. 136 f. Doctoral dissertation (Doctorate Candidate in Science - Mathematics) - Instituto de Ciências Matemáticas e de Computação (ICMC/USP), São Carlos - SP.

Propomos nesta tese um método para estudar deformações de curvas planas que leva em consideração a geometria delas, bem como as suas singularidades. Consideramos em detalhes os fenômenos locais que ocorrem genericamente em famílias de curvas com dois parâmetros. Obtemos informações sobre as inflexões e vértices que aparecem nas curvas deformadas. Obtemos também as configurações das evolutas das curvas e das suas deformações e aplicamos os nossos resultados nas projeções ortogonais de curvas espaciais. Finalmente, consideramos o perfil de uma superfície regular no espaço Euclidiano $\mathbb{R}^{3}$. O perfil é a imagem do conjunto singular de uma projeção ortogonal da superfície, esta é uma curva plana e pode ter singularidades. Estudamos as alterações na geometria do perfil quando a direção de projeção muda localmente na esfera unitária.

Palavras-chave: bifurcações, evolutas, curvas planas, inflexões, vértices, singularidades. 



\section{ABSTRACT}

SALARINOGHABI, M. . Flat and round singularity theory. 2016. 136 f. Doctoral dissertation (Doctorate Candidate in Science - Mathematics) - Instituto de Ciências Matemáticas e de Computação (ICMC/USP), São Carlos - SP.

We propose in this thesis a way to study deformations of plane curves that take into consideration the geometry of the curves as well as their singularities. We deal in details with local phenomena that occur generically in two-parameter families of curves. We obtain information on the inflections and vertices appearing on the deformed curves. We also obtain the configurations of the evolutes of the curves and of their deformations, and apply our results to orthogonal projections of space curves. Finally, we consider the profile (outline, apparent contour) of a smooth surface in the Euclidian 3-space. This is the image of the singular set of an orthogonal projection of the surface. The profile is a plane curve and may have singularities. We study the changes in the geometry of the profile as the direction of projection changes locally in the unit sphere.

Key-words: bifurcations, evolutes, plane curves, inflections, vertices, singularities. 

INTRODUCTION . . . . . . . . . . . . . . . . . . . . 17

PRELIMINARY . . . . . . . . . . . . . . . . . . . . . 21

$2.1 \quad$ Germs of smooth mappings . . . . . . . . . . . . . . . . . . . . 21

2.1.1 Singularities of germs of smooth mappings . . . . . . . . . . . . . . . 22

$2.2 \quad$ Mather groups and their tangent space . . . . . . . . . . . . . 22

2.2.1 Tangent spaces to the $\mathcal{G}$-orbits . . . . . . . . . . . . . . . . . 24

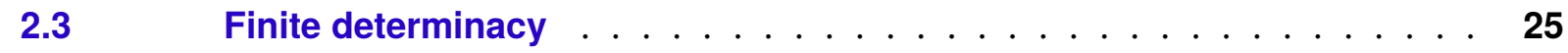

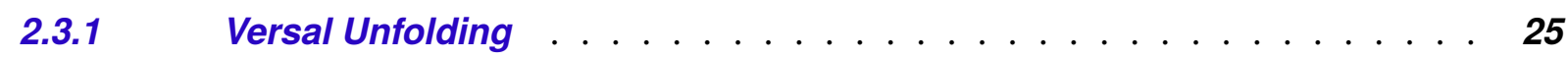

2.4 Germs of functions . . . . . . . . . . . . . . . . . . 27

2.4.1 Discriminant and bifurcation sets . . . . . . . . . . . . . . 29

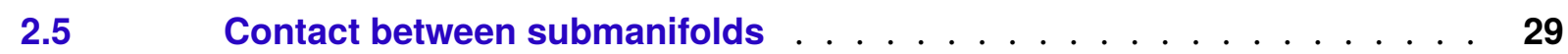

2.5.1 The family of height functions . . . . . . . . . . . . . . . . 30

2.5.2 Family of distance squared functions . . . . . . . . . . . . . . . 31

2.5.3 Family of projections into hyperplanes . . . . . . . . . . . . . . 33

$2.6 \quad$ Divergent diagrams . . . . . . . . . . . . . . . 34

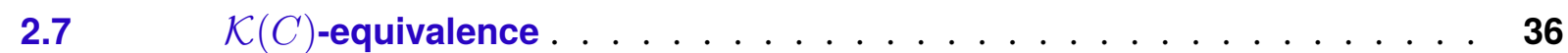

$3 \quad$ FLAT AND ROUND SINGULARITY THEORY OF PLANE CURVES . . . . . 39

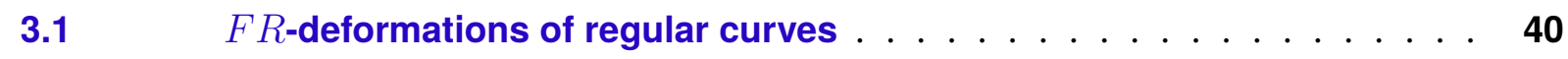

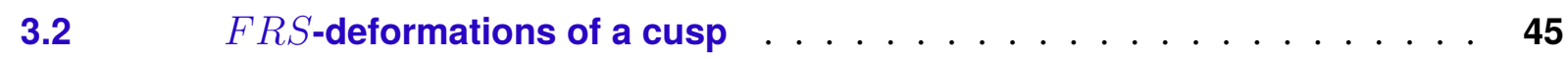

3.3 $F R S$-deformations of the ramphoid cusp $\ldots \ldots \ldots \ldots$

$4 \quad$ VERTICES AND EVOLUTES OF PROJECTIONS OF SPACE CURVES . . . 61

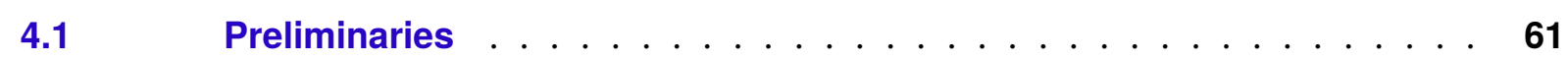

$4.2 \quad$ Regular projections . . . . . . . . . . . . . . . 63

4.2.1 Evolute of projections of space curves . . . . . . . . . . . . . 63

4.2.2 Projections of space curves and their contact with cylinder . . . . . . 71

$4.3 \quad$ Singular projections . . . . . . . . . . . . . 75

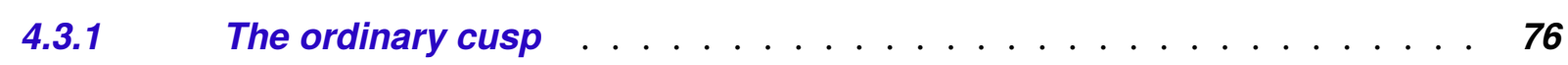

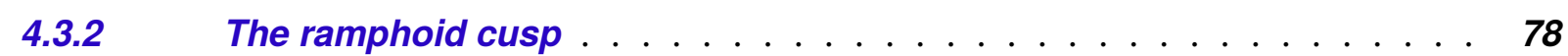

5 THE GEOMETRY OF ORTHOGONAL PROJECTION OF SURFACES IN $\mathbb{R}^{3} \quad 85$

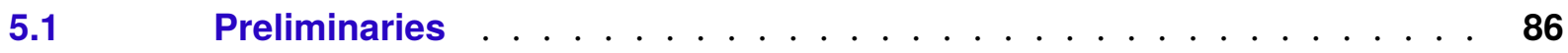




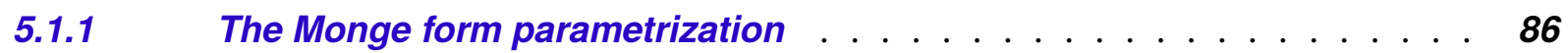

5.1.2 The modified family of orthogonal projections . . . . . . . . . . . . . 88

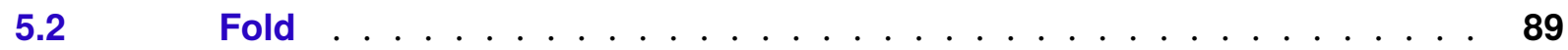

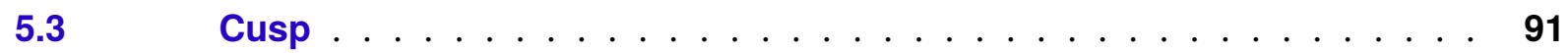

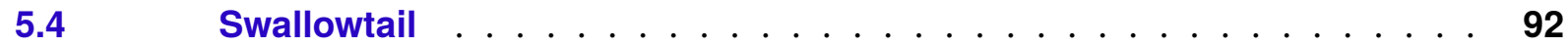

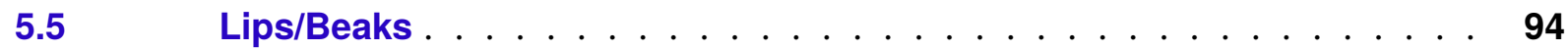

5.5.1 Evolute of the profile at a lips/beaks singularity . . . . . . . . . . 101

$5.6 \quad$ Butterfly . . . . . . . . . . . . . . . . . . . . . 104

$5.7 \quad$ Goose . . . . . . . . . . . . . . . . . . . . 107

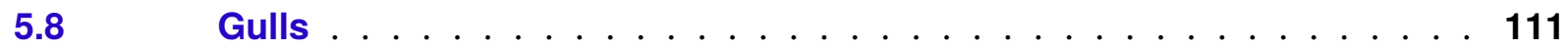

5.8.1 $A_{4}$-contact of surface with cylinder at the cusp of Gauss point . . . . . 112

5.8.2 Stratification of the family of modified orthogonal projections at a gulls singularity . . . . . . . . . . . . . . . . . 116

$5.9 \quad$ On $F R S$-theory for discriminant of map germs $\mathbb{R}^{2} \rightarrow \mathbb{R}^{2} \ldots \ldots 119$

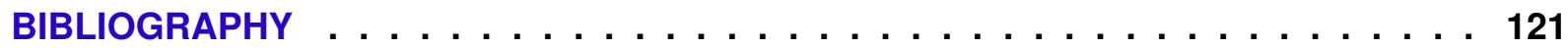

APPENDIX A MAPLE CODES IN THE PROOF OF THEOREM $4.3 \ldots 125$

APPENDIX B $\quad$ MAPLE CODES IN THE PROOF OF THEOREM $4.4 \ldots \ldots$

APPENDIX C $\quad$ MAPLE CODES IN THE PROOF OF THEOREM $5.4 \ldots \ldots$

APPENDIX D MAPLE CODES IN THE PROOF OF THEOREM $5.7 \ldots \ldots$ 


\section{1}

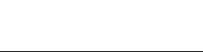

INTRODUCTION

In the first part of this thesis, we consider smooth $\left(C^{\infty}\right)$ parametrised plane curves (for a study of curves given by equations see $[10,15])$. Consider a plane curve with a cusp singularity. If we are interested (locally) only in the singularity of the curve and the way it bifurcates, we can reparametrise the curve and change coordinates in the plane (so we have the action of the group $\mathcal{A}$ of germs of changes of coordinates in the source and target on map-germs $(\mathbb{R}, 0) \rightarrow\left(\mathbb{R}^{2}, 0\right)$ ). An $\mathcal{A}$-model for the cusp curve is $\left(t^{2}, t^{3}\right)$ and a model of an $\mathcal{A}_{e}$-versal deformation of this singularity is $\left(t^{2}, t^{3}+u t\right)$; see Figure 1.1 .

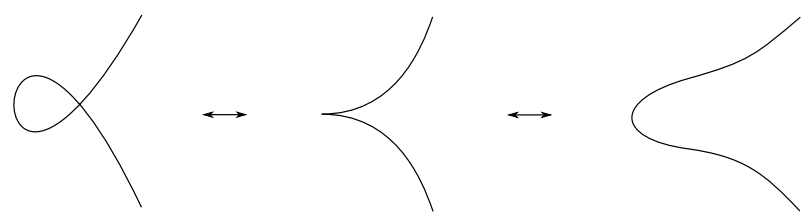

Figure 1.1 - Generic bifurcations of a cusp singularity.

We may also be interested in the geometry of the plane curve, such as its inflections and its vertices. In this case, we are allowed only Euclidean motions in the plane as diffeomorphisms destroy the geometry of the curve (but preserve its singularities). We can of course reparametrise the curve.

Several question can be asked. What is the meaning of a generic deformation of the curve? How many inflections and vertices appear in a generic deformation? What is the evolute of the singular plane curve and how does it bifurcate in generic families of curves? Is there a theory that can give a model of the deformation of the singularity of the curve as well as taking into consideration its geometry?

Singular plane curves can be related to regular curves. For instance, an orthogonal projection of a regular space curve to a plane is singular if the direction of projection is tangent to the space curve. Geometric information on the projected curve provide geometric information about the 
space curve itself $([12,14,26,34])$.

In [14], Nuño-Ballesteros and Dias proposed a way to find models of the singularities of plane curves as well as their contact with lines at the singular point of the curve. (The contact with lines captures the inflections concentrated at the singular point of the curve.) They did this by classifying germs of divergent diagrams $(f, \alpha): \mathbb{R}, 0 \stackrel{f}{\longleftarrow} \mathbb{R}, 0 \stackrel{\alpha}{\longrightarrow} \mathbb{R}^{2}, 0$ up to smooth changes of coordinates. An alternative approach to using divergent diagrams is proposed in [26]. A classification of submersions up to smooth changes of coordinates in the source preserving an $\mathcal{A}$-model of the singularity of the curve is given in [26]. The group acting is a Damon geometric subgroup, so the classification tools of singularity theory can be used. Wall introduced in [32] an equivalence relation which takes into consideration the tangent cone of the curve. He used invariants of the curve to classify the singularities. The various proposed methods $([14,26,32])$ can handled a single curve. However, finding a theory that explains the deformations of the singularity of the curve in families as well as the changes in its geometry (appearance and location of the inflections and vertices) is still an open problem. (Of course the question is not restricted to curves and is valid for submanifolds of $\mathbb{R}^{n}$.) A study of the particular case of families of projections of space curves is carried out [26]. The way the singularities and the inflections of the projected curve bifurcate when the direction of projections varies locally in the unit sphere is given. The bifurcations in the dual of the projected curve are also determined in [26].

From Proposition 3.3, any deformation of a singular curve results in a non-versal deformation of the family of distance squared functions on the curve. This indicates that the theory we are seeking may not come from an action of some subgroup of the contact group $\mathcal{K}$ on the set of germs functions/mappings on the curve.

We describe in this thesis a method for studying the geometry of deformations of singular plane curves, which we label $F R S$-deformation of plane curves ( $\mathrm{F}$ for flat, $\mathrm{R}$ for round and $\mathrm{S}$ for singular). When the curve is regular we label the deformations by $F R$-deformations. The method is similar to the one used to study bifurcations of vector fields and certain implicit differential equations (see for example [1, 11, 16, 27, 28, 29, 30]).

For two germs of $m$-parameter deformations $\gamma_{s}$ and $\eta_{u}$ of a (singular) plane curve, we define the concept of $F R S$-equivalence. Basically, $F R S$-equivalence means the instantaneous configurations of the curves $\gamma_{s}$ and $\eta_{k(s)}$ (including their singularities, vertices, inflections and their evolutes) are the same.

We also define the concept of $F R S$-generic families (case by case). The idea is to show that any two $F R S$-generic $m$-parameter families are $F R S$-equivalent. This allows us to obtain an $F R S$-model of $F R S$-deformations of a plane curve singularity. We do this in this thesis for all the codimension $\leq 2$ pheneomena and relate in each case the concept of $F R S$-genericity to that of transversality of the (multi-) jet extension map to a stratification of the (multi-) jet space.

After a brief introduction of some preliminary concepts in Chapter 2, In Chapter 3 we deal 
with regular curves at inflections of any finite order (\$3.1) and with the cusp (\$3.2) and ramphoid cusp (\$3.3) singularities. We define in each case the meaning of a generic family and obtain the conditions for two such families to be $F R S$-equivalent. We also obtain the configuration of the deformed curve and of its evolute. (We observe that in [13] are obtained formulae for the number of vertices and inflections of germs of singular plane curves and a relation between these numbers.) As one application of $F R S$-equivalence, in Chapter 4 we apply our results to orthogonal projections of space curves. Chapter 5 is devoted to profile of smooth surfaces in the Euclidian 3-space. This is the image of the singular set of an orthogonal projection of the surface. The profile is a plane curve and may have singularities. We study the changes in the geometry of the profile as the direction of projection changes locally in the unit sphere. Finally we extend $F R S$-theory for discriminant of map germs from $\mathbb{R}^{2}$ to $\mathbb{R}^{2}$. 

In this chapter we give some basic concepts from singularity theory. Mostly, we will use [6], [24] and [33] as references.

\subsection{Germs of smooth mappings}

Let $X$ and $Y$ be two subsets of $\mathbb{R}^{n}$ containing a point $p \in \mathbb{R}^{n}$. We say that $X$ is equivalent to $Y$ if there exists an open set $U \subset \mathbb{R}^{n}$ containing $p$ such that $X \cap U=Y \cap U$. This defines an equivalence relation among subsets of $\mathbb{R}^{n}$ containing the point $p$. The equivalence class of a subset $X$ is called the germ of $X$ at $p$ and is denoted by $(X, p)$.

Let $U$ and $V$ be two open subsets of $\mathbb{R}^{n}$ containing a point $p \in \mathbb{R}^{n}$, and let $f: U \rightarrow \mathbb{R}^{m}$ and $g: V \rightarrow \mathbb{R}^{m}$ be two smooth maps. We say that $f \sim g$ if there exists an open set $W \subset U \cap V$ containing $p$ such that $f=g$ on $W$, that is $\left.f\right|_{W}=\left.g\right|_{W}$.

The relation $\sim$ is an equivalence relation and a germ at $p$ of a smooth map is by definition an equivalent class under this equivalence relation. A map-germ at $p$ is denoted by $f:\left(\mathbb{R}^{n}, p\right) \rightarrow$ $\mathbb{R}^{m}$.

Sometimes we require that all the elements of the equivalence classes have the same value at $p$, say $q$. Then we write

$$
f:\left(\mathbb{R}^{n}, p\right) \rightarrow\left(\mathbb{R}^{m}, q\right)
$$

Suppose that $f:\left(\mathbb{R}^{n}, 0\right) \rightarrow\left(\mathbb{R}^{m}, q\right)$ be a map-germ at origin. The space of all such germs is denoted by $\mathcal{E}(n, m)$. When $m=1$, we denote $\mathcal{E}(n, 1)$ by $\mathcal{E}_{n}$. The set $\mathcal{M}_{n}=\left\{f \in \mathcal{E}_{n}: \quad f(0)=\right.$ $0\}$ is a maximal ideal of $\mathcal{E}_{n}$.

For a given positive integer $k$, the $k$ th-power of the maximal ideal $\mathcal{M}_{n}$ is denoted by $\mathcal{M}_{n}^{k}$. It is the set of germs of functions $f \in \mathcal{M}_{n}$ with zero partial derivatives of order less or equal to $k-1$ 
at the origin. We also have

$$
\mathcal{M}_{n}^{k}=\mathcal{E}_{n} \cdot\left\{x_{1}^{i_{1}} \ldots x_{n}^{i_{n}}: i_{1}+\cdots+i_{n}=k\right\}
$$

We denote by $\mathcal{M}_{n}^{k+1}$. $\mathcal{E}(n, m)=\mathcal{M}_{n, m}^{k+1}$ the set of map-germs $f:\left(\mathbb{R}^{n}, 0\right) \rightarrow\left(\mathbb{R}^{m}, 0\right)$ with vanishing partial derivatives of order less or equal to $k$ at the origin.

The $k$-jet space of smooth map-germs $\left(\mathbb{R}^{n}, 0\right) \rightarrow\left(\mathbb{R}^{m}, 0\right)$ is defined as

$$
J^{k}(n, m)=\mathcal{M}_{n} \cdot \mathcal{E}(n, m) / \mathcal{M}_{n}^{k+1} \cdot \mathcal{E}(n, m)
$$

The map $j^{k}: \mathcal{M}_{n} . \mathcal{E}(n, m) \rightarrow J^{k}(n, m)$ assigns to each map-germ $f$ its $k$ th-jet. An element in $J^{k}(n, m)$ corresponding to a map-germ $f \in \mathcal{M}_{n} \cdot \mathcal{E}(n, m)$ is denoted by $j^{k} f$. The set $J^{k}(n, m)$ can be identified with the set of polynomials of degree less than or equal to $k$, without the constant terms. Given $f \in \mathcal{M}_{n} . \mathcal{E}(n, m), j^{k} f$ is simply its Taylor polynomial of degree $k$ at the origin.

\subsubsection{Singularities of germs of smooth mappings}

Let $f: U \subset \mathbb{R}^{n} \rightarrow \mathbb{R}^{m}$ be a smooth map and denote by $d f: T U \rightarrow T \mathbb{R}^{m}$ its derivative map. The map $f$ is singular at $p \in U$ if the rank of the linear map

$$
(d f) p: \mathbb{R}^{n} \rightarrow \mathbb{R}^{m}
$$

is not maximal, that is, if $\operatorname{rank}(d f)_{p}<\min (n, m)$. The point $p$ is then said to be a singular point of $f$. Otherwise, we say that $f$ is non-singular at $p$ and $p$ is a regular point of $f$. The critical set of $f$, denoted by $\Sigma(f)$, is the set of singular points of $f$, that is,

$$
\Sigma(f)=\left\{p \in U \mid \operatorname{rank}(d f)_{p}<\min (n, m)\right\}
$$

The criminant of $f$, denoted by $C_{r}(f)$, is

$$
C_{r}(f)=\left\{p \in U \mid \operatorname{rank}(d f)_{p}<m\right\}
$$

When $n \geq m, C_{r}(f)=\Sigma(f)$, and when $n<m, C_{r}(f)=U$. The discriminant of $f$, denoted by $\Delta(f)$, is the image of $C_{r}(f)$ by $f$ :

$$
\Delta(f)=f\left(C_{r}(f)\right)
$$

\subsection{Mather groups and their tangent space}

Let $G$ be a Lie group and denote the tangent space of $G$ at $f$ by $T_{f} G$. 
Definition 2.1. Given a smooth manifold $M$ and a Lie group $G$, an action of $G$ over $M$ is a smooth map

$$
\begin{aligned}
& G \times M \rightarrow M \\
& (g, x) \mapsto g \cdot x,
\end{aligned}
$$

such that for any $g, h \in G$, and $x \in M, 1 . x=x$ and $g .(h . x)=(g h) . x$.

The action defines an equivalence relation in $G$. For $x, y \in M$ we say that $x \sim y$ if and only if there is $g \in G$ such that $y=g . x$. The orbit of $x$ is the equivalence class of $x$, which is equal to the set

$$
G . x=\{g \cdot x, g \in G\} .
$$

Theorem 2.1 ([24]). Let $G$ be a Lie group acting on a smooth manifold M. Then the orbits G.x, $x \in M$, are immersed submanifold of $M$.

Let $\mathcal{R}$ denotes the group of germs of all diffeomorphisms $\left(\mathbb{R}^{n}, 0\right) \rightarrow\left(\mathbb{R}^{n}, 0\right)$. This group is called the right group and acts smoothly on $\mathcal{M}_{n} \cdot \mathcal{E}(n, m)$ by

$$
h . f:=f \circ h^{-1} .
$$

The left group $\mathcal{L}$ of germs of all diffeomorphisms $\left(\mathbb{R}^{m}, 0\right) \rightarrow\left(\mathbb{R}^{m}, 0\right)$ acts smoothly on $\mathcal{M}_{n} \cdot \mathcal{E}(n, m)$ by

$$
k . f=k \circ f .
$$

The group $\mathcal{R}$ (resp. $\mathcal{L}$ ) is also called the group of changes of coordinates in the source (resp. target).

The group $\mathcal{A}:=\mathcal{R} \times \mathcal{L}$ acts smoothly on $\mathcal{M}_{n} \cdot \mathcal{E}(n, m)$, and the action is defined by

$$
(h, k) . f:=k \circ f \circ h^{-1} .
$$

Another interesting group, is the group of germs of diffeomorphisms $H:\left(\mathbb{R}^{n} \times \mathbb{R}^{m}, 0\right) \rightarrow$ $\left(\mathbb{R}^{n} \times \mathbb{R}^{m}, 0\right)$ such that $H(x, y)=(x, \bar{H}(x, y))$ with $\bar{H}(x, 0)=0$ for $x \in \mathbb{R}^{n}$ near the origin. We denote this group by $\mathcal{C}$. It acts smoothly on $\mathcal{M}_{n} . \mathcal{E}(n, m)$ by

$$
H . f(x):=H(x, f(x)), \quad H \in \mathcal{C}, \quad f \in \mathcal{M}_{n} . \mathcal{E}(n, m) .
$$

The group $\mathcal{C}$ is a normal subgroup of the contact group $\mathcal{K}$, where $\mathcal{K}$ is the group of germs of diffeomorphisms $H:\left(\mathbb{R}^{n} \times \mathbb{R}^{p}, 0\right) \rightarrow\left(\mathbb{R}^{n} \times \mathbb{R}^{p}, 0\right)$ which are written as $H(x, y)=(h(x), \bar{H}(x, y))$ such that $h \in \mathcal{R}$ and $\bar{H}(x, 0)=0$ for $x \in \mathbb{R}^{n}$ near to the origin. The action of $\mathcal{K}$ over $\mathcal{M}_{n} \cdot \mathcal{E}(n, m)$ is defined by

$$
H . f(x):=H\left(h^{-1}(x), f\left(h^{-1}(x)\right)\right), \quad H \in \mathcal{K}, \quad f \in \mathcal{M}_{n} . \mathcal{E}(n, m) .
$$

The groups $\mathcal{R}, \mathcal{L}, \mathcal{A}, \mathcal{C}$ and $\mathcal{K}$ are called the Mather groups. 


\subsubsection{Tangent spaces to the $\mathcal{G}$-orbits}

The tangent space at $f \in \mathcal{E}(n, p)$, denoted by $\theta_{f}$, is defined as the $\mathcal{E}_{n}$ - module of vector fields along $f$. Therefore elements of $\theta_{f}$ are of the form $\xi:\left(\mathbb{R}^{n}, 0\right) \rightarrow T \mathbb{R}^{p}$ satisfying $\pi_{p} \circ \xi=f$, where $\pi_{p}: T \mathbb{R}^{p} \rightarrow \mathbb{R}^{p}$ is the projection of the tangent fiber $T \mathbb{R}^{p}$ of $\mathbb{R}^{p}$ to $\mathbb{R}^{p}$.

Let $\mathcal{G}$ be a subgroup of $\mathcal{K}$ and $\phi:(-\epsilon, \epsilon) \times \mathbb{R}^{n+p}, 0 \rightarrow \mathbb{R}^{n+p}, 0$ is a curve in $\mathcal{G}$ such that $\phi_{0}$ is the identity element of $\mathcal{G}$. Differentiating this map gives the vector field

$$
\left.z \rightarrow \frac{\partial \phi}{\partial t}(t, z)\right|_{t=0}
$$

We denote the set of all such vector fields by $L \mathcal{G}$ and call it the Lie algebra of the group $\mathcal{G}$.

One can verify that $L \mathcal{R}=\mathcal{M}_{n} \cdot \theta_{n}$ and $L \mathcal{L}=\mathcal{M}_{p} \cdot \theta_{p}$ where $\theta_{n}=\theta_{\mathbb{R}^{n}}$ and $\theta_{p}=\theta_{\mathbb{R}^{p}}$ such that $1_{\mathbb{R}^{n}}$ and $1_{\mathbb{R}^{p}}$ are the identity maps of $\mathbb{R}^{n}$ and $\mathbb{R}^{p}$, respectively. Moreover, for any $f \in \mathcal{E}(n, p)$ one can define a $\mathcal{E}_{n}$-homomorphism

$$
t f: \theta_{n} \rightarrow \theta_{f}
$$

with $t f(\phi)=d f \circ \phi$. Also, the map

$$
\begin{aligned}
w f: \theta_{p} & \rightarrow \theta_{f} \\
\psi & \mapsto \psi \circ f
\end{aligned}
$$

is a $\mathcal{E}_{p}$-homomorphism and the module structure is induced via $f^{*}: \mathcal{E}_{p} \rightarrow \mathcal{E}_{n}$ with $f^{*}(\alpha)=\alpha \circ f$ for $\alpha \in \mathcal{E}_{p}$. The tangent spaces to the $\mathcal{G}$-orbits of $f$ at $f$ are given as follow:

$$
\begin{aligned}
& L \mathcal{R} . f=t f\left(\mathcal{M}_{n} . \theta_{n}\right), \\
& L \mathcal{L} . f=w f\left(\mathcal{M}_{p} \cdot \theta_{p}\right), \\
& L \mathcal{C} . f=f^{*}\left(\mathcal{M}_{p}\right) \cdot \theta_{f}, \\
& L \mathcal{A} . f=L \mathcal{R} . f+L \mathcal{L} . f, \\
& L \mathcal{K} . f=L \mathcal{R} . f+L \mathcal{C} . f .
\end{aligned}
$$

Remark 2.1. Note that the tangent space $\theta_{f}$ is a free $\mathcal{E}_{n}$-module of rank $p$. Therefore, if $\left(y_{1}, \ldots, y_{p}\right)$ is a local coordinate system in $\left(\mathbb{R}^{p}, 0\right)$, then the vector fields

$$
\left(\frac{\partial}{\partial y_{1}}\right) \circ f, \ldots,\left(\frac{\partial}{\partial y_{p}}\right) \circ f
$$

form a basis for $\theta_{f}$ along $f$. Thus, one can identify $\theta_{f}$ with $\mathcal{E}(n, p)$ and write the tangent spaces as follow

$$
\begin{aligned}
L \mathcal{R} . f & =\mathcal{M}_{n} \cdot\left\{\frac{\partial f}{\partial x_{1}}, \ldots, \frac{\partial f}{\partial x_{n}}\right\}, \\
L \mathcal{L} . f & =f^{*}\left(\mathcal{M}_{p}\right) \cdot\left\{e_{1}, \ldots, e_{p}\right\}, \\
L \mathcal{C} . f & =f^{*}\left(\mathcal{M}_{p}\right) . \mathcal{E}_{n} \cdot\left\{e_{1}, \ldots, e_{p}\right\},
\end{aligned}
$$

where $\left\{e_{1}, \ldots, e_{p}\right\}$ is the standard basis of $\mathbb{R}^{p}$ (considered as elements of $\mathcal{E}(n, p)$ ). 
Definition 2.2. For a given map-germ $f$ and a Mather group $\mathcal{G}$, the $\mathcal{G}$-codimension of $f$ is defined as $\operatorname{dim}_{\mathbb{R}}\left(\mathcal{M}_{n} . \mathcal{E}(n, p) / L \mathcal{G} . f\right)$ and is denoted by $\operatorname{cod}_{\mathcal{G}}(f)$. Also, the $\mathcal{G}_{e}$-codimension of $f$ is defined as

$$
\operatorname{cod}_{\mathcal{G}_{e}}(f)=\operatorname{dim}_{\mathbb{R}}\left(\mathcal{E}(n, p) / L_{e} \mathcal{G} \cdot f\right)
$$

where

$$
\begin{aligned}
& L_{e} \mathcal{R} . f=t f\left(\theta_{n}\right), \\
& L_{e} \mathcal{L} . f=w f\left(\theta_{p}\right), \\
& L_{e} \mathcal{C} . f=f^{*}\left(\mathcal{E}_{p}\right) \cdot f, \\
& L_{e} \mathcal{A} \cdot f=L_{e} \mathcal{R} . f+L_{e} \mathcal{L} . f, \\
& L_{e} \mathcal{K} . f=L_{e} \mathcal{R} . f+L_{e} \mathcal{C} . f .
\end{aligned}
$$

The space $L_{e} \mathcal{G} . f$ is so called the extended tangent space.

Note that, the diffeomorphisms in a Mather group keep the origin fixed (in the source and or in the target). When studying deformations, the singularity can move away from the origin, so the vector fields involved in defining the tangent spaces are allowed not to fix the origin. It is for this reason that, the extended tangent spaces are defined.

\subsection{Finite determinacy}

A germ $f$ is said to be $k$ - $\mathcal{G}$-determined if any $g$ with $j^{k} g=j^{k} f$ is $\mathcal{G}$ - equivalent to $f$. The $k$-jet of $f$ is then called a sufficient jet. The least integer $k$ with this property is called the degree of determinacy of $f$. A $\mathcal{G}$-determined germ is a $k$ - $\mathcal{G}$-determined germ for some integer $k$.

Theorem 2.2 (Theorem 1.2 in [33]). For each $f$ and $\mathcal{G}$, the following are equivalent:
(i) $f$ is $\mathcal{G}$-determined,
(ii) for some $k, \mathcal{M}_{n}^{k} \cdot \mathcal{E}(n, m) \subset L \mathcal{G} . f$,
(iii) $\operatorname{cod}_{\mathcal{A}}(f)<\infty$,
(iv) $\operatorname{cod}_{\mathcal{A}_{e}}(f)<\infty$.

\subsubsection{Versal Unfolding}

One of the most important concepts in the singularity theory of map germs is that of versal unfolding.

Definition 2.3. Let $f \in \mathcal{M}_{n} . \mathcal{E}(n, m)$. An a-parameter unfolding $(a, F)$ of $f$ is a map germ

$$
F:\left(\mathbb{R}^{n} \times \mathbb{R}^{a},(0,0)\right) \rightarrow\left(\mathbb{R}^{m} \times \mathbb{R}^{a},(0,0)\right)
$$

in the form $F(x, u)=(\bar{f}(x, u), u)$, where the map

$$
\bar{f}:\left(\mathbb{R}^{n} \times \mathbb{R}^{a},(0,0)\right) \rightarrow\left(\mathbb{R}^{m}, 0\right)
$$


satisfies $\bar{f}(x, 0)=f(x)$ and is called an a-parameter deformation of $f$.

It is important to clarify the following fact about the deformation $\bar{f}$ in Definition 2.3. Let $\bar{f}: W \times U \rightarrow V$ be a representative of the map-germ $\bar{f}$, where $W \times U$ is a neighbourhood of $(0,0) \in \mathbb{R}^{n} \times \mathbb{R}^{a}$ and $V$ is a neighbourhood of $0 \in \mathbb{R}^{m}$. For $u \in U$ denote by $\bar{f}_{u}: W \rightarrow V$ the smooth map given $\bar{f}_{u}(x)=\bar{f}(x, u)$. Then $\bar{f}_{0}(0)=0$ but $\bar{f}_{u}(0)$ is not necessarily the origin of $\mathbb{R}^{m}$ for $u \neq 0$. This means that the fibre $0 \times \mathbb{R}^{a}$ is not necessarily preserved by $F$. Also, the singularities of $\bar{f}_{u}$ may no longer be at the origin. This is why one needs to consider the extended groups $\mathcal{G}_{e}$.

Definition 2.4. Let $\mathcal{G}$ be a Mather group with the identity element $I_{\mathcal{G}}$.

(i) A morphism between two unfoldings $(a, F)$ and $(b, G)$ is a pair $(\alpha, \psi):(a, F) \rightarrow(b, G)$ with $\alpha:\left(\mathbb{R}^{a}, 0\right) \rightarrow(\mathcal{G}, I), \psi:\left(\mathbb{R}^{a}, 0\right) \rightarrow\left(\mathbb{R}^{b}, 0\right)$, such that

$$
\bar{f}_{u}=\alpha(u) \cdot \bar{g}_{\psi(u)}
$$

The unfolding $(a, F)$ is then said to be induced from $(b, G)$ by $(\alpha, \psi)$.

(ii) Two unfoldings $(a, F)$ and $(b, G)$ are $\mathcal{G}$-equivalent if there exists a morphism $(\alpha, \psi)$ : $(a, F) \rightarrow(b, G)$, where $\psi$ is invertible.

(iii) An unfolding $(a, F)$ of a map-germ $f$ is said to be $\mathcal{G}$-versal if any unfolding $(b, G)$ of $f$ can be induced from $(a, F)$.

An analogous definition can be given for the extended group $\mathcal{G}_{e}$ by substituting $\mathcal{G}$ with $\mathcal{G}_{e}$ in Definition 2.4 .

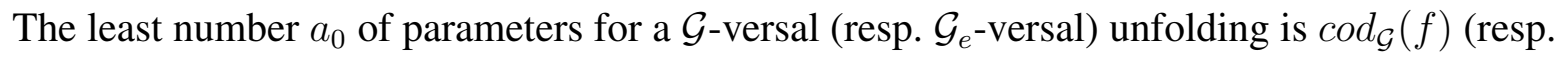
$\left.\operatorname{cod}_{\mathcal{G}_{e}}(f)\right)$ and the versal unfolding $\left(a_{0}, F\right)$ is called mini-versal. Mini-versal unfoldings are unique up to equivalence. Any versal unfolding is equivalent to a suspension of a mini-versal unfolding. Therefore, versal unfoldings $(a, F)$ and $(a, G)$ of $f$ are equivalent.

The importance of a $\mathcal{G}$-mini-versal unfolding (resp. $\mathcal{G}_{e}$-mini-versal unfolding) of a map-germ $f$ is that it provides a $\mathcal{G}$ (resp. $\mathcal{G}_{e}$ ) model of all possible local deformations of the map-germ $f$. (See [33] for more details).

Theorem 2.3 (Theorem 3.4 in [33]). For each map-germ $f$ and a Mather group $\mathcal{G}$, the following are equivalent:
(a) $f$ is $\mathcal{G}$-finite,
(b) $f$ has a $\mathcal{G}$-versal unfolding,
(c) f has a $\mathcal{G}_{e}$-versal unfolding. 
Given an unfolding $(a, F)$ of a map-germ $f$ in $\mathcal{M}_{n} . \mathcal{E}(n, m)$, denote by $\dot{F}_{i}, i=1, \ldots, a$, the map-germs in $\mathcal{M}_{n} \cdot \mathcal{E}(n, m)$ given by

$$
\dot{F}_{i}(x):=\frac{\partial \bar{f}}{\partial u_{i}}(x, 0),
$$

where $u_{i}$ 's are coordinates of the parameter space $\mathbb{R}^{a}$.

Theorem 2.4 (Theorem 3.3 in [33]). An unfolding $(a, F)$ of a map-germ $f$ in $\mathcal{M}_{n} \cdot \mathcal{E}(n, m)$ is $\mathcal{G}$-versal if and only if

$$
L \mathcal{G} . f+\mathbb{R} .\left\{\dot{F}_{1}, \ldots, \dot{F}_{a}\right\}=\mathcal{M}_{n} \cdot \mathcal{E}(n, m)
$$

and is $\mathcal{G}_{e^{-v e r s a l}}$ if and only if

$$
L \mathcal{G}_{e} . f+\mathbb{R} .\left\{\dot{F}_{1}, \ldots, \dot{F}_{a}\right\}=\mathcal{E}(n, m) .
$$

An unfolding $(a, F)$ of $f$ is said to be $\mathcal{G}$-trivial (resp. $\mathcal{G}_{e}$-trivial) if it is $\mathcal{G}$-equivalent (resp. $\mathcal{G}_{e}$-equivalent) to the constant unfolding $(a, f)$. A map-germ $f$ is $\mathcal{G}$-stable (resp. $\mathcal{G}_{e^{-}}$-stable) if all its unfoldings are trivial.

Theorem 2.5 (Theorem 3.8 in [24]). A map-germ $f$ is $\mathcal{G}$-stable (resp. $\mathcal{G}_{e^{-}}$-stable) if and only if $\operatorname{cod}_{\mathcal{G}}(f)=0\left(\right.$ resp. $\left.\operatorname{cod}_{\mathcal{G}_{e}}(f)=0\right)$.

\subsection{Germs of functions}

We deal here in some details with the case of germs of functions (i.e., $m=1$ ). The Hessian matrix of a germ of a function $f:\left(\mathbb{R}^{n}, 0\right) \rightarrow(\mathbb{R}, 0)$ is given by

$$
\mathcal{H}(f)(0)=\left(\frac{\partial^{2} f}{\partial x_{i} \partial x_{j}}\right) .
$$

If $f$ is singular at the origin, we say that this singularity is non-degenerate if $\operatorname{rank\mathcal {H}}(f)(0)=n$, equivalently, if and only if $\operatorname{det} \mathcal{H}(f)(0) \neq 0$.

Theorem 2.6 ([6]). Let $f:\left(\mathbb{R}, t_{0}\right) \rightarrow \mathbb{R}$ be smooth such that $f^{(p)}\left(t_{0}\right)=0$ for all $1 \leq p \leq k$, while $f^{(k+1)}\left(t_{0}\right) \neq 0$. Then $f \sim_{\mathcal{R}} g$, where $g:(\mathbb{R}, 0) \rightarrow \mathbb{R}$ is defined by $g(t)= \pm t^{k+1}$. We have + or - according to $f^{(k+1)}\left(t_{0}\right)$ is positive or negative.

Remark 2.2. (i) If $k$ is even, then $t^{k+1} \sim_{\mathcal{R}}-t^{k+1}$ where $h(t)=-t$. For $k$ odd they are not $\mathcal{R}$-equivalent.

(ii) For $k \neq l, t^{k+1}$ and $-t^{k+1}$ are not $\mathcal{R}$-equivalent to $t^{l+1}$ (both at $t=0$ ).

Definition 2.5. When $f:\left(\mathbb{R}, t_{0}\right) \rightarrow \mathbb{R}$ is $\mathcal{R}$-equivalent to $\pm t^{k+1}$, then we say $f$ has type $A_{k}$ at $t_{0}$ or has an $A_{k}$-singularity at $t_{0}$. 
An extensive list of $\mathcal{R}$-finite germs of functions is given in [3]. Table 2.1 shows the simple $\mathcal{R}$-finite germs of functions $\left(\mathbb{R}^{n}, 0\right) \rightarrow(\mathbb{R}, 0)$.

\begin{tabular}{ccc}
\hline Name & Normal forme & $\operatorname{cod}_{\mathcal{G}}(f)$ \\
\hline$A_{k}, k \geq 0$ & $\pm x_{1}^{k+1}+Q\left(x_{2}, \ldots, x_{n}\right)$ & $k$ \\
$D_{k}, k \geq 4$ & $x_{1}^{2} x_{2} \pm x_{2}^{k-1}+Q\left(x_{3}, \ldots, x_{n}\right)$ & $k$ \\
$E_{6}$ & $x_{1}^{3}+x_{2}^{4}+Q\left(x_{3}, \ldots, x_{n}\right)$ & 6 \\
$E_{7}$ & $x_{1}^{3}+x_{1} x_{2}^{4}+Q\left(x_{3}, \ldots, x_{n}\right)$ & 7 \\
$E_{8}$ & $x_{1}^{3}+x_{2}^{5}+Q\left(x_{3}, \ldots, x_{n}\right)$ & 8 \\
\hline$Q\left(x_{r}, \ldots, x_{n}\right)= \pm x_{r}^{2} \pm \cdots \pm x_{n}^{2}$. & & \\
\hline
\end{tabular}

Table 2.1 - Simple germs of functions ([3]).

Note that for the group $\mathcal{R}$, there are no $\mathcal{R}$-finitely determined map-germs in $\mathcal{M}_{n} \cdot \mathcal{E}(n, m)$ if $m>1$. Therefore, the group $\mathcal{R}$ is useful only when considering germs of functions.

As we will see in Chapter 3, it is important to consider also the direct product of the group $\mathcal{R}$ with translations, which we denote by $\mathcal{R}^{+}$.

Definition 2.6. Two families of germs of functions $F$ and $G:\left(\mathbb{R}^{n} \times \mathbb{R}^{a},(0,0)\right) \rightarrow(\mathbb{R}, 0)$ are $\mathcal{R}^{+}$-equivalent if there exist a germ of a diffeomorphism $\Phi:\left(\mathbb{R}^{n} \times \mathbb{R}^{a},(0,0)\right) \rightarrow\left(\mathbb{R}^{n} \times \mathbb{R}^{a},(0,0)\right)$ of the form $\Phi(x, u)=(\alpha(x, u), \psi(u))$ and a germ of a function $c:\left(\mathbb{R}^{a}, 0\right) \rightarrow \mathbb{R}$ such that

$$
G(x, u)=F(\Phi(x, u))+c(u) .
$$

Theorem 2.7 (Theorem 3.12 in [24]). A deformation $F:\left(\mathbb{R}^{n} \times \mathbb{R}^{a},(0,0)\right) \rightarrow(\mathbb{R}, 0)$ of a germ of a function $f$ in $\mathcal{M}_{n}$ is $\mathcal{R}^{+}$-versal if and only if

$$
L \mathcal{R}_{e} . f+\mathbb{R}\left\{\dot{F}_{1}, \ldots, \dot{F}_{n}\right\}=\mathcal{E}_{n}
$$

To verify $\mathcal{R}^{+}$-versality of an $r$-parameter family of a singularity of type $A_{k},(k \geq 1)$, we should prove that every real polynomial $p(t)$ of degree $\leq k-1$ and without constant term can be written in the form

$$
p(t)=\sum_{i=1}^{r} c_{i} j^{k-1}\left(\frac{\partial F}{\partial x_{i}}\left(t, x_{0}\right)(0)\right)
$$

for some real constants $c_{i}$, where $x_{i}$ denote the parameters for $i=1, \ldots, r$ see [6] for more details.

There is an equivalent formulation of this criterion. Let $j^{k-1}\left(\frac{\partial F}{\partial x_{i}}\left(t, x_{0}\right)(0)\right)=\alpha_{1 i} t+\alpha_{2 i} t^{2}+$ $\cdots+\alpha_{k-1, i} t^{k-1}$ for $k=1, \ldots, r$. Then $F$ is $\mathcal{R}^{+}$-versal if and only if the $r \times(k-1)$ matrix of coefficients $\left[\alpha_{i j}\right]$ has rank $k-1$ (see chapter 6 of [6]). 


\subsubsection{Discriminant and bifurcation sets}

To an $a$-parameter family of germs of functions $F$, we associate some germs of sets as follow. The catastrophe set $C_{F}$ of a family $F:\left(\mathbb{R}^{n} \times \mathbb{R}^{a},(0,0)\right) \rightarrow(\mathbb{R}, 0)$ is defined by

$$
C_{F}:=\left\{(x, u) \in\left(\mathbb{R}^{n} \times \mathbb{R}^{a},(0,0)\right) \mid \frac{\partial F}{\partial x_{1}}(x, u)=\cdots=\frac{\partial F}{\partial x_{n}}(x, u)=0\right\} .
$$

The bifurcation set of $F$ is defined by

$$
\operatorname{Bif}(F):=\left\{u \in\left(\mathbb{R}^{a}, 0\right) \mid \exists(x, u) \in C_{F} \text { and } \operatorname{rank}\left(\frac{\partial^{2} F}{\partial x_{i} \partial x_{j}}(x, u)\right)<n\right\} .
$$

The discriminant of $F$ is defined as

$$
\operatorname{Dis}(F)=\left\{u \in\left(\mathbb{R}^{a}, 0\right) \mid \exists x \in\left(\mathbb{R}^{n}, 0\right) \text { s.t. } F=\frac{\partial F}{\partial x_{1}}=\cdots=\frac{\partial F}{\partial x_{n}}=0 \text { at }(x, u)\right\} .
$$

Let $\pi_{C_{F}}=\pi_{\left.2\right|_{C_{F}}}: C_{F} \rightarrow\left(\mathbb{R}^{a}, 0\right)$, where $\pi_{2}:\left(\mathbb{R}^{n} \times \mathbb{R}^{a},(0,0)\right) \rightarrow\left(\mathbb{R}^{a}, 0\right)$ is the projection to the second component. We call $\pi_{C_{F}}$ the catastrophe map-germ of $F$.

Proposition 2.1. Let $F$ and $G$ be two families of germs of functions $\left(\mathbb{R}^{n} \times \mathbb{R}^{a}, 0\right) \rightarrow(\mathbb{R}, 0)$ such that their catastrophe sets $C_{F}$ and $C_{G}$ are smooth submanifolds. Suppose that $F$ and $G$ are $\mathcal{R}^{+}$-equivalent. Then the catastrophe map-germs $\pi_{C_{F}}$ and $\pi_{C_{G}}$ are $\mathcal{A}$-equivalent. Moreover, there exists a germ of a diffeomorphism $\phi:\left(\mathbb{R}^{n}, 0\right) \rightarrow\left(\mathbb{R}^{n}, 0\right)$ such that $\phi(\operatorname{Bif}(F))=\operatorname{Bif}(G)$.

Proof. See the Proposition 3.1 in [24].

Remark 2.3. Let $F:\left(\mathbb{R} \times \mathbb{R}^{k-1},(0,0)\right) \rightarrow(\mathbb{R}, 0)$ be an $\mathcal{R}^{+}$-versal family of an $A_{k}$-singularity. Then, the bifurcation set of $F$ is diffeomorphic to the discriminant of an $\mathcal{R}$-versal family of an $A_{k-1}$-singularity. Indeed, the family $F$ is given by

$$
F=b_{1} t+b_{2} t^{2}+\cdots+b_{k-1} t^{k-1}+t^{k+1} .
$$

Therefore Bif $(F)=\left\{u \in\left(\mathbb{R}^{k-1}, 0\right) \mid \exists t \in \mathbb{R} \quad\right.$ s.t. $\left.\quad \frac{\partial F}{\partial t}(t)=\frac{\partial^{2} F}{\partial t^{2}}(t)=0\right\}$. Consider the family $G:=\frac{\partial F}{\partial t}$, dividing the family $G$ by the coefficient of $t^{k}$, we have an $\mathcal{R}$-versal family of an $A_{k-1}$-singularity with its discriminant is diffeomorphic to bifurcation of $F$.

\subsection{Contact between submanifolds}

We can associate geometrical invariants to a submanifold $M \subset \mathbb{R}^{n}$ by comparing it with submanifold model in such a way that an invariant at a given point $p$ in $M$ is defined as that of the model that better approximates $M$ at $p$. We investigate in this section the concept of contact between submanifolds as a singularity theory tool for the study of differential geometry of submanifold of $\mathbb{R}^{n}$. 
Definition 2.7. Let $M_{i}, N_{i} \quad i=1,2$ submanifolds of $\mathbb{R}^{n}$ such that $\operatorname{dim}\left(M_{1}\right)=\operatorname{dim}\left(M_{2}\right)=m$, $\operatorname{dim}\left(N_{1}\right)=\operatorname{dim}\left(N_{2}\right)=d$. The contact of $M_{1}$ and $N_{1}$ at $y_{1}$ is the same type as the contact of $M_{2}$ and $N_{2}$ at $y_{2}$ if there exists a germ of diffeomorphism $\phi:\left(\mathbb{R}^{n}, y_{1}\right) \rightarrow\left(\mathbb{R}^{n}, y_{2}\right)$ such that $\phi\left(M_{1}\right)=M_{2}$ and $\phi\left(N_{1}\right)=N_{2}$.

The definition of contact between submanifolds in Definition 2.7 is local in nature, so the ambient space $\mathbb{R}^{n}$ can be replaced by any manifold $Z$.

Theorem 2.8 ([25]). Let $g_{i}:\left(M_{i}, x_{i}\right) \rightarrow\left(\mathbb{R}^{n}, 0\right)$ be immersion-germs and $f_{i}:\left(\mathbb{R}^{n}, 0\right) \rightarrow\left(\mathbb{R}^{k}, 0\right)$ submersion-germs, with $N_{i}=f_{i}^{-1}(0), i=1,2$. Then the pairs $\left(M_{1}, N_{1}\right)$ and $\left(M_{2}, N_{2}\right)$ have the same contact type if and only if $f_{1} \circ g_{1}$ and $f_{2} \circ g_{2}$ (as constant unfoldings) are $\mathcal{K}$-equivalent.

Proposition 2.2 ([22]). Let $M_{i}, i=1,2$, be two manifolds with $\operatorname{dim}\left(M_{1}\right)=\operatorname{dim}\left(M_{2}\right)=n-1$, and $g_{i}:\left(M_{i}, x_{i}\right) \rightarrow\left(\mathbb{R}^{n}, y_{i}\right)$ be germs of immersion. Let $f_{i}:\left(\mathbb{R}^{n}, y_{i}\right) \rightarrow(\mathbb{R}, 0)$ be germs of submersions and $F_{f}=\left\{f^{-1}(c) \mid c \in(\mathbb{R}, 0)\right\}$. Then the contact of $g_{1}\left(M_{1}\right)$ with $F_{f_{1}}$ at $y_{1}$ is of the same type as the contact of $g_{2}\left(M_{2}\right)$ with $F_{f_{2}}$ at $y_{2}$ if and only if $f_{1} \circ g_{1}$ and $f_{2} \circ g_{2}$ are $\mathcal{R}^{+}$-equivalent.

In this section we consider the contact of a manifold $M$ immersed in $\mathbb{R}^{n}$ with families of submanifolds and show that this contact is generic in the following sense.

The space of smooth maps between two manifolds $X$ and $Y$ denotes by $C^{\infty}(X, Y)$. This space is endowed with Whitney $C^{\infty}$-topology. (For more details one can see for example [24] ). Let $\operatorname{Imm}(X, Y)$ denote the subset of $C^{\infty}(X, Y)$ whose elements are proper $C^{\infty}$-immersions of $X$ to $Y$ and $\operatorname{Emb}(X, Y)$ the space of proper $C^{\infty}$-embeddings of $X$ into $Y$. The set $\operatorname{Imm}(X, Y)$ (resp. $\operatorname{Emb}(X, Y))$ is equipped with induced Whitney $C^{\infty}$-topology. With this topology, the set $\operatorname{Imm}(X, Y)$ (resp. $\operatorname{Emb}(X, Y))$ is an open subset of $C^{\infty}(X, Y)$ and when $\operatorname{dim}(Y) \geq \operatorname{dim}(X)$, $\operatorname{Imm}(X, Y)$ is also dense in $C^{\infty}(X, Y)$. When $\operatorname{dim}(Y) \geq 2 \operatorname{dim}(X)+1$, the set $\operatorname{Emb}(X, Y)$ is dense in $C^{\infty}(X, Y)$, and when $X$ is compact, it is also open.

A property $P$ in the topological space $\operatorname{Imm}(X, Y)(\operatorname{resp} . \operatorname{Emb}(X, Y))$ is said to be generic if it is satisfied by a residual subset of $\operatorname{Imm}(X, Y)(\operatorname{resp} . \operatorname{Emb}(X, Y))$. A residual subset of a topological space is a countable intersection of open dense subsets.

\subsubsection{The family of height functions}

A hyperplane in $\mathbb{R}^{n}$ is determined by a unit vector $w$ in $\mathbb{R}^{n}$ and a scalar $r$. If $H(w, r)$ denotes such a hyperplane, then

$$
H(w, r)=\left\{y \in \mathbb{R}^{n} \mid\langle y, w\rangle-r=0\right\},
$$

where $\langle$,$\rangle is the scalar product in \mathbb{R}^{n}$. We are interested in the contact of submanifolds with families of parallel hyperplanes. The family of height functions $\mathcal{H}: \mathbb{R}^{n} \times S^{n-1} \rightarrow \mathbb{R}$ is defined by

$$
\mathcal{H}(y, w)=\langle y, w\rangle .
$$


Given an immersion $g: M \rightarrow \mathbb{R}^{n}$ of a submanifold $M$ into $\mathbb{R}^{n}$, we consider the family of height functions $H: M \times S^{n-1} \rightarrow \mathbb{R}$ on $M$ defined by $H(p, w)=\mathcal{H}(g(p), w)=\langle g(p), w\rangle$.

For $w$ fixed, we denote by $h_{w}: M \rightarrow \mathbb{R}$ the function given by $h_{w}(p)=H(p, w)$. Following Theorem 2.8, the contact of $g(M)$ with the family of parallel hyperplanes determined by $w \in$ $S^{n-1}$ is measured by the $\mathcal{K}$-singularities of the function $h_{w}$.

Theorem 2.9 ([24]). (i) For an open and dense set of immersions of a smooth curve $C$ in $\mathbb{R}^{n}$, $n \geq 2$, the family $H$ is locally $\mathcal{R}^{+}$-versal.

(ii) For an open and dense set of immersions of a 2-dimensional surface $M$ in $\mathbb{R}^{n}$, with $3 \leq n \leq 7$, the family $H$ is locally $\mathcal{R}^{+}$-versal.

Theorem 2.10 ([24]). (i) For a generic immersed curve $C$ in $\mathbb{R}^{n}$, the local $\mathcal{K}$ - singularities of $h_{w}$ are of type $A_{k}, k=1, \ldots, n$.

(ii) For a generic immersed surface $M$ in $\mathbb{R}^{3}$, the local $\mathcal{K}$ - singularities of $h_{w}$ are of type $A_{k}$, $k=1,2,3$.

Example 2.1 (Contact of plane curves with lines). Let $\gamma$ be a plane curve and $\mathbf{w} \in S^{1}$ a unit vector. When $\gamma$ is a smooth curve the function $h_{w}$ is singular at $t_{0}$ if and only if $w= \pm \mathbf{n}\left(t_{0}\right)$. Then it has an $A_{1}$-singularity if and only if $\kappa\left(t_{0}\right) \neq 0$. The singularity is of type $A_{2}$ if and only if $\kappa\left(t_{0}\right)=0$ and $\kappa^{\prime}\left(t_{0}\right) \neq 0$, that is $t_{0}$ is a first order inflection of $\gamma$ (which we also call an ordinary inflection).

According to Theorem 2.10, for generic curves, the only local singularities of $h_{w}$ are those of type $A_{1}$ and $A_{2}$. In 1-parameter families of curves $\gamma_{s}$, we can get an $A_{3}$-singularity for isolated values of the parameter, say at $s=0$, and this happens when $\kappa_{0}\left(t_{0}\right)=\kappa_{0}^{\prime}\left(t_{0}\right)=0$ but $\kappa_{0}^{\prime \prime}\left(t_{0}\right) \neq 0$, that is $t_{0}$ is a second order inflections of $\gamma_{0}$. Note that for families of curves we denote the family of height functions by $\tilde{H}$.

\subsubsection{Family of distance squared functions}

A hypersphere in $\mathbb{R}^{n}$ is determined by its centre $a \in \mathbb{R}^{n}$ and its radius $r$. Let

$$
C(a, r)=\left\{y \in \mathbb{R}^{n} \mid\langle y-a, y-a\rangle-r^{2}=0\right\}
$$

We are interested in the contact of a submanifold with a family of hyperspheres with the same centre which is the family of distance squared functions $D: \mathbb{R}^{n} \times \mathbb{R}^{n} \rightarrow \mathbb{R}$ such that

$$
D(y, a)=\langle y-a, y-a\rangle .
$$

Given an immersion $g: M \rightarrow \mathbb{R}^{n}$ of a submanifold $M$ into $\mathbb{R}^{n}$, we consider the family of distance squared functions $D: M \times \mathbb{R}^{n} \rightarrow \mathbb{R}$ on $M$ defined by

$$
D(p, a)=D(g(p), a)=\langle g(p)-a, g(p)-a\rangle .
$$


For $a$ fixed, we denote by $D_{a}: M \rightarrow \mathbb{R}$ the function given by $D_{a}(p)=D(p, a)$. Following Proposition 2.2, the contact of $g(M)$ with the family of hyperspheres with the same centre $a \in \mathbb{R}^{n}$ is measured by the $\mathcal{R}^{+}$-singularities of the function $D_{a}$.

Theorem 2.11 ([24]). (i) For an open and dense set of immersions of a curve $C$ in $\mathbb{R}^{n}, n \geq 2$ the family $D$ is locally $\mathcal{R}^{+}$- versal.

(ii) For an open and dense set of immersions of a 2-dimensional surface $M$ in $\mathbb{R}^{n}$, with $3 \leq n \leq 6$, the family $D$ is locally $\mathcal{R}^{+}$-versal.

Theorem 2.12 ([24]). For a generic immersed curve $C$ in $\mathbb{R}^{n}$, the $\mathcal{K}$-singularities of $D_{a}$ are of type $A_{k}, k=1, \ldots, n+1$.

Theorem 2.13 ([24]). For a generic immersed surface $M$ in $\mathbb{R}^{3}$, the $\mathcal{K}$ - singularities of $D_{a}$ are of type $A_{k}, k=1,2,3,4$ or $D_{4}$.

Example 2.2 (Contact of plane curves with circles). When $\gamma$ is a smooth plane curve, we parametrise it by arc length and denote by $\mathbf{t}$ and $\mathbf{n}$ its unit tangent and normal vectors, respectively. The function $D_{a}, a \in \mathbb{R}^{2}$, is singular at $t_{0}$ if and only if $a=\gamma\left(t_{0}\right)+\lambda \mathbf{n}\left(t_{0}\right)$ for some scalar $\lambda$. If the curvature $\kappa\left(t_{0}\right) \neq 0$, the singularity of $D_{a}$ is of type $A_{2}$ if and only if $a=\gamma\left(t_{0}\right)+1 / \kappa\left(t_{0}\right) \mathbf{n}\left(t_{0}\right)$ and $\kappa^{\prime}\left(t_{0}\right) \neq 0$, that is $t_{0}$ is not a vertex of $\gamma$. Varying $t$ (and assuming that the curvature does not vanish in $t$ ) we get the evolute of the curve $\gamma$ parametrised by

$$
e(t)=\gamma(t)+\frac{1}{\kappa(t)} \mathbf{n}(t)
$$

The singularity of $D_{a}$ is of type $A_{3}$ if and only if $a$ is on the evolute and $\kappa^{\prime}\left(t_{0}\right)=0$ but $\kappa^{\prime \prime}\left(t_{0}\right) \neq 0$, that is $t_{0}$ is a first order vertex of $\gamma$ (which we also call an ordinary vertex). According to Theorem 2.12, for generic curves, the only local singularities of $D_{a}$ are $A_{1}, A_{2}, A_{3}$. The family $D$ is an $\mathcal{R}^{+}$-versal deformation of the generic singularities of its members (see Theorem 2.11). As the evolute is the bifurcation set of $D$, it follows that it is a smooth curve at an $A_{2}$-singularity of $D_{a}$ and a curve with a cusp singularity at an $A_{3}$-singularity of $D_{a}$; Figure 3.1. (See also [3] for an interpretation of the evolute as a caustic with generating family the family D.)

In 1-parameter families of curves $\gamma_{s}, D_{(a, s)}$ can have an $A_{4}$-singularity for isolated values of the parameter $s$, say at $s=0$, and this happens if $a$ is on the evolute of $\gamma_{0}$ and $\kappa_{0}^{\prime}\left(t_{0}\right)=\kappa_{0}^{\prime \prime}\left(t_{0}\right)=0$ but $\kappa_{0}^{\prime \prime \prime}\left(t_{0}\right) \neq 0$, that is $t_{0}$ is a second order vertex of $\gamma_{0}$. For generic families of curves (meaning that the big family of distance squared functions $\tilde{D}(t, a, s)=\left\langle\gamma_{s}(t)-a, \gamma_{s}(t)-a\right\rangle$ is an $\mathcal{R}^{+}$-versal deformation of the $A_{4}$-singularity of $D_{(a, 0)}$ on $\gamma_{0}$ ), the evolute of $\gamma_{s}$ undergoes the swallowtail transitions in Figure 3.2. 


\subsubsection{Family of projections into hyperplanes}

We consider here the contact of a submanifold in $\mathbb{R}^{n}$ with lines. We shall bundle together all parallel lines and represent them by their unit direction vectors $w \in S^{n-1}$. We choose $w^{\perp}$ to be the hyperplane orthogonal to $w$. This is precisely $T_{w} S^{n-1}$, the tangent space to the unit sphere $S^{n-1}$ at $w$. The family of orthogonal projections is $P: \mathbb{R}^{n} \times S^{n-1} \rightarrow T S^{n-1}$ given by

$$
P(y, w)=(w, y-\langle y, w\rangle w) .
$$

Given an immersion $g: M \rightarrow \mathbb{R}^{n}$ of a submanifold $M$ into $\mathbb{R}^{n}$, we consider the family of orthogonal projections $P: M \times S^{n-1} \rightarrow T S^{n-1}$ on $M$ defined by

$$
P(p, w)=P(g(p), w)=(w, g(p)-\langle g(p), w\rangle w) .
$$

Following Theorem 2.8, the contact of $g(M)$ with the family of parallel lines to $w \in S^{n-1}$ is measured by the $\mathcal{K}$-singularities the mapping $P_{w}$ given by

$$
P_{w}(p)=g(p)-\langle g(p), w\rangle w .
$$

Remark 2.4. The contact group $\mathcal{K}$ is a natural one to use when one seeks to understand the singularities of the zero level-sets of map-germs in $\mathcal{M}_{n} \mathcal{E}(n, m)$. If two germs are $\mathcal{K}$-equivalent, then their zero level-sets are diffeomorphic. The action of $\mathcal{A}$ is finer than that of $\mathcal{K}$. If two map-germs $F$ and $G$ are $\mathcal{A}$-equivalent, then $G=k \circ F \circ h^{-1}$ for some $(h, k) \in \mathcal{A}$. So the level sets $G^{-1}(c)$ and $F^{-1}\left(k^{-1}(c)\right)$ are diffeomorphic for any c close to $0 \in \mathbb{R}^{p}$. Therefore, $\mathcal{A}$ preserves the smooth structure of nearby level sets to the zero level set.

For surfaces in $\mathbb{R}^{3}$, given a point $p \in M$, we choose a local parametrisation $\mathbf{x}: U \subset \mathbb{R}^{2} \rightarrow M$ of $M$ at $p$ with $\mathbf{x}(0)=p$. The following Theorem gives information about contact of $M$ with lines.

Theorem 2.14 (Theorem 4.12 of [24]). For an open and dense set of immersions of a surface $M$ in $\mathbb{R}^{3}$, the family of orthogonal projections is locally $\mathcal{A}_{e^{-v}}$ ersal. The local $\mathcal{A}$-singularities of $P_{w}$ are those in Table 2.2.

\begin{tabular}{ccc}
\hline Name & Normal form & $\mathcal{A}_{e}$-codimension \\
\hline Immersion & $(x, y)$ & 0 \\
Fold & $\left(x, y^{2}\right)$ & 0 \\
Cusp & $\left(x, x y+y^{3}\right)$ & 0 \\
$4_{2}$ (Lips/Beaks) & $\left(x, y^{3} \pm x^{2} y\right)$ & 1 \\
$4_{3}$ (Goose) & $\left(x, y^{3} \pm x^{3} y\right)$ & 2 \\
5 (Swallowtail) & $\left(x, x y+y^{4}\right)$ & 1 \\
6 (Butterfly) & $\left(x, x y+y^{5} \pm y^{7}\right)$ & 2 \\
$11_{5}$ (Gulls) & $\left(x, x y^{2}+y^{4}+y^{5}\right)$ & 2 \\
\hline
\end{tabular}

Table 2.2 - Local singularities of projections of surfaces in $\mathbb{R}^{3}$ to planes. 


\subsection{Divergent diagrams}

In [14], Nuño-Ballesteros and Dias proposed a way to find models of the singularities of plane curves as well as their contact with lines at the singular point of the curve. They did this by classifying germs of divergent diagrams $(f, \alpha):(\mathbb{R}, 0) \stackrel{f}{\longleftarrow}(\mathbb{R}, 0) \stackrel{\alpha}{\longrightarrow}\left(\mathbb{R}^{2}, 0\right)$ up to smooth changes of coordinates. In the following we briefly explain the concept of $A_{h}$-equivalence defined in [14].

Definition 2.8. A divergent diagram $(f, \alpha):\left(\mathbb{R}^{p}, 0\right) \stackrel{f}{\leftarrow}\left(\mathbb{R}^{n}, 0\right) \stackrel{\alpha}{\rightarrow}\left(\mathbb{R}^{q}, 0\right)$ is a pair of smooth germs $\alpha:\left(\mathbb{R}^{n}, 0\right) \rightarrow\left(\mathbb{R}^{q}, 0\right)$ and $f:\left(\mathbb{R}^{n}, 0\right) \rightarrow\left(\mathbb{R}^{p}, 0\right)$.

Definition 2.9. Two divergent diagram $(f, \alpha)$ and $(g, \beta)$ are equivalent and we write $(f, \alpha) \sim$ $(g, \beta)$, if there exist germ of diffeomorphisms $H, K$ and $h$ such that the following diagrams commute:

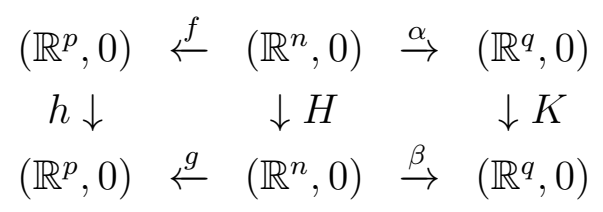

In particular for plane curves $\alpha$ and $\beta$, if $\left(h_{\alpha}, \alpha\right)$ and $\left(h_{\beta}, \beta\right)$ are equivalent, where $h_{\alpha}$ and $h_{\beta}$ are the height functions along the normal vectors of $\alpha$ and $\beta$ respectively, then $\alpha$ and $\beta$ are called $A_{h}$-equivalent and we denote this by $\alpha \sim_{A_{h}} \beta$.

For local singularities Dias and Nuño-Ballesteros classified all the plane curve diagrams $(f, \alpha)$, where $\alpha$ is a germ of plane curve with $A_{e}$-codimension $\leq 2$ and $f$ is any finitely determined germ of a function.

Proposition 2.3 ([14]). Let $(f, \alpha)$ be a plane curve diagram such that $\alpha$ is regular and $f$ has finite order $m$. Then $(f, \alpha)$ is equivalent to the diagram

$$
t^{m} \leftarrow t \rightarrow(t, 0)
$$

Assume now that $\alpha$ is singular. If $\alpha^{\prime \prime}(0) \neq 0$ and $\alpha$ is $\mathcal{A}$-finitly determined, then $\alpha$ is $\mathcal{A}$ equivalent to the $A_{2 k}$-singularity which is given by the map-germ $t \mapsto\left(t^{2}, t^{2 k+1}\right)$, with $k \geq 1$. If $k=1$, we have an ordinary cusp and the authors of [14] proved that $(f, \alpha)$ is equivalent to the diagram

$$
t^{m} \leftarrow t \rightarrow\left(t^{2}, t^{3}\right)
$$

For $k=2$ we have $A_{4}$-singularity $\left(t^{2}, t^{5}\right)$ which is called ramphoid cusp.

Proposition 2.4 ([14]). Let $(f, \alpha)$ be a plane curve diagram such that $\alpha$ is of type $A_{2 k}, k \geq 2$, and $f$ has finite order $m$. Then $(f, \alpha)$ is equivalent to either

$$
t^{m} \leftarrow t \rightarrow\left(t^{2}, t^{2 k+1}\right)
$$


or

$$
t^{m}+t^{m+2 r-1}+d_{1} t^{m+2 r+1}+\cdots+d_{k-r-1} t^{m+2 k-3} \leftarrow t \rightarrow\left(t^{2}, t^{2 k+1}\right)
$$

for some $1 \leq r \leq k-1$ and $d_{1}, \ldots, d_{k-r-1} \in \mathbb{R}$.

Corollary 2.1 ([14]). Let $(f, \alpha)$ be a plane curve diagram such that $\alpha$ is a ramphoid cusp and $f$ has finite order $m$. Then $(f, \alpha)$ is equivalent to either

$$
t^{m}+t^{m+1} \leftarrow t \rightarrow\left(t^{2}, t^{5}\right)
$$

or

$$
t^{m} \leftarrow t \rightarrow\left(t^{2}, t^{5}\right)
$$

Note that in [14] the authors have showed that two diagrams

$$
t^{m}+t^{m+1} \leftarrow t \rightarrow\left(t^{2}, t^{5}\right) \text { and } t^{m} \leftarrow t \rightarrow\left(t^{2}, t^{5}\right)
$$

are not equivalent. Moreover, if $\alpha(t)=\left(a_{2} t^{2}+a_{3} t^{3}+\ldots, b_{5} t^{5}+b_{6} t^{6}+\ldots\right)$, with $a_{2} b_{5} \neq 0$, and $f=c_{m} t^{m}+c_{m+1} t^{m+1}+\ldots$ with $c_{m} \neq 0$, then $(f, \alpha)$ is equivalent to

$$
\begin{cases}t^{m}+t^{m+1} \leftarrow t \rightarrow\left(t^{2}, t^{5}\right) & \text { if } 2 a_{2} c_{m+1}-m a_{3} c_{m} \neq 0, \\ t^{m} \leftarrow t \rightarrow\left(t^{2}, t^{5}\right) & \text { if } 2 a_{2} c_{m+1}-m a_{3} c_{m}=0 .\end{cases}
$$

Theorem 2.15 ([14]). Let $(f, \alpha)$ be a germ of plane curve diagram such that $\alpha$ has $\mathcal{A}_{e^{-}}$ codimension $\leq 2$ and $f$ has finite order $m$. Then $(f, \alpha)$ is equivalent to one of the diagrams in Table 2.3.

\begin{tabular}{lll} 
Type & & Germs \\
\hline$A_{0}$ & $t^{m}$ & $\leftarrow t \rightarrow(t, 0)$ \\
$A_{2}$ & $t^{m}$ & $\leftarrow t \rightarrow\left(t^{2}, t^{3}\right)$ \\
$A_{4}$ & $t^{m}+t^{m+1}$ & $\leftarrow t \rightarrow\left(t^{2}, t^{5}\right)$ \\
& $t^{m}$ & $\leftarrow t \rightarrow\left(t^{2}, t^{5}\right)$
\end{tabular}

Table 2.3 - Classification of divergent diagrams for plane curves with local singularities $A_{0}, A_{2}$ and $A_{4}$.

The above theorem is for general divergent diagrams. If the height function on the plane curve $\alpha$ is considered, then we have the concept of $A_{h}$-equivalence, as mentioned in the Definition 2.9. If two plane curves $\alpha$ and $\beta$ are $A_{h}$-equivalent, then they are $\mathcal{A}$-equivalent as well. But, the converse is not true.

The next theorem synthesize the $A_{h}$-classification of all the plane curve germs of $A_{e}$-codimension $\leq 2$.

Theorem 2.16 ([14]). Let $\alpha$ be a germ of plane curve singularity with $\mathcal{A}_{e}$-codimension $\leq 2$. Then $\alpha$ is $A_{h}$-equivalent to one of the local germs given in Table 2.4. 


\begin{tabular}{cc} 
Type & Germ \\
\hline$A_{0}$ & $\left(t, t^{k}\right), k \geq 2$ \\
$A_{2}$ & $\left(t^{2}, t^{3}\right)$ \\
$A_{4}$ & $\left(t^{2}, t^{4}+t^{5}\right)$ \\
& $\left(t^{2}, t^{5}+t^{6}\right)$ \\
& $\left(t^{2}, t^{5}\right)$ \\
\hline
\end{tabular}

Table 2.4 - Local $A_{h}$-classification of plane curves

Remark 2.5. For a given regular plane curve germ $\alpha$, if ord $\left(\kappa_{\alpha}\right)$ denotes the order of the curvature of $\alpha$, then ord $\left(\kappa_{\alpha}\right)$ is an $A_{h}$-invariant of $\alpha$. More precisely, $\alpha$ and $\beta$ are $A_{h}$-equivalent if and only if $\operatorname{ord}\left(\kappa_{\alpha}\right)=\operatorname{ord}\left(\kappa_{\beta}\right)$. Moreover, if $\kappa_{\alpha}(0)=\kappa_{\beta}(0)$ then $\alpha \sim_{A_{h}} \beta$, if and only if $\kappa_{\alpha} \sim_{\mathcal{A}} \kappa_{\beta}([14])$

\section{$2.7 \mathcal{K}(C)$-equivalence}

The problem of divergent diagram in $\$ 2.6$ is that the group acting is not a geometric subgroup. In [26] the authors introduced an alternative approach to that in [14]. They followed the method in [8] by fixing a model $X$ for the $\mathcal{A}$-singularity of the plane curve $\alpha$. Then the height function on $\alpha$ can be thought of as a submersion on $X$. They classified submersion up to smooth change of coordinates that preserve the model curve $X$. Here, the group acting is a geometric subgroup of the contact group $\mathcal{K}$, and the singularity theory classification techniques apply. In this section we summarize these concepts.

Suppose that $(X, 0) \subset\left(\mathbb{R}^{n}, 0\right)$ is the germ of reduced analytic subvariety of $\mathbb{R}^{n}$ at 0 defined by a polynomial $h$ in $\mathbb{R}\left[x_{1}, \ldots, x_{n}\right]$. A diffeomorphism $\phi:\left(\mathbb{R}^{n}, 0\right) \rightarrow\left(\mathbb{R}^{n}, 0\right)$ is said to preserve $X$ if $(\phi(X), 0)=(X, 0)$. The group of such diffeomorphisms is a subgroup of the group $\mathcal{R}$ which we denote by $\mathcal{R}(X)$. Also, $\mathcal{K}(X)$ denotes the subgroup of $\mathcal{K}$ given by $\mathcal{K}(X)=\mathcal{R}(X) \rtimes \mathcal{C}$.

Let $\Theta(X)$ be the $\mathcal{E}_{n}$-module of germs of vector fields in $\mathbb{R}^{n}$ tangent to $X$. The set of germs of vector fields in $\Theta(X)$ with no constant or linear terms is

$$
\Theta_{1}(X):=\left\{\zeta \in \Theta(X) \mid j^{1} \zeta=0\right\} .
$$

Definition 2.10. If $f$ is a smooth function, then $\Theta(X) . f=\mathcal{E}_{n} .\{\zeta f \mid \zeta \in \Theta(X)\}$ and we have the following tangent spaces to the $\mathcal{K}(X)$-orbits of $f$ at $f$.

$$
\begin{aligned}
L \mathcal{K}_{1}(X) \cdot f & =\Theta_{1}(X) \cdot f+f^{*}\left(\mathcal{M}_{1}^{2}\right) \cdot \mathcal{E}_{n} . \\
L \mathcal{K}(X) . f & =\Theta(X) \cdot f+f^{*}\left(\mathcal{M}_{1}\right) \cdot \mathcal{E}_{n} .
\end{aligned}
$$

Theorem 2.17 ([26]). Let $C$ be a plane curve with local singularity of $\mathcal{A}_{e}$-codimension $\leq 2$ i.e. $A_{0}, A_{2}$ and $A_{4}$-singularities. Then any $\mathcal{K}(C)$-finitely determined germ of a submersion $f:\left(\mathbb{R}^{2}, 0\right) \rightarrow(\mathbb{R}, 0)$ on a plane curve in column 2 of Table 2.5 is equivalent, by change of coordinates that preserve these curves, together with multiplication by germs of functions, to one of the germs in column 3 of Table 2.5. 


\begin{tabular}{cccc}
\hline Name & $\mathcal{A}$-model for $C$ & Submersion on $C$ & $\mathcal{K}(C)$-codim \\
\hline$A_{0}$ & $y$ & $y+x^{k}, k \geq 1$ & $k-2$ \\
\hline$A_{2}$ & $y^{2}-x^{3}$ & $x$ & 0 \\
& & $y$ & 1 \\
\hline$A_{4}$ & $y^{2}-x^{5}$ & $x$ & 0 \\
& & $y+x^{2}$ & 1 \\
& $y+x^{3}$ & 2 \\
& $y$ & 3 \\
\hline
\end{tabular}

Table 2.5 - Submersions on local singular plane curves.

In [26], the authors applied their results to the family of orthogonal projections of a generic space curve. For example, the bifurcation of generic projections of a space curve at a ramphoid cusp are as in Figure 2.1.

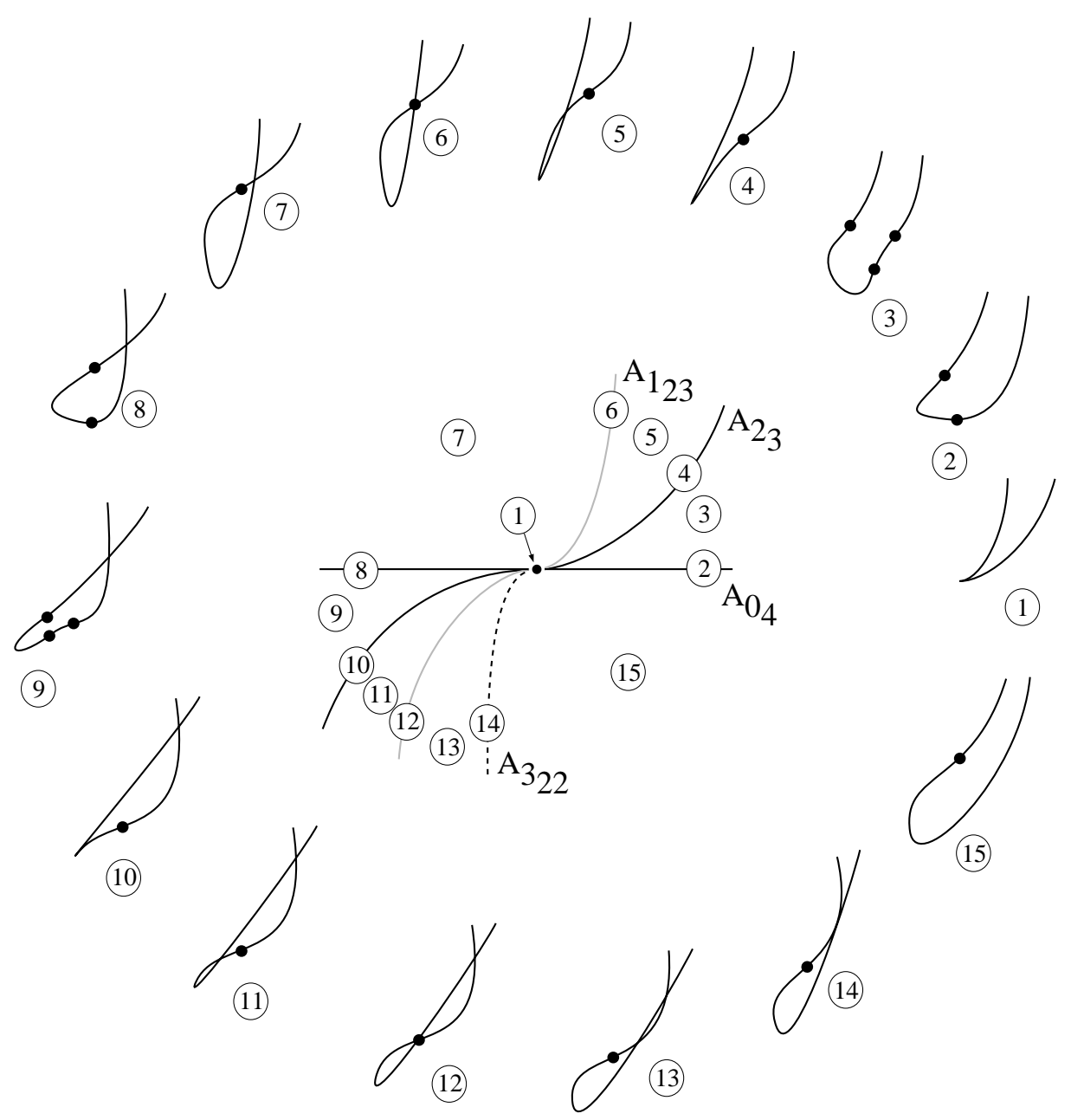

Figure 2.1 - Generic projections of space curves at a ramphoid cusp.

The $A_{0_{4}}$-stratum in Figure 2.1 occurs when the projected plane curve has a second order inflection. The $A_{2_{3}}$-stratum happens when $\alpha$ has cusp. The $A_{1_{23}}$-stratum is when a branch with 
an inflection intersects transversally another branch and $A_{322}$-stratum occurs when two branches become tangential (tacnode).

The flat geometry of the curve $\alpha$ is mainly about its inflections, which are obtained by considering the dual curve. The dual curve is the discriminant of the family of height functions on $\alpha$. The authors of [26] also considered the dual of the projection at a ramphoid cusp $\alpha$. 


\section{FLAT AND ROUND SINGULARITY THEORY OF PLANE CURVES}

We describe in this chapter a method for studying the geometry of deformations of singular plane curves, which we label $F R S$-deformation of plane curves ( $\mathrm{F}$ for flat, $\mathrm{R}$ for round and $\mathrm{S}$ for singular). When the curve is regular we label the deformations by $F R$-deformations.

We say that two germs of $m$-parameter deformations $\gamma_{s}$ and $\eta_{u}$ of a (singular) plane curve are $F R S$-equivalent if there exists a germ of a homeomorphism $k:\left(\mathbb{R}^{m},\left(S_{1}, 0\right)\right) \rightarrow\left(\mathbb{R}^{m},\left(S_{2}, 0\right)\right)$, where $S_{1}$ and $S_{2}$ are stratifications of the parameter spaces, such that

(i) $\gamma_{s}$ is diffeomorphic to $\eta_{k(s)}$ in each stratum of $S_{1}$;

(ii) $\gamma_{s}$ and $\eta_{k(s)}$ have the same number of inflections and vertices in each stratum;

(iii) the relative position of the singularities, points of self-intersections, inflections and vertices on $\gamma_{s}$ and $\eta_{k(s)}$ is the same in each stratum.

Of course we can define the notion of $F R S$-equivalence of deformations of a plane curve with distinct number of parameters. We say that an $m$-parameter deformation $\gamma_{s}$ and an $n$ parameter deformation $\eta_{u}$ (say $n \geq m$ ) of a plane curve are $F R S$-equivalent if $\tilde{\gamma}_{s}$ and $\eta_{u}$ are $F R S$-equivalent in the above sense, where $\tilde{\gamma}_{s}$ is the $n$-parameter family given by

$$
\tilde{\gamma}_{s}\left(t, s_{1}, \ldots, s_{m}, \tilde{s}_{m+1}, \ldots, \tilde{s}_{n}\right)=\gamma_{s}(t)
$$

We start with regular curves at inflections of any finite order ( 33.1$)$ and with the cusp $(\S 3.2)$ and ramphoid cusp (§3.3) singularities. We define in each case the meaning of a generic family and obtain the conditions for two such families to be $F R S$-equivalent. We also obtain the configuration of the deformed curve and of its evolute. 


\subsection{FR-deformations of regular curves}

Let $\gamma$ be a smooth and regular curve. Away from inflection points, the deformations of the curve at a vertex $t_{0}$ of finite order can be studied using the family of distance squared function $\tilde{D}(t, q, s)$. If the family $\tilde{D}$ on $\gamma_{s}$ with $\gamma_{0}=\gamma$ is an $\mathcal{R}^{+}$-versal deformation of the singularity of $\tilde{D}_{(q, s)}$ at $t_{0}$, then we have a well understood model of the deformation of the vertex of $\gamma$ and of the deformation of the evolute of $\gamma([2])$.

Suppose now that $\gamma$ has an inflection of finite order at $t_{0}$, i.e., $\kappa\left(t_{0}\right)=\kappa^{\prime}\left(t_{0}\right)=\ldots=$ $\kappa^{(k)}\left(t_{0}\right)=0, \kappa^{(k+1)}\left(t_{0}\right) \neq 0$ for some integer $k \geq 0$. To study the deformations of the inflection we use the family of height function $\tilde{H}$ on $\gamma_{s}$. If it is an $\mathcal{R}^{+}$-versal deformation, then we also have a well understood model of the deformation of the inflection. Observe that when $k \geq 1$, $\kappa^{\prime}\left(t_{0}\right)=0$ so we also have a vertex concentrated at the inflection point. The distance squared function at $t_{0}$ has an $A_{1}$-singularity and the evolute goes to infinity. To get information on the vertex of the curve and to understand the behavior of the evolute at infinity we compactify the plane (see also [34]). Consider the unit sphere $S^{2} \subset \mathbb{R}^{3}$ and the inverse of the stereographic projection $\phi: \mathbb{R}^{2} \rightarrow S^{2} \backslash\{(0,0,1)\}$ given by

$$
\phi(x, y)=\left(\frac{2 x}{1+x^{2}+y^{2}}, \frac{2 y}{1+x^{2}+y^{2}}, \frac{x^{2}+y^{2}-1}{1+x^{2}+y^{2}}\right) .
$$

The map $\phi$ takes circles in the plane to circles on the unit sphere. These are given by the intersection of $S^{2}$ with planes in $\mathbb{R}^{3}$. We can parametrise families of parallel planes by their common unit normal $\mathbf{v}=\left(v_{1}, v_{2}, v_{3}\right) \in S^{2}$ and we have a family of maps $\mathcal{D}: S^{2} \times S^{2} \rightarrow \mathbb{R}$ given by $\mathcal{D}(p, \mathbf{v})=\langle p, \mathbf{v}\rangle$. The fibres of $\mathcal{D}_{\mathbf{v}}(p)=\mathcal{D}(p, \mathbf{v})$ are the sought circles.

The contact of $\gamma$ with circles in $\mathbb{R}^{2}$ is the same as that $\phi(\gamma)$ with circles in $S^{2}$. Parametrise the family of curves in the form $\gamma_{s}(t)=\left(t, \beta_{s}(t)\right)$. Then the contact of $\phi\left(\gamma_{s}\right)$ with circles is measured by the singularities of the member of the family $\tilde{\mathcal{D}}: J \times S^{2} \times \mathbb{R}^{m} \rightarrow \mathbb{R}$ given by

$$
\tilde{\mathcal{D}}(t, \mathbf{v}, s)=\frac{2 v_{1} t+2 v_{2} \beta_{s}(t)+v_{3}\left(t^{2}+\beta_{s}(t)^{2}-1\right)}{1+t^{2}+\beta_{s}(t)^{2}} .
$$

Suppose that $\gamma=\gamma_{0}$ has an inflection at $t=0$. The function $\mathcal{D}_{(\mathbf{v}, 0)}$ is singular at $t=0$ if and only if $v_{1}=0$ and it has a degenerate singularity if and only if $v_{1}=v_{3}=0$, that is $\mathbf{v}=\mathbf{v}_{0}=(0,1,0)$. (Then the contact circle in the plane becomes a line at the inflection point and its image by $\phi$ is a great circle in $S^{2}$ through the north pole $(0,0,1)$.) The point $\mathbf{v}_{0}$ represent the point at infinity on the evolute of $\gamma$ at infinity. We have the following Proposition.

Proposition 3.1. The height function $\tilde{H}_{v_{0}}$ and the function $\tilde{\mathcal{D}}_{\left(v_{0}, 0\right)}$ have the same singularity type at $t=0$. Furthermore, the family $\tilde{\mathcal{D}}$ is an $\mathcal{R}^{+}$-versal deformation of the singularity of $\tilde{\mathcal{D}}_{\left(v_{0}, 0\right)}$ if and only if the family $\tilde{H}$ is an $\mathcal{R}^{+}$-versal deformation of the singularity of $\tilde{H}_{v_{0}}$.

Proof. According to Proposition 2.2, it is enough to prove that the height function $\tilde{H}_{\mathbf{v}_{0}}$ and the function $\tilde{\mathcal{D}}_{\left(\mathbf{v}_{0}, 0\right)}$ have the same $\mathcal{R}^{+}$-singularity type. Indeed, the height function $\tilde{H}_{\mathbf{v}_{0}}(t)=$ 
$\left\langle\gamma(t), v_{0}\right\rangle=\beta_{0}(t)$ has an $A_{\geq k}$-singularity at zero if and only if $\beta_{0}^{\prime}(0)=\cdots=\beta_{0}^{(k)}(0)=0$. On the other hand, considering the expression of $\mathcal{D}_{\left(\mathbf{v}_{0}, 0\right)}$ one can verify that $\mathcal{D}_{\left(\mathrm{v}_{0}, 0\right)}$ has an $A_{\geq k^{-}}$ singularity at zero if and only if $\beta_{0}^{\prime}(0)=\cdots=\beta_{0}^{(k)}(0)=0$. Let $\tilde{H}$ be an $(m+3)$-parameter family of an $A_{k}$-singularity. Therefore, we have

$$
\begin{aligned}
& j^{k-1}\left(\frac{\partial \tilde{H}}{\partial v_{1}}(t, 0)\right)(0)=t, \\
& j^{k-1}\left(\frac{\partial \tilde{H}}{\partial v_{2}}(t, 0)\right)(0)=j^{k-1} \beta_{0}(t), \\
& j^{k-1}\left(\frac{\partial \tilde{H}}{\partial s_{j}}(t, 0)\right)(0)=0 .
\end{aligned}
$$

Also, $\tilde{\mathcal{D}}$ is an $(m+3)$-parameter family of an $A_{k}$-singularity. Using the expression of $\tilde{\mathcal{D}}$ we have

$$
\begin{aligned}
& j^{k-1}\left(\frac{\partial \tilde{\mathcal{D}}}{\partial v_{1}}(t, 0)\right)(0)=j^{k-1}\left(\frac{2 t}{1+t^{2}+\beta_{0}(t)^{2}}\right), \\
& j^{k-1}\left(\frac{\partial \tilde{\mathcal{D}}}{\partial v_{2}}(t, 0)\right)(0)=j^{k-1}\left(\frac{2 \beta_{0}(t)}{1+t^{2}+\beta_{0}(t)^{2}}\right), \\
& j^{k-1}\left(\frac{\partial \tilde{\mathcal{D}}}{\partial s_{j}}(t, 0)\right)(0)=0 .
\end{aligned}
$$

Therefore, using Taylor expansion one can verify that the coefficient matrix of system (3.2) has rank $k-1$ if and only if the coefficient matrix of system (3.3) has rank $k-1$.

Remark 3.1. Another way to view Proposition 3.1 is as follows. When the family of height function is $\mathcal{R}^{+}$-versal, the resulting family of curvature functions $\kappa(t, s)$ is an $\mathcal{R}$-versal family (this gives the deformations of the inflections $\kappa(t, s)=0$ ). This in turn implies that the derivative $\frac{\partial \kappa}{\partial t}(t, s)$ is an $\mathcal{R}$-versal family (and we get the deformations of the vertices $\frac{\partial \kappa}{\partial t}(t, s)=0$ ).

Definition 3.1. As consequence of Proposition 3.1, we say that a deformation of a regular curve $\gamma$ at an inflection of finite order is FR-generic if the associated family of height functions is an $\mathcal{R}^{+}$-versal deformation of the singularity of the height function on $\gamma$ along its normal direction.

We consider now in details inflection points of order one, two and three which occur generically in 2-parameter families of curves. We start with some remarks on vertices of plane curves. Let $\gamma$ be parametrised by arc-length with unit tangent and normal vectors $\mathbf{t}$ and $\mathbf{n}$. Differentiating the parametrisation $e(t)=\gamma(t)+\frac{1}{\kappa(t)} \mathbf{n}(t)$ of the evolute we get $e^{\prime}(t)=-\frac{\kappa^{\prime}(t)}{\kappa(t)^{2}} \mathbf{n}(t)$, so the evolute is singular at a vertex $t_{0}$ of $\gamma$. At a vertex $t_{0}, e^{\prime \prime}\left(t_{0}\right)=-\frac{\kappa^{\prime \prime}\left(t_{0}\right)}{\kappa^{2}\left(t_{0}\right)} \mathbf{n}\left(t_{0}\right)$, and $e^{\prime \prime \prime}\left(t_{0}\right)=$ $\frac{2 \kappa^{\prime \prime}\left(t_{0}\right)}{\kappa\left(t_{0}\right)} \mathbf{t}\left(t_{0}\right)-\frac{\kappa^{\prime \prime \prime}\left(t_{0}\right)}{\kappa^{2}\left(t_{0}\right)} \mathbf{n}\left(t_{0}\right)$, so in the coordinate system with basis $\left\{\mathbf{t}\left(t_{0}\right), \mathbf{n}\left(t_{0}\right)\right\}$, we have

$$
j^{3}\left(e(t)-e\left(t_{0}\right)\right)=\left(\frac{2 \kappa^{\prime \prime}\left(t_{0}\right)}{\kappa\left(t_{0}\right)}\left(t-t_{0}\right)^{3},-\frac{\kappa^{\prime \prime}\left(t_{0}\right)}{\kappa^{2}\left(t_{0}\right)}\left(t-t_{0}\right)^{2}-\frac{\kappa^{\prime \prime \prime}\left(t_{0}\right)}{\kappa^{2}\left(t_{0}\right)}\left(t-t_{0}\right)^{3}\right) .
$$



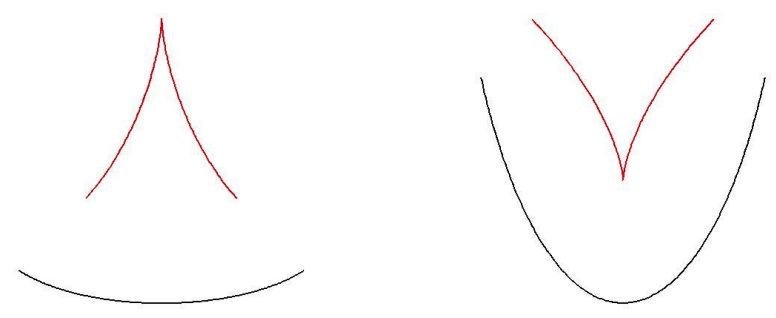

Figure 3.1 - Inward vertex (left) and outward vertex (right).
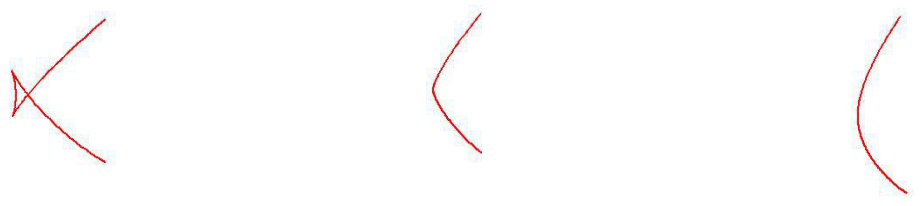

Figure 3.2 - Birth of an inward and an outward vertex in a generic deformation of a second order vertex.

It follows that at an ordinary vertex $\left(\kappa^{\prime \prime}\left(t_{0}\right) \neq 0\right)$, there are two possibilities for the cusp of the evolute: turning toward the curve or away from it (Figure 3.1). We have the configuration in Figure 3.1 left (resp. right) if $\kappa\left(t_{0}\right) \kappa^{\prime \prime}\left(t_{0}\right)>0$ (resp. $\left.\kappa\left(t_{0}\right) \kappa^{\prime \prime}\left(t_{0}\right)<0\right)$.

Definition 3.2. We call an ordinary vertex an inward vertex if the configuration of the curve and of its evolute at the vertex are as in Figure 3.1 left and an outward vertex if the configuration is as in Figure 3.1 right.

At a second order vertex, $\kappa^{\prime}\left(t_{0}\right)=\kappa^{\prime \prime}\left(t_{0}\right)=0$ and $\kappa^{\prime \prime \prime}\left(t_{0}\right) \neq 0$, the evolute has a singularity $\mathcal{A}$-equivalent to $\left(t^{3}, t^{4}\right)$. In a generic family of curves, the evolute undergoes the swallowtail transitions (Figure 3.2, [2, 5]). We have a birth of two vertices on the curve on one side of the transition, one is an inward vertex and the other is an outward vertex. (The curvature function does not change sign locally and it has one minimum at $t_{1}$ and one maximum at $t_{2}$, so $\kappa\left(t_{2}\right) \kappa^{\prime \prime}\left(t_{2}\right)$ and $\kappa\left(t_{2}\right) \kappa^{\prime \prime}\left(t_{2}\right)$ have different signs at the vertices $t_{1}$ and $t_{2}$.)

Proposition 3.2. If $\gamma$ has an ordinary inflection at $p$ then the evolute goes to infinity asymptotically along the normal line to $\gamma$ at $p$ and can be modeled in some coordinate system by $x y=1$. The two components of the evolute live in the two quadrants determined by the tangent and normal lines to $\gamma$ at $p$ which contain the curve $\gamma$ (see Figure 3.3).

Proof. At an ordinary inflection $\kappa=0$ and $\kappa^{\prime} \neq 0$. We parametrise the curve, without loss of generality, in the form $\gamma(t)=\left(t, b_{3} t^{3}+b_{4} t^{4}+O\left(t^{5}\right)\right)$ with $b_{3} \neq 0$ and $t$ varying in a small interval centred at zero. The ordinary inflection is at the origin and we have $\kappa(t)=$ 
$\frac{6 b_{3} t+12 b_{4} t^{2}+O\left(t^{3}\right)}{\left(1+\left(3 b_{3} t^{2}+4 b_{4} t^{3}+O\left(t^{4}\right)\right)^{2}\right)^{\frac{3}{2}}}$. The evolute of $\gamma$ is parametrised by

$$
\begin{aligned}
e(t)= & \left(t-\frac{1+\left(3 b_{3} t^{2}+4 b_{4} t^{3}+O\left(t^{4}\right)\right)^{2}}{6 b_{3} t+12 b_{4} t^{2}+O\left(t^{3}\right)}\left(3 b_{3} t^{2}+4 b_{4} t^{3}+O\left(t^{4}\right)\right),\right. \\
& \left.b_{3} t^{3}+b_{4} t^{4}+O\left(t^{5}\right)+\frac{1+\left(3 b_{3} t^{2}+4 b_{4} t^{3}+O\left(t^{4}\right)\right)^{2}}{6 b_{3} t+12 b_{4} t^{2}+O\left(t^{3}\right)}\right) \\
= & \left(\frac{1}{2} t+O\left(t^{2}\right), \frac{1}{6 b_{3} t}(1+O(t))\right.
\end{aligned}
$$

The above parametrisation gives, in particular, the position of the components of the evolute. If we put $x=\frac{1}{2} t+O\left(t^{2}\right)$ and $y=\frac{1}{6 b_{3} t}(1+O(t)$ then, using Implicit Function Theorem one can write $t=\phi_{1}(x)=x \phi_{2}(x)$ such that $\phi_{2}(0) \neq 0$. Substituting $t$ in $y$ we get $y=\frac{1}{3 b_{3} x \phi_{2}(x)}(1+O(x))$. Thus, $3 b_{3} x y \phi_{3}(x)=0$ such that $\phi_{3}(0) \neq 0$. Putting $X=3 b_{3} \phi_{3}(x)$ we obtain that the expression (3.4) can be written in the form $x y=1$ in this coordinate system.

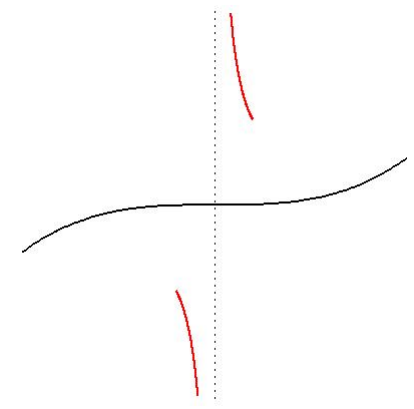

Figure 3.3 - Evolute of an ordinary inflection (normal line at the inflection in dots).

Second order inflections occur generically in 1-parameter families of curves. Following Proposition 3.1 and Remark 3.1, we take the family to be an $\mathcal{R}^{+}$-versal deformation of the $A_{3^{-}}$ singularity of the height function at the second order inflection. We express below the genericity of the family in terms of transversality to a stratification in the jet space.

A smooth curve $\gamma$ can be written at each point $p$ as the graph of a function $y=f_{p}(x)$, with $f_{p}(0)=f_{p}^{\prime}(0)=0$. Denote by $W^{k}$ the set of polynomials in one variable of $2 \leq$ degree $\leq k$. We have then the Monge-Taylor map $j^{k} \phi_{\gamma}: J \rightarrow W^{k}$, given by $j^{k} \phi_{\gamma}(t)=j^{k} f_{p}(0)$. One can identify the polynomial $j^{k} f_{p}(0)$ with its coefficients $\left(a_{2}, \ldots, a_{k}\right)$ so we can write $j^{k} \phi_{\gamma}(t)=\left(a_{2}, \ldots, a_{k}\right)$. A simple calculation shows that if $\gamma(t)=(t, \beta(t))$, then $\left(j^{k} \phi_{\gamma}\right)^{\prime}(0)=$ $\left(\frac{1}{2 !} \beta^{\prime \prime \prime}(0), \ldots, \frac{1}{k !} \beta^{(k+1)}(0)\right)$.

A curve $\gamma$ has an inflection at $t$ if and only if $j^{k} \phi_{\gamma}(t)$ belongs to the variety $I \subset W^{k}$ given by $a_{2}=0$. It has a vertex at $t$ if and only if $j^{k} \phi_{\gamma}(t)$ belongs to the variety $V \subset W^{k}$ given by $a_{3}=0$. The higher order inflections define a variety $H I \subset W^{k}$ which is given by $a_{2}=a_{3}=0$. Observe that $H I=I \cap V$, and at a second order inflection $\left(j^{k} \phi_{\gamma}\right)^{\prime}(0)$ is tangent to $I$ and transverse to $V$. A 1-parameter of curves $\gamma_{s}$, with $\gamma_{0}=\gamma$, induces a Monge-Taylor map $j^{k} \Phi:(\mathbb{R} \times \mathbb{R},(0,0)) \rightarrow W^{k}$ with $j^{k} \Phi(t, s)=j^{k} \phi_{\gamma_{s}}(t)$. A necessary condition for transversality of $\Phi$ to $H I$ is that the image of $d \Phi(0,0)$ is a plane. If that plane is transverse to $H I$, then it is transverse to $I$. We can always 
write $\gamma_{s}(t)=\left(t, \beta_{s}(t)\right)$. Then $d \Phi(0,0)$ is transverse to $H I$ if and only if $\frac{\partial^{3} \beta}{\partial s \partial t^{2}}(0,0) \neq 0$, which is exactly the condition for the family of height functions $\tilde{H}:\left(\mathbb{R} \times S^{1} \times \mathbb{R},\left(0, \mathbf{n}_{0}, 0\right)\right) \rightarrow \mathbb{R}$, with $\tilde{H}(t, \mathbf{v}, s)=\left\langle\gamma_{s}(t), \mathbf{v}\right\rangle$ to be an $\mathcal{R}^{+}$-versal deformation of the $A_{3}$-singularity of the height function on $\gamma$ along $\mathbf{n}_{0}=\mathbf{n}(0)$.

Theorem 3.1. Let $\gamma$ be a smooth and regular curve with a second order inflection point at $p$.

(i) The evolute goes to infinity asymptotically along the normal line to $\gamma$ at $p$ and can be modeled in some coordinate system by $x^{2} y=1$. The two components of the evolute live in the two quadrants determined by the tangent and normal lines to $\gamma$ at $p$ which contain the curve $\gamma$ (see Figure 3.4, center).

(ii) For an FR-generic family of curves $\gamma_{s}$ with $\gamma_{0}=\gamma$, the bifurcations in the evolute are as shown in Figure 3.4. We have a birth of two inflections and an appearance of one outward vertex on one side of the transition and of one inward vertex and no inflections on the other side of the transition.

(iii) Any FR-generic 1-parameter family of curves at a second order inflection is FRequivalent to the model family $\gamma_{u}(t)=\left(t, t^{4}+u t^{2}\right)$.

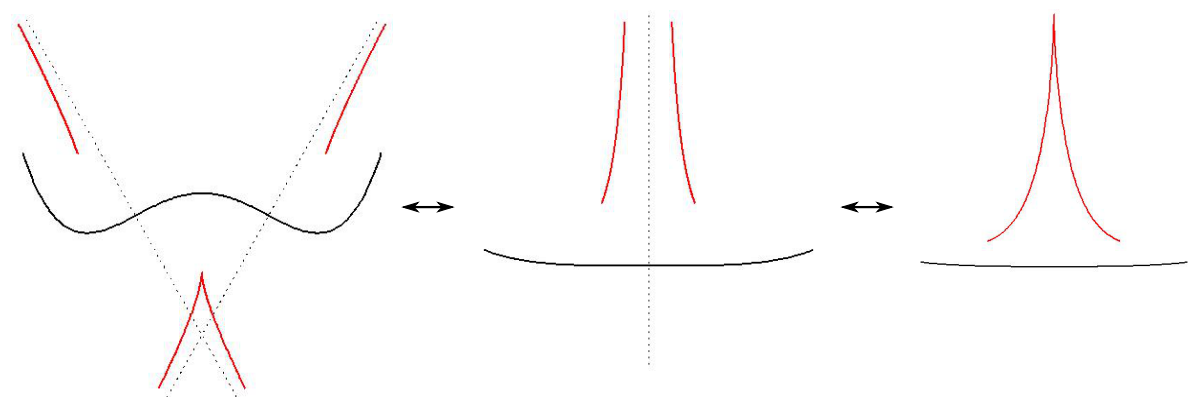

Figure 3.4-FR-Generic bifurcation of the evolute at a second order inflection of the curve (normal lines at the inflections in dots). The vertex changes from an outward to an inward vertex as it passes through the second order inflection.

Proof. At an inflection of second order we can parametrise $\gamma$ near $t=0$ in the form $\gamma(t)=$ $\left(t, b_{4} t^{4}+b_{5} t^{5}+O\left(t^{6}\right)\right)$ with $b_{4} \neq 0$. We have $\kappa(t)=\frac{12 b_{4} t^{2}+20 b_{5} t^{3}+O\left(t^{4}\right)}{\left(1+\left(4 b_{4} t^{3}+5 b_{5} t^{4}+O\left(t^{5}\right)\right)^{2}\right)^{\frac{3}{2}}}$, so the evolute of $\gamma$ is parametrised by $e(t)=\left(\frac{2}{3} t+O\left(t^{2}\right), \frac{1}{12 b_{4} t^{2}}(1+O(t))\right.$, and this gives (i).

For (ii), as the family is generic, we can change parameter if necessary and take $\gamma_{s}(t)=$ $\left(t, s t^{2}+\bar{b}_{3}(s) t^{3}+\bar{b}_{4}(s) t^{4}+O_{s}\left(t^{5}\right)\right)$, with $\bar{b}_{3}(0)=0, \bar{b}_{4}(0)=b_{4}$ and where $O_{s}\left(t^{5}\right)$ denotes a function in $(s, t)$ with the first four partial derivatives with respect to $t$ identically zero. The curvature function $\kappa_{s}(t)$ of $\gamma_{s}$ has an ordinary contact with the $t$-axis at $s=0$ (it has a maximum or a minimum at $t=0)$. Given the genericity condition, we have $\frac{\partial \kappa_{s}}{\partial s}(0,0) \neq 0$ so the graph $\kappa_{s}(t)$ intersects the $t$-axis in 2 points if $s<0$ and no points if $s>0$ or vice-versa. These points are (first order) inflection points of the curve. Of course the maximum or minimum of $\kappa_{s}$ is stable so 
we have one vertex on both sides of the transition and the vertex is between the two inflections when these are present. For the nature of the vertices, $\kappa_{s}^{\prime \prime}$ has locally constant sign and the sign of $\kappa_{s}$ at the vertex changes as $s$ crosses the value $s=0$. Thus $\kappa_{s} \kappa_{s}^{\prime \prime}$ has different sign at the vertices, so we have an inward vertex on one side of the transition (where there are no inflections) and an outward vertex on the other side of the transition (where there are two inflections). Combining this with (i) and Proposition 3.2, we can draw the bifurcations on the evolute in generic families and these are as in Figure 3.4 (Maple figure).

(iii) The calculations in (ii) depend only on the fact that the curve has a second order inflection and on the family being $F R$-generic. The family $\gamma_{u}(t)=\left(t, t^{4}+u t^{2}\right)$ satisfies these conditions, so it can be taken as an $F R$-model.

Theorem 3.2. Let $\gamma$ be a smooth and regular curve with a third order inflection point at $p$.

(i) The evolute goes to infinity asymptotically along the normal line to $\gamma$ at $p$ and can be modeled in some coordinate system by $x^{3} y=1$.

(ii) For an FR-generic 2-parameter family of curves $\gamma_{s}$ with $\gamma_{0}=\gamma$, the bifurcations in the evolute are as shown in Figure 3.5. The bifurcation diagram consists of a cusp curve corresponding to second order inflections and a smooth curve corresponding to second order vertices (see Figure 3.5, central figure).

(iii) Any FR-generic 2-parameter family of curves at a third order inflection is F R-equivalent to the model family $\gamma_{u}(t)=\left(t, t^{5}+u_{1} t^{3}+u_{2} t^{2}\right)$.

Proof. The proof is similar to that of Theorem 3.1. Here we can take an $F R$-generic 2-parameter family of curves in the form $\gamma_{s}(t)=\left(t, s_{1} t^{2}+s_{2} t^{3}+\bar{b}_{4}(s) t^{4}+\bar{b}_{5}(s) t^{5}+O_{s}\left(t^{6}\right)\right)$, with $\bar{b}_{4}(0)=0$ and $\bar{b}_{5}(0) \neq 0$ and $s=\left(s_{1}, s_{2}\right)$. Statement (ii) follows by analysing the curvature function $\kappa_{s}$. In fact, the curvature is given by

$$
\kappa_{s}(t)=\frac{O_{s}\left(t^{4}\right)+20 \bar{b}_{5} t^{3}+12 \bar{b}_{4}(s) t^{2}+6 s_{2} t+2 s_{1}}{\left(1+\left(O_{s}\left(t^{5}\right)+5 \bar{b}_{5}(s) t^{4}+4 \bar{b}_{4}(s) t^{3}+3 s_{2} t^{2}+2 s_{1} t\right)^{2}\right)^{3 / 2}}
$$

Then, calculating the first derivative of curvature and using Implicit Function Theorem, we find that the second order inflections stratum is given by $s=\left(-10 t^{2}+O\left(t^{3}\right), 20 t^{3}+O\left(t^{4}\right)\right)$ and the second order vertices stratum by $s_{2}=-\frac{2}{5} s_{1}^{6}+O\left(s_{1}^{7}\right)$. Statement (iii) follows from the fact that (ii) depends only on the family being $R$-generic.

\section{2 $\quad F R S$-deformations of a cusp}

Let $\gamma(t)=\gamma_{0}(t)=(\alpha(t), \beta(t)), t \in J$, and suppose that it is singular at $0 \in J$. For $t_{0} \in J$. If $\gamma$ is not infinitely flat at $t_{0}$, then it has a limiting normal line at $t_{0}$ and this line is part of the 
<smiles>CCC(C)(CC)CC</smiles>

(6)

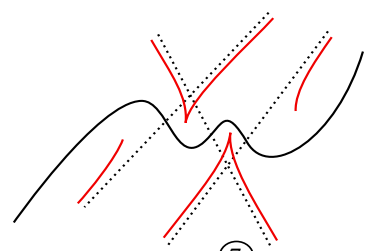

(7)

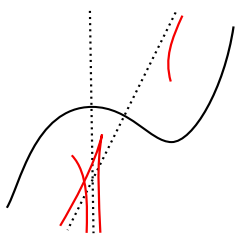<smiles>CCC(C)(CC)CC</smiles><smiles>CCC(CC)(CC)CC</smiles>

(1)

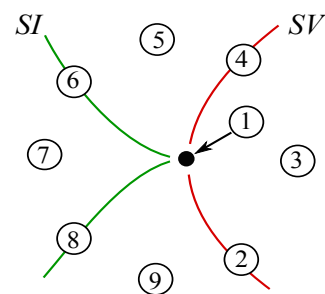

(9)

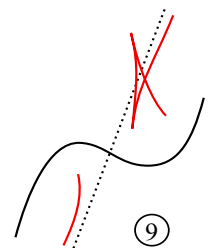

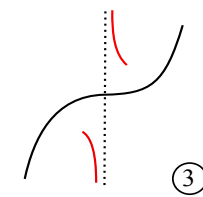

(3)
(9)
(2)

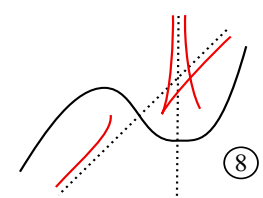

Figure 3.5 - FR-generic 2-parameter bifurcations of the evolute at a third order inflection (normal lines at the inflections in dots). The central figure is the bifurcation set and the stratum $S I$ (resp. $S V$ ) is of second order inflections (resp. vertices).

bifurcation set of the family of distance squared functions on the curve. We have the following observation.

Proposition 3.3. Let $\gamma_{s}$ be any m-parameter family of curves with $\gamma_{0}(t)=\gamma(t)$ singular at $t=0$. Then the big family of distance squared functions $\tilde{D}:\left(\mathbb{R} \times \mathbb{R}^{m} \times \mathbb{R}^{2},(0,0,0)\right) \rightarrow(\mathbb{R}, 0)$ on $\gamma$ with $\tilde{D}(t, a, s)=\left\langle\gamma_{s}(t)-a, \gamma_{s}(t)-a\right\rangle$ is never an $\mathcal{R}^{+}$-versal deformation of the singularity of $D_{0}(t)=\tilde{D}(t, 0,0)$ at $t=0$.

Proof. If $\gamma_{0}$ is infinitely flat, then it does not have an $\mathcal{R}^{+}$-versal deformation. Suppose now that it is not, and that the first non-zero derivative of $\gamma$ at $t=0$ is that of order $k$, with $k \geq 2$. We can then write the family of curves in the form $\gamma_{s}(t)=\left(\alpha_{s}(t), \beta_{s}(t)\right)$ with $\gamma_{0}=\gamma, \alpha_{0}(t)=t^{k}$ and $j^{k} \beta_{0}(t) \equiv 0$. Setting $a=\left(a_{1}, a_{2}\right)$, we have $\tilde{D}\left(t, a_{1}, a_{2}, s\right)=\left(\alpha_{s}(t)-a_{1}\right)^{2}+\left(\beta_{s}(t)-a_{2}\right)^{2}$. The 
function $D_{0}(t)=t^{2 k}+O\left(t^{2 k+2}\right)$ has an $A_{2 k-1}$-singularity at $t=0$ and $\left.j^{2} \frac{\partial \tilde{D}}{\partial a_{1}}\right|_{s=0, a=0}(t)=-2 t^{2}$, if $k=2$ or 0 if $k>2,\left.j^{2} \frac{\partial \tilde{D}}{\partial a_{2}}\right|_{s=0, a=0}(t)=0$, and $\left.j^{2} \frac{\partial \tilde{D}}{\partial s_{i}}\right|_{s=0, a=0}(t)=0, i=1, \ldots, m$. Therefore, $\tilde{D}$ can never be an $\mathcal{R}^{+}$-versal family of the $A_{2 k-1}$-singularity of $D_{0}$.

We can take $\gamma\left(t_{0}\right)$ to be the origin and write

$$
\begin{aligned}
\gamma(t)= & \left(\alpha^{\prime}\left(t_{0}\right)\left(t-t_{0}\right)+\frac{1}{2 !} \alpha^{\prime \prime}\left(t_{0}\right)\left(t-t_{0}\right)^{2}+\frac{1}{3 !} \alpha^{\prime \prime \prime}\left(t_{0}\right)\left(t-t_{0}\right)^{3}+O\left(\left(t-t_{0}\right)^{4}\right),\right. \\
& \left.\beta^{\prime}\left(t_{0}\right)\left(t-t_{0}\right)+\frac{1}{2 !} \beta^{\prime \prime}\left(t_{0}\right)\left(t-t_{0}\right)^{2}+\frac{1}{3 !} \beta^{\prime \prime \prime}\left(t_{0}\right)\left(t-t_{0}\right)^{3}+O\left(\left(t-t_{0}\right)^{4}\right)\right) .
\end{aligned}
$$

This gives the Taylor expansion map $j^{k} \phi_{\gamma}: J \rightarrow J^{k}(1,2)$, with

$$
j^{k} \phi_{\gamma}\left(t_{0}\right)=\left(\alpha^{\prime}\left(t_{0}\right), \frac{1}{2 !} \alpha^{\prime \prime}\left(t_{0}\right), \frac{1}{3 !} \alpha^{\prime \prime \prime}\left(t_{0}\right), \ldots ; \beta^{\prime}\left(t_{0}\right), \frac{1}{2 !} \beta^{\prime \prime}\left(t_{0}\right), \frac{1}{3 !} \beta^{\prime \prime \prime}\left(t_{0}\right), \ldots\right) .
$$

Denote by $\left(a_{1}, a_{2}, \ldots ; b_{1}, b_{2}, \ldots\right)$ the coordinate in $J^{k}(1,2)$ (identified with $\left.\mathbb{R}^{k} \times \mathbb{R}^{k}\right)$. We can stratify $J^{k}(1,2)$ into the following strata (together with the compliment of their union):

$$
\begin{aligned}
\text { Cusps }(C): & a_{1}=b_{1}=0, \\
\text { Inflections }(I): & a_{1} b_{2}-a_{2} b_{1}=0, \\
\text { Vertices }(V): & -2\left(a_{1} b_{2}-a_{2} b_{1}\right)\left(a_{1} a_{2}+b_{1} b_{2}\right)+\left(a_{1}^{2}+b_{1}^{2}\right)\left(a_{1} b_{3}-a_{3} b_{1}\right)=0 .
\end{aligned}
$$

For the inflections and vertices strata, we took only the numerators and ignored the denominators in the equations of $\kappa=0$ and $\kappa^{\prime}=0$. These vanish only at the singular points of the curve. There are of course additional $\neq 0$ (open) conditions in $C, I, V$ which are not included above.

At a point on the cusp stratum we have $a_{2} \neq 0$ or $b_{2} \neq 0$. At such points, the strata $I$ and $V$ are codimension 1 varieties in $J^{k}(1,2)$ which contain the codimension 2 variety $C$. The variety $I$ is smooth along $C$ and the variety $V$ is diffeomorphic to the union of 2 smooth varieties $V_{1}$ and $V_{2}$ intersecting transversally along $C$. Because note that we have $a_{2} b_{3}-a_{3} b_{2} \neq 0$ this implies that $a_{2}^{2}+b_{2}^{2} \neq 0$. Set $A_{1}=a_{1} b_{2}-a_{2} b_{1}$ and $B_{1}=a_{1} a_{2}+b_{1} b_{2}$ therefore

$$
\left(\begin{array}{l}
A_{1} \\
B_{1}
\end{array}\right)=\left(\begin{array}{cc}
b_{2} & -a_{2} \\
a_{2} & b_{2}
\end{array}\right)\left(\begin{array}{l}
a_{1} \\
b_{1}
\end{array}\right)
$$

and this implies that

$$
\left(\begin{array}{l}
a_{1} \\
b_{1}
\end{array}\right)=\frac{1}{a_{2}^{2}+b_{2}^{2}}\left(\begin{array}{cc}
b_{2} & a_{2} \\
-a_{2} & b_{2}
\end{array}\right)\left(\begin{array}{l}
A_{1} \\
B_{1}
\end{array}\right) .
$$

Applying the $a_{1}$ and $b_{1}$ in the equation of vertex stratum $V$ we get

$-2 A_{1} B_{1}+\left[\left(b_{2} A_{1}+a_{2} B_{1}\right)^{2}+\left(-a_{2} A_{1}+b_{2} B_{1}\right)^{2}\right]\left[\left(b_{2} A_{1}+a_{2} B_{1}\right) b_{3}-\left(-a_{2} A_{1}+b_{2} B_{1}\right) a_{3}\right]=0$.

Now set $A_{1}=\bar{A}_{1}+\bar{B}_{1}$ and $B_{1}=\bar{A}_{1}-\bar{B}_{1}$ one can get the vertex stratum diffeomorphic to the union of 2 smooth varieties.

The tangent cone of $V_{1}$ along $C$ is $a_{1} b_{2}-a_{2} b_{1}=0$ and that of $V_{2}$ is $a_{1} a_{2}+b_{1} b_{2}=0$, so $V_{1}$ is tangent to $I$ along $C$ and $V_{2}$ is transverse to it. In fact, we have a product stratification of $J^{k}(1,2)$ by $C, I, V$ along $C$ with a transverse slice given by its intersection with a well chosen plane. 
To simplify the calculations, we reparametrise $\gamma$, make isometric changes of coordinates in $\mathbb{R}^{2}$ and write

$$
\gamma(t)=\left(t^{2}, \beta(t)\right)=\left(t^{2}, b_{3} t^{3}+O\left(t^{4}\right)\right)
$$

with $b_{3} \neq 0$. In fact, any family of curves containing $\gamma=\gamma_{0}$ can be parametrised in the form

$$
\gamma_{s}(t)=\left(t^{2}, \beta(t, s)\right)=\left(t^{2}, \bar{b}_{1}(s) t+\bar{b}_{2}(s) t^{2}+\bar{b}_{3}(s) t^{3}+O_{s}\left(t^{4}\right)\right)
$$

A 1-parameter family of curves $\gamma_{s}$, with $\gamma_{0}=\gamma$, induces a Taylor expansion map $j^{k} \Phi$ : $(\mathbb{R} \times \mathbb{R},(0,0)) \rightarrow J^{k}(1,2)$ with $j^{k} \Phi(t, s)=j^{k} \phi_{\gamma_{s}}(t)$. If the image of $d\left(j^{k} \Phi\right)(0,0)$ is transverse to $C$, then it will be transverse to $I$ and $V$. If we take $\gamma_{s}$ as (3.6) then the transversality of $j^{k} \Phi$ to $C$ occurs if and only if $\frac{\partial^{2} \beta}{\partial s \partial t}(0,0) \neq 0$, which is the condition for the family of maps $\gamma_{s}$ to be an

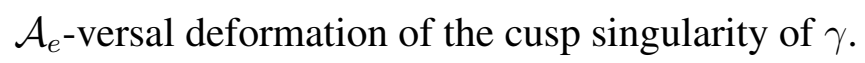

Definition 3.3. A germ of a 1-parameter family of curves $\gamma_{s}$ with $\gamma_{0}=\gamma$ a germ of a cusp curve, is said to be FRS-generic if it is an $\mathcal{A}_{e}$-versal deformation of the cusp singularity of $\gamma$.
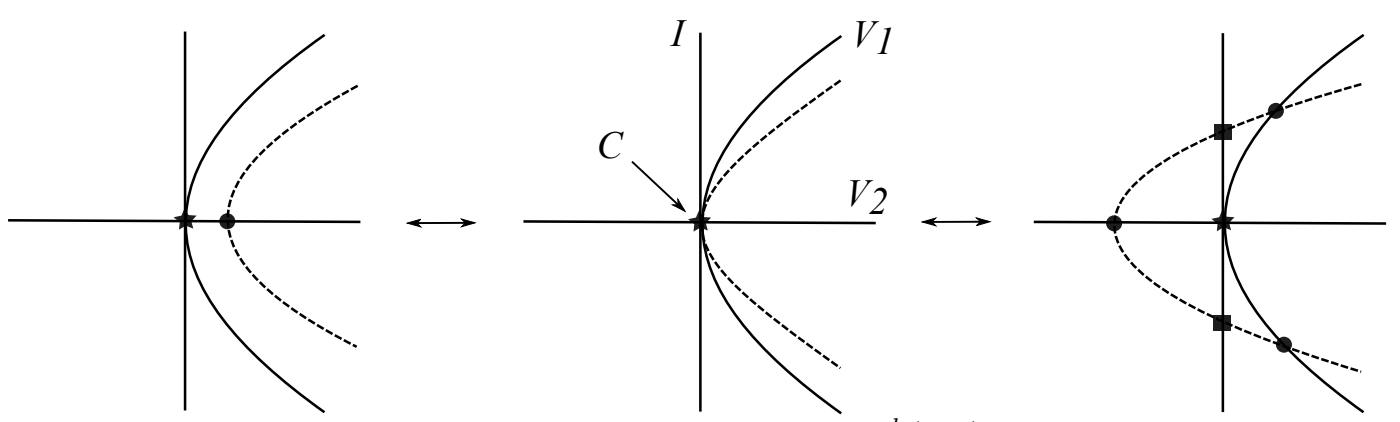

Figure 3.6 - A 2-dimensional transverse slice of the stratification of $J^{k}(1,2)$ by $C, I, V$. The figure in the centre shows the position of the image of the curve $\gamma_{0}$ by the Taylor expansion map (dashed) with respect to the stratification, and the left and right figures show the position of the image of the curve $\gamma_{s}, s<0$ left and $s>0$ right (or vice-versa) as well as its intersection with the inflections and vertices strata.

Theorem 3.3. Any FRS-generic 1-parameter family of a germ of the cusp curve is FRSequivalent to the FRS-model family $\left(t^{2}, t^{3}+u t\right)$.

Proof. We know that for an FRS-generic family of curves $\gamma_{s}$, the singularity of the curve is deformed as in Figure 3.7. In particular, we have a birth of two inflections on one side of the transition and none on the other. The inflections occur on the side where the curve has no self-intersection (see for example [26]). We have

$$
\left(j^{k} \phi_{\gamma_{0}}\right)^{\prime}\left(t_{0}\right)=\left(\alpha^{\prime \prime}\left(t_{0}\right), \frac{1}{2 !} \alpha^{\prime \prime \prime}\left(t_{0}\right), \frac{1}{3 !} \alpha^{(4)}\left(t_{0}\right), \ldots ; \beta^{\prime \prime}\left(t_{0}\right), \frac{1}{2 !} \beta^{\prime \prime \prime}\left(t_{0}\right), \frac{1}{3 !} \beta^{(4)}\left(t_{0}\right), \ldots\right),
$$

so for $\gamma_{0}$ parametrised as in (3.5) we have $\left(j^{k} \phi_{\gamma_{0}}\right)^{\prime}(0)=\left(2,0, \ldots, 0 ; 0,3 b_{3}, 0, \ldots, 0\right)$. This vector is clearly tangent to $I$ and $V_{1}$ and is transverse to $V_{2}$. The relative position of the image of $j^{k} \phi_{\gamma_{0}}$ and of the strata $C, I, V$ is as in Figure 3.6 center. As $\gamma_{s}$ is a generic family, the intersection 
of the image of $j^{k} \phi_{\gamma_{s}}$ with the strata $C, I, V$ is as in Figure 3.6, so we get, in particular, the position of the vertices and inflections on $\gamma_{s}$ (Figure 3.7, right). When there is one vertex (and no inflections), $j^{k} \phi_{\gamma_{s}}$ does not give the position of the vertex on $\gamma_{s}$ with respect to the point of selfintersection. Here we take the family $\gamma_{s}$ as in (3.6). As it is $F R S$-generic, we can take $\bar{b}_{1}(s)=s$. The vertex occurs at a point $t_{0}=O\left(s^{2}\right)$ and the points giving the point of self-intersection are $t= \pm t_{1}$ with $t_{1}^{2}=-\frac{1}{b_{3}} s+O\left(s^{2}\right)$. Clearly, for $s$ small enough, $-t_{1}<t_{0}<t_{1}$. This property depends only on the fact that the family $\gamma_{s}$ is generic and not on the family itself.

Any two generic families $\gamma(t, s)=\gamma_{s}(t)$ and $\eta(t, u)=\eta_{u}(t)$ of the cusp are $\mathcal{A}$-equivalent, so $\gamma(t, s)=\eta(h(t, s), k(s))$, with $h(t, 0)$ and $k(s)$ germs of a diffeomorphisms. The configuration in Figure 3.6 depends only on the fact that the family is $F R S$-generic, so the diffeomorphism $k$ satisfies the properties (i), (ii), (iii) in the definition of $F R S$-equivalence.

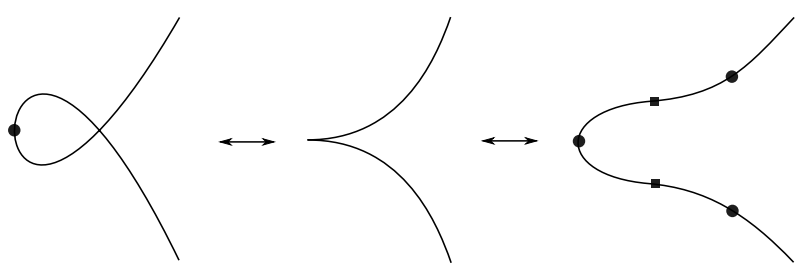

Figure 3.7 - Generic bifurcations of a cusp. Vertices (resp. inflections) are represented by circular (resp. square) dots.

Proposition 3.4. All the vertices that appear in an FRS-generic family of a cusp are outward vertices.

Proof. By direct computations we get $\kappa(t)=0$ at two points $t_{1}$ and $t_{2}$. Also, $\kappa^{\prime}(t)$ has 3 roots which means that the graph of $\kappa$ has 3 extrema $\bar{t}_{1}, \bar{t}_{2}$ and $\bar{t}_{3}$. Now straightforward calculations give the sign of $\kappa^{\prime \prime}(t) \kappa(t)$ in each region. See Figure 3.8 for more details.

We consider now the bifurcations in the evolute of $\gamma$. The distance squared function on a singular curve from any point on the normal line at the singular point has an $A_{\geq 2}$-singularity at the singular point of the curve. Thus, this normal line should be considered as part of the evolute. We call the closure of the centres of osculating circles of the curve at its regular points the proper evolute of the curve. We define the full evolute as the bifurcation set of the family of distance squared functions on the curve. The full evolute is the union of the proper evolute together with the normal line at the singular point counted with multiplicity.

Theorem 3.4. Let $\gamma$ be a plane curve with an ordinary cusp at $p$.

(i) ([19]) The proper evolute of $\gamma$ is a smooth curve and has an ordinary tangency at $p$ with the limiting normal line to $\gamma$ at $p$. The limiting normal line separates the curve and its evolute.

(ii) The full evolute of $\gamma$ consists of its proper evolute together with its limiting normal line at p counted twice. 


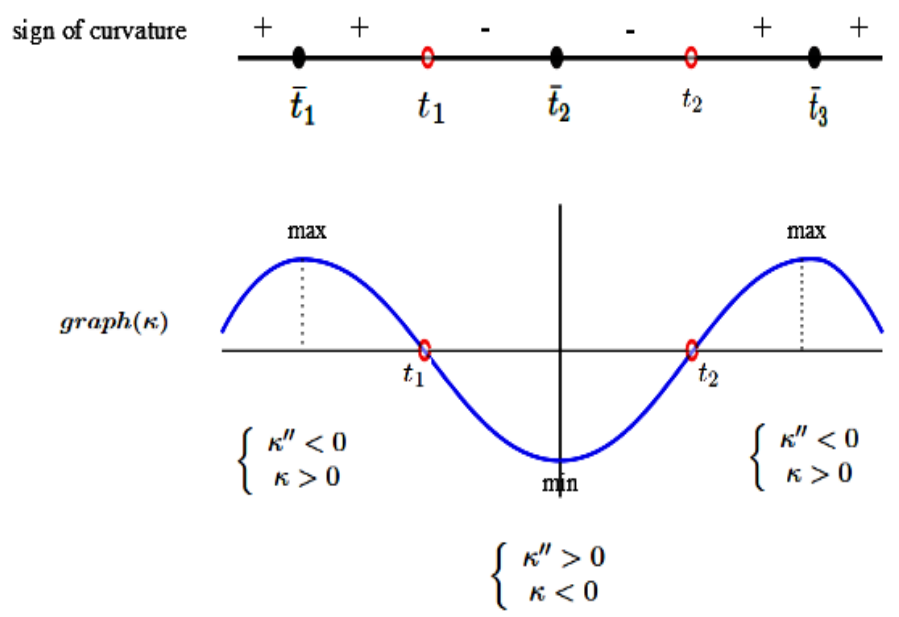

Figure 3.8 - Outward vertices in $F R S$-generic family of an ordinary cusp.

(iii) The distance squared function $D_{q}$ on $\gamma$ has an $A_{3}$-singularity at $p$ if $q=p$ and an $A_{2}$-singularity if $q \neq p$ is on the limiting normal line to $\gamma$ at $p$.

(iv) The morphism between the family of distance squared functions on $\gamma$ and that of an $\mathcal{R}^{+}$-versal deformation $t^{4}+u t^{2}+$ vt of an $A_{3}$-singularity induces a map-germ $\phi: \mathbb{R}^{2}, 0 \rightarrow \mathbb{R}^{2}, 0$ with $\phi(q)=(u, v)$, which is $\mathcal{A}$-equivalent to the cusp map-germ $(x, y) \mapsto\left(x, x y+y^{3}\right)$.

(v) For an FRS-generic family of curves $\gamma_{s}$ with $\gamma_{0}=\gamma$, the big bifurcation set of the family of distance squared functions on $\gamma$ is as in Figure 3.11, left. Its sections by $s=$ constant, which model the generic bifurcations in the evolute of $\gamma$, are as in Figure 3.11, right and Figure 3.9. One can also draw the evolute of $\gamma_{s}$ by taking into consideration the position of the vertices and inflections (Theorem 3.3) and the behavior of the evolute at the inflections.
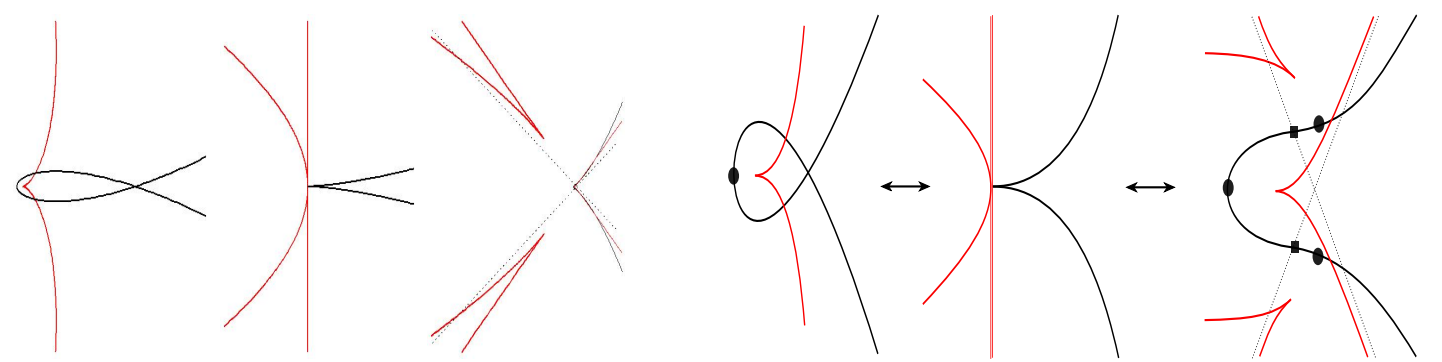

Figure 3.9 - Bifurcations of a cusp and of its full evolute, Maple figures left and and sketched figures right (normal lines at the inflections in dots).

Proof. (i) We take $\gamma$ as in (3.5) with $b_{3} \neq 0$ and $t$ varying in a small interval centred at zero. Then

$$
\kappa(t)=\frac{2\left(t \beta^{\prime \prime}(t)-\beta^{\prime}(t)\right)}{\left(4 t^{2}+\beta^{\prime}(t)^{2}\right)^{\frac{3}{2}}}=\frac{3 b_{3}+8 b_{4} t+O\left(t^{2}\right)}{4 t\left(1+\left(\frac{3}{2} b_{3} t+2 b_{4} t^{2}+O\left(t^{3}\right)\right)^{2}\right)^{\frac{3}{2}}},
$$


so that $1 / \kappa(t)$ is locally a smooth function vanishing at $t=0$. Then the proper evolute is a smooth curve parametrised by

$$
e(t)=\left(t^{2}, \beta(t)\right)+\frac{4 t^{2}+\beta^{\prime}(t)^{2}}{2\left(t \beta^{\prime \prime}(t)-\beta^{\prime}(t)\right)}\left(-\beta^{\prime}(t), 2 t\right)=\left(-t^{2}+O\left(t^{3}\right), \frac{4}{3 b_{3}} t+O\left(t^{2}\right)\right)
$$

and has ordinary tangency with the limiting normal line $x=0$.

(ii) The full evolute is the bifurcation set of the family of distance squared functions

$$
D(t, a, b)=\left(t^{2}-a\right)^{2}+(\beta(t)-b)^{2}=\beta(t)^{2}+t^{4}-2 \beta(t) b-2 a t^{2}+a^{2}+b^{2} .
$$

We have

$$
\begin{aligned}
& \frac{1}{2} D_{(a, b)}^{\prime}(t)=2 t^{3}-2 a t+\beta^{\prime}(t)(\beta(t)-b), \\
& \frac{1}{2} D_{(a, b)}^{\prime \prime}(t)=6 t^{2}-2 a+\beta^{\prime \prime}(t)(\beta(t)-b)+\beta^{\prime}(t)^{2} .
\end{aligned}
$$

From $D_{(a, b)}^{\prime \prime}(t)$ we get $a=3 t^{2}+\frac{1}{2} \beta^{\prime \prime}(t)(\beta(t)-b)+\frac{1}{2} \beta^{\prime}(t)^{2}$ and substituiting this in $D_{(a, b)}^{\prime}(t)=0$ gives

$$
t^{2}\left(4 t-\frac{\left(t \beta^{\prime \prime}(t)-\beta^{\prime}(t)\right)}{t^{2}} b+\frac{\left(t \beta^{\prime \prime}(t)-\beta^{\prime}(t)\right) \beta(t)-t \beta^{\prime}(t)^{2}}{t^{2}}\right)=0 .
$$

We get $t=0$ as a double root of (3.8) giving $a=0$ as a double curve in the bifurcation set of the family $D$. For $t \neq 0$, we can solve (3.8) in $b$ to get

$$
b=\frac{4 t^{3}+\left(t \beta^{\prime \prime}(t)-\beta^{\prime}(t)\right) \beta(t)-t \beta^{\prime}(t)^{2}}{t \beta^{\prime \prime}(t)-\beta^{\prime}(t)}
$$

This gives $(a, b)=\left(-t^{2}+O\left(t^{2}\right), \frac{4}{3 b_{3}} t+O\left(t^{2}\right)\right)$ as a parametrisation of the proper evolute. Thus, the full evolute is the union of the proper evolute together with the limiting normal line at the cusp counted twice.

(iii) From (3.7) if $a \neq 0$ then $D_{(a, b)}$ has an $A_{1}$-singularity at $t=0$. It has an $A_{2}$-singularity if $a=0$ and $b \neq 0$ and an $A_{3}$-singularity if $a=b=0$.

(iv) As $F(t, u, v)=t^{4}+u t^{2}+v t$ is an $\mathcal{R}^{+}$-versal deformation of the $A_{3}$-singularity, we have $D(t, a, b)=F(h(t, a, b), \psi(a, b))+c(a, b)$ for some germs of smooth maps $h, \psi, c$. The map-germ $\psi$ takes the bifurcation set of $D$ (i.e. the evolute) to that of $F$ (i.e., the cusp $(u, v)=$ $\left.\left(-6 t^{2}, 8 t^{3}\right)\right)$. We can calculate the 3 -jet of $\psi$ and get

$$
j^{3} \psi(a, b)=\left(-2 a-\frac{3}{2} b_{3}^{2} b^{2}-3 b_{3}^{2} b_{4} b^{3},-2 b_{3} a b-4 b_{3} b_{4} a b^{2}-b_{3}^{3} b^{3}\right) .
$$

As $b_{3} \neq 0, j^{3} \psi \sim_{\mathcal{A}^{(3)}}\left(a, a b+b^{3}\right)$; where $\mathcal{A}^{(k)}=\mathcal{A} / \mathcal{A}_{k}$, with $\mathcal{A}_{k}$ the subgroup of $\mathcal{A}$ whose elements have $k$-jets the germ of the identity. The cusp map $(a, b) \mapsto\left(a, a b+b^{3}\right)$ is 3 - $\mathcal{A}$ determined, so $\psi \sim_{\mathcal{A}}\left(a, a b+b^{3}\right)$. One can visualise the cusp map as the orthogonal projection of the surface $(b, \psi(a, b))$ in 3-space along $(1,0,0)$; see Figure 3.10. The cusp of Bif $(F)$ is the discriminant of the projection (i.e., the image of its critical set), and $B i f(D)$ is its pre-image by the cusp map $\psi$. One can see from Figure 3.10 why Bif $(D)$ (i.e., the full evolute) is as stated in (ii). 


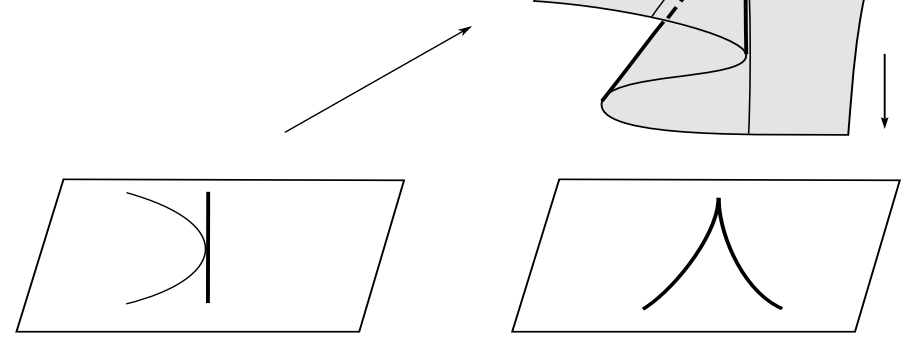

Figure 3.10 - Pre-image of the discriminant of a cusp map.

(v) Consider an FRS-generic family as in (3.6) with $\bar{b}_{1}(s)=s$. Then the big family of distance squared function is $\tilde{D}(t, a, b, s)=\left(t^{2}-a\right)^{2}+\left(\beta_{s}(t)-b\right)^{2}$. We have

$$
\begin{aligned}
\frac{1}{2} \tilde{D}_{t} & =2 t^{3}-2 a t+\beta_{s}^{\prime}(t)\left(\beta_{s}(t)-b\right), \\
\frac{1}{2} \tilde{D}_{t t} & =6 t^{2}-2 a+\beta_{s}^{\prime \prime}(t)\left(\beta_{s}(t)-b\right)+\beta_{s}^{\prime}(t)^{2}
\end{aligned}
$$

and consider the projection $\pi: \mathbb{R} \times \mathbb{R}^{2} \times \mathbb{R} \rightarrow \mathbb{R}^{2} \times \mathbb{R}$ given by $\pi(t, a, b, s)=(a, b, s)$. Then the big bifurcation set $\operatorname{Bif}(\tilde{D})$ is the image by $\pi$ of the surface $S=\left\{(t, a, b, s) \in \mathbb{R} \times \mathbb{R}^{2} \times \mathbb{R}\right.$ : $\left.\tilde{D}_{t}=\tilde{D}_{t t}=0\right\}$. The surface $\operatorname{Bif}(\tilde{D})$ is singular if and only if the kernel direction of $\pi$ (i.e. $(1,0,0,0))$ is tangent to $S$, and this occurs when $\tilde{D}_{t t t}=0$. As $\beta_{0}^{\prime \prime \prime}(0) \neq 0, \tilde{D}_{t t t}=0$ gives $b=b(s, t)$. Now $\tilde{D}_{t t}=0$ gives $a=a(s, t)$ and substituting in $\tilde{D}_{t}$ we get an equation $f(s, t)=0$ where $j^{2} f=-\frac{4}{b_{3}} t s$. Therefore, the singular set of $B i f(\tilde{D})$ consists of two curves. These curves are smooth with tangent directions along $(0,1,0)$ and $(0,0,1)$. In fact they can be parametrised by map-germs with 2-jets in the form $\left(-3 t^{2},-\frac{2}{b_{3}} t-\frac{8 b_{4}}{b_{3}^{2}} t^{2}, b_{3} t^{2}\right)$ and $\left(\frac{1}{2} s^{2}, 0, s\right)$. In particular, one branch is tangent to the plane $s=0$ and the other is transverse to it. As the tangency of the branch with the plane $s=0$ is an ordinary one $\left(b_{3} \neq 0\right)$, it intersects the planes parallels to $s=0$ at two points if $s>0$ and none if $s<0$ or vice versa (depending on the sign of $b_{3}$ ). The intersection of the other branch with the planes parallel to $s=0$ is a single point. We also observe that, away from the origin, the surface $\operatorname{Bif}(\tilde{D})$ is a cuspidal-edge along the singular curves, and is tangent to the plane $s=0$ along a smooth curve. The surface $B i f(\tilde{D})$ and its sections by the planes $s=$ constant are depicted in Figure 3.11.

\subsection{FRS-deformations of the ramphoid cusp}

As in the cusp case, we consider a stratification of the $k$-jet space $J^{k}(1,2)$. Here we also need to consider multi-local strata, so the stratification is in the multi-jet space ${ }_{2} J^{k}(1,2) \subset$ $J^{k}(1,2) \times J^{k}(1,2)$ (see for example [4] for details). The strata of interest are the ramphoid cusp stratum $(R C)$ and the following strata (occurning in 1-parameter families of curves): 


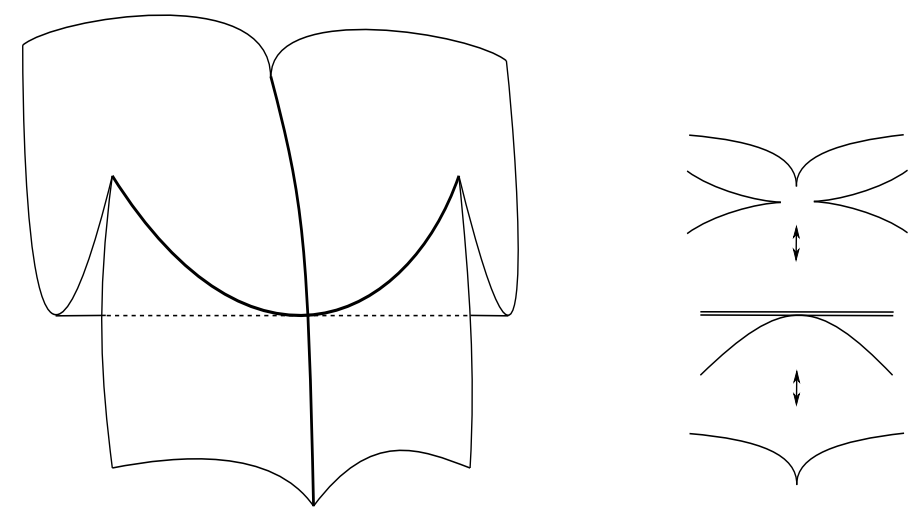

Figure 3.11 - Big bifurcation set of the family of distance squared functions on a cusp curve (left) and its sections by the planes $s=$ constant (right).

$C: \quad$ cusp singularities

$S I: \quad$ second order inflections

$I T:$ an inflection intersecting transversally another branch

Tc: two tangential branches (tacnode)

$S V:$ second order vertices

$V T:$ a vertex intersecting transversally another branch

The local strata in $J^{k}(1,2)$ give product strata in ${ }_{2} J^{k}(1,2)$, so it is enough to compute them in $J^{k}(1,2)$. Using the notation in $\S 3.2$, the $R C$ stratum is given by $a_{1}=b_{1}=a_{2} b_{3}-a_{3} b_{2}=0$ and the other strata are given by the following equations:

$$
\begin{aligned}
& C:\left\{\begin{array}{l}
a_{1}=0 \\
b_{1}=0
\end{array}\right. \\
& S I:\left\{\begin{array}{l}
a_{1} b_{2}-a_{2} b_{1}=0 \\
a_{1} b_{3}-a_{3} b_{1}=0
\end{array}\right. \\
& S V:\left\{\begin{array}{l}
-2\left(a_{1} b_{2}-a_{2} b_{1}\right)\left(a_{1} a_{2}+b_{1} b_{2}\right)+\left(a_{1}^{2}+b_{1}^{2}\right)\left(a_{1} b_{3}-a_{3} b_{1}\right)=0 \\
4\left(a_{1} b_{2}-b_{1} a_{2}\right)\left[\left(2 a_{2}+b_{2}\right) a_{1}-\left(a_{2}-2 b_{2}\right) b_{1}\right]\left[\left(2 a_{2}-b_{2}\right) a_{1}+\left(a_{2}+2 b_{2}\right) b_{1}\right] \\
-2\left(a_{1}^{2}+b_{1}^{2}\right)\left[\left(5 a_{2} b_{3}+4 b_{2} a_{3}\right) a_{1}^{2}-9\left(a_{3} a_{2}-b_{3} b_{2}\right) a_{1} b_{1}-\left(4 a_{2} b_{3}+5 b_{2} a_{3}\right) b_{1}^{2}\right] \\
+4\left(a_{1}^{2}+b_{1}^{2}\right)^{2}\left(b_{4} a_{1}-b_{1} a_{4}\right)=0
\end{array}\right.
\end{aligned}
$$

For multi-local singularities we also need to take into consideration the value of $\gamma(t)$, so for $\gamma(t)=(\alpha(t), \beta(t))$, we have the bi-jet Taylor expansion $\operatorname{map}_{2} j^{k} \phi_{\gamma}:(\mathbb{R} \times \mathbb{R},(0,0)) \rightarrow{ }_{2} J^{k}(1,2)$, with

$$
\begin{aligned}
{ }_{2} j^{k} \phi_{\gamma}\left(t_{1}, t_{2}\right)= & \left(\left(\alpha\left(t_{1}\right), \alpha^{\prime}\left(t_{1}\right), \frac{1}{2 !} \alpha^{\prime \prime}\left(t_{1}\right), \ldots ; \beta\left(t_{1}\right), \beta^{\prime}\left(t_{1}\right), \frac{1}{2 !} \beta^{\prime \prime}\left(t_{1}\right), \frac{1}{3 !} \beta^{\prime \prime \prime}\left(t_{1}\right), \ldots\right),\right. \\
& \left.\left(\alpha\left(t_{2}\right), \alpha^{\prime}\left(t_{2}\right), \frac{1}{2 !} \alpha^{\prime \prime}\left(t_{2}\right), \ldots ; \beta\left(t_{2}\right), \beta^{\prime}\left(t_{2}\right), \frac{1}{2 !} \beta^{\prime \prime}\left(t_{2}\right), \frac{1}{3 !} \beta^{\prime \prime \prime}\left(t_{2}\right), \ldots\right)\right) .
\end{aligned}
$$

Denote by $\left(\left(a_{0}, a_{1}, \ldots a_{k} ; b_{0}, b_{1}, \ldots, b_{k}\right),\left(a_{0}^{\prime}, a_{1}^{\prime}, \ldots a_{k}^{\prime} ; b_{0}^{\prime}, b_{1}^{\prime}, \ldots, b_{k}^{\prime}\right)\right)$ the coordinates in $J^{k}(1,2) \times$ $J^{k}(1,2)$. Then the multi-local strata of interest are given by the following equations: 
$I T: \quad\left\{\begin{array}{l}a_{0}-a_{0}^{\prime}=0 \\ b_{0}-b_{0}^{\prime}=0 \\ a_{1} b_{2}-a_{2} b_{1}=0\end{array}\right.$

$V T:\left\{\begin{array}{l}a_{0}-a_{0}^{\prime}=0 \\ b_{0}-b_{0}^{\prime}=0 \\ -2\left(a_{1} b_{2}-a_{2} b_{1}\right)\left(a_{1} a_{2}+b_{1} b_{2}\right)+\left(a_{1}^{2}+b_{1}^{2}\right)\left(a_{1} b_{3}-a_{3} b_{1}\right)=0\end{array}\right.$

Tc: $\left\{\begin{array}{l}a_{0}-a_{0}^{\prime}=0 \\ b_{0}-b_{0}^{\prime}=0 \\ a_{1} b_{1}^{\prime}-a_{1}^{\prime} b_{1}=0 .\end{array}\right.$

As observed in $\S 1$, we consider codimension 2 phenomena, so we take a ramphoid cusp $\mathcal{A}_{h^{-}}$ equivalent to $\left(t^{2}, t^{4}+t^{5}\right)$. For a 2-parameter family of such a ramphoid cusp to be $F R S$-generic (in any sense), it has to be an $\mathcal{A}_{e}$-versal deformation of the ramphoid cusp singularity, so we can take a local parametrisation of the family in the form

$$
\gamma_{s}(t)=\left(t^{2}, \beta(t, s)\right)=\left(t^{2}, \bar{b}_{1}(s) t+\bar{b}_{2}(s) t^{2}+\bar{b}_{3}(s) t^{3}+\bar{b}_{4}(s) t^{4}+\bar{b}_{5}(s) t^{5}+O_{s}\left(t^{6}\right)\right)
$$

with

$$
\bar{b}_{1}(s)=s_{2}, \bar{b}_{3}(s)=s_{1}, \bar{b}_{2}(0)=0, \bar{b}_{4}(0)=b_{4} \neq 0, \bar{b}_{5}(0)=b_{5} \neq 0
$$

We have now a family of bi-jet Taylor expansion map ${ }_{2} j^{k} \Phi: \mathbb{R} \times \mathbb{R} \times \mathbb{R}^{2} \rightarrow{ }_{2} J^{k}(1,2)$, given by ${ }_{2} j^{k} \Phi\left(t_{1}, t_{2}, s\right)={ }_{2} j^{k} \phi_{\gamma_{s}}\left(t_{1}, t_{2}\right)$.

The bifurcation set in the family $\gamma_{s}$ consists of the projection to the $s$-parameter space $\mathbb{R}^{2}$ of the pull-back of the stratification $S$ in ${ }_{2} J^{k}(1,2)$ by ${ }_{2} j^{k} \Phi$. We can compute the initial parts of the parametrisations or equations of the curves forming the bifurcation set in the same way as in [26].

Theorem 3.5. Let $\gamma_{s}$ be a 2-parameter $\mathcal{A}_{e}$-versal deformation of the ramphoid cusp singularity of $\gamma=\gamma_{0}$ which is $\mathcal{A}_{h}$-equivalent to $\left(t^{2}, t^{4}+t^{5}\right)$. Then the map-germ ${ }_{2} j^{k} \Phi$ is transverse (in a stratified sense) to the strata $R C, C, S I, I T, T c, S V, V T$. The bifurcation set of the family $\gamma_{s}$ consists of the origin $(R C)$ and the following curves:

$C: s_{2}=0$,

$S I: \quad s_{2}=\frac{1}{16 b_{4}^{2}} s_{1}^{3}+O\left(s_{1}^{4}\right)$,

IT: $s_{2}=-\frac{1}{4 b_{4}^{2}} s_{1}^{3}+O\left(s_{1}^{4}\right)$,

Tc: $\quad\left(s_{1}, s_{2}\right)=\left(-2 b_{5} x^{2}+O\left(\left(x^{2}\right)^{2}\right), b_{5} x^{4}+O\left(\left(x^{2}\right)^{3}\right)\right)$,

$S V: \quad\left(s_{1}, s_{2}\right)=\left(10 b_{5} x^{2}+O\left(x^{4}\right), 5 b_{5} x^{4}-16 b_{6} x^{5}+O\left(x^{6}\right)\right)$,

$V T: \quad\left(s_{1}, s_{2}\right)=\left(2 b_{5} x^{2}+O\left(x^{4}\right),-3 b_{5} x^{4}+16 b_{6} x^{5}+O\left(x^{6}\right)\right)$. 
Proof. The transversality to the strata $R C, C, T c$ follows from Mather's theorem on $\mathcal{A}_{e}$-versality implying transversality to $\mathcal{A}$-orbits. The expressions for the associated curves in the parameter space follows by similar calculations to those in [26].

For the other local strata, the tangent space to the image of $j^{k} \phi_{\gamma_{s}}(t)$ is generated by the following three vectors

$$
\begin{aligned}
\mathbf{v}_{1}=\frac{\partial}{\partial t} j^{k} \phi_{\gamma_{s}} & =\left(2,0, \ldots, 0 ; 0,0,4 b_{4}, 5 b_{5}, 6 b_{6}, \ldots\right) \\
\mathbf{v}_{2}=\frac{\partial}{\partial s_{1}} j^{k} \phi_{\gamma_{s}} & =(0,0, \ldots, 0 ; 0,0,1,0, \ldots, 0) \\
\mathbf{v}_{3}=\frac{\partial}{\partial s_{2}} j^{k} \phi_{\gamma_{s}} & =(0,0, \ldots, 0 ; 1,0, \ldots, 0)
\end{aligned}
$$

Consider the $S I$ stratum given by $a_{1} b_{2}-a_{2} b_{1}=a_{1} b_{3}-a_{3} b_{1}=0$. At $j^{k} \phi_{0}(0)$ we have $a_{2} \neq 0$, so from the first equation we get $b_{1}=a_{1} b_{2} / a_{2}$ and substituting in the second equation gives $a_{1}\left(a_{2} b_{3}-a_{3} b_{3}\right)=0$. However, $a_{1}=0$ gives $b_{1}=0$ (from the first equation) which means that we are on the cusp stratum (singular curve). Therefore, the $S I$ stratum is given by $a_{1} b_{2}-a_{2} b_{1}=a_{2} b_{3}-a_{3} b_{3}=0$, which is a smooth codimension 2 variety in $J^{k}(1,2)$. Its tangent space at $j^{k} \phi_{0}(0)$ is the intersection of the kernels of the 1 -forms $\xi_{1}=d b_{1}$ and $\xi_{2}=d b_{3}$. We have $\xi_{1}\left(\mathbf{v}_{3}\right) \neq 0$ and $\xi_{2}\left(\mathbf{v}_{2}\right) \neq 0$, so $j^{k} \Phi$ is transverse to the $S I$ stratum. This means that it intersects it on a smooth curve whose pre-image is also a smooth curve in $\left(\mathbb{R} \times \mathbb{R}^{2}, 0\right)$. We can calculate the initial jet of a parametrisation of this pre-image (see [26] for similar calculations) and find that it is given by $\left(t, s_{1}, s_{2}\right)=\left(t,-4 b_{4} t^{3}+O\left(t^{4}\right),-4 b_{4} t+O\left(t^{2}\right)\right)$. It clear now why we need the condition $b_{4} \neq 0$ (i.e., to have a ramphoid cusp $A_{h}$-equivalent to $\left(t^{2}, t^{4}+t^{5}\right)$ ) as the projection of this curve to the parameter space $\mathbb{R}^{2}$ is a regular curve if and only if $b_{4} \neq 0$ (more on this below).

The stratum $S V$ is the intersection of two singular varieties (see $\S 3.2$ for the structure of the first one). We are interested in the intersection of $S V$ with the image of $j^{k} \Phi$. As the equations of $S V$ involve only $a_{i}, b_{i}$ with $1 \leq i \leq 4$, we can work in the 4 -jet space and consider the intersection of the tangent space to the image of $j^{4} \Phi$ at $j^{4} \phi_{0}(0)$ with $S V$. From the expressions of $\mathbf{v}_{1}, \mathbf{v}_{2}, \mathbf{v}_{3}$, we can set $a_{3}=a_{4}=b_{2}=0$ (in the equations of $S V$ ). Then the sought intersection is given by the equations

$$
\begin{array}{r}
a_{1}\left(2 a_{2}^{2} b_{1}+a_{1}^{2} b_{3}+b_{1}^{2} b_{3}\right)=0 \\
-a_{2}\left(a_{1}^{2}+b_{1}^{2}\right)\left(5 a_{1}^{2}-4 b_{1}^{2}\right) b_{3}+2\left(a_{1}^{4}+b_{1}^{4}+2 a_{1}^{2} b_{1}^{2}\right) a_{1} b_{4}+2\left(b_{1}^{2}-4 a_{1}^{2}\right) a_{2}^{3} b_{1}=0
\end{array}
$$

If $a_{1}=0$ in (3.10), then (3.11) becomes $b_{1}^{3} a_{2}\left(a_{2}^{2}+2 b_{3} b_{1}\right)=0$ and as $a_{2} \neq 0$ at $j^{4} \phi_{0}(0)$, we get $b_{1}=0$. However $a_{1}=b_{1}=0$ gives the cusp stratum, so (3.10) is equivalent to $2 a_{2}^{2} b_{1}+a_{1}^{2} b_{3}+b_{1}^{2} b_{3}=0$. This is a smooth hypersurface given by $b_{1}=\phi_{1}\left(a_{1}, a_{2}, b_{3}\right)$, with $\phi_{1}$ a smooth function with a vanishing 2-jet at $j^{4} \phi_{0}(0)$. Substituting in (3.11) and taking out a factor of $a_{1}$ gives $b_{3}=\phi_{2}\left(a_{1}, a_{2}, b_{4}\right)$, with $\phi_{2}$ a smooth function with a vanishing 1 -jet at $j^{4} \phi_{0}(0)$. It follows that the intersection of $S V$ with the image of $j^{k} \Phi$ at $j^{4} \phi_{0}(0)$ is a smooth curve. We calculate the initial jet of a parametrisation of the pre-image of this curve by $j^{k} \Phi$ and find that it is given by $\left(t, s_{1}, s_{2}\right)=\left(t, 10 b_{5} t^{2}+32 b_{6} t^{3}+O\left(t^{4}\right), 5 b_{5} t^{4}+16 b_{6} t^{5}+O\left(t^{6}\right)\right)$. When projecting 
to the parameter space, we get a singular curve. The singularity is a ramphoid cusp if and only if $b_{6} \neq 0$. This is a new condition that will be require for $F R S$-genericity.

We consider now the multi-local strata $I T$. It can be seen from its defining equations that it is a stratum of codimension 3 variety. The tangent space to the image of ${ }_{2} j^{k} \Phi$ is generated by $\left(\overline{\mathbf{v}}_{1}, 0\right),\left(0, \overline{\mathbf{v}}_{1}\right),\left(\overline{\mathbf{v}}_{2}, \overline{\mathbf{v}}_{2}\right),\left(\overline{\mathbf{v}}_{3}, \overline{\mathbf{v}}_{3}\right)$ with $\overline{\mathbf{v}}_{i}=\left(0, \mathbf{v}_{i}\right)$ and the $\mathbf{v}_{i}$ 's are as above. The normal space to the codimension 2 variety given by the first two equations defining $I T$, i.e $a_{0}-a_{0}^{\prime}=b_{0}-b_{0}^{\prime}=0$ is the intersection of the kernel of the two 1-forms $\xi_{1}=d a_{0}-d a_{0}^{\prime}$ and $\xi_{2}=d b_{0}-d b_{0}^{\prime}$. The projection of the image of ${ }_{2} j^{k} \Phi$ to this space is given by $\left(\left(t_{1}^{2}, \beta_{0}\left(t_{1}\right)\right),\left(t_{2}^{2}, \beta_{0}\left(t_{2}\right)\right)\right)$. The limiting tangent space of the projected image is given by $((1,0),(0,0))$ and $((0,0),(1,0))$ so is transverse to the intersection of the kernels $\xi_{1}$ and $\xi_{2}$. The tangent space to the third equation defining IT is the kernel of $\xi_{3}=d b_{1}$. Clearly $\xi_{3}\left(\overline{\mathbf{v}}_{3}, \overline{\mathbf{v}}_{3}\right) \neq 0$. Therefore, the limiting tangent space to the image of ${ }_{2} j^{k} \Phi$ at ${ }_{2} j^{k} \phi_{0}(0,0)$ is transverse to the IT stratum. The pre-image in $\left(\mathbb{R} \times \mathbb{R} \times \mathbb{R}^{2}, 0\right)$ of the $I T$ stratum by ${ }_{2} j^{k} \Phi$ is parametrised by $\left(t_{1}, t_{2}, s_{1}, s_{2}\right)=\left(t,-t, 2 b_{4} t^{3}+O\left(t^{4}\right),-2 b_{4} t+O\left(t^{2}\right)\right)$. As $b_{4} \neq 0$, its projection to the parameter space is a regular curve.

We proceed similarly for the $V T$ stratum. Here, we find that the pre-image in $(\mathbb{R} \times \mathbb{R} \times$ $\left.\mathbb{R}^{2}, 0\right)$ of the $V T$ stratum by ${ }_{2} j^{k} \Phi$ is parametrised by $\left(t_{1}, t_{2}, s_{1}, s_{2}\right)=\left(t,-t, 2 b_{5} t^{2}+8 b_{6} t^{3}+\right.$ $\left.O\left(t^{4}\right),-3 t^{4} b_{5}-8 b_{6} t^{5}+O\left(t^{6}\right)\right)$. Here also we require $b_{6} \neq 0$ for the projection of this curve to the parameter space to have a ramphoid cusp singularity.

As a consequence of Theorem 3.5 and the comments in its proof, we define as follows $F R S$-generic families of a ramphoid cusp $A_{h}$-equivalent to $\left(t^{2}, t^{4}+t^{5}\right)$.

Definition 3.4. Let $\gamma_{0}=\left(t^{2}, \beta_{0}(t)\right)$ be smooth curve with $\beta_{0}(t)=b_{4} t^{4}+b_{5} t^{5}+b_{6} t^{6}+O\left(t^{7}\right)$ and suppose that $b_{4} \neq 0, b_{5} \neq 0$ and $b_{6} \neq 0$. A 2-parameter deformation $\gamma_{s}$ of $\gamma_{0}$ is said to be $F R S$-generic if it is an $\mathcal{A}_{e}$-versal deformation of the ramphoid cusp singularity of $\gamma_{0}$ at $t=0$.

Theorem 3.6. Let $\gamma_{s}=\left(t^{2}, \beta(t, s)\right)$ be an FRS-deformation of the curve $\gamma=\gamma_{0}$ with a ramphoid cusp singularity at $t=0$ satisfying the hypothesis in Definition 3.4. Then $\gamma_{s}$ is FRSequivalent to the model family $\left(t^{2}, t^{4}+t^{5}+t^{6}+u t^{3}+v t\right)$. The FRS-bifurcations in $\gamma_{s}$ are as shown in Figure 3.12.

Proof. It follows from Theorem 3.5 that the bifurcations sets of the family $\gamma_{s}$ and that of the model are homeomorphic. The numerator of the curvature function of $\gamma_{s}$ is given by

$$
2 s_{1}-6 s_{2} t^{2}-16\left(b_{4}+O(s)\right) t^{3}+O_{s}\left(t^{4}\right)
$$

We have a maximum of 3 inflections on any curve $\gamma_{s}$. Also as we cross the $H I$-stratum, we get 3 inflections on $\gamma_{s}$ with $s$ on one side of the $S I$-stratum and 1 inflection for $s$ on the other side of the stratum. 


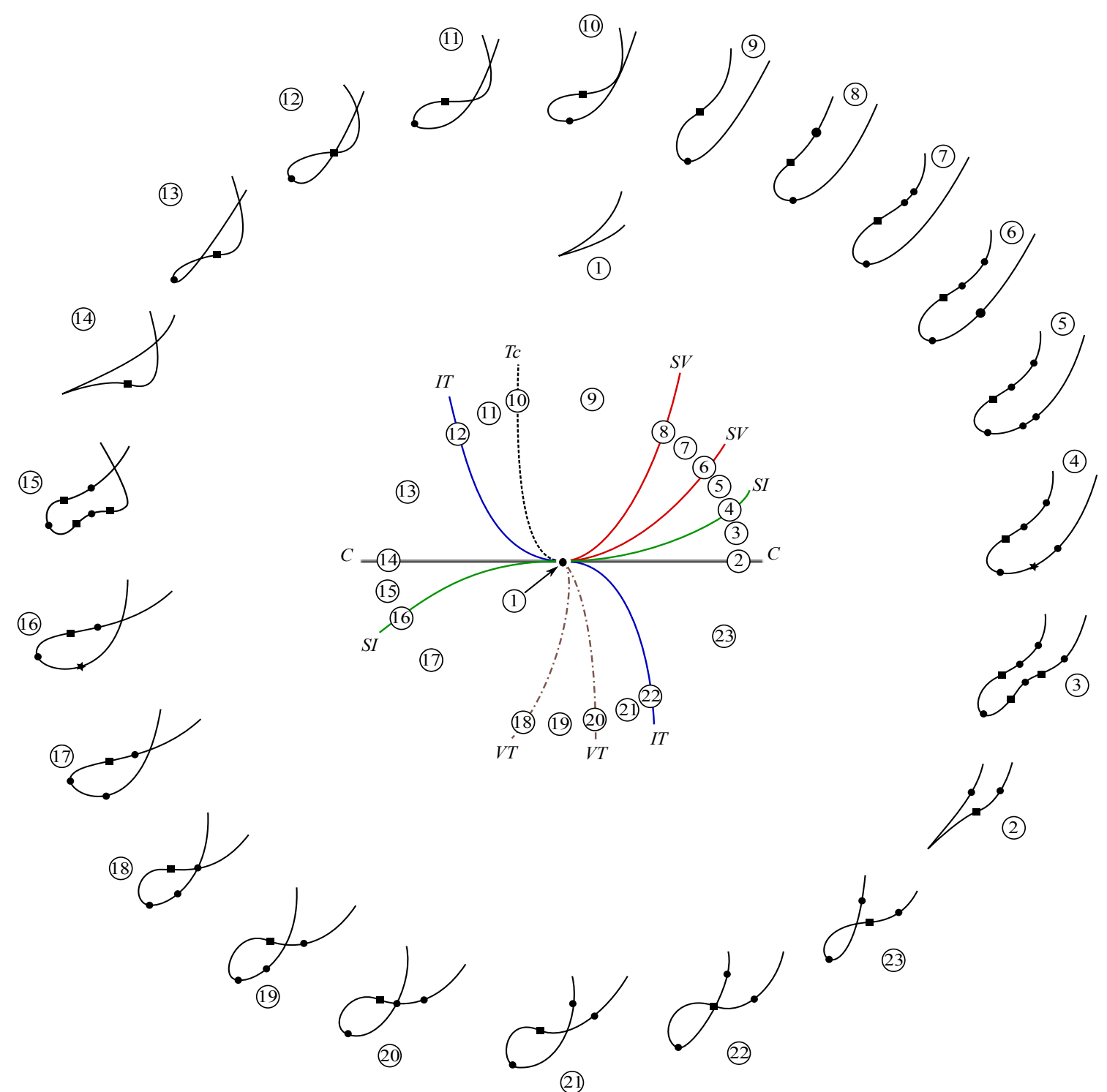

Figure 3.12 - FRS-deformations of a ramphoid cusp. First order vertices (resp. inflections) are represented by circular (resp. square) dots and second order vertices (resp. inflections) are represented by bigger circular dots (resp. stars).

Similarly, the numerator of the derivative of the curvature function of $\gamma_{s}$ is given by

$$
-24 s_{1} t+O\left(s^{2}\right) t^{2}+24 s_{2} t^{3}+O\left(s^{2}\right) t^{4}-120\left(b_{5}+O(s)\right) t^{5}+O_{s}\left(t^{6}\right) .
$$

We have a maximum of 5 vertices on any curve $\gamma_{s}$. As we cross the $S V$-stratum, we get an extra vertex on $\gamma_{s}$ with $s$ on one side of the $H V$-stratum and one vertex less for $s$ on the other side of the stratum.

To get the configuration of the inflections and vertices on $\gamma_{s}$ with $s$ in a given stratum, we use the results in $\$ 3.2$ about the $F R S$-deformations of the cusp and the fact that changes occur only as we cross a curve of the bifurcation set. Explicit calculations show that the configurations of the vertices and inflections on the cusp stratum $C$ are as in Figure 3.12 (2) and (14). It is now a matter of going around the origin in the parameter space and plotting the inflections and vertices 
on the curve as we cross the various strata of the bifurcations set.

All the above information depend only on the fact that the family is $F R S$-generic and is the same for all $F R S$-generic families. It follows from the definition of $F R S$-equivalence that any $F R S$-generic family is $F R S$-equivalent to the model family $\left(t^{2}, t^{4}+t^{5}+t^{6}+u t^{3}+v t\right)$.

Theorem 3.7. Let $\gamma_{s}=\left(t^{2}, \beta(t, s)\right)$ be an FRS-deformation of the curve $\gamma=\gamma_{0}$ with a ramphoid cusp singularity at $t=0$ satisfying the hypothesis in Definition 3.4. Then the bifurcations in its evolute are as in Figure 3.13.

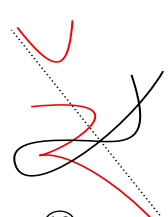

(10)
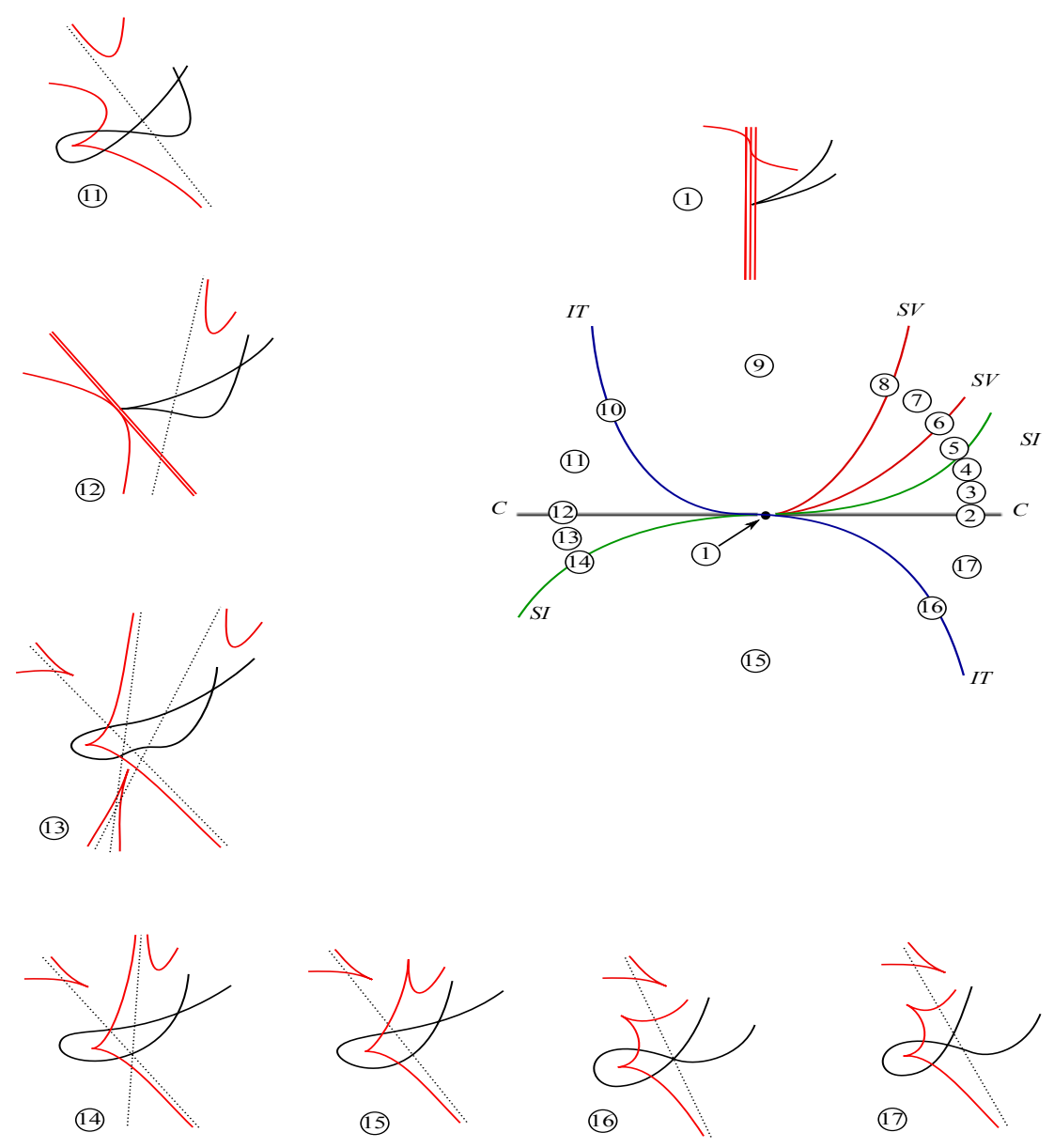

(10)
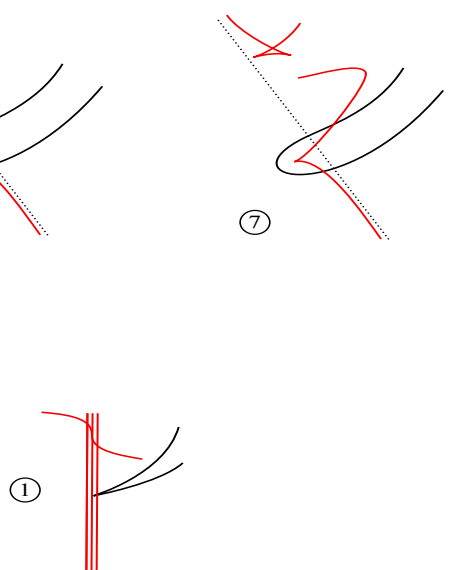

(6)
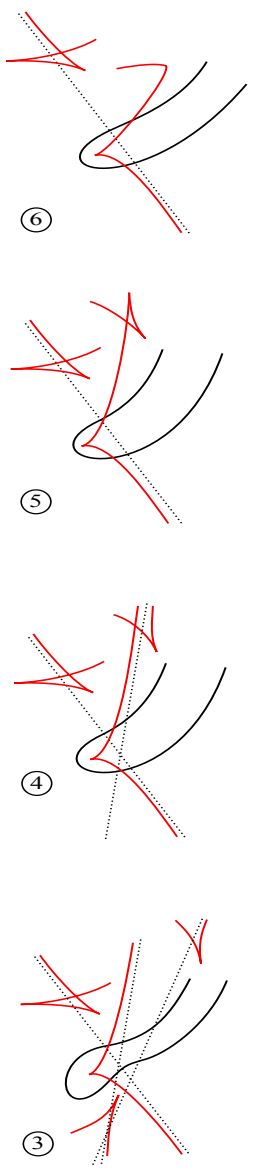

Figure 3.13 - FRS-deformations of the evolute of a ramphoid cusp (normal lines at the inflections in dots).

Proof. We start by determining the full evolute of $\gamma_{0}$. The distance squared function on $\gamma_{0}$ is given by

$$
D(t, a, b)=\left(t^{2}-a\right)^{2}+\left(\beta_{0}(t)-b\right)^{2}=\beta_{0}(t)^{2}+t^{4}-2 \beta_{0}(t) b-2 a t^{2}+a^{2}+b^{2} .
$$


Using similar calculations to those in the proof of Theorem 3.4 part (ii), we get the line $a=0$ with multiplicity 3 as part of the bifurcation set of the family $D$. The proper evolute is given by $(a, b)=\left(\frac{5 b_{5}}{8 b_{4}} t^{3}+O\left(t^{4}\right), \frac{1}{2 b_{4}}-\frac{15 b_{5}}{16 b_{4}^{2}} t+O\left(t^{2}\right)\right)$. Observe that the function $D_{(0, b)}$ has an $A_{3}$-singularity for all points $(0, b)$ along the normal line $a=0$ except at $b=\frac{1}{2 b_{4}}$ where the singularity is of type $A_{4}$.

For the rest of proof, we use the configuration of the vertices and inflections in each stratum obtained from Theorem 3.6 and Figure 3.12 and the results in $\S 3.1$ and $\S 3.2$ on $F R S$-deformations of the evolute at a cusp and at a second order inflection and vertex. For example at cusp stratum part (2) in Figure 3.12 we know from Theorem 3.6 that there is a birth of 2 vertices and one inflection. According to Theorem 3.4 the full evolute at cusp point is two lines together with a regular curve tangent to these lines. Also at inflections the evolute goes to infinity as we mentioned in \$3.1. Comparing curves (1) and (2) in Figure 3.13 we deduce that as the proper evolute of (1) has inflection at $\left(0, \frac{1}{2 b_{4}}\right)$, hence the evolute in (2) must intersect the normal line. Thereby, when (2) geting closer to (1) then two cusps in (2) joint together and construct the proper evolute in (1) as well.

Remark 3.2. In [13], F. S. Dias and F. Tari proved that for a plane curve $\gamma$ which has a singularity at point $t_{0}$, the number of vertices of $\gamma$ concentrated at the singular point satisfies

$$
V=I+\mu-2
$$

where I and $\mu$ denote the number of inflections at the singular point and the Milnor number of distance squared function $D_{c_{0}}$ of $\gamma$ respectively, where $c_{0}$ is the point in the normal direction at cusp point such that the proper evolute passes through this point. 



\section{VERTICES AND EVOLUTES OF PROJECTIONS OF SPACE CURVES}

In this chapter we apply the results in Chapter $\S 3$ to the family of orthogonal projections of space curves. Let $\gamma: I \rightarrow \mathbb{R}^{3}$ be a $C^{\infty}$ space curve and let $C^{\infty}\left(I, \mathbb{R}^{3}\right)$ denotes the set of curves. We equip this set with the Whitney $C^{\infty}$-topology.

For a given unit vector $w \in S^{2}$, the orthogonal projection of $\gamma$ along the vector $w$ is the plane curve $\alpha_{w}: I \rightarrow w^{\perp}$ given by

$$
\alpha_{w}(t)=\gamma(t)-\langle\gamma(t), w\rangle w
$$

where $w^{\perp}$ is the plane orthogonal to $w$ which we can identify with $T_{w} S^{2}$. Observe that if $w$ is tangent to $\gamma$ at $t$, then $\alpha_{w}^{\prime}(t)=0$ and if $w$ is transverse to $\gamma$ at $t$, then $\alpha_{w}(t)$ is regular at $t$. In $\S 4.2$ we consider the case when $\alpha_{w}(t)$ has vertex at $t$. The contact of the projected plane curves $\alpha_{w}(t)$ with circles gives information about the contact of $\gamma$ with circular cylinder. In $\$ 4.2 .2$ we study the contact of $\gamma$ with cylinder. In $\S 4.3$ we consider the case when $\alpha_{w}(t)$ is singular. We prove that the family of projected plane curves $\alpha_{w}(t)$ is $F R S$-generic family of an ordinary cusp and of a ramphoid cusp as well.

\subsection{Preliminaries}

Theorem 4.1 ([12]). There is a residual subset $\Omega \subset C^{\infty}\left(I, \mathbb{R}^{3}\right)$ such that if $\gamma \in \Omega$ then it is an embedding and for any $w \in S^{2}, \alpha_{w}=P_{w} \circ \gamma$ has only singularities of type $A_{0}, A_{1}, A_{2}, A_{3}, A_{4}$, $A_{5}, D_{4}, D_{5}, D_{6}$ and $\tilde{E}_{7}$.

The curves in the set $\Omega \subset C^{\infty}\left(I, \mathbb{R}^{3}\right)$ are called projection-generic by David ([12]).

For a given space curve $\gamma$ there exist Frenet frame $\{T(t), N(t), B(t)\}$ at point $t$ where $T(t)$, $N(t)$ and $B(t)$ are the tangent, normal and binormal vectors of $\gamma$ at $t$ respectively. 
We denote the curvature and torsion of $\gamma$ by $\kappa$ and $\tau$ respectively. The plane generated by $T(t)$ and $N(t)$ is called the osculating plane and denoted by $\mathcal{O}(t)$. Furthermore, the plane generated by $N(t)$ and $B(t)$ is called the normal plane. The rectifying plane is the plane generated by $T(t)$ and $B(t)$.

The following definition is given in [14].

Definition 4.1. A space curve $\gamma \in C^{\infty}\left(I, \mathbb{R}^{3}\right)$ is said to be generic if it is projection-generic and satisfies the following conditions:

(0) If $\tau(t)=0$ for some $t$ then $\tau^{\prime}(t) \neq 0$.

(1) Assume that the secant line l to $\gamma$ at two points $\gamma\left(t_{i}\right), i=1,2$, is contained in the osculating planes $\mathcal{O}\left(t_{i}\right)$ for any $i=1,2$. Then $\tau\left(t_{i}\right) \neq 0$ for any $i=1,2$.

(2) Let $l$ be a cross tangent to $\gamma$ at $\gamma\left(t_{i}\right)$ for any $i=1,2$, tangent in $\gamma\left(t_{1}\right)$. Then $\gamma^{(4)}\left(t_{1}\right) \not \subset$ $\mathcal{O}\left(t_{1}\right)$.

(3) Let $l$ be a trisecant line to $\gamma$ at three points $\gamma\left(t_{i}\right), i=1,2,3$. If $l \subset \mathcal{O}\left(t_{1}\right)$, then $\tau\left(t_{1}\right) \neq 0$ and $l \not \subset \mathcal{O}\left(t_{i}\right)$ for any $i=2,3$.

(4) Let $l$ be a trisecant line to $\gamma$ at three points $\gamma\left(t_{i}\right), i=1,2,3$. If $l$ is contained in a bitangent plane $\pi$ to $\gamma$ at two of these points, then $\pi$ does not osculate at any of the three points and $\gamma^{(3)}\left(t_{i}\right)$ is not contained in the bitangent plane.

(5) Let $l$ be a quadrisecant line to $\gamma$ at four points $\gamma\left(t_{i}\right), i=1,2,3,4$. Then, $l \not \subset \mathcal{O}\left(t_{i}\right)$ and $l \| \gamma^{(3)}\left(t_{i}\right)$ at most in two points.

In [14] it is shown that the subset of generic space curves is residual in $C^{\infty}\left(I, \mathbb{R}^{3}\right)$.

Proposition 4.1 ([14]). Let $\gamma$ be a generic space curve and let $w \in S^{2}$ be such that $w$ does not lie in any secant line of $\gamma$ passing through $\gamma\left(t_{0}\right)$. Then the germ of $P_{w} \circ \gamma$ at $t_{0}$ has a singularity of type:

$$
\begin{array}{lll}
A_{0} \quad \Leftrightarrow \quad w \notin T\left(t_{0}\right), \text { moreover, it is } A_{h} \text {-equivalent to } & A_{0_{2}} \text { if } w \notin \mathcal{O}\left(t_{0}\right), \\
& & A_{0_{3}} \text { if } w \in \mathcal{O}\left(t_{0}\right) \text { and } \tau\left(t_{0}\right) \neq 0, \\
& & A_{0_{4}} \text { if } w \in \mathcal{O}\left(t_{0}\right) \text { and } \tau\left(t_{0}\right)=0 . \\
A_{2_{3}} \Leftrightarrow w \in T\left(t_{0}\right) \text { and } \tau\left(t_{0}\right) \neq 0 . & \\
A_{4_{4}} \Leftrightarrow w \in T\left(t_{0}\right) \text { and } \tau\left(t_{0}\right)=0 . &
\end{array}
$$

The notations used in Proposition 4.1 are defined in [14]. In fact, the $A_{h}$-singularities of a generic space curve for local singularities are summarized in Table 4.1. 


\begin{tabular}{ccc}
$\mathcal{A}$-class & $A_{h}$-class & Germ \\
\hline$A_{0}$ & $A_{0_{2}}$ & $\left(t, t^{2}\right)$ \\
& $A_{0_{3}}$ & $\left(t, t^{3}\right)$ \\
& $A_{0_{4}}$ & $\left(t, t^{4}\right)$ \\
$A_{2}$ & $A_{2_{3}}$ & $\left(t^{2}, t^{3}\right)$ \\
$A_{4}$ & $A_{4_{4}}$ & $\left(t^{2}, t^{4}+t^{5}\right)$ \\
\hline
\end{tabular}

Table $4.1-A_{h}$-local singularities.

\subsection{Regular projections}

In this section we study the regular projected plane curves and their contact with circles. As we observed in Proposition 4.1, the projected plane curve $\alpha_{w}$ has an inflection at $t_{0}$ if and only if $w$ belongs to osculating plane $\mathcal{O}\left(t_{0}\right)$. Here, we consider the case when $w \notin \mathcal{O}\left(t_{0}\right)$.

If we let $w$ vary locally near the initial direction $w_{0}$, then we will get the family of projected plane curves $\alpha_{w}(t)$. Without loss of generality we suppose that the point $p$ is the origin and the initial direction is $w_{0}=(0,0,1)$. As we want to preserve the geometry of the projected curve, we are allowed only solid motions. We shall use rotations to fix the plane $z=0$ as the plane of projection. We use the spherical coordinate for the vector $w$ so we write $w=$ $(\sin (v), \cos (v) \sin (u), \cos (u) \cos (v))$ with $u, v \in \mathbb{R}$ near zero.

Let

$$
R=\left(\begin{array}{ccc}
\cos (v) & -\sin (v) \sin (u) & -\sin (v) \cos (u) \\
0 & \cos (v) \cos (u) & -\sin (u) \\
\sin (v) & \sin (u) \cos (v) & \cos (v) \cos (u)
\end{array}\right)
$$

be the matrix of rotation. Under (4.1), the projected plane curve $\alpha_{w}(t)$ is taken to

$$
\alpha_{w}(t)=\left(\begin{array}{c}
-\cos (u) \sin (v) \gamma_{3}(t)-\sin (v) \sin (u) \gamma_{2}(t)+\cos (v) \gamma_{1}(t) \\
\cos (u) \gamma_{2}(t)-\sin (u) \gamma_{3}(t) \\
0
\end{array}\right)
$$

which is a curve in the plane $z=0$.

The family of distance squared functions on the projected plane curve $\alpha_{w}(4.2)$, is $D$ : $I \times T S^{2} \rightarrow \mathbb{R}$ such that

$$
D(t, w, a, b)=\left\langle\alpha_{w}(t)-\mathbf{e}, \alpha_{w}(t)-\mathbf{e}\right\rangle, \quad \text { where } \quad \mathbf{e}=(a, b, 0) .
$$

Let $D_{(w, \mathbf{e})}(t):=D(t, w, a, b)$ be the distance squared function on $\alpha_{w}$ from the point $\mathbf{e}$ on the plane $z=0$.

\subsubsection{Evolute of projections of space curves}

The following theorem gives information about the $\mathcal{K}$-singularities of distance squared function on a generic immersed curve in $\mathbb{R}^{3}$. 
Theorem 4.2 (Theorem 4.9 of [24]). For a generic immersed curve $C$ in $\mathbb{R}^{3}$, the $\mathcal{R}$-singularities of $D_{(w, e)}$ are of type $A_{k}, k=1, \ldots, 5$.

Theorem 4.3. The family of distance squared functions $D$, is an $\mathcal{R}^{+}$-versal unfolding of $A_{1}, A_{2}, A_{3}, A_{4}, A_{5}$ local singularities of $D_{(w, \boldsymbol{e})}(t)$.

Proof. We prove here the versality of $D$ for special case when $w_{0}$ is equal to the binormal vector at $t_{0}=0$. A proof for the general case is given in Appendix A. Without loss of generality we can suppose that at $t_{0}=0, T(0)=(1,0,0), N(0)=(0,1,0)$ and $B(0)=(0,0,1)$. Applying the Serret-Frenet formulae we have

$$
\begin{aligned}
\gamma^{\prime}(0) & =(1,0,0), \\
\gamma^{\prime \prime}(0) & =(0, \kappa(0), 0), \\
\gamma^{(3)}(0) & =\left(-\kappa^{2}(0), \kappa^{\prime}(0), \kappa(0) \tau(0)\right), \\
\gamma^{(4)}(0) & =\left(-\kappa(0) \kappa^{\prime}(0), \kappa^{\prime \prime}(0)-\kappa^{3}(0)-\kappa(0) \tau^{2}(0), 2 \kappa^{\prime}(0) \tau(0)+\kappa(0) \tau^{\prime}(0)\right) .
\end{aligned}
$$

By using the Taylor expansion of $\gamma$ we get an explicit formula for $\gamma$ with respect to the frame $\{T(0), N(0), B(0)\}$;

$$
\gamma(t)=\left(\begin{array}{c}
t-\frac{1}{6} \kappa^{2} t^{3}-\frac{1}{8} \kappa \kappa^{\prime} t^{4}+\left(\frac{1}{120}\left(\tau^{2} \kappa^{2}+\kappa^{4}-4 \kappa^{\prime} \kappa-3 \kappa^{2}\right)\right) t^{5}+O\left(t^{6}\right) \\
\frac{1}{2} \kappa t^{2}+\frac{1}{6} \kappa^{\prime} t^{3}-\left(\frac{1}{24}\left(-\tau^{2} \kappa-\kappa^{3}+\kappa^{\prime \prime}\right)\right) t^{4}+\left(\frac{1}{120}\left(-3 \kappa^{\prime} \tau^{2}-6 \kappa^{\prime} \kappa^{2}-3 \tau^{\prime} \tau \kappa\right)\right) t^{5}+O\left(t^{6}\right) \\
-\frac{1}{6} \kappa \tau t^{3}+\left(\frac{1}{24}\left(2 \kappa^{\prime} \tau+\tau^{\prime} \kappa\right)\right) t^{4}+\left(\frac{1}{120}\left(-\tau^{3} \kappa-\tau \kappa^{3}+3 \kappa^{\prime} \tau^{\prime}+3 \kappa^{\prime \prime} \tau+\tau^{\prime \prime} \kappa\right)\right) t^{5}+O\left(t^{6}\right)
\end{array}\right)
$$

where $\kappa, \kappa^{\prime}, \kappa^{\prime \prime}, \tau, \tau^{\prime}$ and $\tau^{\prime \prime}$ are considered at $t_{0}=0$. Note that always we have $\kappa>0$.

One can get the conditions for $D_{(0,0, a, b)}(t)=D(t, 0,0, a, b)$ to have an $A_{k}$-singularity at $t_{0}=0$ for $k=1, \ldots, 5$ by an straightforward calculation (see Table 4.2).

To verify $\mathcal{R}^{+}$-versality of an $r$-parameter family of a singularity of type $A_{k},(k \geq 1)$, we should prove that every real polynomial $p(t)$ of degree $\leq k-1$ and without constant term can be written in the form

$$
p(t)=\sum_{i=1}^{r} c_{i} j^{k-1}\left(\frac{\partial F}{\partial x_{i}}\left(t, x_{0}\right)(0)\right)
$$

for some real constants $c_{i}$, where $x_{i}$ denote the parameters for $i=1, \ldots, r$ (see $\S 2.4$ and chapter 6 of [6] for more details).

There is an equivalent formulation of this criterion ([6]). Let $j^{k-1}\left(\frac{\partial F}{\partial x_{i}}\left(t, x_{0}\right)(0)\right)=\alpha_{1 i} t+$ $\alpha_{2 i} t^{2}+\cdots+\alpha_{k-1, i} t^{k-1}$ for $k=1, \ldots, r$. Then $F$ is $\mathcal{R}^{+}$-versal if and only if the $r \times(k-1)$ matrix of coefficients $\left[\alpha_{i j}\right]$ has rank $k-1$. 
We have

$$
\begin{aligned}
& j^{4}\left(\frac{\partial D}{\partial a}(t, 0,0, a, b)(0)\right)=-2 t+(1 / 3) \kappa^{2} t^{3}+(1 / 4) \kappa \kappa^{\prime} t^{4}, \\
& j^{4}\left(\frac{\partial D}{\partial b}(t, 0,0, a, b)(0)\right)=-\kappa t^{2}-(1 / 3) \kappa^{\prime} t^{3}+\left(-(1 / 12) \kappa \tau^{2}-(1 / 12) \kappa^{3}+(1 / 12) \kappa^{\prime \prime}\right) t^{4}, \\
& j^{4}\left(\frac{\partial D}{\partial u}(t, 0,0, a, b)(0)\right)=-(1 / 3) a \kappa \tau t^{3}+\left((1 / 12) a\left(2 \kappa^{\prime} \tau+\tau^{\prime} \kappa\right)+(1 / 3) \kappa \tau\right) t^{4}, \\
& j^{4}\left(\frac{\partial D}{\partial v}(t, 0,0, a, b)(0)\right)=-(1 / 3) b \kappa \tau t^{3}+(1 / 12) b\left(2 \kappa^{\prime} \tau+\tau^{\prime} \kappa\right) t^{4},
\end{aligned}
$$

so the coefficient matrix is

$$
\left[\alpha_{i j}\right]=\left[\begin{array}{cccc}
-2 & 0 & (1 / 3) \kappa^{2} & (1 / 4) \kappa \kappa^{\prime} \\
0 & -\kappa & (1 / 3) \kappa^{\prime} & \left.(-1 / 12) \kappa \tau^{2}-(1 / 12) \kappa^{3}+(1 / 12) \kappa^{\prime \prime}\right) \\
0 & 0 & -(1 / 3) a \kappa \tau & \left((1 / 12) a\left(2 \kappa^{\prime} \tau+\tau^{\prime} \kappa\right)+(1 / 3) \kappa \tau\right) \\
0 & 0 & -(1 / 3) b \kappa \tau & (1 / 12) b\left(2 \kappa^{\prime} \tau+\tau^{\prime} \kappa\right)
\end{array}\right] .
$$

For $k=1,2$, the family of distance squared functions is always $\mathcal{R}^{+}$-versal. For $k=3$, the coefficient matrix $\left[\alpha_{i j}\right]$ is

$$
\left[\begin{array}{cc}
-2 & 0 \\
0 & -\kappa \\
0 & 0 \\
0 & 0
\end{array}\right]
$$

which has rank 2 if and only if $\kappa \neq 0$ (this holds because we assumed that $\kappa>0$ ). Therefore $D$ is an $\mathcal{R}^{+}$-versal family of an $A_{3}$-singularity if and only if $\kappa \neq 0$. For $k=4$ the $4 \times 3$ matrix

$$
\left[\begin{array}{ccc}
-2 & 0 & \frac{1}{3} \kappa^{2} \\
0 & -\kappa & 0 \\
0 & 0 & 0 \\
0 & 0 & \frac{-1}{3} \tau
\end{array}\right]
$$

has rank 3 if and only if $\kappa \tau \neq 0$. Therefore the family of distance squared functions $D$, is an $\mathcal{R}^{+}$-versal of an $A_{4}$-singularity if and only if $\kappa \tau \neq 0$. For $k=5$ the determinant of the coefficient matrix $\left[\alpha_{i j}\right]$ is equal to $-\frac{2}{9} \kappa^{2} \tau^{2}$. Thus we conclude that $D$ is an $\mathcal{R}^{+}$-versal unfolding of an $A_{5}$-singularity if and only if $\kappa \tau \neq 0$.

\begin{tabular}{|l|l|l|}
\hline sing. & condition on $D_{(0, x)}(t)$ & versality \\
\hline$A_{1}$ & $a=0$ & always \\
\hline$A_{2}$ & $a=0, b=\frac{1}{\kappa(0)}$ & always \\
\hline$A_{3}$ & $a=0, b=\frac{1}{\kappa(0)}, \kappa^{\prime}(0)=0$ & $\kappa(0) \neq 0$ \\
\hline$A_{4}$ & $a=0, b=\frac{1}{\kappa(0)}, \kappa^{\prime}(0)=0, \kappa(0) \tau^{2}(0)=\kappa^{\prime \prime}(0)$ & $\kappa(0), \tau(0) \neq 0$ \\
\hline$A_{5}$ & $a=0, b=\frac{1}{\kappa(0)}, \kappa^{\prime}=0, \kappa(0) \tau^{2}(0)=\kappa^{\prime \prime}(0), 3 \kappa(0) \tau(0) \tau^{\prime}(0)=\kappa^{\prime \prime \prime}(0)$ & $\kappa(0), \tau(0) \neq 0$ \\
\hline
\end{tabular}

Table 4.2 - Conditions of $\mathcal{R}^{+}$-versality of the family of distance squared function on $\gamma$. 
Proposition 4.2. Let $\gamma: I \rightarrow \mathbb{R}^{3}$ be a space curve and $\alpha_{w}(t)$ be the modified orthogonal projection of $\gamma$ along the vector $w$ given by (4.2). Then,

i) The distance squared function, $D_{(w, e)}$, on $\alpha_{w}(t)$ has an $A_{1}$-singularity at $t=0$ if and only if $\alpha_{w}(0)-\boldsymbol{e}=\eta T(0) \times w$ for a non zero real number $\eta$ and a point $\boldsymbol{e}$ in the plane $z=0$.

ii) The distance squared function, $D_{(w, e)}$, on $\alpha_{w}(t)$ has an $A_{2}$-singularity at $t=0$ if and only if $\alpha_{w}(0)-\boldsymbol{e}=\eta T(0) \times w$ and $-\kappa(0) \eta\langle B(0), w\rangle=\langle T(0), w\rangle^{2}-1$.

iii) The curve $\alpha_{w}$ has an ordinary vertex at $t=0$ if and only if

$$
\left\{\begin{array}{l}
\alpha_{w}(0)-\boldsymbol{e}=\eta T(0) \times w \\
-\kappa(0) \eta\langle B(0), w\rangle=\langle T(0), w\rangle^{2}-1 \\
-\kappa^{\prime}(0) \eta\langle B(0), w\rangle+\kappa(0) \tau(0) \eta\langle N(0), w\rangle-3 \kappa(0)\langle N(0), w\rangle\langle B(0), w\rangle=0 .
\end{array}\right.
$$

Proof. We have

$$
\begin{aligned}
\frac{1}{2} D^{\prime}(t)= & \left\langle\alpha_{w}^{\prime}(t), \alpha_{w}(t)-e\right\rangle \\
\frac{1}{2} D^{\prime \prime}(t)= & \left\langle\alpha_{w}^{\prime \prime}(t), \alpha_{w}(t)-e\right\rangle+\left\langle\alpha_{w}^{\prime}(t), \alpha_{w}^{\prime}(t)\right\rangle \\
= & \left\langle\kappa(t) N(t)-\langle\kappa(t) N(t), w\rangle w, \alpha_{w}(t)-e\right\rangle+1-\langle T(t), w\rangle^{2}, \\
\frac{1}{2} D^{\prime \prime \prime}(t)= & \left\langle\kappa^{\prime}(t) N(t), \alpha_{w}(t)-e\right\rangle-\kappa^{2}(t)\left\langle T(t), \alpha_{w}(t)-e\right\rangle+\kappa(t) \tau(t)\left\langle B(t), \alpha_{w}(t)-e\right\rangle \\
& -\left\langle\kappa^{\prime}(t) N(t)-\kappa^{2}(t) T(t)+\kappa(t) \tau(t) B(t), w\right\rangle\left\langle w, \alpha_{w}(t)-e\right\rangle \\
& -3\langle\kappa(t) N(t), w\rangle\langle T(t), w\rangle .
\end{aligned}
$$

Therefore at $t=0$ we have

$$
\begin{aligned}
\frac{1}{2} D^{\prime}(0)=0 & \Leftrightarrow \alpha_{w}^{\prime}(0) \perp \alpha_{w}(0)-e \\
& \Leftrightarrow \alpha_{w}(0)-e=\eta \alpha_{w}^{\prime}{ }^{\perp}(0), \quad \text { where } \alpha_{w}^{\prime}{ }^{\perp}(0) \text { is the orthogonal vector to } \alpha_{w}^{\prime}(0) \\
& \Leftrightarrow \alpha_{w}(0)-e=\eta T(0) \times w \text { for some non zero real number } \eta
\end{aligned}
$$

Note that here $\left\langle w, \alpha_{w}^{\prime}{ }^{\perp}(0)\right\rangle=0$ and $\left\langle T(0)-\langle T(0), w\rangle w, \alpha_{w}^{\prime}{ }^{\perp}(0)\right\rangle=0$, therefore $\alpha_{w}^{\prime}{ }^{\perp}(0)=$ $T(0) \times w$. This proves (i). For (ii) we have

$$
\frac{1}{2} D^{\prime}(0)=\frac{1}{2} D^{\prime \prime}(0)=0 \Leftrightarrow\left\{\begin{array}{l}
\alpha_{w}(0)-e=\eta T(0) \times w \\
\left\langle\kappa(0) N(0)-\langle\kappa(0) N(0), w\rangle w, \alpha_{w}(0)-e\right\rangle+1-\langle T(0), w\rangle^{2}=0 .
\end{array}\right.
$$

Therefore

$$
\langle\kappa(0) N(0), \eta T(0) \times w\rangle-\langle\kappa(0) N(0), w\rangle\langle w, \eta T(0) \times w\rangle+1-\langle T(0), w\rangle^{2}=0 .
$$


Hence $\kappa(0) \eta\langle N(0), T(0) \times w\rangle=\langle T(0), w\rangle^{2}-1$. As $\langle N(0), T(0) \times w\rangle=\langle w, N(0) \times T(0)\rangle$ and $N(0) \times T(0)=-B(0)$, we have

$$
\eta=\frac{1-\langle T(0), w\rangle^{2}}{\kappa(0)\langle B(0), w\rangle}
$$

For the last part (iii) we have

$$
\frac{1}{2} D^{\prime}(0)=\frac{1}{2} D^{\prime \prime}(0)=\frac{1}{2} D^{(3)}(0)=0 \Leftrightarrow\left\{\begin{array}{l}
\alpha_{w}(0)-e=\eta T(0) \times w, \\
\eta=\frac{1-\langle T(0), w)^{2}}{\kappa(0)\langle B(0), w\rangle} \\
\left\langle\kappa^{\prime}(0) N(0), \alpha_{w}(0)-e\right\rangle-\kappa^{2}(0)\left\langle T(0), \alpha_{w}(0)-e\right\rangle \\
-\left\langle\kappa^{\prime}(0) N(0)-\kappa^{2}(0) T(0)+\kappa(0) \tau(0) B(0), w\right\rangle\left\langle w, \alpha_{w}(0)-e\right\rangle \\
-3\langle\kappa(0) N(0), w\rangle\langle T(0), w\rangle+\kappa(0) \tau(0)\left\langle B(0), \alpha_{w}(0)-e\right\rangle=0 .
\end{array}\right.
$$

By simplifying the above equalities we get

$$
\frac{1-\langle T(0), w\rangle^{2}}{\kappa\langle B(0), w\rangle}\left(-\kappa^{\prime}(0)\langle B(0), w\rangle+\kappa(0) \tau(0)\langle N(0), w\rangle\right)=3 \kappa(0)\langle N(0), w\rangle\langle T(0), w\rangle .
$$

Observation 4.1. An straightforward calculation shows that the curvature of projected plane curve $\alpha_{w}$, is given by

$$
\kappa_{\alpha_{w}}(t)=\frac{\kappa(t)\langle B(t), w\rangle}{\left(1-\langle T(t), w\rangle^{2}\right)^{\frac{3}{2}}}
$$

In $\$ 3.1$ we studied the evolute of an ordinary vertex. We observed that the deformations of a curve at a vertex $t_{0}$ of finite order can be studied using the family of distance squared function. If this family is an $\mathcal{R}^{+}$-versal deformation of an $A_{k}$-singularity, then we have a well understood model of the deformation of the vertex. As seen in Theorem 4.3 the family of distance squared function of a modified orthogonal projected plane curve $\alpha_{w}$ is $\mathbb{R}^{+}$-versal so following the discussion in $\$ 3.1$, we conclude that the family $\alpha_{w}$ of an $A_{3}$-singularity is $F R$-generic. The evolute at an ordinary vertex is as in Figure 4.1.
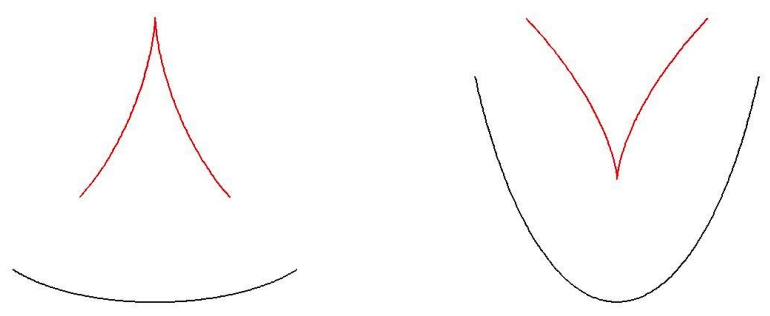

Figure 4.1 - An ordinary vertex of the projected plane curve $\alpha_{w}$.

The projected plane curve $\alpha_{w}$ has a second order vertex at $t_{0}$ if $\kappa_{\alpha_{w}}\left(t_{0}\right) \neq 0, \kappa_{\alpha_{w}}^{\prime}\left(t_{0}\right)=$ $\kappa_{\alpha_{w}}^{\prime \prime}\left(t_{0}\right)=0$ and $\kappa_{\alpha_{w}}^{\prime \prime \prime}\left(t_{0}\right) \neq 0$. The following proposition gives information about space curve $\gamma$ when its orthogonal projection has a second order vertex at $t_{0}=0$. 
Proposition 4.3. Let $\gamma: I \rightarrow \mathbb{R}^{3}$ be a space curve and $\alpha_{w}(t)$ be the modified orthogonal projection of $\gamma$ along the vector $w$ given by (4.2). Then, the plane curve $\alpha_{w}$ has a second order vertex at $t=0$ if and only if

$$
\left\{\begin{array}{l}
\alpha_{w}(0)-\boldsymbol{e}=\eta T(0) \times w \\
\eta=\frac{1-\langle T(0), w\rangle^{2}}{\kappa(0)\langle B(0), w\rangle} \\
\frac{1-T(0), w\rangle^{2}}{\kappa\langle B(0, w\rangle}\left(-\kappa^{\prime}(0)\langle B(0), w\rangle+\kappa(0) \tau(0)\langle N(0), w\rangle\right)=3 \kappa(0)\langle N(0), w\rangle\langle T(0), w\rangle \\
\left(\frac{-2 \kappa^{\prime 2}(0) \tau(0)-\kappa^{\prime}(0) \kappa(0) \tau^{\prime}(0)+\kappa(0) \kappa^{\prime \prime}(0) \tau(0)+3 \kappa^{4}(0) \tau(0)-\kappa^{2}(0) \tau^{2}(0)}{\kappa^{2}(0) \tau(0)}\right)\langle T(0), w\rangle^{2}-3 \kappa^{2}(0)\langle N(0), w\rangle^{2} \\
+\left(\frac{3 \kappa(0) \tau^{\prime}(0)+2 \kappa^{\prime}(0) \tau(0)}{\tau(0)}\right)\langle T(0), w\rangle\langle N(0), w\rangle-4 \kappa(0) \tau(0)\langle T(0), w\rangle\langle B(0), w\rangle \\
\left(\frac{2 \kappa^{\prime 2}(0) \tau(0)+\kappa(0) \kappa^{\prime}(0) \tau^{\prime}(0)+\kappa^{2}(0) \tau^{3}(0)+\kappa^{4}(0) \tau(0)-\kappa(0) \kappa^{\prime \prime}(0) \tau(0)-\kappa^{4}(0) \tau(0)}{\kappa^{2}(0) \tau(0)}\right)=0
\end{array}\right.
$$

Proof. By a direct calculation and using Proposition 4.2 one can get the proof.

Observation 4.2. Let $\gamma: I \rightarrow \mathbb{R}^{3}$ be a space curve and $\alpha_{w}(t)$ be the modified orthogonal projection of $\gamma$ along a vector $w$. If $w=B(0)$, then using Proposition 4.3 we have

$$
\alpha_{w} \text { has a second order vertex at } 0 \Leftrightarrow\left\{\begin{array}{l}
\alpha_{w}(0)-\boldsymbol{e}=-\eta N(0) \\
\eta=\frac{1}{\kappa(0)} \\
\kappa^{\prime}(0)=0 \\
\kappa^{\prime \prime}(0)=\kappa(0) \tau^{2}(0) .
\end{array}\right.
$$

According to Theorem 4.3, the family of distance squared function of an $A_{4}$-singularity is $\mathcal{R}^{+}$-versal, so using the discussion in $\S 3.1$, the projected plane curve $\alpha_{w}$ is $F R$-equivalent to the $F R$-model family. The evolute at a second order vertex is given in Figure 4.2.

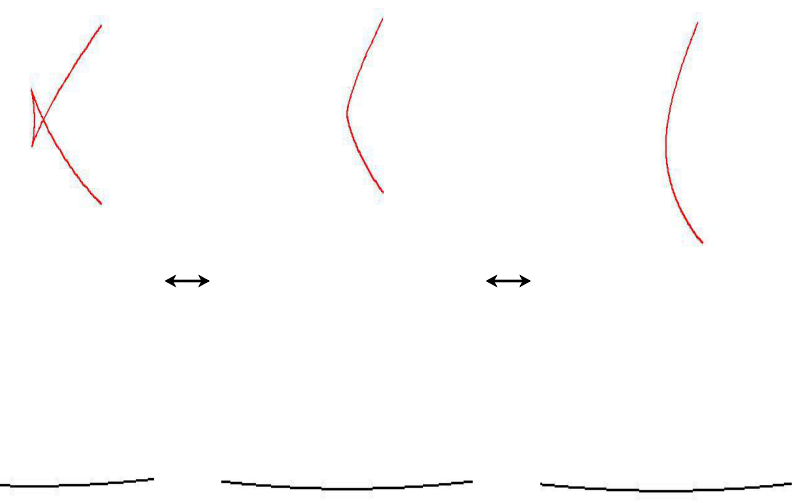

Figure 4.2 - An evolute at a second order vertex of a projected plane curve $\alpha_{w}$.

A third order vertex is a point which $\kappa_{\alpha_{w}}\left(t_{0}\right) \neq 0, \kappa_{\alpha_{w}}^{\prime}\left(t_{0}\right)=\kappa_{\alpha_{w}}^{\prime \prime}\left(t_{0}\right)=\kappa_{\alpha_{w}}^{\prime \prime \prime}\left(t_{0}\right)=0$ and $\kappa_{\alpha_{w}}^{(4)}\left(t_{0}\right) \neq 0$. A third order vertex is where the distance squared function has an $A_{5}$-singularity.

To understand the way the evolute of $\alpha_{w}$ bifurcates at an $A_{5}$-singularity of the distance squared functions as $w$ varies locally in $S^{2}$, we consider sections of the bifurcation set of the family $D$ 
by the planes $w=$ constant. The sections are in general generic (see [7] for a general study of such sections).

Theorem 4.4. Let $\gamma: I \rightarrow \mathbb{R}^{3}$ be a space curve and $\alpha_{w}$ its orthogonal projection along the unit vector $w$ as mentioned in (4.2). If $D$ denotes the family of distance squared functions on $\alpha_{w}$ with a third order vertex at $t=0$, then

i) the projection map $\pi_{1}: \operatorname{Bif}(D) \subset T S^{2} \rightarrow S^{2}$ is $\mathcal{A}$-equivalent to

$$
\begin{array}{ccc}
\pi_{2}: \operatorname{Dis}\left(A_{4}\right) \subset \mathbb{R}^{4} & \longrightarrow & \left(\mathbb{R}^{2}, 0\right) \\
\left(a_{2}, a_{3}, a_{4}, a_{5}\right) & \longmapsto & \left(a_{2}, a_{3} \pm a_{5}\right)
\end{array}
$$

where

$$
\operatorname{Dis}\left(A_{4}\right)=\left\{\left(a_{2}, a_{3}, a_{4}, a_{5}\right) \in \mathbb{R}^{4} \mid t^{5}+a_{2} t^{3}+a_{3} t^{2}+a_{4} t+a_{5}=0 \text { has a real repeated root }\right\}
$$

is the discriminant of an $\mathbb{R}$-versal unfolding of an $A_{4}$-singularity,

ii) the projection map $\pi_{1}$ is generic,

iii) the way the evolute of $\alpha_{w_{0}}$ bifurcates at a third order vertex when $w$ varies in $S^{2}$ near $w_{0}$, is shown in Figure 4.3.

Proof. Write $\gamma(t)=\left(\gamma_{1}(t), \gamma_{2}(t), \gamma_{3}(t)\right)$. We can make changes of coordinate if necessary and set $p=\gamma(0)=0$ and $w_{0}=(0,0,1) \notin T_{0} \gamma$. Thus

$$
\begin{aligned}
& \gamma_{1}(t)=x_{1} t+x_{2} t^{2}+x_{3} t^{3}+x_{4} t^{4}+x_{5} t^{5}+O\left(t^{6}\right), \\
& \gamma_{2}(t)=y_{1} t+y_{2} t^{2}+y_{3} t^{3}+y_{4} t^{4}+y_{5} t^{5}+O\left(t^{6}\right), \\
& \gamma_{3}(t)=z_{1} t+z_{2} t^{2}+z_{3} t^{3}+z_{4} t^{4}+z_{5} t^{5}+O\left(t^{6}\right),
\end{aligned}
$$

such that $\left(x_{1}, y_{1}\right) \neq(0,0)$. Now, consider the family of projected plane curves $\alpha_{w}$ as in (4.2) and let $D$ denote the family of distance squared functions on $\alpha_{w}$ at an $A_{5}$-singularity. We proved in Theorem 4.3 that the family $D$ is an $\mathcal{R}^{+}$-versal unfolding of an $A_{5}$-singularity. According to Proposition 2.1 there exists a germ of a diffeomorphism $\phi$ such that $\phi(B i f(D))=\operatorname{Bif}(G)$, where $G$ is an $\mathcal{R}^{+}$-versal model unfolding of an $A_{5}$-singularity. Moreover, we know from Remark 2.3 that there exists a germ of diffeomorphism $\psi$ such that $\psi(\operatorname{Bif}(G))=\operatorname{Dis}(F)$, where $F$ denotes the $\mathcal{R}$-versal model unfolding of an $A_{4}$-singularity. Therefore, the germ of diffeomorphism $\beta:=\psi \circ \phi$ takes $\operatorname{Bif}(D)$ to $\operatorname{Dis}(F)$. Using the diffeomorphism $\beta$ one can prove that the projection maps $\pi_{1}$ and $\pi_{2}$ are $\mathcal{A}$-equivalent (all calculation is given in Appendix B).

Bruce and Giblin listed in [7] all smooth stable map-germs $\pi:\left(\mathbb{R}^{k} \times \mathbb{R}^{n}, \operatorname{Dis}\left(A_{k}\right)\right) \rightarrow\left(\mathbb{R}^{2}, 0\right)$. For $k=4$ and $n=0$ they proved in Theorem 3.1 in [7] that $\pi_{2}$ is equivalent to the map 
$\left(a_{2}, a_{3}, a_{4}, a_{5}\right) \mapsto\left(a_{2}, a_{3} \pm a_{5}\right)$. As $\pi_{1} \sim_{\mathcal{A}} \pi_{2}$, so at an $A_{5}$-singularity of the distance squared function, the map $\pi_{1}: \operatorname{Bif}(D) \subset T S^{2} \rightarrow S^{2}$ is generic and this proves (ii).

For the rest of the proof we consider an $\mathcal{R}$-versal unfolding of an $A_{4}$-singularity and draw the bifurcation of its discriminant on a fixed plane $(u, v)=\left(a_{2}, a_{3}+a_{5}\right)$ (similar calculations hold for "-" case). To do this we shall find all strata which appear in the discriminant of $F$.

The swallowtail stratum $(S W)$ : This happens when the family of distance squared functions has an $A_{4}$-singularity. Let $F=t^{5}+a_{2} t^{3}+a_{3} t^{2}+a_{4} t+a_{5}$ be an $\mathcal{R}$-versal family of an $A_{4}$-singularity. To have a swallowtail singularity we need $\frac{\partial F}{\partial t}=\frac{\partial^{2} F}{\partial t^{2}}=\frac{\partial^{3} F}{\partial t^{3}}=0$. Therefore

$$
\begin{aligned}
\frac{\partial F}{\partial t} & =5 t^{4}+3 a_{2} t^{2}+2 a_{3} t+a_{4}=0, \\
\frac{\partial^{2} F}{\partial t^{2}} & =20 t^{3}+6 a_{2} t+2 a_{3}=0, \\
\frac{\partial^{3} F}{\partial t^{3}} & =60 t^{2}+6 a_{2}=0 .
\end{aligned}
$$

Hence $a_{2}=-10 t^{2}, a_{3}=20 t^{3}, a_{4}=-15 t^{4}$ and by substituting in $F=0$ we get $a_{5}=4 t^{5}$. Thus the swallowtail stratum is given by

$$
(u, v)=\left(a_{2}, a_{3}+a_{5}\right)=\left(-10 t^{2}, 20 t^{3}+4 t^{5}\right),
$$

which is a curve with cusp singularity at $t=0$.

The $A_{1} A_{2}$-stratum ( $A 1 A 2$ ): This occurs when there exist $t_{1} \neq t_{2}$ such that $F\left(t_{1}\right)=F\left(t_{2}\right)=0$ and $F$ has an $A_{2}$-singularity at $t_{2}$ and an $A_{1}$-singularity at $t_{1}$. Therefore,

$$
\begin{aligned}
F\left(t_{2}\right) & =t_{2}^{5}+a_{2} t_{2}^{3}+a_{3} t_{2}^{2}+a_{4} t_{2}+a_{5}=0, \\
\frac{\partial F}{\partial t}\left(t_{2}\right) & =5 t_{2}^{4}+3 a_{2} t_{2}^{2}+2 a_{3} t_{2}+a_{4}=0, \\
\frac{\partial^{2} F}{\partial t^{2}}\left(t_{2}\right) & =20 t_{2}^{3}+6 a_{2} t_{2}+2 a_{3}=0, \\
F\left(t_{1}\right) & =t_{1}^{5}+a_{2} t_{1}^{3}+a_{3} t_{1}^{2}+a_{4} t_{1}+a_{5}=0, \\
\frac{\partial F}{\partial t}\left(t_{1}\right) & =5 t_{1}^{4}+3 a_{2} t_{1}^{2}+2 a_{3} t_{1}+a_{4}=0 .
\end{aligned}
$$

We get $a_{3}=-10 t_{2}^{3}-3 a_{2} t_{2}, a_{4}=15 t_{2}^{4}+3 a_{2} t_{2}^{2}$ and $a_{5}=-6 t_{2}^{5}-a_{2} t_{2}^{3}$. Also by using Equations (4.8) and (4.5) we have

$$
5\left(t_{1}^{4}-t_{2}^{4}\right)+3 a_{2}\left(t_{1}^{2}-t_{2}^{2}\right)+2 a_{3}\left(t_{1}-t_{2}\right)=0 .
$$

This implies that $a_{2}=-(5 / 3) t_{1}^{2}-(10 / 3) t_{2} t_{1}-5 t_{2}^{2}$. In addition, Equations (4.7) and (4.4) give

$$
t_{1}^{5}-t_{2}^{5}+a_{2}\left(t_{1}^{3}-t_{2}^{3}\right)+a_{3}\left(t_{1}^{2}-t_{2}^{2}\right)+a_{4}\left(t_{1}-t_{2}\right)=0 .
$$

Thus $t_{2}=-(2 / 3) t_{1}$. This implies that $a_{2}=-(5 / 3) t_{1}^{2}, a_{3}=-(10 / 27) t_{1}^{3}, a_{4}=(20 / 27) t_{1}^{4}$ and $a_{5}=(8 / 27) t_{1}^{5}$. Therefore the trace of the $A_{1} A_{2}$-stratum on the plane $(u, v)=\left(a_{2}, a_{3}+a_{5}\right)$ is a curve given by

$$
\left(-(5 / 3) t_{1}^{2},-(10 / 27) t_{1}^{3}+(8 / 27) t_{1}^{5}\right)
$$

which is a cusp. 


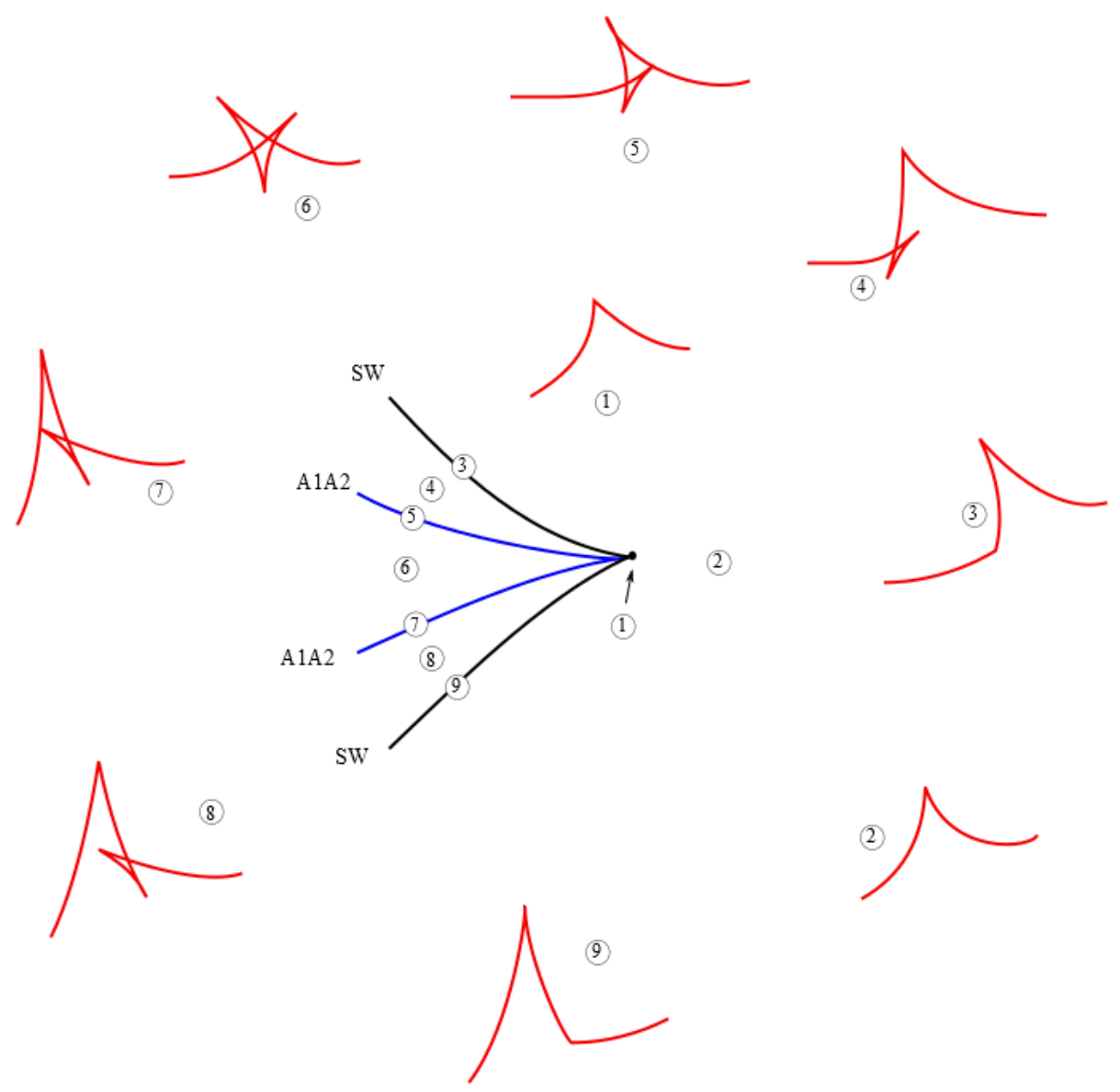

Figure 4.3 - Bifurcations in the evolute at a third order vertex.

Observation 4.3. When we consider the "-" case in Theorem 4.4 one can observe that the stratification in "-" case is homeomorph to stratification in "+" case, so they are FR-equivalent. Therefore at a third order vertex we have one FR-model family. Also note that the cusp at $u=v=0$ in Figure 4.3 is (4-5)-cusp.

Remark 4.1. In [9], Brocker and Lander drew the bifurcation diagrams for some simple singularities, including $A_{5}$, (see Figure 4.4). But according to Proposition 3.2 in [7], the authors of [9] did not consider the generic case. Also in that book they have shown that the $A_{1} A_{2}$-curve is a line, but in fact it is a curve with a cusp singularity as proved in Theorem 4.4.

\subsubsection{Projections of space curves and their contact with cylinder}

In Example 2.2 we studied the distance squared function on the projected plane curve $\alpha_{w}$. The purpose of this section is to find a relation between the contact of $\alpha_{w}$ with circles and contact of space curve $\gamma$ with circular cylinders. (see Theorem 4.5). Also some special cases have been considered in Propositions 4.5 and 4.6. 


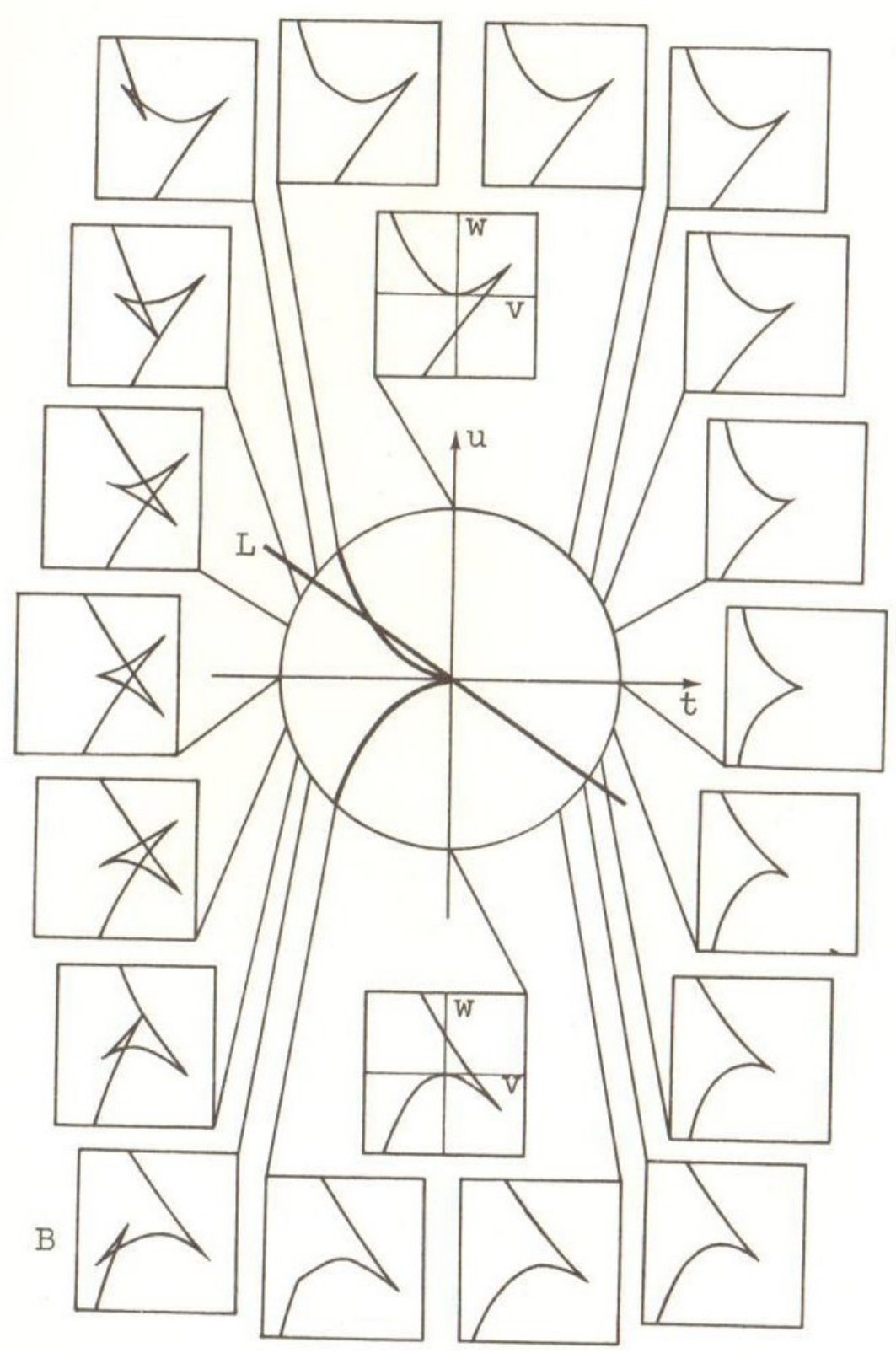

Figure 4.4 - Bifurcation diagram on the book of Brocker and Lander [9].

For a unit vector $w$, let $L$ denotes the line passing through a point $\mathbf{c} \in T_{w} S^{2}$. We set $\Delta=$ $\left\{(\mathbf{c}, w) \in \mathbb{R}^{3} \times S^{2} \mid\langle\mathbf{c}, w\rangle=0\right\}$. We define a family of functions

$$
\begin{gathered}
\mathcal{C}: \mathbb{R}^{3} \times \Delta \rightarrow \mathbb{R} \\
\mathcal{C}(p, \mathbf{c}, w)=\langle p-\langle p-\mathbf{c}, w\rangle w-\mathbf{c}, p-\langle p-\mathbf{c}, w\rangle w-\mathbf{c}\rangle .
\end{gathered}
$$

The equation $\mathcal{C}(p, \mathbf{c}, w)=r^{2} \quad(r>0)$ defines the circular cylinder formed by points $p$ at a distance $r$ from the line $L$. We denote this cylinder by $\mathcal{C}(\mathbf{c}, r)$.

Let $\gamma: I \rightarrow \mathbb{R}^{3}$ be a space curve. We define a family of functions $G: I \times \Delta \rightarrow \mathbb{R}$ by

$$
G(t, \mathbf{c}, w)=\mathcal{C}(\gamma(t), \mathbf{c}, w)
$$


The contact of the space curve $\gamma$ with cylinder is measured by singularities of germ of functions $g(t)=G(t, \mathbf{c}, w)$ for $(\mathbf{c}, w) \in \Delta$.

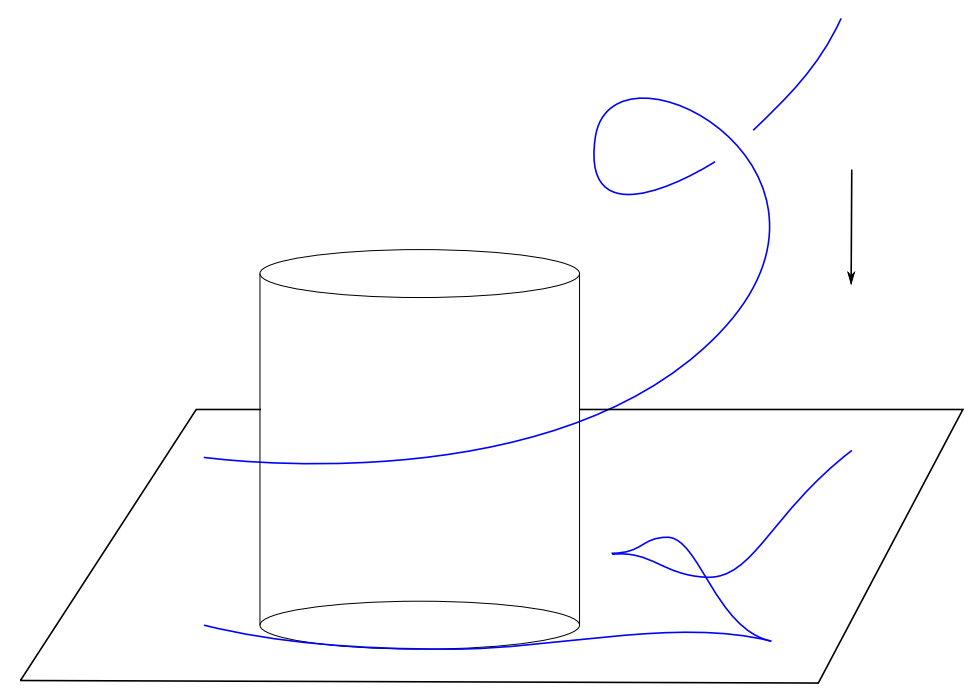

Figure 4.5 - Contact of space curves with cylinder.

Proposition 4.4. The following statements holds.

i) The cylinder $\mathcal{C}(\boldsymbol{c}, r)$ has an $A_{\geq 1}$-contact with the space curve $\gamma$ at $t$ if and only if $\alpha_{w}(t)-$ $\boldsymbol{c}=\lambda N(t)+\mu B(t)$ for some real numbers $\lambda$ and $\mu$.

ii) The cylinder $\mathcal{C}(\boldsymbol{c}, r)$ has an $A_{\geq 2}$-contact with the space curve $\gamma$ at $t$ if and only if

$$
\left\{\begin{array}{l}
\alpha_{w}(t)-\boldsymbol{c}=\lambda N(t)+\mu B(t), \\
\lambda=\frac{1}{\kappa(t)}\left(\langle T(t), w\rangle^{2}-1\right) .
\end{array}\right.
$$

iii) The cylinder $\mathcal{C}(\boldsymbol{c}, r)$ has an $A_{\geq 3}$-contact with the space curve $\gamma$ at $t$ if and only if

$$
\left\{\begin{array}{l}
\alpha_{w}(t)-\boldsymbol{c}=\lambda N(t)+\mu B(t), \\
\lambda=\frac{1}{\kappa(t)}\left(\langle T(t), w\rangle^{2}-1\right), \\
\mu=\frac{1}{\kappa(t) \tau(t)}\left(3 \kappa(t)\langle T(t), w\rangle\langle N(t), w\rangle-\frac{\kappa^{\prime}(t)}{\kappa(t)}\left(\langle T(t), w\rangle^{2}-1\right)\right) .
\end{array}\right.
$$

Proof. By a direct calculation we get $\frac{1}{2} g^{\prime}(t)=\left\langle T(t), \alpha_{w}(t)-\mathbf{c}\right\rangle$. Therefore, $\frac{1}{2} g^{\prime}(t)=0 \Leftrightarrow$ $\alpha_{w}(t)-\mathbf{c}=\lambda N(t)+\mu B(t)$. Also we have

$$
\frac{1}{2} g^{\prime \prime}(t)=\left\langle T^{\prime}(t), \alpha_{w}(t)-\mathbf{c}\right\rangle+\left\langle T(t), \alpha_{w}^{\prime}(t)\right\rangle
$$

Thus

$$
\frac{1}{2} g^{\prime}(t)=\frac{1}{2} g^{\prime \prime}(t)=0 \Leftrightarrow \alpha_{w}(t)-\mathbf{c}=\lambda N(t)+\mu B(t) \text { and } \lambda=\frac{1}{\kappa(t)}\left(\langle T(t), w\rangle^{2}-1\right) .
$$


Finally, the function $g$ has an $A_{\geq 3}$ singularity at $t$ if and only if

$$
\frac{1}{2} g^{\prime}(t)=\frac{1}{2} g^{\prime \prime}(t)=\frac{1}{2} g^{\prime \prime \prime}(t)=0 \Leftrightarrow\left\{\begin{array}{l}
\alpha-\mathbf{c}=\lambda N(t)+\mu B(t), \\
\lambda=\frac{1}{\kappa(t)}\left(\langle T(t), w\rangle^{2}-1\right), \\
\mu=\frac{1}{\kappa(t) \tau(t)}\left(3 \kappa(t)\langle T(t), w\rangle\langle N(t), w\rangle-\frac{\kappa^{\prime}(t)}{\kappa(t)}\left(\langle T(t), w\rangle^{2}-1\right)\right) .
\end{array}\right.
$$

Theorem 4.5. The space curve $\gamma$ has an $A_{k}$-contact with the cylinder $\mathcal{C}(\boldsymbol{c}, r)$ at $t$ if and only if its orthogonal projection $\alpha_{w}$ along the vector $w$ has an $A_{k}$-contact with the circle $C(\boldsymbol{e}, r)$.

Proof. One can prove this theorem by a direct calculation. Note that, the contact of space curve $\gamma$ with cylinder and the contact of plane curve $\alpha_{w}$ with circles are $\mathcal{R}$-equivalent.

In the following propositions we study some spacial cases when the vector $w$ belongs to rectifying or normal planes of $\gamma$ at $t=t_{0}$.

Proposition 4.5. If $w$ belongs to rectifying plane of $\gamma$ at $t=t_{0}$, then the following statements are equivalent;

i) $\kappa^{\prime}\left(t_{0}\right)=0$,

ii) g has an $A_{3}$-singularity,

iii) the axis of cylinder passes through the evolute of $\alpha_{w}$.

Proof. If $w$ belongs to rectifying plane of $\gamma(t)$ at $t_{0}$, then $\left\langle N\left(t_{0}\right), w\right\rangle=0$. Therefore, by Theorem 4.5 , the contact of the space curve with a cylinder has an $A_{3}$-singularity if and only if the contact of the projected plane curve $\alpha_{w}$ with a circle has an $A_{3}$-singularity. As $\left\langle B\left(t_{0}\right), w\right\rangle \neq 0$ and $\left\langle T\left(t_{0}\right), w\right\rangle^{2} \neq 1$, then using Proposition 4.2 the distance squared function on $\alpha_{w}$ has an $A_{3^{-}}$ singularity if and only if $\kappa^{\prime}\left(t_{0}\right)=0$.

Moreover, applying Proposition 4.4, we obtain that $g$ has an $A_{3}$-singularity if and only if

$$
\alpha_{w}\left(t_{0}\right)-\mathbf{c}=\frac{\left\langle T\left(t_{0}\right), w\right\rangle^{2}-1}{\kappa\left(t_{0}\right)} N\left(t_{0}\right)
$$

where $c=(a, b, t)$ is a parametrisation of the axis of the cylinder. Furthermore, the evolute of $\alpha_{w}(t)$ is given by

$$
e(t)=\alpha_{w}(t)-\eta T(t) \times(\langle T(t), w\rangle T(t)+\langle N(t), w\rangle N(t)+\langle B(t), w\rangle B(t)) .
$$

Therefore we have $e\left(t_{0}\right)=\alpha_{w}\left(t_{0}\right)+\frac{1-\left\langle T\left(t_{0}\right), w\right\rangle^{2}}{\kappa\left(t_{0}\right)} N\left(t_{0}\right)$ which means that the axis of the cylinder passes through the evolute of $\alpha_{w}$ at its vertex.

Proposition 4.6. If $w$ belongs to the normal plane of $\gamma(t)$ at $t_{0}$, then the following are equivalent: 
i) g has an $A_{3}$-singularity at $t_{0}$,

ii) $\kappa^{\prime}\left(t_{0}\right)\left\langle B\left(t_{0}\right), w\right\rangle=\kappa\left(t_{0}\right) \tau\left(t_{0}\right)\left\langle N\left(t_{0}\right), w\right\rangle$,

iii) the axis of the cylinder passes through the evolute of $\alpha_{w}$.

Proof. If $w$ belongs to the normal plane of $\gamma(t)$ at $t=t_{0}$ then $\left\langle T\left(t_{0}\right), w\right\rangle=0$. Using this assumption and according to Proposition 4.2 we observe that the distance squared function on $\alpha_{w}$ has an $A_{3}$-singularity if and only if $\kappa^{\prime}\left(t_{0}\right)\left\langle B\left(t_{0}\right), w\right\rangle=\kappa\left(t_{0}\right) \tau\left(t_{0}\right)\left\langle N\left(t_{0}\right), w\right\rangle$.

In addition, using Proposition 4.4, we conclude that $g$ has an $A_{3}$-singularity if and only if

$$
\begin{aligned}
\alpha_{w}(t)-c & =\lambda N(t)+\mu B(t), \\
\lambda & =\frac{-1}{\kappa(t)}, \\
\mu & =\frac{\kappa^{\prime}(t)}{\kappa^{2}(t) \tau(t)} .
\end{aligned}
$$

On the other hand, the part (iii) of Proposition 4.2 shows that $\alpha_{w}(t)-e=\eta T(t) \times w$ with $\eta=\frac{-1}{\kappa(t)\langle B(t), w\rangle}$. Substituting $w=\langle B(t), w\rangle B(t)+\langle N(t), w\rangle N(t)$ proves that the axis of the cylinder passes through the evolute of $\alpha_{w}$.

\subsection{Singular projections}

Following the general results in Chapter 3 our objective here is to verify whether the family of projection curves is FRS-equivalent to a given model family. We do this in Proposition 4.7 and Theorem 4.6.

According to Proposition 4.1 the projected plane curve $\alpha_{w}$ is singular if and only if $w \in T\left(t_{0}\right)$. We set without loss of generality $T\left(t_{0}\right)=(1,0,0)$. To find the family of projected plane curves $\alpha_{w}$, firstly we consider the orthogonal projection of the space curve $\gamma=\left(\gamma_{1}, \gamma_{2}, \gamma_{3}\right)$ along $w=\left(w_{1}, w_{2}, w_{3}\right) \in S^{2}$. Then, we apply the spherical change of coordinates $w_{1}=\sin (v) \cos (u)$, $w_{2}=\sin (v) \sin (u)$ and $w_{3}=\cos (v)$ and use rotations to fix the plane $x=0$ as the plane of projection.

More precisely the rotation matrix is of the form $R=R_{z}(-u) R_{y}(\pi / 2-v)$, where $R_{z}(\eta)$ (respectively $R_{y}(\xi)$ ) is the rotation matrix in $\mathbb{R}^{3}$ through an angle $\eta$ (resp. $\xi$ ) about the $O Z$-axis (Resp. $O Y$-axis). Consequently

$$
R=\left(\begin{array}{ccc}
\sin (v) \cos (u) & \sin (v) \sin (u) & \cos (v) \\
-\sin (u) & \cos (u) & 0 \\
-\cos (v) \cos (u) & -\cos (v) \sin (u) & \sin (v)
\end{array}\right)
$$

The plane curve $\alpha_{w}$ is the orthogonal projection of $\gamma$ along $w$ which is given by 


$$
\alpha=\left(\begin{array}{c}
\gamma_{1}-\left(\gamma_{1} w_{1}+\gamma_{2} w_{2}+\gamma_{3} w_{3}\right) w_{1} \\
\gamma_{2}-\left(\gamma_{1} w_{1}+\gamma_{2} w_{2}+\gamma_{3} w_{3}\right) v_{2} \\
\gamma_{3}-\left(\gamma_{1} w_{1}+\gamma_{2} w_{2}+\gamma_{3} w_{3}\right) v_{3}
\end{array}\right)=\left(\begin{array}{c}
\alpha_{1} \\
\alpha_{2} \\
\alpha_{3}
\end{array}\right) .
$$

Next, substitute the $w_{i}$ 's by the spherical coordinate and in virtue of the rotation (4.9), we have

$$
\tilde{P}:=\left(\begin{array}{c}
\tilde{P}_{1} \\
\tilde{P}_{2} \\
\tilde{P}_{3}
\end{array}\right)=\left(\begin{array}{ccc}
\sin (v) \cos (u) & \sin (v) \sin (u) & \cos (v) \\
-\sin (u) & \cos (u) & 0 \\
-\cos (v) \cos (u) & -\cos (v) \sin (u) & \sin (v)
\end{array}\right)\left(\begin{array}{c}
\alpha_{1} \\
\alpha_{2} \\
\alpha_{3}
\end{array}\right)
$$

where

$$
\begin{aligned}
\tilde{P}_{1}= & \alpha_{1} \sin (v) \cos (u)+\alpha_{2} \sin (v) \sin (u)+\alpha_{3} \cos (v) \\
= & \gamma_{1}\left(1-\sin (v)^{2} \cos (u)^{2}\right) \sin (v) \cos (u)-\gamma_{2} \sin (v)^{3} \cos (u)^{2} \sin (u) \\
& -\gamma_{3} \sin (v)^{2} \cos (u)^{2} \cos (v)+\gamma_{2}\left(1-\sin (v)^{2} \sin (u)^{2}\right) \sin (v) \sin (u) \\
& -\gamma_{1} \sin (v)^{3} \cos (u) \sin (u)^{2}-\gamma_{3} \sin (v)^{2} \sin (u)^{2} \cos (v) \\
& +\gamma_{3}\left(1-\cos (v)^{2}\right) \cos (v)-\gamma_{1} \cos (v)^{2} \sin (v) \cos (u)-\gamma_{2} \cos (v)^{2} \sin (v) \sin (u) \\
= & \gamma_{1}\left(\sin (v) \cos (u)-\sin (v)^{3} \cos (u)^{3}-\sin (v)^{3} \sin (u)^{2} \cos (u)-\cos (v)^{2} \sin (v) \cos (u)\right) \\
& +\gamma_{2}\left(-\sin (v)^{3} \cos (u)^{2} \sin (u)+\sin (v) \sin (u)-\sin (v)^{3} \sin (u)^{3}-\cos (v)^{2} \sin (v) \sin (u)\right) \\
& +\gamma_{3}\left(-\sin (v)^{2} \cos (v) \cos (u)^{2}-\sin (v)^{2} \cos (v) \sin (u)^{2}+\cos (v)-\cos (v)^{3}\right) \\
= & 0, \\
\tilde{P}_{2}= & \alpha_{2} \cos (u)-\alpha_{1} \sin (u) \\
= & \gamma_{1}\left(\sin (v)^{2} \cos (u)^{2} \sin (u)-\sin (u)-\sin (v)^{2} \cos (u)^{2} \sin (u)\right) \\
& +\gamma_{2}\left(\sin (v)^{2} \cos (u) \sin (u)^{2}+\cos (u)-\sin (v)^{2} \sin (u)^{2} \cos (u)\right) \\
& +\gamma_{3}\left(\frac{1}{4} \sin (2 v) \sin (2 u)-\frac{1}{4} \sin (2 v) \sin (2 u)\right) \\
= & \cos (u) \gamma_{2}-\sin (u) \gamma_{1}, \\
\tilde{P}_{3}= & -\alpha_{1} \cos (v) \cos (u)-\alpha_{2} \cos (v) \sin (u)+\alpha_{3} \sin (v) \\
= & \gamma_{1}\left(\sin (v)^{2} \cos (u)^{3} \cos (v)-\cos (v) \cos (u)+\sin (v)^{2} \cos (v) \sin (u)^{2} \cos (u)-\sin (v)^{2} \cos (v) \cos (u)\right) \\
& +\gamma_{2}\left(\sin (v)^{2} \cos (v) \cos (u)^{2} \sin (u)-\cos (v) \sin (u)+\sin (v)^{2} \cos (v) \sin (u)^{3}-\sin (v)^{2} \cos (v) \sin (u)\right) \\
& +\gamma_{3}\left(\sin (v) \cos (v)^{2} \cos (u)^{2}+\sin (v) \cos (v)^{2} \sin (u)^{2}+\sin (v)-\sin (v) \cos (v)^{2}\right) \\
= & \gamma_{3} \sin (v)-\gamma_{1} \cos (v) \cos (u)-\gamma_{2} \cos (v) \sin (u) .
\end{aligned}
$$

Here $u$ and $v$ are considered near zero and $\pi / 2$, respectively. Therefore by the change of variable $v \rightarrow v+\frac{\pi}{2}$ the rotated projection plane curve $\tilde{P}$ has the form

$$
\tilde{P}=\left[\begin{array}{c}
0 \\
\cos (u) \gamma_{2}(t)-\sin (u) \gamma_{1}(t) \\
\gamma_{3}(t) \cos (v)+\gamma_{1}(t) \sin (v) \cos (u)+\gamma_{2}(t) \sin (v) \sin (u)
\end{array}\right] .
$$

\subsubsection{The ordinary cusp}

Suppose that $\gamma_{1}(t)=x_{1} t+x_{2} t^{2}+O\left(t^{3}\right), \gamma_{2}(t)=y_{2} t^{2}+y_{3} t^{3}+O\left(t^{4}\right)$ and $\gamma_{3}(t)=z_{3} t^{3}+$ $z_{4} t^{4}+z_{5} t^{5}+O\left(t^{6}\right)$, where $x_{i}, y_{i}$ and $z_{i}$ are real numbers and $x_{1} \neq 0$. Therefore $\tilde{P}(t, 0,0)$ has 
an ordinary cusp singularity when $y_{2} z_{3} \neq 0$. According to Proposition 4.1, $\tilde{P}(t, 0,0)$ has an ordinary cusp at $t=0$ when $\tau(0) \neq 0$.

Due to Definition 3.3, to prove $F R S$-genericity of a family of plane curves with an ordinary cusp singularity, it is enough to show that this family is an $\mathcal{A}_{e}$-versal unfolding of the cusp singularity. Therefore, in the following proposition we check the $\mathcal{A}_{e}$-versality of the family of projected plane curves $\tilde{P}$ given in (4.10).

Proposition 4.7. For a given space curve $\gamma$, let $\tilde{P}$ as in (4.10) be its modified orthogonal projection along the unit direction $w$. If the plane curve $\tilde{P}_{(0,0)}(t)=\tilde{P}(t, 0,0)$ has an ordinary cusp singularity at $t=0$, then the family of modified projected plane curves $\tilde{P}$ is $\mathcal{A}_{e}$-versal. Therefore, this family is FRS-generic.

Proof. To verify $\mathcal{A}_{e}$-versality of the family $\tilde{P}$ we must prove the following assertion

$$
T A_{e} . f+\mathbb{R}\left\{\frac{\partial \tilde{P}}{\partial u}(t, 0,0), \frac{\partial \tilde{P}}{\partial v}(t, 0,0)\right\}+\mathbb{R}\{(1,0),(0,1)\}=\mathcal{E}(1,2),
$$

where $f=\left(f_{1}, f_{2}\right)=\tilde{P}_{(0,0)}(t)=\left(\gamma_{2}(t), \gamma_{3}(t)\right)$.

We have

$$
\begin{aligned}
& \frac{\partial \tilde{P}}{\partial u}(t, 0,0)=\left(-x_{1} t+O\left(t^{2}\right), 0\right), \\
& \frac{\partial \tilde{P}}{\partial v}(t, 0,0)=\left(0, x_{1} t+O\left(t^{2}\right)\right),
\end{aligned}
$$

where $x_{1} \neq 0$. An ordinary cusp is 3 - $\mathcal{A}$-finitely determined, so it is enough to work with the 3 -jet space. We have

$$
\begin{aligned}
& \frac{1}{2 y_{2}} j^{3} t^{2} \frac{\partial f}{\partial t}=\left(t^{3}, 0\right), \\
& \frac{1}{z_{3}}\left(0, f_{2}\right)=\left(0, t^{3}\right) .
\end{aligned}
$$

Hence $\mathcal{M}^{3} \subset T \mathcal{A}_{e} . f$. Furthermore, $\frac{1}{2 y_{2}} j^{2} t \frac{\partial f}{\partial t}=\left(t^{2}, 0\right)$ and $\frac{1}{y_{2}}\left(0, f_{1}\right)=\left(0, t^{2}\right)$. Therefore $\mathcal{M}^{2} \subset T \mathcal{A}_{e} . f$ and this proves the versality of $\tilde{P}$. We conclude by Definition 3.3 that the family of projected plane curves $\tilde{P}$ is $F R S$-generic (see Figure 4.6).

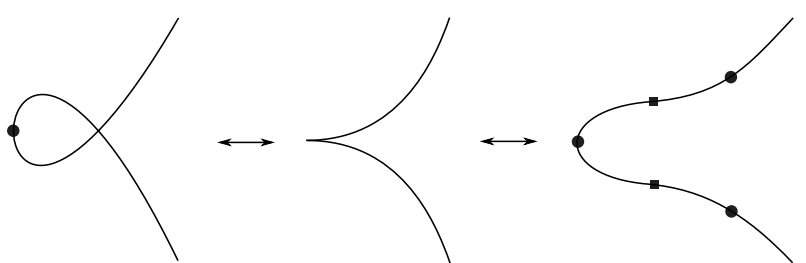

Figure 4.6-FRS-generic bifurcations of an ordinary cusp. Vertices (resp. inflections) are represented by circular (resp. square) dots. 


\subsubsection{The ramphoid cusp}

Let $\gamma_{1}(t)=x_{1} t+x_{2} t^{2}+O\left(t^{3}\right), \gamma_{2}(t)=y_{2} t^{2}+y_{3} t^{3}+O\left(t^{4}\right)$ and $\gamma_{3}(t)=z_{3} t^{3}+z_{4} t^{4}+z_{5} t^{5}+$ $O\left(t^{6}\right)$, where $x_{i}, y_{i}$ and $z_{i}$ are real numbers and $x_{1} \neq 0$.

The modified projected plane curve $\tilde{P}(t, 0,0)$ (given in (4.10)) has a ramphoid cusp singularity at $t=0$ if $y_{2} \neq 0, z_{3}=0$ and $2 y_{3} z_{4} \neq z_{5} y_{2}$. Indeed, $\tilde{P}(t, 0,0)=\left(0, \gamma_{2}(t), \gamma_{3}(t)\right)$. To avoid an ordinary cusp, the coefficient $z_{3}$ must be equal to zero. By the change of coordinate $(x, y) \mapsto\left(x, y_{2}^{2} y-z_{4} x^{2}\right)$ in the target we get that the curve $\left(\gamma_{2}(t), \gamma_{3}(t)\right)$ is $\mathcal{A}$-equivalent to $\left(y_{2} t^{2}+O\left(t^{3}\right), y_{2}\left(y_{2} z_{5}-2 y_{3} z_{4}\right) t^{5}+O\left(t^{6}\right)\right)$. This curve has a ramphoid cusp singularity if the coefficient of $t^{5}$ in the second component (i.e. $y_{2} z_{5}-2 y_{3} z_{4}$ ) is not equal to zero as well.

For a ramphoid cusp, there are three $\mathcal{A}_{h}$-classes represented by $\left(t^{2}, t^{4}+t^{5}\right),\left(t^{2}, t^{5}+t^{6}\right)$, $\left(t^{2}, t^{5}\right)$. In this thesis we study the ramphoid cusp $\mathcal{A}_{h}$-equivalent to $\left(t^{2}, t^{4}+t^{5}\right)$.

Proposition 4.8. Let $\tilde{P}_{(u, v)}(t)=\tilde{P}(t, u, v)$ be the modified orthogonal projection of the space curve $\gamma$ as in (4.10). Then $\tilde{P}$ is an $\mathcal{A}_{e}$-versal unfolding of $f(t)=\tilde{P}_{(0,0)}(t)$ if and only if $z_{4} \neq 0$.

Proof. As we mentioned in Proposition 4.7, to prove the versality of $\tilde{P}$ we need to show that

$$
T A_{e} . f+\mathbb{R}\left\{\frac{\partial \tilde{P}}{\partial u}(t, 0,0), \frac{\partial \tilde{P}}{\partial v}(t, 0,0)\right\}+\mathbb{R}\{(1,0),(0,1)\}=\mathcal{E}(1,2) .
$$

The ramphoid cusp is 5 - $\mathcal{A}$-determined we can work in 5 -jet space. We have

$$
j^{5} \frac{\partial f}{\partial t}=\left(2 y_{2} t+3 y_{3} t^{2}+4 y_{4} t^{3}+5 y_{5} t^{4}, 4 z_{4} t^{3}+5 z_{5} t^{4}+6 z_{6} t^{5}\right)
$$

which implies $j^{5} t^{4} \frac{\partial f}{\partial t}=\left(2 y_{2} t^{5}, 0\right)$ therefore $\left(t^{5}, 0\right) \in T A_{e} . f$ if and only if $y_{2} \neq 0$ (this always holds at the ramphoid cusp).

Moreover, $\left(0, f_{2}\right)=\left(0, z_{4} t^{4}+z_{5} t^{5}\right),\left(0, f_{1}^{2}\right)=\left(0, y_{2}^{2} t^{4}+2 y_{2} y_{3} t^{5}\right)$ and

$$
j^{5} t^{2} \frac{\partial f}{\partial t}=\left(2 y_{2} t^{3}+3 y_{3} t^{4}, 4 z_{4} t^{5}\right)
$$

Hence $\left(0, t^{5}\right) \in T \mathcal{A}_{e} . f$ if and only if $2 y_{3} z_{4} \neq z_{5} y_{2}$ (this also holds at the ramphoid cusp). This yields $\mathcal{M}_{5} \in T \mathcal{A}_{e} . f$.

The next step is to consider the monomials of degree 4 . Using $j^{5} t^{3} \frac{\partial f}{\partial t}=\left(2 y_{2} t^{4}, 0\right)$ and $\left(0, f_{1}^{2}\right)=\left(0, y_{2}^{2} t^{4}\right)$ one can conclude that $\mathcal{M}_{4} \in T \mathcal{A}_{e} . f$.

We have $j^{5} t^{2} \frac{\partial f}{\partial t}=\left(2 y_{2} t^{3}, 0\right)$, therefore $\left(t^{3}, 0\right) \in T \mathcal{A}_{e} . f$. The fact that $\left(f_{1}, 0\right)=\left(y_{2} t^{2}, 0\right)$ implies that $\left(t^{2}, 0\right) \in T \mathcal{A}_{e} . f$.

On the other hand, we have $\frac{\partial \tilde{P}}{\partial u}(t, 0,0)=(-t, 0), \frac{\partial \tilde{P}}{\partial v}(t, 0,0)=(0, t)$, and

$$
j^{5} \frac{\partial f}{\partial t}=\left(2 y_{2} t, 4 z_{4} t^{3}\right)=2 y_{2}(t, 0)+4 z_{4}\left(0, t^{3}\right) .
$$

Therefore one can derive that $\left(0, t^{3}\right) \in T \mathcal{A}_{e} . f+\mathbb{R}\{(t, 0),(0, t)\}$ if and only if $z_{4} \neq 0$.

Using $\left(0, f_{1}\right)=\left(0, y_{2} t^{2}+y_{3} t^{3}\right)$, we deduce that $\left(0, t^{2}\right)$ is also in $T \mathcal{A}_{e} \cdot f+\mathbb{R}\{(t, 0),(0, t)\}$ if $y_{2} \neq 0$. 
Our aim here is to prove that the family of projected plane curves $\tilde{P}(t)$, is $F R S$-equivalent to $F R S$-model family as in Theorem 3.6. To do this we need to show that the stratification of the family of orthogonal projected plane curves is homeomorph to the stratification of the FRS-model family in Theorem 3.6.

Theorem 4.6. Let $\gamma: I \rightarrow \mathbb{R}^{3}$ be a generic space curve given by $\gamma_{1}(t)=x_{1} t+x_{2} t^{2}+O\left(t^{3}\right)$, $\gamma_{2}(t)=y_{2} t^{2}+y_{3} t^{3}+O\left(t^{4}\right)$ and $\gamma_{3}(t)=z_{3} t^{3}+z_{4} t^{4}+z_{5} t^{5}+z_{6} t^{6}+O\left(t^{7}\right)$. Let $\tilde{P}$ be the modified

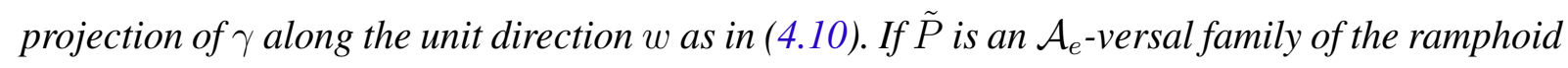
cusp $\tilde{P}(t, 0,0)$ which is $A_{h}$-equivalent to $\left(t^{2}, t^{4}+t^{5}\right)$, and $z_{6} \neq 0$ then $\tilde{P}$ is FRS-generic.

Proof. According to Proposition 4.8, the family $\tilde{P}$ is an $\mathcal{A}_{e}$-versal unfolding of the ramphoid cusp if and only if $z_{4} \neq 0$. To draw the bifurcations in the family $\tilde{P}$ we find all the strata which in the bifurcation set of the family $\tilde{P}$.

The $(R C)$-stratum: This consists of the origin, where $\tilde{P}_{(0,0)}$ has a ramphoid cusp of $A_{h}$-type equivalent to $\left(t^{2}, t^{4}+t^{5}\right)$.

The $(C)$-stratum: This is the set of $(u, v)$ where $\tilde{P}_{(u, v)}$ has a cusp singularity at some $t$.

We recall that $\tilde{P}_{(u, v)}(t)=\left(\tilde{P}_{2}(t), \tilde{P}_{3}(t)\right)$ such that $\tilde{P}_{2}(t)$ and $\tilde{P}_{2}(t)$ have the following expressions:

$$
\begin{aligned}
\tilde{P}_{2}(t) & =\cos (u) \gamma_{2}(t)-\sin (u) \gamma_{1}(t), \\
\tilde{P}_{3}(t) & =\cos (v) \gamma_{3}(t)+\sin (v) \cos (u) \gamma_{1}(t)+\sin (v) \sin (u) \gamma_{2}(t) .
\end{aligned}
$$

The curve $\tilde{P}_{(u, v)}$ is singular if and only if $\tilde{P}_{2}^{\prime}(t)=\tilde{P}_{3}^{\prime}(t)=0$, that is

$$
\left\{\begin{array}{l}
2 t-x_{1} u+O(2)=0 \\
4 t^{3}+5 t^{4}+x_{1} v+O(2)=0
\end{array}\right.
$$

The Jacobian matrix of the linear part of the system of equations $(4.12)$ at $(0,0,0)$ is given by

$$
\left[\begin{array}{cc}
-x_{1} & 0 \\
0 & x_{1}
\end{array}\right]
$$

which is invertible. Consequently, by the implicit function theorem, we can write locally $u$ and $v$ as smooth functions of $t$ with

$$
u=\frac{2}{x_{1}} t+O\left(t^{2}\right) \text { and } v=\frac{-1}{x_{1}}\left(4 t^{3}+5 t^{4}\right)+O\left(t^{5}\right) .
$$

Therefore the $(C)$-stratum is a smooth curve given by $v=\frac{-x_{1}^{2}}{2} u^{3}+O\left(u^{4}\right)$.

The $(I T)$-stratum: This happens when the height function on $\tilde{P}_{(u, v)}(t)$ has a multilocal singularity $A_{0} A_{2}$. This means that there exist $t, s \in \mathbb{R}$ with $s \neq t$, such that $\tilde{P}_{(u, v)}(t)=\tilde{P}_{(u, v)}(s)$ 
and $\kappa(s)=0$. Therefore we have

$$
\begin{aligned}
\tan (u) & =\frac{\gamma_{2}(t)-\gamma_{2}(s)}{\gamma_{1}(t)-\gamma_{1}(s)} \\
\tan (v) & =\frac{\gamma_{3}(t)-\gamma_{3}(s)}{\gamma_{1}(t)-\gamma_{1}(s)} \cdot \frac{1}{-\cos (u)-\tan (u) \sin (u)} .
\end{aligned}
$$

Replacing $u$ and $v$ obtained from (4.13) to the equation $\kappa(s)=0$, we get $f(t, s)=6 s^{3}+10 s^{2} t+$ $2 s t^{2}+2 t^{3}+g(s, t)=0$, where $g(s, t)$ has order grater than 4 . The function $f(s, t)$ is 3 - $\mathcal{R}$-finitely determined. Therefore $f \sim_{\mathcal{R}} j^{3} f$ which has singularity of type $D_{4}^{-}$at the origin. Then $f^{-1}(0)$ is diffeomorphic to $j^{3} f=2(3 s+t)(-t+s)^{2}=0$. This implies that there exists a diffeomorphism $h$ with $j^{1} h=i d$ such that $f \circ h^{-1}=j^{3} f$. Moreover $f(t, t)=0$ and the other solution is $t=-3 s$.

By using the Taylor expansion of $u$ and $v$ we get the $(I T)$-stratum given by

$$
(u, v)=\left(\frac{-2 s}{x_{1}}+O\left(s^{2}\right), \frac{20 s^{3}}{x_{1}}+O\left(s^{4}\right)\right) .
$$

The $(V T)$-stratum: This consists of $(u, v)$ where the distance squared function on $\tilde{P}_{(u, v)}$ has a multilocal singularity $A_{0} A_{3}$. Therefore there exist $t, s \in \mathbb{R}$ with $s \neq t$, such that $\tilde{P}_{(u, v)}(t)=$ $\tilde{P}_{(u, v)}(s)$ and $\kappa^{\prime}(s)=0$. By a direct calculation we get $u=\frac{\gamma_{2}(t)-\gamma_{2}(s)}{\gamma_{1}(t)-\gamma_{1}(s)}$ and $v=\frac{\gamma_{3}(t)-\gamma_{3}(s)}{\gamma_{1}(s)-\gamma_{1}(t)}$. Moreover $\kappa^{\prime}(s)=0$ if and only if

$$
\begin{aligned}
& \left(\gamma_{2}^{\prime}(s) \gamma_{3}^{\prime \prime \prime}(s)-\gamma_{2}^{\prime \prime \prime}(s) \gamma_{3}^{\prime}(s)\right)\left(\gamma_{2}^{\prime}(s)^{2}+\gamma_{3}^{\prime}(s)^{2}\right)-3\left(\gamma_{2}^{\prime}(s) \gamma_{2}^{\prime \prime}(s)+\gamma_{3}^{\prime}(s) \gamma_{3}^{\prime \prime}(s)\right)\left(\gamma_{2}^{\prime}(s) \gamma_{3}^{\prime \prime}(s)-\gamma_{2}^{\prime \prime}(s) \gamma_{3}^{\prime}(s)\right) \\
& +\left(-2\left(\gamma_{2}^{\prime}(s) \gamma_{3}^{\prime \prime \prime}(s)-\gamma_{2}^{\prime \prime \prime}(s) \gamma_{3}^{\prime}(s)\right) \gamma_{1}^{\prime}(s) \gamma_{2}^{\prime}(s)+\left(-\gamma_{1}^{\prime}(s) \gamma_{3}^{\prime \prime \prime}(s)+\gamma_{1}^{\prime \prime \prime}(s) \gamma_{3}^{\prime}(s)\right)\left(\gamma_{2}^{\prime}(s)^{2}+\gamma_{3}^{\prime}(s)^{2}\right)\right. \\
& -3\left(\gamma_{2}^{\prime}(s) \gamma_{2}^{\prime \prime}(s)+\gamma_{3}^{\prime}(s) \gamma_{3}^{\prime \prime}(s)\right)\left(-\gamma_{1}^{\prime}(s) \gamma_{3}^{\prime \prime}(s)+\gamma_{1}^{\prime \prime}(s) \gamma_{3}^{\prime}(s)\right)-3\left(-\gamma_{1}^{\prime}(s) \gamma_{2}^{\prime \prime}(s)-\gamma_{1}^{\prime \prime}(s) \gamma_{2}^{\prime}(s)\right) \\
& \left.\left(\gamma_{2}^{\prime}(s) \gamma_{3}^{\prime \prime}(s)-\gamma_{2}^{\prime \prime}(s) \gamma_{3}^{\prime}(s)\right)\right) u+\left(2\left(\gamma_{2}^{\prime}(s) \gamma_{3}^{\prime \prime \prime}(s)-\gamma_{2}^{\prime \prime \prime}(s) \gamma_{3}^{\prime}(s)\right) \gamma_{3}^{\prime}(s) \gamma_{1}^{\prime}(s)+\left(-\gamma_{1}^{\prime}(s) \gamma_{2}^{\prime \prime \prime}(s)+\gamma_{1}^{\prime \prime \prime}(s) \gamma_{2}^{\prime}(s)\right)\right. \\
& \left(\gamma_{2}^{\prime}(s)^{2}+\gamma_{3}^{\prime}(s)^{2}\right)-3\left(\gamma_{2}^{\prime}(s) \gamma_{2}^{\prime \prime}(s)+\gamma_{3}^{\prime}(s) \gamma_{3}^{\prime \prime}(s)\right)\left(-\gamma_{1}^{\prime}(s) \gamma_{2}^{\prime \prime}(s)+\gamma_{1}^{\prime \prime}(s) \gamma_{2}^{\prime}(s)\right) \\
& \left.-3\left(\gamma_{1}^{\prime}(s) \gamma_{3}^{\prime \prime}(s)+\gamma_{1}^{\prime \prime}(s) \gamma_{3}^{\prime}(s)\right)\left(\gamma_{2}^{\prime}(s) \gamma_{3}^{\prime \prime}(s)-\gamma_{2}^{\prime \prime}(s) \gamma_{3}^{\prime}(s)\right)\right) v=0 .
\end{aligned}
$$

Substituting $u$ and $v$ in to the Equation (4.14), we deduce $F(s, t)=\frac{G(s, t)}{\gamma_{1}(t)-\gamma_{1}(s)}$ such that $G(s, t)$ is divisible by $t-s$.

If we set $\hat{G}(s, t)=\frac{G(s, t)}{t-s}$, then $\hat{G}$ has a singularity of type $D_{4}^{+}$at the origin and $j^{3} \hat{G}(s, t)=$ $2(t+s)\left(3 s^{2}+t^{2}\right)$. Therefor we have $s=-t$. We apply a similar method to find higher order terms of $s$. In order to do this, we put $s=-t+\hat{t}$ and simplify the equation $\kappa^{\prime}(s)=0$. By straightforward calculations we get $\hat{t}=t^{2}+O\left(t^{3}\right)$.

Substituting $s=-t+t^{2}+O\left(t^{3}\right)$ in the expressions of $u$ and $v$ we get $u(t)=\frac{1}{x_{1}} t^{2}+O\left(t^{3}\right)$ and $v(t)=\frac{-3}{x_{1}} t^{4}+\frac{11}{2 x_{1}} t^{5}+O\left(t^{6}\right)$. Therefore the $(V T)$-stratum is a ramphoid cusp given by

$$
(u, v)=\left(\frac{1}{x_{1}} t^{2}+O\left(t^{3}\right), \frac{-3}{x_{1}} t^{4}+\frac{11}{2 x_{1}} t^{5}+O\left(t^{6}\right)\right) .
$$


The $(S V)$-stratum: This is the set of $(u, v)$ where the distance squared function of $\tilde{P}$ has an $A_{4}$-singularity. If $D$ denotes the distance squared function on $\tilde{P}$ then we have

$$
\begin{aligned}
j^{1} D^{\prime} & =-x_{1} v, \\
j^{1} D^{\prime \prime} & =-2 x_{2} v-4 a, \\
j^{1} D^{\prime \prime \prime} & =-12 u x_{1}-6 x_{3} v, \\
j^{1} D^{(4)} & =-48 u x_{2}-24 x_{4} v-48 b-120 t .
\end{aligned}
$$

Hence, using the implicit function theorem, we can write $a, b, u$ and $v$ as functions of $t$ as follow:

$$
\begin{aligned}
& a=a_{1} t+a_{2} t^{2}+O\left(t^{3}\right), \\
& b=b_{1} t+b_{2} t^{2}+O\left(t^{3}\right), \\
& u=u_{1} t+u_{2} t^{2}+O\left(t^{3}\right), \\
& v=v_{1} t+v_{2} t^{2}+O\left(t^{3}\right) .
\end{aligned}
$$

To obtain all the coefficients $a_{i}, b_{i}, u_{i}$ and $v_{i}$ we consider the system of equations $D^{(i)}(t)=0$ for $i=1,2,3,4$, jet by jet.

The coefficient matrix of (4.15) is

$\left[\begin{array}{c|cccc}t & a & b & u & v \\ \hline 0 & 0 & 0 & 0 & -x_{1} \\ 0 & -4 & 0 & 0 & -2 x_{2} \\ 0 & 0 & 0 & -12 x_{1} & 6 x_{3} \\ 120 & 0 & -48 & -48 x_{2} & 24 x_{4}\end{array}\right]$,

which has rank 4 if and only if $x_{1} \neq 0$. Therefore we have

$$
a_{1}=0, b_{1}=\frac{-5}{2}, u_{1}=0 \text { and } v_{1}=0 .
$$

Next, we have

$$
\begin{aligned}
j^{2} D^{\prime}(t) & =-v_{2} x_{1} t^{2}=0 \Rightarrow v_{2}=0, \\
j^{2} D^{\prime \prime}(t) & =-4 a_{2} t^{2}=0 \Rightarrow a_{2}=0, \\
j^{2} D^{\prime \prime \prime}(t) & =\left(-12 x_{1} u_{2}+60\right) t^{2}=0 \Rightarrow u_{2}=\frac{5}{x_{1}}, \\
j^{2} D^{(4)}(t) & =\left(\frac{-240 x_{2}}{x_{1}}+600-360 z_{6}-48 b_{2}\right) t^{2}=0 \Rightarrow b_{2}=-\frac{5}{2} \frac{\left(3 x_{1} z_{6}-5 x_{1}+2 x_{2}\right)}{x_{1}} .
\end{aligned}
$$

Subsequently, for jet 3 we get

$$
\begin{aligned}
j^{3} D^{\prime}(t) & =-v_{3} x_{1} t^{3}=0 \Rightarrow v_{3}=0 \\
j^{3} D^{\prime \prime}(t) & =\left(-4 a_{3}-20\right) t^{3}=0 \Rightarrow a_{3}=-5 \\
j^{3} D^{\prime \prime \prime}(t) & \left.=-12 u_{3} x_{1}+\frac{120\left(3 x_{1} z_{6}-5 x_{1}+2 x_{2}\right)}{x_{1}}+300-\frac{240 x_{2}}{x_{1}}-120 z_{6}\right) t^{3}=0 \Rightarrow u_{3}=\frac{5\left(4 z_{6}-5\right)}{x_{1}}, \\
j^{3} D^{(4)}(t) & =\left(\frac{-1200 x_{3}}{x_{1}}-840 z_{7}+1800 z_{6}+\frac{600\left(3 x_{1} z_{6}-5 x_{1}+2 x_{2}\right)}{x_{1}}-\frac{240\left(4 z_{6}-5\right) x_{2}}{x_{1}}-48 b 3\right) t^{3}=0 \\
\Rightarrow & b_{3}=\frac{5}{2} \frac{\left(30 x_{1} z_{6}-7 x_{1} z_{7}-8 x_{2} z_{6}-25 x_{1}+20 x_{2}-10 x_{3}\right)}{x_{1}} .
\end{aligned}
$$


Similarly, we can obtain other coefficients. In particular $v_{4}=\frac{5}{x_{1}}$ and $v_{5}=\frac{5\left(4 z_{6}-5\right)}{x_{1}}$.

Therefore the $(V T)$-stratum is given by

$$
(u, v)=\left(\frac{5}{x_{1}} t^{2}+u_{3} t^{3}+O\left(t^{4}\right), \frac{5}{x_{1}} t^{4}+v_{5} t^{5}+O\left(t^{6}\right)\right),
$$

which is $\mathcal{A}$-equivalent to

$$
\left(t^{2}+O\left(t^{3}\right), \frac{x_{1}}{5}\left(v_{5}-2 u_{3}\right) t^{5}+O\left(t^{6}\right)\right)
$$

by using the change of coordinate $(u, v) \mapsto\left(u, v-u^{2}\right)$ on target. This is a ramphoid cusp singularity at origin if and only if $\frac{x_{1}}{5}\left(v_{5}-2 u_{3}\right)=\frac{-16}{x_{1}} z_{6} \neq 0$.

The $(T C)$-stratum: This consists of points $(u, v)$ such that $\tilde{P}_{(u, v)}(t)$ has a multilocal singularity for which two pieces of the curve have an ordinary tangency at a given point. We know from [21] that this stratum is diffeomorphic to half a line. To find its parametrization, suppose that $h(t, w)=\langle\gamma(t), w\rangle$ be the height function along the unit vector $w$. The height function has two singularities at the same level, that is

$$
h(t, w)-d=\left(t-t_{1}\right)^{2}\left(t-t_{2}\right)^{2} \tilde{h}(t, w),
$$

for some smooth function $\tilde{h}(t, w)$.

The direction of the projection is along the vector $\gamma\left(t_{2}\right)-\gamma\left(t_{1}\right)$ and the $(T C)$-stratum in the $(u, v)$ plane is given by

$$
\begin{aligned}
& u=\frac{\gamma_{2}\left(t_{2}\right)-\gamma_{2}\left(t_{1}\right)}{\gamma_{1}\left(t_{2}\right)-\gamma_{1}\left(t_{1}\right)}, \\
& v=\frac{\gamma_{3}\left(t_{2}\right)-\gamma_{3}\left(t_{1}\right)}{\gamma_{1}\left(t_{2}\right)-\gamma_{1}\left(t_{1}\right)} .
\end{aligned}
$$

We equate the coefficients of the 5-jet of $h(t, w)-d$ and $\left(t-t_{1}\right)^{2}\left(t-t_{2}\right)^{2} \tilde{h}(t, w)$ as functions in $t$. Therefore, we get $t_{2}=-t_{1}+O\left(t_{1}^{2}\right)$. Subsequently, performing the same method we get the higher terms for $t_{2}$ that is $t_{2}=-t_{1}-t_{1}^{2}+O\left(t_{1}^{3}\right)$.

Consequently, using (4.16), the $(T C)$-stratum is given by the following parametrization:

$$
(u, v)=\left(\frac{-1}{x_{1}} t^{2}+\text { h.o.t, } \frac{1}{x_{1}} t^{4}+\text { h.o.t }\right) .
$$

The $(S I)$-stratum: This happens when the height function on $\tilde{P}_{(u, v)}(t)$ has a higher order inflection at some point $t$ (equivalently, height function has an $A_{3}$-singularity). By an straightforward computation on curvature and its first derivative one can obtain $v=0$ as the desire stratum.

One can observe that each stratum in the bifurcation of $\tilde{P}$ of a ramphoid cusp singularity is homeomorph to corresponding stratum in Theorem 3.6. According to Definition of $F R S$ equivalent in Chapter $\S 3$, to show that two families are $F R S$-equivalent we should check the number and position of inflections and vertices in each stratum as well. 
At the cusp stratum we have

$$
\kappa_{(u, v)}(t)=\frac{-2 x_{1} v-12 x_{1} u t^{2}+6 x_{3} v t^{2}+16 t^{3}+f(t, u, v)}{\left(\left(2 t-u x_{1}+\text { h.o.t }\right)^{2}+\left(4 t^{3}+5 t^{4}+v x_{1}+\text { h.o.t }\right)^{2}\right)^{3 / 2}},
$$

and

$$
\kappa_{(u, v)}^{\prime}(t)=\frac{-12 u v x_{1}^{2}+12 x_{1}^{2} x_{2} v\left(u^{2}+v^{2}\right)+24 x_{1} v\left(1-2 x_{2} u\right) t+\cdots+120 t^{5}+g(t, u, v)}{\left(\left(2 t-u x_{1}+\text { h.o.t }\right)^{2}+\left(4 t^{3}+5 t^{4}+v x_{1}+\text { h.o.t }\right)^{2}\right)^{5 / 2}},
$$

where $f(t, u, v) \in \mathcal{M}_{3,1}^{4}$ and $g(t, u, v) \in \mathcal{M}_{3,1}^{6}$.

Now using the equation of the cusp stratum together with the fact that there are 2 inflections and 3 vertices concentrated at the cusp point, one can conclude that there exist one inflection and 2 vertices in this stratum when $u>0$ and just one inflection when $u<0$. (See (2) and (14) in Figure 4.7.)

For the other strata in (4.7), it is now a matter of going around the origin in the parameter space and plotting the inflections and vertices on the curve as we cross the various strata of the bifurcations set.

Consequently, the family $\tilde{P}$ is an FRS-generic family of a ramphoid cusp and is $F R S$ equivalent to the model $\left(t^{2}, t^{4}+t^{5}+t^{6}+u t^{3}+v t\right)$. The bifurcation of the ramphoid cusp $\tilde{P}(t, 0,0)$ is given in Figure 4.7 . 


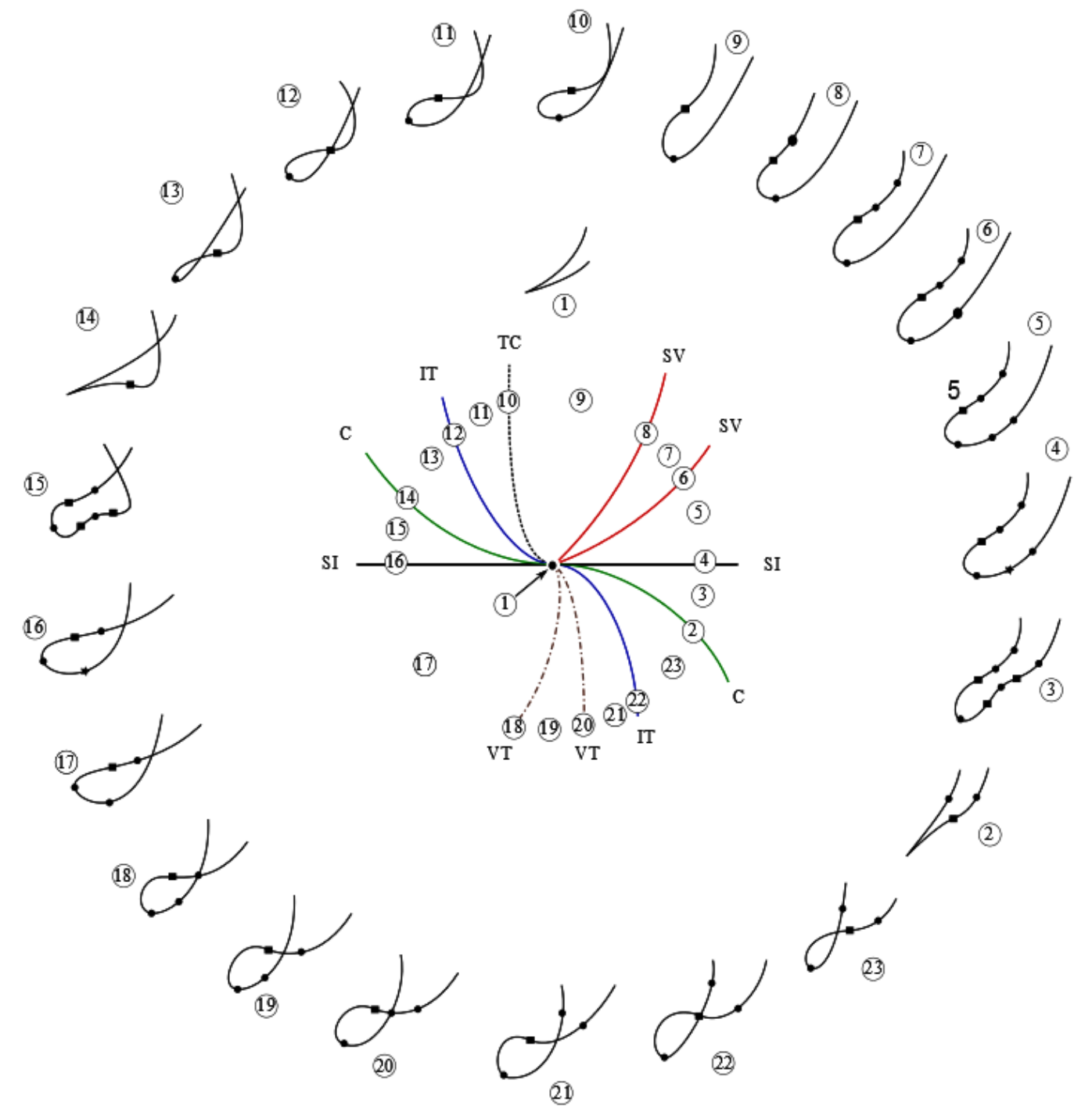

Figure 4.7 - Bifurcation of the ramphoid cusp. The dots represent the points of vertices and the squares represent the points of inflections. 


\section{THE GEOMETRY OF ORTHOGONAL PROJECTION OF SURFACES IN $\mathbb{R}^{3}$}

The geometry of surfaces is a subject that fascinated many mathematicians and users of mathematics. One way to study geometry of surfaces is to compare the surfaces in $\mathbb{R}^{3}$ with model submanifolds.

In this chapter we deal with the contact of regular surface $M$ with parallel lines. As seen in $\S 2.5 .3$ this contact measured by singularities of family of orthogonal projections. For surfaces in $\mathbb{R}^{3}$, given a point $p \in M$, we choose a local parametrisation $\phi: U \subset \mathbb{R}^{2} \rightarrow M$ of $M$ at $p$ with $\phi(0)=p$. In Table 2.2 can be found the local $\mathcal{A}$-codimension $\leq 2$ singularities of an orthogonal projection of $M$. The image of the singular set of an orthogonal projection of the surface is called profile or apparent contour. The profile is a plane curve and may have singularities. The changing in the profile carry a great deal of information about the surface. The aim of this chapter is to extend the FRS-theory for discriminants of map germs from $\mathbb{R}^{2}$ to $\mathbb{R}^{2}$. We do this by considering the geometry of the profile of an orthogonal projection. For instance: How many inflections, cusps and vertices do the profiles have? When do they have inflections, cusps and vertices?

After a brief preliminaries in $\S 5.1$, we consider in $\$ 5.2$ the fold singularity. In Theorems 5.2, 5.3 we investigate the geometric conditions for surface $M$ when the profile has vertex or inflection at the point $p$. In $\S 5.3$ we study cusp singularity. Fold and cusp singularities are $\mathcal{A}_{e}$-stable. The $\mathcal{A}_{e}$-codimension 1 singularities of an orthogonal projection called swallowtail and lips/beaks. We study these singularities and geometry of their profiles in $§ 5.4$ and $\S 5.5$ respectively.

We bring up the $\mathcal{A}_{e}$-codimension 2 singularities of an orthogonal projection of the surface $M$ which are called butterfly, goose and gulls, in §5.6, §5.7 and 5.8 respectively. Theorems 5.7 and 5.8 are devoted to the profile at a butterfly singularity and a goose singularity respectively. 
One important geometric phenomena in the study of the profile is the second order vertex point. To obtain theses points we consider the contact of surface $M$ with cylinder. In $\S 5.8 .1$ we consider the contact of a smooth surface $M$ with circular cylinder at the cusp of Gauss point and obtain a new curve called $A_{4}$-curve. This curve is a robust feature and consists of locus of all points on surface which the profile has a second order vertex.

Finally in $\$ 5.9$ we extend $F R S$-theory for discriminant of map germs from $\mathbb{R}^{2}$ to $\mathbb{R}^{2}$.

\subsection{Preliminaries}

In this part we give some basic concepts in the study of surfaces in $\mathbb{R}^{3}$.

\subsubsection{The Monge form parametrization}

As seen in $\S 2.5 .3$ the family of orthogonal projections $P: U \times S^{2} \rightarrow T S^{2}$ on $M$ is given by

$$
P(x, y, w)=(w, \phi(x, y)-\langle\phi(x, y), w\rangle w) .
$$

We denote the second component of $P$ by $P_{w}$ and consider $P_{w}$ as the orthogonal projection of surface $M$ along the fixed direction $w$. In Table 2.2 we summarized the local $\mathcal{A}$-singularities of $P_{w}$.

At each point $p$ on the surface $M$, we can choose a coordinate system in $\mathbb{R}^{3}$ such that $p$ is the origin, the plane $y=0$ is the projection plane and the surface $M$ is locally the graph of some function $z=f(x, y)$, with $(x, y)$ in an open subset $U$ of $\mathbb{R}^{2}$ containing origin. Then we have the Monge form parametrisation $\phi(x, y)=(x, y, f(x, y)),(x, y) \in U$, of $M$ at $p$. Note that the Taylor expansion of $f$ at the origin has no constant or linear terms. We write

$$
\begin{aligned}
f(x, y)= & a_{20} x^{2}+a_{21} x y+a_{22} y^{2}+a_{30} x^{3}+a_{31} x^{2} y+a_{32} x y^{2}+a_{33} y^{3} \\
& +a_{40} x^{4}+a_{41} x^{3} y+a_{42} x^{2} y^{2}+a_{43} x y^{3}+a_{44} y^{4}+O(5) .
\end{aligned}
$$

Let a surface $M$ be given in Monge form

$$
\phi(x, y)=(x, y, f(x, y))=x e_{1}+y e_{2}+f(x, y) e_{3}
$$

with $f$ as in (5.1), where

$$
e_{1}=(1,0,0), \quad e_{2}=(0,1,0), \quad e_{3}=(0,0,1)
$$

Consider the Monge form at $q=\left(x_{0}, y_{0}, f\left(x_{0}, y_{0}\right)\right)$. Firstly, we move $q$ to the origin via translation, and we obtain

$$
\begin{aligned}
\tilde{\phi}(x, y) & =\left(x, y,-f\left(x_{0}, y_{0}\right)+f\left(x+x_{0}, y+y_{0}\right)\right) \\
& =x e_{1}+y e_{2}+\left(-f\left(x_{0}, y_{0}\right)+f\left(x+x_{0}, y+y_{0}\right)\right) e_{3} .
\end{aligned}
$$


We define an orthonormal frame at $q$ by

$$
\begin{aligned}
& \tilde{e}_{1}=\frac{\left(1,0, f_{x}\left(x_{0}, y_{0}\right)\right)}{\sqrt{1+f_{x}\left(x_{0}, y_{0}\right)^{2}}}, \\
& \tilde{e}_{2}=\frac{\left(-f_{x}\left(x_{0}, y_{0}\right) f_{y}\left(x_{0}, y_{0}\right), 1+f_{x}\left(x_{0}, y_{0}\right)^{2}, f_{y}\left(x_{0}, y_{0}\right)\right)}{\sqrt{1+f_{x}\left(x_{0}, y_{0}\right)^{2}} \sqrt{1+f_{x}\left(x_{0}, y_{0}\right)^{2}+f_{y}\left(x_{0}, y_{0}\right)^{2}}}, \\
& \tilde{e}_{3}=\frac{\left(-f_{x}\left(x_{0}, y_{0}\right),-f_{y}\left(x_{0}, y_{0}\right), 1\right)}{\sqrt{1+f_{x}\left(x_{0}, y_{0}\right)^{2}+f_{y}\left(x_{0}, y_{0}\right)^{2}}} .
\end{aligned}
$$

We obtain the change of basis matrix

$$
B=\left(\begin{array}{cccc}
\frac{1}{\sqrt{1+f_{x}\left(x_{0}, y_{0}\right)^{2}}} & \frac{-f_{x}\left(x_{0}, y_{0}\right) f_{y}\left(x_{0}, y_{0}\right)}{\sqrt{1+f_{x}\left(x_{0}, y_{0}\right)^{2}} \sqrt{1+f_{x}\left(x_{0}, y_{0}\right)^{2}+f_{y}\left(x_{0}, y_{0}\right)^{2}}} & -\frac{f_{x}\left(x_{0}, y_{0}\right)}{\sqrt{1+f_{x}\left(x_{0}, y_{0}\right)^{2}+f_{y}\left(x_{0}, y_{0}\right)^{2}}} \\
0 & \frac{1+f_{x}\left(x_{0}, y_{0}\right)^{2}}{\sqrt{1+f_{x}\left(x_{0}, y_{0}\right)^{2}} \sqrt{1+f_{x}\left(x_{0}, y_{0}\right)^{2}+f_{y}\left(x_{0}, y_{0}\right)^{2}}} & -\frac{f_{y}\left(x_{0}, y_{0}\right)}{\sqrt{1+f_{x}\left(x_{0}, y_{0}\right)^{2}+f_{y}\left(x_{0}, y_{0}\right)^{2}}} \\
\frac{f_{x}\left(x_{0}, y_{0}\right)}{\sqrt{1+f_{x}\left(x_{0}, y_{0}\right)^{2}}} & \frac{f_{y}\left(x_{0}, y_{0}\right)}{\sqrt{1+f_{x}\left(x_{0}, y_{0}\right)^{2}} \sqrt{1+f_{x}\left(x_{0}, y_{0}\right)^{2}+f_{y}\left(x_{0}, y_{0}\right)^{2}}} & \frac{1}{\sqrt{1+f_{x}\left(x_{0}, y_{0}\right)^{2}+f_{y}\left(x_{0}, y_{0}\right)^{2}}}
\end{array}\right),
$$

therefore we have

Substituting (5.5) into (5.2), we obtain

$$
\left(\begin{array}{l}
e_{1} \\
e_{2} \\
e_{3}
\end{array}\right)=B\left(\begin{array}{c}
\tilde{e}_{1} \\
\tilde{e}_{2} \\
\tilde{e}_{3}
\end{array}\right)
$$

$$
\tilde{\phi}(x, y)=a\left(x, y, x_{0}, y_{0}\right) \tilde{e}_{1}+b\left(x, y, x_{0}, y_{0}\right) \tilde{e}_{2}+c\left(x, y, x_{0}, y_{0}\right) \tilde{e}_{3},
$$

where

$$
\begin{aligned}
a\left(x, y, x_{0}, y_{0}\right) & =\frac{\left(-f\left(x_{0}, y_{0}\right)+f\left(x+x_{0}, y+y_{0}\right)\right) f_{x}\left(x_{0}, y_{0}\right)+x}{\sqrt{1+f_{x}\left(x_{0}, y_{0}\right)^{2}}}, \\
b\left(x, y, x_{0}, y_{0}\right) & =\frac{\left(-f\left(x_{0}, y_{0}\right)+f\left(x+x_{0}, y+y_{0}\right)\right) f_{y}\left(x_{0}, y_{0}\right)-f_{x}\left(x_{0}, y_{0}\right) f_{y}\left(x_{0}, y_{0}\right) x+\left(1+f_{x}\left(x_{0}, y_{0}\right)^{2}\right) y}{\sqrt{1+f_{x}\left(x_{0}, y_{0}\right)^{2}} \sqrt{1+f_{x}\left(x_{0}, y_{0}\right)^{2}+f_{y}\left(x_{0}, y_{0}\right)^{2}}}, \\
c\left(x, y, x_{0}, y_{0}\right) & =\frac{-f\left(x_{0}, y_{0}\right)+f\left(x+x_{0}, y+y_{0}\right)-f_{x}\left(x_{0}, y_{0}\right) x-f_{y}\left(x_{0}, y_{0}\right) y}{\sqrt{1+f_{x}\left(x_{0}, y_{0}\right)^{2}+f_{y}\left(x_{0}, y_{0}\right)^{2}}} .
\end{aligned}
$$

Set $x(s, t)$ and $y(s, t)$ such that $a\left(x(s, t), y(s, t), x_{0}, y_{0}\right)=s$ and $b\left(x(s, t), y(s, t), x_{0}, y_{0}\right)=t$. Write $\tilde{\phi}(s, t)=\tilde{\phi}(x(s, t), y(s, t))$ and replace $(s, t)$ for $(x, y)$. We obtain the Monge form at $\left(x_{0}, y_{0}, f\left(x_{0}, y_{0}\right)\right)$ :

$$
\tilde{\phi}(x, y)=\left(x, y, g\left(x, y, x_{0}, y_{0}\right)\right)=x \tilde{e}_{1}+y \tilde{e}_{2}+g\left(x, y, x_{0}, y_{0}\right) \tilde{e}_{3},
$$

where $g\left(x, y, x_{0}, y_{0}\right)$ is given by

$$
\begin{aligned}
g\left(x, y, x_{0}, y_{0}\right)= & A_{20}\left(x_{0}, y_{0}\right) x^{2}+A_{21}\left(x_{0}, y_{0}\right) x y+A_{22}\left(x_{0}, y_{0}\right) y^{2} \\
& +A_{30}\left(x_{0}, y_{0}\right) x^{3}+A_{31}\left(x_{0}, y_{0}\right) x^{2} y+A_{32}\left(x_{0}, y_{0}\right) x y^{2}+A_{33}\left(x_{0}, y_{0}\right) y^{3}+O(x, y)^{4}
\end{aligned}
$$

with

$$
\begin{aligned}
& A_{20}\left(x_{0}, y_{0}\right)=a_{20}+3 a_{30} x_{0}+a_{32} y_{0}+O(2), \\
& A_{21}\left(x_{0}, y_{0}\right)=a_{21}+2 a_{31} x_{0}+2 a_{32} y_{0}+O(2), \\
& A_{22}\left(x_{0}, y_{0}\right)=a_{22}+a_{32} x_{0}+3 a_{33} y_{0}+O(2) .
\end{aligned}
$$




\subsubsection{The modified family of orthogonal projections}

If $w \in T_{p} M$, the $P_{w}$ is locally a diffeomorphism. Suppose that $w_{0} \in T_{p} M$. We can rotate the coordinate axes if necessary and set $w_{0}=(0,1,0)$. With the above settings, we have

$$
P_{w_{0}}(x, y)=(x, f(x, y))
$$

When considering the family of orthogonal projections, as we are not allowed to apply diffeomorphisms, the computations simplify if we use spherical coordinates. We parametrise the directions near $w_{0}=(0,1,0)$ by $w=(\sin (v) \cos (u), \sin (v) \sin (u), \cos (v))$, with $u$ and $v$ close to $\pi / 2$, and rotate $w$ to the vector $w_{0}=(0,1,0)$ to have the projection to the fix plane $y=0$. To be more precise, the rotation matrix is

$$
R=\left[\begin{array}{ccc}
\sin (u) & -\cos (u) & 0 \\
\sin (v) \cos (u) & \sin (v) \sin (u) & \cos (v) \\
-\cos (u) \cos (v) & -\cos (v) \sin (u) & \sin (v)
\end{array}\right]
$$

The projection of the point $(x, y, f(x, y))$ after performing the rotation (5.7) and the changes of coordinates $u \mapsto u+\pi / 2$ and $v \mapsto v+\pi / 2$ becomes

$$
\tilde{P}(x, y, u, v)=\left[\begin{array}{c}
\cos (u) x+\sin (u) y \\
0 \\
\cos (u) \sin (v) y-\sin (u) \sin (v) x+\cos (v) f(x, y)
\end{array}\right]
$$

In fact, $\tilde{P}$ is a map from $M \times S^{2}$ to $\mathbb{R}^{2}$ and we call it the modified family of orthogonal projections. One can observe from rotation carried out above that the family $P$ of orthogonal projections on $M$ is $\mathcal{A}$-equivalent to the modified family of projections $\tilde{P}$.

The set of critical points of $\tilde{P}$ is denoted by $\Sigma\left(\tilde{P}_{w}\right)$. This set is called the contour generator of $M$ and is given by

$$
\left\{(x, y) \in \mathbb{R}^{2} \mid \text { determinant of Jacobian of } \tilde{P}_{w}=0\right\},
$$

where $\tilde{P}_{w}(x, y)=\tilde{P}(x, y, u, v)$ is as in (5.8). The image of $\Sigma\left(\tilde{P}_{w}\right)$ by $\tilde{P}$ is called the apparent contour or profile of $M$ along the direction $w$.

Proposition 5.1 (Proposition 6.11 of [24]). (i) The contour generator is a singular curve at $p$ if and only if $p$ is a parabolic point and $w$ is the unique asymptotic direction at $p$.

(ii) The profile is a smooth curve at $\tilde{P}_{w}(p)$ if and only if $w$ is not an asymptotic direction at $p$.

Theorem 5.1 (Koenderink's Theorem). Suppose that the apparent contour is a smooth curve at $\tilde{P}_{w}(p)$. Then the Gaussian curvature of $M$ at $p$ is equal to the product of the curvature of the apparent contour together with the curvature of the normal section of $M$ at $p$ along the direction $w$. 
Next definition devoted to some geometric concepts which we use during this chapter.

Definition 5.1. (i) A point $p$ in the surface $M$ is called a flecnodal point if there exists a tangent line through $p$ which has at least 4-point contact with $M$.

(ii) If $\alpha:(-\epsilon, \epsilon) \rightarrow M$ be a smooth curve on $M$ then $\kappa_{g}=\left\langle\alpha^{\prime \prime}, t \times N\right\rangle$ is called geodesic curvature. A point $p$ on $\alpha$ is a geodesic inflection if $\kappa_{g}=0$ at $p$.

\subsection{Fold}

An algebraic condition to have fold singularity is $a_{22} \neq 0$ (Theorem 6.7 of [24]). The geometric characterisation of a fold singularity of $P_{w_{0}}$ at the point $p$ is that the direction of projection $w$ is tangent to $M$ at $p$ but not asymptotic at $p$.

The fold singularity is $\mathcal{A}_{e}$-stable so we consider $\tilde{P}_{w_{0}}$, with $w_{0}=(0,1,0)$. Applying change of coordinate $y \mapsto y-\frac{a_{21}}{2 a_{22}} x$ in (5.1) we obtain

$$
\begin{aligned}
& j^{4} f(x, y)=\left(-(1 / 4) a_{21}^{2} / a_{22}+a_{20}\right) x^{2}+a_{22} y^{2}+\left((1 / 4) a_{32} a_{21}^{2} / a_{22}^{2}-\right. \\
&\left.(1 / 2) a_{31} a_{21} / a_{22}+a_{30}-(1 / 8) a_{33} a_{21}^{3} / a_{22}^{3}\right) x^{3}+ \\
&\left(-a_{32} a_{21} / a_{22}+a_{31}+(3 / 4) a_{33} a_{21}^{2} / a_{22}^{2}\right) y x^{2}+ \\
&\left(a_{32}-(3 / 2) a_{33} a_{21} / a_{22}\right) y^{2} x+a_{33} y^{3}+\left((1 / 4) a_{42} a_{11}^{2} / a_{22}^{2}-\right. \\
&\left.(1 / 8) a_{43} a_{21}^{3} / a_{22}^{3}-(1 / 2) a_{41} a_{21} / a_{22}+a_{40}+(1 / 16) a_{44} a_{21}^{4} / a_{22}^{4}\right) x^{4}+ \\
&\left(a_{41}-(1 / 2) a_{44} a_{21}^{3} / a_{22}^{3}-a_{42} a_{21} / a_{22}+(3 / 4) a_{43} a_{21}^{2} / a_{22}^{2}\right) y x^{3}+ \\
&\left((3 / 2) a_{44} a_{21}^{2} / a_{22}^{2}+a_{42}-(3 / 2) a_{43} a_{21} / a_{22}\right) y^{2} x^{2}+ \\
&\left(-2 a_{44} a_{21} / a_{22}+a_{43}\right) y^{3} x+a_{44} y^{4} .
\end{aligned}
$$

The contour generator is given by $2 a_{22} y+O(2)=0$. As $a_{22} \neq 0$, the implicit function theorem guarantees that one can write $y$ locally as function of $x$ such that $y=b_{2} x^{2}+O\left(x^{3}\right)$. We find that

$$
b_{2}=-\frac{\left(4 a_{22}^{2} a_{31}-4 a_{22} a_{21} a_{32}+3 a_{33} a_{21}^{2}\right)}{8 a_{22}^{3}} .
$$

Substituting $y=b_{2} x^{2}+O\left(x^{3}\right)$ in $\tilde{P}_{w}(x, y)$, we obtain the expression of the profile which is a regular plane curve $(x, Y(x))$ with

$$
Y(x)=\frac{4 a_{20} a_{22}-a_{21}^{2}}{4 a_{22}} x^{2}+\frac{8 a_{22}^{3} a_{30}-4 a_{21} a_{22}^{2} a_{31}+2 a_{21}^{2} a_{22} a_{32}-a_{21}^{3} a_{33}}{8 a_{22}^{3}} x^{3}+O\left(x^{4}\right) .
$$


If $\kappa$ denotes the curvature of profile then according to (5.11) we have

$$
\begin{aligned}
\kappa_{c}=\kappa(0) & =\frac{4 a_{20} a_{22}-a_{21}^{2}}{4 a_{22}}, \\
\kappa^{\prime}(0) & =\frac{8 a_{22}^{3} a_{30}-4 a_{21} a_{22}^{2} a_{31}+2 a_{21}^{2} a_{22} a_{32}-a_{21}^{3} a_{33}}{8 a_{22}^{3}} .
\end{aligned}
$$

Theorem 5.2 ([17]). For a given surface $M$ in $\mathbb{R}^{3}$ let the projection map $P_{w}(x, y)=(x, f(x, y))$ has a singularity of type fold at the origin. Then

(i) The profile has an ordinary vertex at $P_{w}(0,0)$ if and only if the origin is not a parabolic point and $M$ has an $A_{3}$-contact with $C_{w, \lambda}$ at the origin, where $C_{w, \lambda}$ denotes a cylinder which contains the origin and whose axis is parallel to $w$ and contains a point $(0,0, \lambda)=$ $\left(0,0,1 / \kappa_{c}\right)$ such that $\kappa_{c}$ is the curvature of the profile at $P_{w}(0,0)$.

(ii) The profile has a second order vertex at $P_{w}(0,0)$ if and only if the origin is not a parabolic point and $M$ has an $A_{4}$-contact with $C_{w, \lambda}$ at the origin.

(iii) The profile has a third order vertex at $P_{w}(0,0)$ if and only if the origin is not a parabolic point and $M$ has an $A_{5}$-contact with cylinder $C_{w, \lambda}$ at the origin.

Remark 5.1. According to Theorem 5.2, the profile has an ordinary vertex if and only if the point $p$ is not parabolic and the cubic polynomial $C_{3}(x, y)=a_{30} x^{3}+a_{31} x^{2} y+a_{32} x y^{2}+a_{33} y^{3}$ has the root $\left(2 a_{22},-a_{21}\right)$. On the other hand, we know from definition of conjugate direction that if $w=a \partial_{x}+b \partial_{y}$ then the conjugate of $w$ is $\bar{w}=(a m+b n) \partial_{x}-(a l+b m) \partial_{y}$ where $l, m$ and $n$ are the coefficients of the second fundamental form of $M$. As $w=(0,1,0)$ so $a=0$ and $b=1$ thus $a m+b n=n=\frac{f_{y y}}{\sqrt{1+f_{x}^{2}+f_{y}^{2}}}=2 a_{22}$ and $-(a l+b m)=-m=-\frac{f_{x y}}{\sqrt{1+f_{x}^{2}+f_{y}^{2}}}=-a_{21}$ therefore $C_{3}(\bar{w})=0$.

Next theorem gives information about the geometry of profile when it has an inflection.

Theorem 5.3. For a given surface $M$ in $\mathbb{R}^{3}$ let the projection map $P_{w}(x, y)=(x, f(x, y))$ has a singularity of type fold at the origin. Then generically

(i) The profile has an ordinary inflection at $P_{w}(0,0)$ if and only if the point $p$ is parabolic, the direction of projection is not-asymptotic.

(ii) The profile has a second order inflection at $P_{w}(0,0)$ if and only if the point $p$ is Cusp of Gauss and $w$ is not an asymptotic direction.

Proof. We take $M$ locally in the Monge form $\phi(x, y)=(x, y, f(x, y))$ with $f$ as in (5.1). According to (5.12) one can see that the profile has an ordinary inflection if and only if $4 a_{22} a_{20}-$ $a_{21}^{2}=0$ i.e. the point $p$ is parabolic point. As seen in $\$ 2.5 .1$, the contact of surface $M$ with its tangent plane captured by singularities of height function along the normal direction $N_{0}=$ 
$(0,0,1)$ at $p$ which is given by $f(x, y)$. Putting $4 a_{22} a_{20}-a_{21}^{2}=0$ in (5.10) we get the height function has $A_{\geq 2}$-singularity at $(0,0)$.

A second order inflection happens when $\kappa_{c}=\kappa_{c}^{\prime}=0$. Equivalently $\left(4 a_{22} a_{20}-a_{21}^{2}\right)=0$ and $\left(a_{21}^{3} a_{33}-2 a_{21}^{2} a_{22} a_{32}+4 a_{21} a_{22}^{2} a_{31}-8 a_{22}^{3} a_{30}\right)=0$. Substituting these in the (5.10) we get the height function has $A_{\geq 3}$-singularity, this means that $p$ is cusp of Gauss (see Figure 5.1 for evolute at a second inflection point).

Remark 5.2. Generically, the profile at a fold singularity does not have a third order inflection.

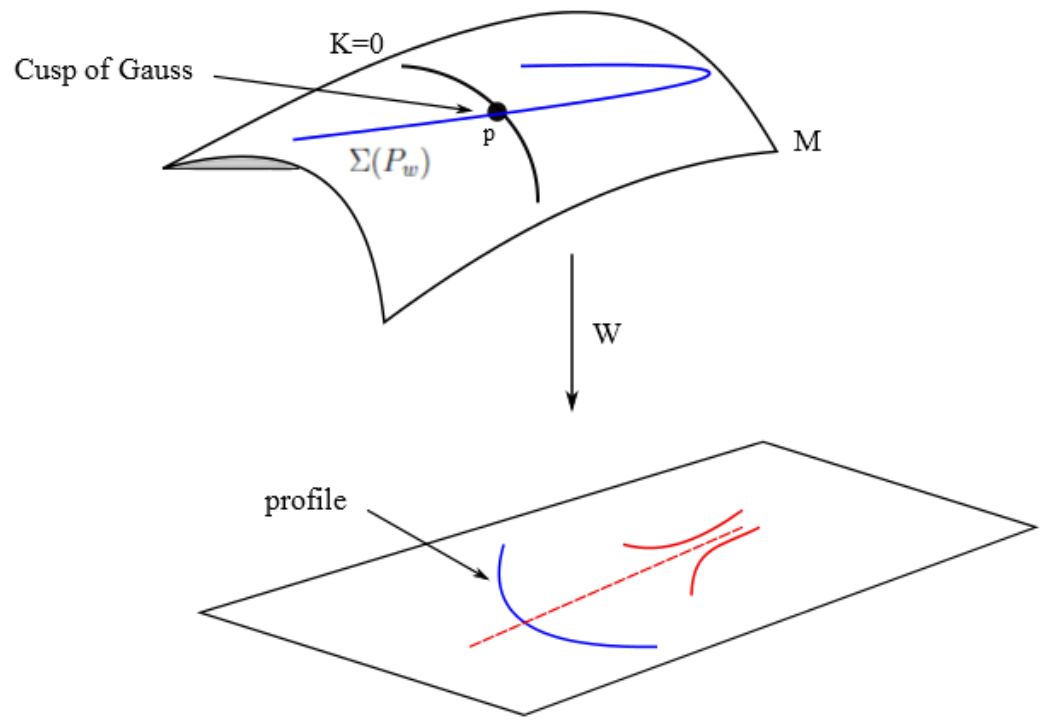

Figure 5.1 - The profile has second order inflection if critical set passes through the cusp of Gauss point. The red curve indicates the evolute at a second order inflection point.

\subsection{Cusp}

Suppose that $P_{w}(x, y)$ has singularity of type cusp at $p=\phi(0,0)$, i.e. $a_{22}=0$ and $a_{21} a_{33} \neq 0$. The geometric characterization for the cusp singularity is that the point $p$ is a hyperbolic point and the direction of projection is an asymptotic direction (see chapter 6 of [24] for precise details).

Recall that the cusp singularity is $\mathcal{A}_{e}$-stable. The singular set $\Sigma\left(\tilde{P}_{w}\right)$ is given by $a_{21} x+O(2)=$ 0 . As $a_{21} \neq 0$, by the implicit function theorem, we can write locally $x$ as a function of $y$ with

$$
x=x(y)=-\frac{3 a_{33}}{a_{21}} y^{2}+\frac{2\left(3 a_{33} a_{32}-2 a_{44} a_{21}\right)}{a_{21}^{2}} y^{3}+O\left(y^{4}\right) .
$$

Substituting in $\tilde{P}_{w}(x, y)$ we get the parametrisation of the profile given by

$$
(X(y), Y(y))=\left(-\frac{3 a_{33}}{a_{21}} y^{2}+O\left(y^{3}\right),-2 a_{33} y^{3}+O\left(y^{4}\right)\right)
$$


witch has a cusp singularity.

According to discussion in $\S 3.2$ we know that there is an accumulation of 3 vertices and 2 inflections concentrated at the cusp point. Also from Theorem 3.4 the full evolute of a cusp is a smooth curve which has an ordinary tangency at the cusp point together with its limiting normal line at the cusp point counted twice. The limiting normal line to the profile at the cusp point separates the profile and its proper evolute.

\subsection{Swallowtail}

The algebraic conditions for swallowtail singularity are

$$
a_{22}=0, a_{33}=0, a_{21} \neq 0 \text { and } a_{44} \neq 0
$$

The following observation gives us information about versality of the family of modified orthogonal projections (5.8) at swallowtail singularity.

Proposition 5.2. Let $F(x, y, u)=\tilde{P}(x, y, u, 0)$ be a 1-parameter family of a swallowtail singularity $\tilde{P}(x, y, 0,0) . F$ is an $\mathcal{A}_{e}$-versal family of a swallowtail singularity.

Proof. By the change of coordinate $x \mapsto x-u y$ in the source, we get the family $F(x, y, u)=$ $\tilde{P}(x, y, u, 0)=(x, f(x+u y, y))$. The swallowtail is 4 - $\mathcal{A}$-determined, so we need to show that

$$
L \mathcal{A}_{e} \cdot \tilde{F}_{0}+\mathbb{R} .\{\dot{F}\}=\mathcal{E}(2,2)
$$

holds in the 4-jet space. We have

$$
\begin{aligned}
\dot{F}=\frac{\partial F}{\partial u}(x, y, 0)=\left(0, y f_{x}(x, y)\right) & \\
j^{3}\left(\frac{\partial F_{0}}{\partial x}\right)=\left(1,2 a_{02}+a_{21} y+3 a_{30} x^{2}+2 a_{31} x y+a_{32} y^{2}\right. & \left.\quad+4 a_{40} x^{3}+3 a_{41} x^{2} y+22 a_{42} x y^{2}+a_{43} y^{3}\right), \\
j^{3}\left(\frac{\partial F_{0}}{\partial y}\right)=\left(0, a_{21} x+a_{31} x^{2}+2 a_{32} x y\right. & \\
& \left.+a_{41} x^{3}+2 a_{42} x^{2} y+3 a_{43} x y^{2}+4 a_{44} y^{3}\right) .
\end{aligned}
$$

We shall work downwards on jet levels and start by showing that all monomials $\left(x^{i} y^{j}, 0\right)$ and $\left(0, x^{i} y^{j}\right)$ with $i+j \leq 4$ are in the left hand side of

$$
j^{k}\left(L \mathcal{A}_{e} \cdot F_{0}+\mathbb{R} \cdot\{\dot{F}\}\right)=J^{k}(2,2) .
$$

For any monomial $Q(x, y)$ of degree $\leq 4$ the terms $(Q(x, y), 0)$ is in the left hand side of (5.16), because $(Q(x, y), 0)=Q(x, y) \frac{\partial F_{0}}{\partial x}$. We observe that, using the first component of $F$, the monomials $\left(x^{i}, 0\right)$ and $\left(0, x^{i}\right)$ are in $L \mathcal{L}_{e} \cdot F_{0}$ and hence in the left hand side of (5.16). We get all 
monomials of degree 4 of the form $\left(0, Q_{1}(x, y)\right)$ using $j^{4} Q_{1}(x, y) \frac{\partial F_{0}}{\partial x}$ where $Q_{1}$ is of degree 3 . We also get $\left(0, x^{2} y\right)$ from $j^{4} x^{2} \frac{\partial F_{0}}{\partial x}$. We have

$$
\left(0, a_{44} y^{4}\right) \equiv j^{3}\left(x \frac{\partial F_{0}}{\partial x}\right)-j^{3}(0, f(x, y))
$$

As $a_{44} \neq 0$, we get $\left(0, y^{4}\right)$. Now, $j^{3}\left(y^{2} \frac{\partial F_{0}}{\partial y}\right) \equiv\left(0, a_{21} x y^{2}\right)$, similarly, $j^{3}\left(x \frac{\partial F_{0}}{\partial x}\right) \equiv\left(0, a_{21} x y\right)$, which give $\left(0, x y^{2}\right)$ and $(0, x y)$ as $a_{21} \neq 0$. We get $\left(0, y^{3}\right)$ from $j^{3} \frac{\partial F_{0}}{\partial y}$ and $\left(0, y^{2}\right)$ from $\dot{F}$. Finally, we get $(0, y)$ from $j^{3} \frac{\partial F_{0}}{\partial x}$. Thus, $F$ is $\mathcal{A}_{e}$-versal unfolding of the swallowtail singularity.

The critical set for the family $\tilde{P}(x, y, u, 0)$ is the set

$$
\Sigma\left(\tilde{P}_{w}\right)=\left\{(x, y) \in \mathbb{R}^{2} \mid a_{21} x+O(2)=0\right\}
$$

As $a_{21} \neq 0$, by the implicit function theorem we can write locally $x$ as a function of $y$ and $u$ with

$$
x(y, u)=u y-\frac{4 a_{44}}{a_{21}} y^{3}+\frac{2 a_{20}}{a_{21}} u^{2} y-\frac{a_{32}}{a_{21}} u y^{2}+O(4) .
$$

Substituting (5.18) in $\tilde{P}(x, y, u, 0)$ we get an expression for the profile which has 4-jet equal to

$$
\left(2 u y-\frac{a_{32}}{a_{21}} u y^{2}-\frac{4 a_{44}}{a_{21}} y^{3},-3 a_{44} y^{4}+a_{21} u y^{2}\right) .
$$

Because $\tilde{P}(x, y, u, 0)$ is $\mathcal{A}_{e}$-versal the profile (5.19) undergoes the swallowtail transition.

Theorem 5.4. For a given surface $M$ in $\mathbb{R}^{3}$ suppose that $P_{w_{0}}$ has swallowtail singularity at $p$ where $w_{0}=(0,1,0)$. Then

(i) The point $p$ is a felecnodal point and $w$ is an asymptotic direction at $p$.

(ii) The proper evolute of the profile of a swallowtail singularity is an ordinary cusp with the cusp point coinciding with the swallowtail point. The full evolute of the profile is the proper evolute together with 4 lines coinciding with the limiting normal line of the profile at the swallowtail singularity (see Figure 5.2 center).

(iii) The bifurcation in the full evolute of the profile at a swallowtail singularity is as in Figure 5.2.

Proof. For item (i) we refer reader to [20]. We take $M$ locally in Monge form with $f$ as in (5.1). We also consider the family $\tilde{P}(x, y, u, 0)$.

The curvature of the profile is given by

$$
\kappa_{c}=\frac{4 a_{21}^{2} u^{2}-12 a_{21} a_{32} u^{2} y+\left(12 a_{32}^{2} u^{2}-48 a_{21} a_{44} u\right) y^{2}+96 a_{32} a_{44} u y^{3}+144 a_{44}^{2} y^{4}+O(5)}{\left(\left(2 u+\frac{-4 a_{32}}{a_{21}} y+\frac{-12 a_{44}}{a_{21}} y^{2}+O(3)\right)^{2}+\left(2 a_{21} u y-3 a_{32} u y^{2}-12 a_{44} y^{3}+O(4)\right)^{2}\right)^{\frac{3}{2}}} .
$$


Using (5.19), we get the expression for the proper evolute of the profile,

$$
e(y)=\left(\frac{-12 a_{44} a_{21}+32 a_{44}+4 a_{21} u}{a_{21}} y^{3}+O\left(y^{4}\right), u-\frac{\left(6 a_{21} u+36 a_{44}\right)}{2} y^{2}+O\left(y^{3}\right)\right),
$$

which has one ordinary cusp singularity at $\tilde{P}_{w}(0,0)$ for all $u$ near zero. Therefore, away from the singularity points of the profile, there is one single vertex.

Using the numerator of curvature of the profile we conclude that the maximum number of inflections in the bifurcation of profile is 4 . Also the numerator of first derivative of curvature is given by

$$
\begin{array}{r}
O\left(u^{4}\right)+O\left(u^{3}\right) y+O\left(u^{3}\right) y^{2}+O\left(u^{2}\right) y^{3}+O\left(u^{2}\right) y^{4}+\left(20736 a_{21} a_{44}^{3} u+O\left(u^{2}\right)\right) y^{5} \\
-41472 a_{44}^{4} y^{7}+O(8) .
\end{array}
$$

Hence, we have a maximum of 7 vertices. According to $\$ 3.2$, we know that there is an accumulation of 2 inflections and 3 vertices at each cusp singularity. Thus, we do not expect to observe inflection in the bifurcation (which is true because according to item (i) swallowtail happens at the flecnodal curve hence generically the bifurcation of critical set of $\tilde{P}_{w}$ does not intersect the parabolic curve).

Using expression (5.19), we can locate the cusps of the profile at $y=y_{1}$ and $y=y_{2}$. Dividing the numerator of the first derivative of the curvature by $\left(y-y_{1}\right)^{3}\left(y-y_{2}\right)^{3}$, we obtain another point $y=y_{3}$ which indicates the vertex point in the bifurcation of the swallowtail singularity. A straightforward calculation shows that $\kappa_{c}\left(y_{3}\right) \kappa^{\prime \prime}\left(y_{3}\right)=3072 a_{21}^{7} a_{44} u^{7}+O\left(u^{8}\right)$ this implies that we have a change from an outward vertex to an inward vertex in the bifurcation of the swallowtail singularity (for precise details of calculations see the Appendix C).

For the rest of proof, using similar calculations to those in the proof of Theorem 3.4 part (ii), we get the full evolute (when $u=0$ ) as the proper evolute together with 4 lines located in the normal direction at the point $\tilde{P}_{w}(0,0)$.

\subsection{Lips/Beaks}

When the point $p \in M$ is a parabolic point and $w$ is an asymptotic direction then $\tilde{P}$ has a lips/beaks singularity. This singularity has $\mathcal{A}_{e}$-codimension one.

Proposition 5.3. Let $F(x, y, v)=\tilde{P}(x, y, 0, v)$ be a 1-parameter family of a lips/beaks singularity. Then the family $F$ is an $\mathcal{A}_{e}$-versal family.

Proof. The proof is similar to proof of Proposition 5.2 and Theorem 6.8 of [24] and is omitted. 


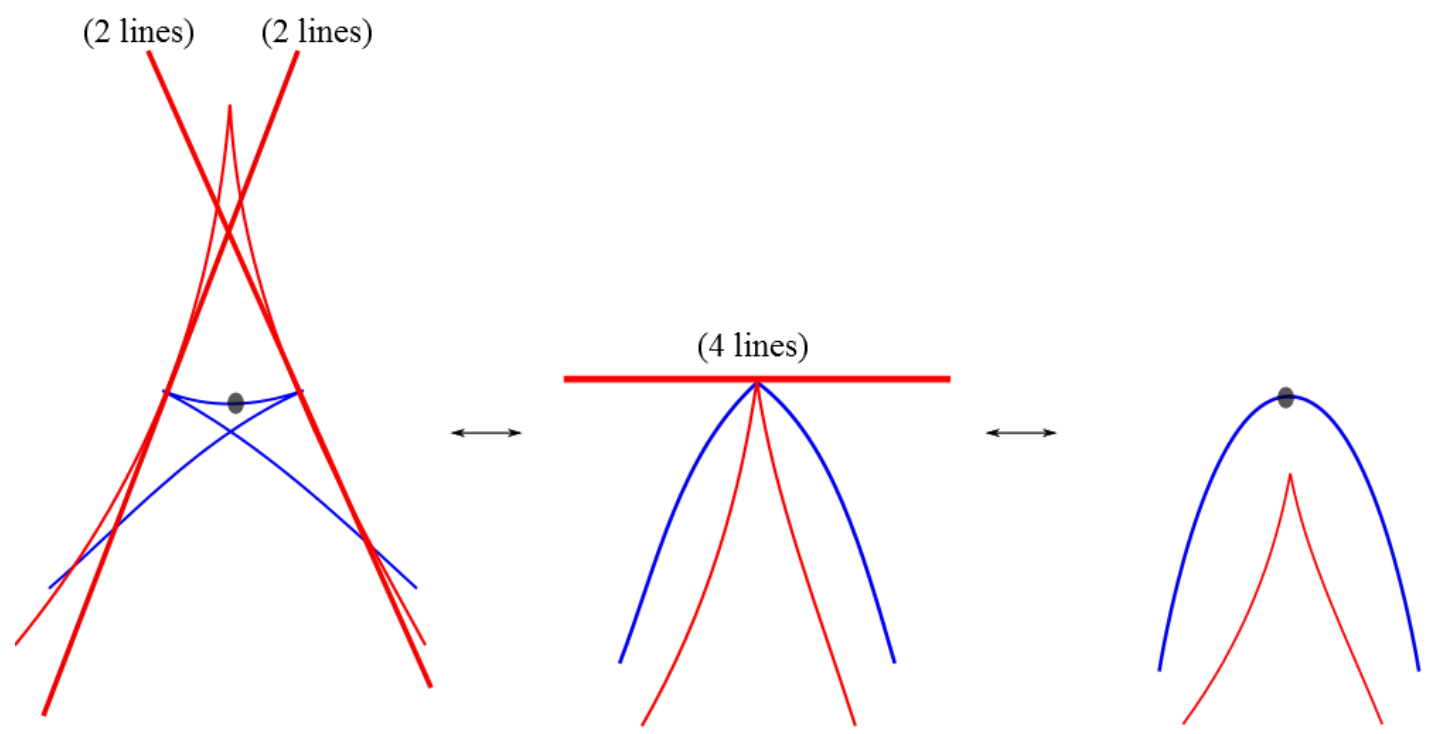

Figure 5.2 - The bifurcation in the evolute of the profile at a swallowtail singularity. Dots represents the vertex points.

Koenderink's theorem shows that if $\kappa_{n}(w) \neq 0$ then $K=\kappa_{n}(w) \kappa_{c}$, where $\kappa_{n}(w)$ is the normal curvature along $w$ at $p \in M$ and $\kappa_{c}$ is the curvature of the profile of $M$ along $w$ at a point corresponding to $p$.

Set

$$
\tilde{K}(x, y)=\bar{l}(x, y) \bar{n}(x, y)-\bar{m}(x, y)^{2}
$$

where

$$
\bar{l}=\left\langle\phi_{x x}, \phi_{x} \times \phi_{y}\right\rangle, \quad \bar{m}=\left\langle\phi_{x y}, \phi_{x} \times \phi_{y}\right\rangle, \quad \bar{n}=\left\langle\phi_{y y}, \phi_{x} \times \phi_{y}\right\rangle .
$$

One can observe that $K=0$ if and only if $\tilde{K}=0$. Hence, the intersection points of $\tilde{K}(x, y)=0$ and $\Sigma\left(\tilde{P}_{w}\right)$ correspond to inflections of the profile along $w$.

If $\kappa_{n}(w)=0$, that is, $w$ is asymptotic at $p$ then the profile along $w$ has a cusp at a point corresponding to $p$. Set

$$
C(x, y)=a^{2} \bar{l}(x, y)+2 a b \bar{m}(x, y)+b^{2} \bar{n}(x, y)
$$

for a tangent vector $w=a \phi_{x}(x, y)+b \phi_{y}(x, y)$. The zero set $C(x, y)=0$ is the locus of points where $w$ is asymptotic. Therefore, intersections between $C(x, y)=0$ and $\Sigma\left(\tilde{P}_{w}\right)$ correspond to cusps of the profile along $w$.

To analyze the vertices of the profile, we consider cylindrical directions. These are defined in [17] as follows.

Definition 5.2. Assume that a surface $M$ is given in Monge form $\phi(x, y)=(x, y, f(x, y))$ at the origin with $f$ as in (5.1). We denote the cubic part of $f$ by $f_{3}(x, y)$. The tangent direction at the origin along $w=a \phi_{x}(0,0)+b \phi_{y}(0,0)$ is a cylindrical direction at the origin if $f_{3}(\bar{a}, \bar{b})=0$, where $\bar{w}=\bar{a} \phi_{x}(0,0)+\bar{b} \phi_{y}(0,0)$ is the conjugate of $w$ at the orign. 
If $M$ is given in Monge form $z=f(x, y)$ with $f$ as in (5.1) then we have

$$
\begin{aligned}
\kappa_{1}(0,0) & =2 a_{20}, \quad \kappa_{2}(0,0)=2 a_{22}, \\
w_{1} \kappa_{1}(0,0)=6 a_{30}, \quad w_{2} \kappa_{1}(0,0) & =2 a_{31}, \quad w_{1} \kappa_{2}(0,0)=2 a_{32}, \quad w_{2} \kappa_{2}(0,0)=6 a_{33},
\end{aligned}
$$

where $w_{i} \kappa_{j}$ is the directional derivative of $\kappa_{j}$ along $w_{i}$. Hence, $f_{3}$ can be written in the form

$$
f_{3}=w_{1} \kappa_{1}(0,0) x^{3}+w_{2} \kappa_{1}(0,0) x^{2} y+w_{1} \kappa_{2}(0,0) x y^{2}+w_{2} \kappa_{2}(0,0) y^{3} .
$$

By these assumptions we can extend the definition of cylindrical direction at a point close to origin.

Definition 5.3. Let $M$ be parameterized by a smooth map $\phi$, and let $w=a \phi_{x}(p)+b \phi_{y}(p)$ and $\bar{w}=\bar{a} \phi_{x}(p)+\bar{b} \phi_{y}(p)$ be conjugate of $w$ at $\phi(p)$. A tangent direction at $\phi(p)$ generated by $w$ is a cylindrical direction at $\phi(p)$ if

$$
w_{1} \kappa_{1}(p) \bar{a}^{3}+w_{2} \kappa_{1}(p) \bar{a}^{2} \bar{b}+w_{1} \kappa_{2}(p) \bar{a} \bar{b}^{2}+w_{2} \kappa_{2}(p) \bar{b}^{3}=0 .
$$

Remark 5.3. Assume that $\phi(p)$ is not a parabolic point and $w$ is not an asymptotic direction at $\phi(p)$. Then $w$ is cylindrical at $\phi(p)$ if and only if $M$ has $A_{\geq 3}$-contact with a cylinder whose axis is parallel to $w$ and whose radius is $\lambda=\kappa_{n}(w) / K(p)$ at $\phi(p)$, where $\kappa_{n}(w)$ is the normal curvature along $w$ at $\phi(p)$ (see Proposition 4.11 in [17]). Moreover, this condition is equivalent to the condition that the profile of $M$ along $w$ has $A_{\geq 3}$-contact with a circle whose radius is $\lambda$ at a point q corresponding point to $\phi(p)$ (Proposition 4.12 in [17]).

A plane curve has a vertex at a point if and only the curve has $A_{\geq 3}$-contact with a circle there. Therefore, $w$ is a cylindrical direction at $\phi(p)$ if and only if the profile along $w$ has a vertex at a point corresponding to $\phi(p)$. Let tangent vectors $w=a \phi_{x}(x, y)+b \phi_{y}(x, y)$ and $\bar{w}=\bar{a} \phi_{x}(x, y)+\bar{b} \phi_{y}(x, y)$ are conjugate. For $\bar{w}$ set

$$
V(x, y)=w_{1} \kappa_{1}(x, y) \bar{a}^{3}+w_{2} \kappa_{1}(x, y) \bar{a}^{2} \bar{b}+w_{1} \kappa_{2}(x, y) \bar{a} \bar{b}^{2}+w_{2} \kappa_{2}(x, y) \bar{b}^{3} .
$$

The zero set $V(x, y)=0$ is the locus of points where $w$ is cylindrical. So, the points of intersection between $V(x, y)=0$ and $\Sigma\left(\tilde{P}_{w}\right)$ correspond to vertices of the profile along $w$.

Theorem 5.5. For a given surface $M$ in $\mathbb{R}^{3}$ let the modified orthogonal projection $\tilde{P}_{w_{0}}$ has lips/beaks singularity where $w_{0}=(0,1,0)$. Then

i) There is a birth of two inflections and two vertices in the bifurcation of the profile at a lip singularity.

ii) There are 3 different beaks type singularities. In the first type, which we call it $D_{4}^{+}$-beak, there is a birth of 2 inflections and 2 vertices on one side and no inflections and no vertices on the other side of bifurcation of the profile. In the second type, which we call it $D_{4}^{-}$-beak, 
there is a birth of 2 inflections and 4 vertices on one side and 2 vertices and no inflection on the other side of bifurcation of the profile. The third beak type is called $D_{5}$-beak and happens at an isolated point on the parabolic set of the surface $M$.

Proof. We take the surface $M$ locally in Monge form parametrisation $z=f(x, y)$ with $f$ as in (5.1). Let us investigate the configuration of $\Sigma\left(\tilde{P}_{w}\right), \tilde{K}(x, y)=0, C(x, y)=0$, and $V(x, y)=0$ for lips and beaks. Set $w_{0}=(0,1,0)$ and $w=(0,1, v)$.

Suppose that the type of singularity of $\tilde{P}_{w_{0}}$ is lips or beaks. Then we have

$$
a_{20} \neq 0, \quad a_{21}=a_{22}=0, \quad a_{33} \neq 0, \quad 3 a_{31} a_{33}-a_{32}^{2} \neq 0 .
$$

Remark that the singularity of $\tilde{P}_{w_{0}}$ is lips (resp. beaks) if and only if $3 a_{31} a_{33}-a_{32}^{2}>0$ (resp. $<0)$. The critical set $\Sigma\left(\tilde{P}_{w}\right)$ is given by

$$
\Sigma\left(P_{w}\right)=\left\{f_{y}(x, y)=v\right\}=\left\{-v+a_{31} x^{2}+2 a_{32} x y+3 a_{33} y^{2}+O(3)=0\right\} .
$$

It undergoes Morse transition in Figure 5.3 left (resp. Figure 5.3 right) if the type of singularity of $\tilde{P}_{w_{0}}$ is lips (resp. beaks).
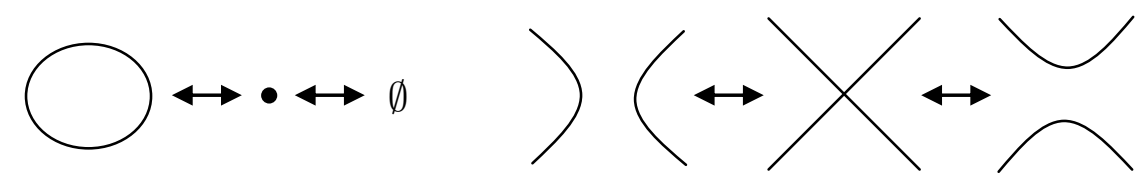

Figure 5.3 - The two types of Morse transitions.

Straightforwad calculations show that

$$
\begin{aligned}
\tilde{K}(x, y)= & 4 a_{20} a_{32} x+12 a_{20} a_{33} y+4\left(a_{31}^{2}-3 a_{30} a_{32}-a_{20} a_{42}\right) x^{2} \\
& +4\left(a_{31} a_{32}-9 a_{30} a_{33}-3 a_{20} a_{43}\right) x y+4\left(a_{32}^{2}-3 a_{31} a_{33}-6 a_{20} a_{44}\right) y^{2}+O(3) .
\end{aligned}
$$

Since $w=(0,1, v)$ can be written as $w=a \phi_{x}+b \phi_{y}$, we have

$$
a=0, \quad b=1, \quad f_{y}(x, y)=v .
$$

We remark that the locus $f_{y}(x, y)=v$ gives $\Sigma\left(\tilde{P}_{w}\right)$ in the parameter space. Then

$$
C(x, y)=\bar{n}(x, y)=2 a_{32} x+6 a_{33} y+2 a_{42} x^{2}+6 a_{43} x y+12 a_{44} y^{2}+O(3) .
$$

It follows from (5.23) and (5.24) that the vector $\left(1,-\frac{a_{32}}{3 a_{33}}\right)$ is tangent to both curves $\tilde{K}(x, y)=0$ and $C(x, y)=0$ at the origin. Moreover, since $\tilde{K}(x, y)=0$ divides the parameter space near the origin into the hyperbolic and elliptic region, $\tilde{K}(x, y)=0$ and $C(x, y)=0$ have $2 k$-point $(k \geq 1)$ contact at the origin. Actually, when $3 a_{31} a_{33}-a_{32}^{2} \neq 0$ these two curves have 2-point 
contact at the origin because (5.24) shows that $C(x, y)=0$ can be parameterized near the origin by

$$
c(t)=\left(t,-\frac{a_{32}}{3 a_{33}} t+\frac{-3 a_{33}^{2} a_{42}+3 a_{32} a_{33} a_{43}-2 a_{32}^{2} a_{44}}{9 a_{33}^{2}} t^{2}+O(3)\right) .
$$

Therefore we have

$$
\left.(\tilde{K} \circ c(t))^{\prime}\right|_{t=0}=0,\left.\quad(\tilde{K} \circ c(t))^{\prime \prime}\right|_{t=0}=8\left(a_{32}^{2}-3 a_{31} a_{33}\right) \neq 0 .
$$

We have

$$
\begin{aligned}
V(x, y)=- & 48\left(\left(a_{30} a_{32}^{3}-a_{31}^{3} a_{33}\right) x^{3}\right. \\
+ & a_{32}\left(a_{31} a_{32}^{2}-6 a_{31}^{2} a_{33}+9 a_{30} a_{33}\right) x^{2} y \\
+ & \left(a_{32}^{4}-3 a_{31} a_{32}^{2} a_{33}-9 a_{31}^{2} a_{33}^{2}+27 a_{30} a_{32} a_{33}^{2}\right) x^{2} y \\
& \left.+a_{33}\left(2 a_{32}^{3}-9 a_{31} a_{32} a_{33}+27 a_{30} a_{33}^{2}\right) y^{3}\right)+O(4) .
\end{aligned}
$$

We denote the cubic part of $V$ by $V_{3}$. The discriminant of $V_{3}$ is given by

$$
\Delta=2^{16} \cdot 3^{4}\left(3 a_{31} a_{33}-a_{32}^{2}\right)^{6} \Delta^{\prime}
$$

where

$$
\Delta^{\prime}=3 a_{31}^{2} a_{32}^{2}-4 a_{30} a_{32}^{3}-4 a_{31}^{3} a_{33}+18 a_{30} a_{31} a_{32} a_{33}-27 a_{30}^{2} a_{33}^{2} .
$$

We remark that $\Delta^{\prime}$ coincides with the discriminant of the cubic part of $f$ as in (5.1). Since

$$
\Delta^{\prime}=-27 a_{32}^{2}\left(a_{30}+\frac{2 a_{32}^{3}-9 a_{31} a_{32} a_{33}}{27 a_{33}^{2}}\right)^{2}-\frac{4\left(3 a_{31} a_{33}-a_{32}^{2}\right)^{3}}{27 a_{33}^{2}},
$$

if $3 a_{31} a_{33}-a_{32}^{2}>0$ then $\Delta^{\prime}<0$ which is equivalent to $\Delta<0$. On the other hand, if $3 a_{31} a_{33}-a_{32}^{2}<0$ then $\Delta$ can take negative, positive and zero values.

Now we consider the case of lips, so we have $3 a_{31} a_{33}-a_{32}^{2}>0$. Since $\Delta<0, V$ has a $D_{4}^{+}$-singularity at the origin, thus $V(x, y)=0$ is locally a smooth curve passing through the origin. Moreover, the zero set $V(x, y)=0$ is transverse to both zero sets $\tilde{K}(x, y)=0$ and $C(x, y)=0$ at the origin because we have

$$
V_{3}\left(1,-\frac{a_{32}}{3 a_{33}}\right)=\frac{16\left(3 a_{31} a_{33}-a_{32}^{2}\right)^{3}}{9 a_{33}^{2}} \neq 0 .
$$

It follows that the arrangement of $\Sigma\left(\tilde{P}_{w_{0}}\right), \tilde{K}(x, y)=0, C(x, y)=0$ and $V(x, y)=0$ is as in Figure 5.4 and the arrangement of cusps, inflections, and vertices on $\Sigma\left(\tilde{P}_{w_{0}}\right)$ is shown in Figure 5.5. Remark that $\Sigma\left(\tilde{P}_{w_{0}}\right)$ is an isolated point. Hence, the bifurcation of the profile along $w$ is as in Figure 5.6.

Now we consider the case of beaks, so $3 a_{31} a_{33}-a_{32}^{2}<0$. The critical set $\Sigma\left(\tilde{P}_{w_{0}}\right)$ has two local branches which are smooth curves and intersect transversally at the origin. We have $\Sigma\left(\tilde{P}_{w_{0}}\right)$ given by

$$
\Sigma\left(\tilde{P}_{w_{0}}\right)=\left\{a_{31} x^{2}+2 a_{32} x y+3 a_{33} y^{2}+O(3)=0\right\} .
$$




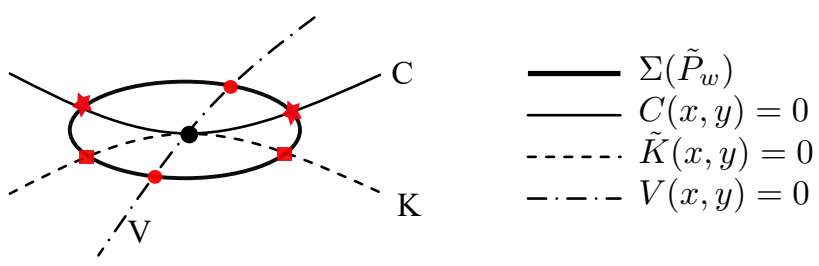

Figure 5.4 - The arrangement of $\Sigma\left(\tilde{P}_{w_{0}}\right), \tilde{K}(x, y)=0, C(x, y)=0$, and $V(x, y)=0$.

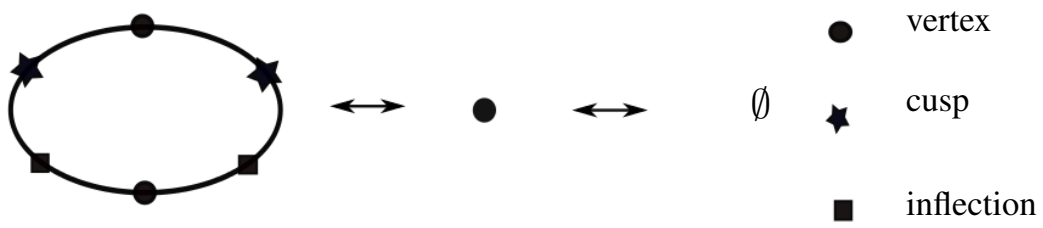

Figure 5.5 - The arrangemant of cusps, inflections, and vertices on $\Sigma\left(\tilde{P}_{w_{0}}\right)$.

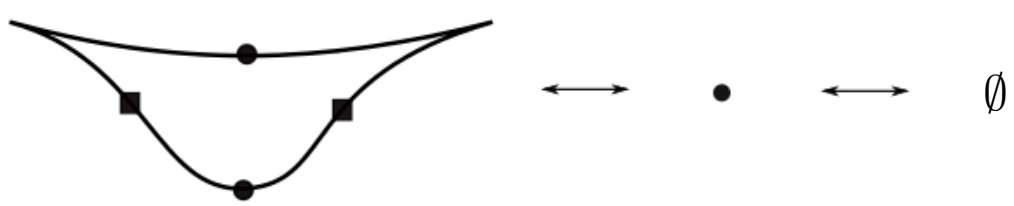

Figure 5.6 - The bifurcation of the profile at a lip singularity. Discs represent vertex points and full squares represent inflection points.

Since $a_{33} \neq 0$, the branches can be expressed as the zero sets of

$$
\begin{aligned}
& P(x, y)=\frac{-a_{32}+\sqrt{a_{32}^{2}-3 a_{31} a_{33}} x-y+O(2) \quad \text { and }}{3 a_{33}} x-\sqrt{a_{32}^{2}-3 a_{31} a_{33}} x-y+O(2) . \\
& 3 a_{33}
\end{aligned}
$$

The vectors

$$
\left(1, \alpha_{1}\right)=\left(1, \frac{-a_{32}+\sqrt{a_{32}^{2}-3 a_{31} a_{33}}}{3 a_{33}}\right)
$$

and

$$
\left(1, \alpha_{2}\right)=\left(1,-\frac{a_{32}+\sqrt{a_{32}^{2}-3 a_{31} a_{33}}}{3 a_{33}}\right),
$$

are tangent respectively to $P(x, y)=0$ and $Q(x, y)=0$ at the origin. Let $C_{1}$ denotes the linear term of $C$, namely, $C_{1}(x, y)=2 a_{32} x+6 a_{33} y$. The zero set $C_{1}(x, y)=0$ locally divides the parameter space into two regions $\left\{C_{1}(x, y)>0\right\}$ and $\left\{C_{1}(x, y)<0\right\}$. Since

$$
C_{1}\left(1, \alpha_{1}\right) C_{1}\left(1, \alpha_{2}\right)=4\left(3 a_{31} a_{33}-a_{32}^{2}\right)<0
$$

the vectors $\left(1, \alpha_{1}\right)$ and $\left(1, \alpha_{2}\right)$ lie in different regions. Hence, the arrangement of $\Sigma\left(\tilde{P}_{w_{0}}\right)$, $C(x, y)=0$, and $\tilde{K}(x, y)=0$ is as in Figure 5.7. 


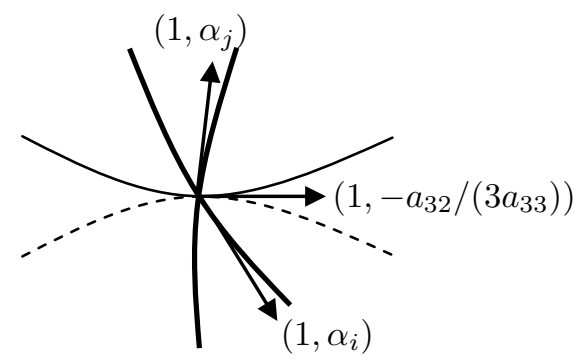

Figure 5.7 - The arrangement of $\Sigma\left(\tilde{P}_{w_{0}}\right), \tilde{K}(x, y)=0, C(x, y)=0$, and $V(x, y)=0$.

Firstly, we assume that $\Delta<0$, i.e., $\Delta^{\prime}<0$ i.e, the curve $V(x, y)$ has $D_{4}^{+}$-singularity at the origin (this is why we called this type the $D_{4}^{+}$-beaks). As mentioned in the case of lips, $V(x, y)=0$ is locally a smooth curve and transverse to the both curve $\tilde{K}(x, y)=0$ and $C(x, y)=0$ at the origin.

The zero set $V_{3}(x, y)=0$ divides the region near the origin into two region $\left\{V_{3}(x, y)>0\right\}$ and $\left\{V_{3}(x, y)<0\right\}$. We have

$$
V_{3}\left(1, \alpha_{1}\right) V_{3}\left(1, \alpha_{2}\right)=-\frac{256\left(3 a_{31} a_{33}-a_{32}^{2}\right)^{3} \Delta^{\prime}}{3 a_{33}^{2}}<0 .
$$

This meas that $\left(1, \alpha_{1}\right)$ and $\left(1, \alpha_{2}\right)$ lie in different regions with respect to $V_{3}=0$. So the arrangement of $\Sigma\left(\tilde{P}_{w_{0}}\right), \tilde{K}(x, y)=0, C(x, y)=0$, and $V(x, y)=0$ is as in Figure 5.8 and the arrangement of cusps, inflections, and vertices on $\Sigma\left(\tilde{P}_{w}\right)$ is as in Figure 5.9. Hence, the bifurcation of the profile along $w$ is as in Figure 5.10.

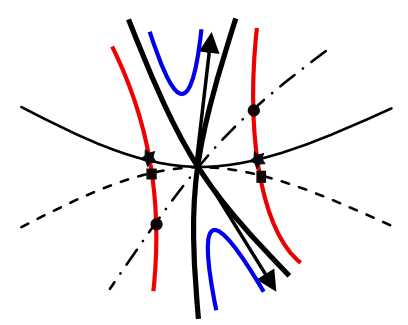

Figure 5.8 - The arrangement of $\Sigma\left(\tilde{P}_{w_{0}}\right), \tilde{K}(x, y)=0, C(x, y)=0$, and $V(x, y)=0$ at the $D_{4}^{+}$-beaks.

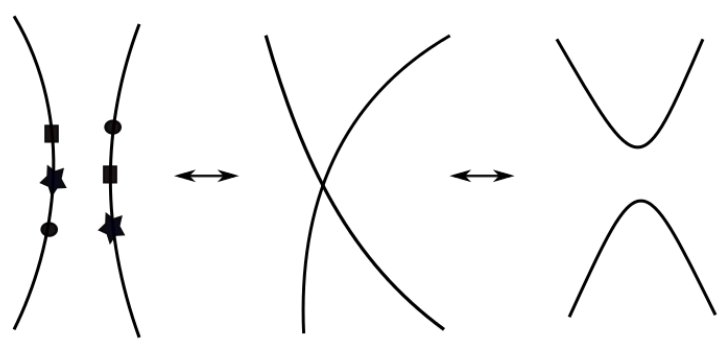

Figure 5.9 - The arrangemant of cusps, inflections, and vertices on $\Sigma\left(\tilde{P}_{w}\right)$ at the $D_{4}^{+}$-beaks.

Now we assume that $\Delta>0$, i.e., $\Delta^{\prime}>0$. Then $V$ has a $D_{4}^{-}$-singularity at the origin (we call this beaks type $D_{4}^{-}$-beaks) and thus $V(x, y)=0$ has locally three smooth transverse branches 


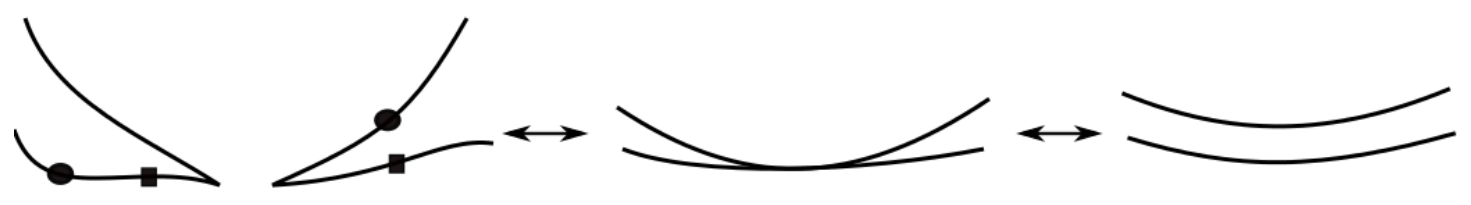

Figure 5.10 - The bifurcation of the profile along $w$ when $\tilde{P}_{w_{0}}$ has $D_{4}^{+}$-beaks singularity.

passing through the origin. The zero set $V_{3}(x, y)=0$ divides the region near the origin into six regions, three $\left\{V_{3}(x, y)>0\right\}$ and three $\left\{V_{3}(x, y)<0\right\}$. Since $3 a_{31} a_{33}-a_{32}^{2}<0,(5.29)$ shows that $V_{3}\left(1,-\frac{a_{31}}{3 a_{33}}\right)<0$. Hence, $\left(1,-\frac{a_{32}}{3 a_{33}}\right)$ belongs to a region $\left\{V_{3}(x, y)<0\right\}$. Since $\Delta^{\prime}>0$, it follows from (5.31) that we have $V_{3}\left(1, \alpha_{1}\right) V_{3}\left(1, \alpha_{2}\right)>0$. Moreover, we have

$$
\begin{aligned}
& V_{3}\left(1, \alpha_{1}\right)=\frac{16\left(a_{32}^{2}-3 a_{31} a_{33}\right)\left(\left(2 a_{32}^{3}-9 a_{31} a_{32} a_{33}+27 a_{30} a_{33}^{2}\right) \sqrt{a_{32}^{2}-3 a_{31} a_{33}}+2\left(a_{32}^{2}-3 a_{31} a_{33}\right)^{2}\right)}{9 a_{33}^{2}}, \\
& V_{3}\left(1, \alpha_{2}\right)=\frac{16\left(a_{32}^{2}-3 a_{31} a_{33}\right)\left(-\left(2 a_{32}^{3}-9 a_{31} a_{32} a_{33}+27 a_{30} a_{33}^{2}\right) \sqrt{a_{32}^{2}-3 a_{31} a_{33}}+2\left(a_{32}^{2}-3 a_{31} a_{33}\right)^{2}\right)}{9 a_{33}^{2}} .
\end{aligned}
$$

If $2 a_{32}^{3}-9 a_{31} a_{32} a_{33}+27 a_{30} a_{33}^{2} \geq 0$, then $V_{3}\left(1, \alpha_{1}\right)>0$, and thus $V_{3}\left(1, \alpha_{2}\right)>0$ because $V_{3}\left(1, \alpha_{1}\right) V_{3}\left(1, \alpha_{2}\right)>0$. On the other, if $2 a_{32}^{3}-9 a_{31} a_{32} a_{33}+27 a_{30} a_{33}^{2}<0$, then We conclude similarly that $V_{3}\left(1, \alpha_{1}\right)>0$ and $V_{3}\left(1, \alpha_{2}\right)>0$. Therefore, we always have $V_{3}\left(1, \alpha_{1}\right)>0$ and $V_{3}\left(1, \alpha_{2}\right)>0$. Accordingly, the arrangement of $V_{3}(x, y)=0,\left(1,-\frac{a_{32}}{3 a_{33}}\right)$, and $\left(1, \alpha_{i}\right)$ is as in Figure 5.11. Hence, the arrangement of $\Sigma\left(\tilde{P}_{w_{0}}\right), C(x, y)=0, \tilde{K}(x, y)=0$ and $V(x, y)=0$ is as in Figure 5.12. From Figure 5.12, it follows that the arrangement of cusps, inflections, and vertices on $\Sigma\left(\tilde{P}_{w}\right)$ is as in 5.13, and thus the bifurcation of the profile along $w$ is as in Figure 5.14 .

When $\Delta=0$ i.e. $V(x, y)$ has $D_{5}$-singularity. Therefore, this happens at an isolated point on the parabolic set. We call this $D_{5}$-beaks singularity.

Remark 5.4. According to Theorem 4.2 of [17] the sub-parabolic curve passes through an isolated point on parabolic set where $D_{5}$-beaks singularity happens at this point.

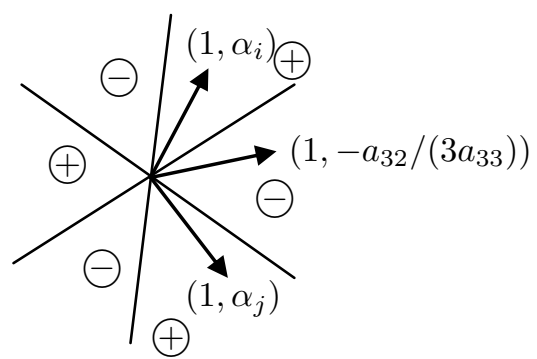

Figure 5.11 - The arrangement of $V_{3}(x, y)=0,\left(1,-\frac{a_{32}}{3 a_{33}}\right)$, and $\left(1, \alpha_{i}\right)$.

\subsubsection{Evolute of the profile at a lips/beaks singularity}

In this part we study the evolute of the profile when $\tilde{P}_{w}$ has a lips/beaks singularity.

Theorem 5.6. For a given surface $M$ in $\mathbb{R}^{3}$ let $\tilde{P}_{w_{0}}$ has a lips/beaks singularity. Then 


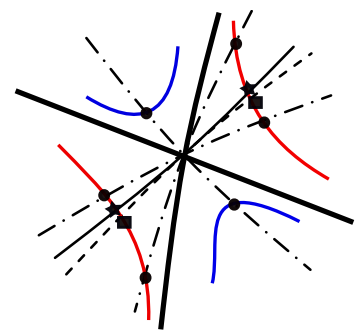

Figure 5.12 - The arrangement of $\Sigma\left(\tilde{P}_{w_{0}}\right), C(x, y)=0, \tilde{K}(x, y)=0$, and $V(x, y)=0$ at the $D_{4}^{-}$-beaks.

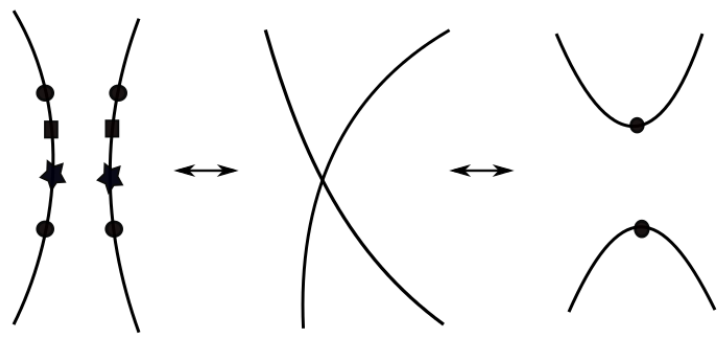

Figure 5.13 - The arrangement of cusps, inflections, and vertices on $\Sigma\left(\tilde{P}_{w}\right)$ at the $D_{4}^{-}$-beaks.

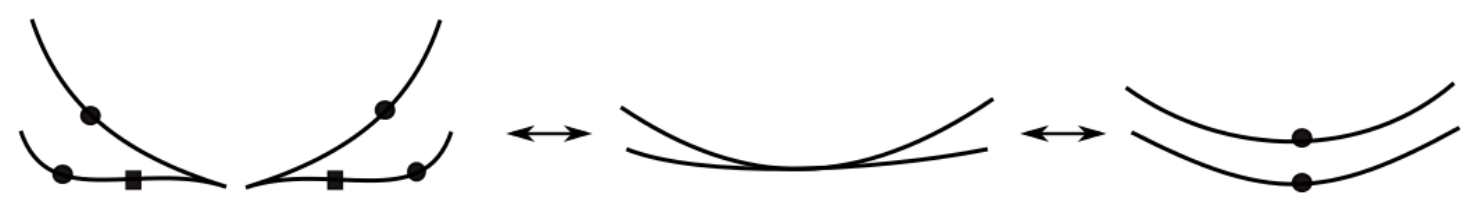

Figure 5.14 - The bifurcation of the profile along $w$ when $\tilde{P}_{w_{0}}$ has $D_{4}^{-}$-beaks singularity.

i) There is no line in the full evolute of the profile of a lip singularity (Figure 5.15 center). The bifurcation of the evolute of the profile at a lip singularity is given in Figure 5.15.

ii) The proper evolute of the profile of a $D_{4}^{+}$-beak (resp. $D_{4}^{-}$-beak) consists of two tangential parabola passing through the point $\left(0, \frac{1}{2 a_{20}}\right)$ see Figure 5.16 (resp. Figure 5.17) center. The full evolute of the profile of a $D_{4}^{+}$-beak (resp. $D_{4}^{-}$-beak) singularity is the proper evolute together with 6 lines coinciding at the limiting normal line of beaks point and tangent to the proper evolute at the point $\left(0, \frac{1}{2 a_{20}}\right)$.

Proof. Using information on the evolute at an inflection point and at a cusp point, one can obtain the bifurcation in the full evolute at a lips/beaks singularity as is given in Figure 5.15. Note that as we are considering the real number case so the full evolute at an isolated point is an empty set.

At a $D_{4}^{+}$-beak singularity, we have 2 cusps, 2 inflections and 2 vertices on one side of the transition and regular curves with no points of inflections or vertices on the other side of the transition (see Figure 5.10). We can find the expression of each branch of the critical set in beaks singularity as in (5.30). Substituting (5.30) in the projection map one can obtain the expression 


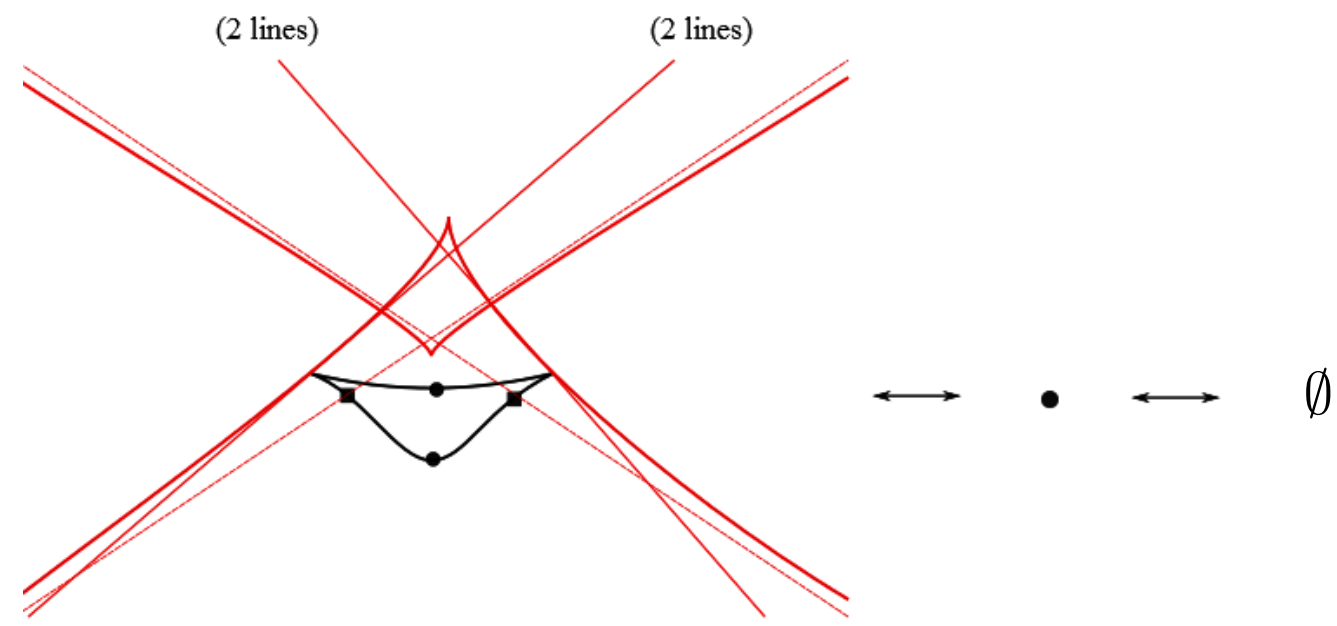

Figure 5.15 - The bifurcation of the evolute of profile along $w$ when $\tilde{P}_{w_{0}}$ has lips singularity.

of profile as follow:

$$
\begin{aligned}
& P\left(x, \alpha_{1} x+O\left(x^{2}\right)\right)=(x, \underbrace{a_{20} x^{2}+\left(a_{30}+a_{31} \alpha_{1}+a_{32} \alpha_{1}^{2}+a_{33} \alpha_{1}^{3}\right) x^{3}+O\left(x^{4}\right)}_{P_{1}(x)}), \\
& P\left(x, \alpha_{2} x+O\left(x^{2}\right)\right)=(x, \underbrace{a_{20} x^{2}+\left(a_{30}+a_{31} \alpha_{2}+a_{32} \alpha_{2}^{2}+a_{33} \alpha_{2}^{3}\right) x^{3}+O\left(x^{4}\right)}_{P_{2}(x)}) .
\end{aligned}
$$

Consider the function $g(x, y)=\left(y-P_{1}(x)\right)\left(y-P_{2}(x)\right)$. The profile can be locally defined by $g(x, y)=0$. To find the full evolute of the profile of $\tilde{P}_{w_{0}}$ we consider the bifurcation set of the family of distance squared functions

$$
D=(x-a)^{2}+(y-b)^{2}-\eta g(x, y) .
$$

We have

$$
\begin{aligned}
D_{x}=2(x-a)-\eta g_{x} & \Rightarrow a=\frac{1}{2}\left(2 x-\eta g_{x}\right), \\
D_{y}=2(y-b)-\eta g_{y} & \Rightarrow b=\frac{1}{2}\left(2 y-\eta g_{y}\right), \\
D_{\eta}=-g & \Rightarrow y=P_{1}(x) \quad \text { or } \quad y=P_{2}(x) .
\end{aligned}
$$

The Hessian matrix of $D$ is given by

$$
\mathcal{H}(D)=\left(\begin{array}{ccc}
2-\eta g_{x x} & -\eta g_{x y} & -g_{x} \\
-\eta g_{x y} & 2-\eta g_{y y} & -g_{y} \\
-g_{x} & -g_{y} & 0
\end{array}\right) .
$$

The determinant of (5.33) is equal to

$$
\operatorname{det}(\mathcal{H}(D))=\underbrace{-2\left(g_{x}^{2}+g_{y}^{2}\right)}_{B}+\eta \underbrace{\left(g_{y y} g_{x}^{2}-2 g_{x y} g_{x} g_{y}+g_{x x} g_{y}^{2}\right)}_{A} .
$$


Substituting $y=P_{1}(x)$ into $A$ and $B$ in (5.34) we get

$$
\begin{aligned}
& A=-\frac{128\left(a_{20}\left(a_{32}^{2}-3 a_{31} a_{33}\right)^{9 / 2}\right)}{19683 a_{33}^{6}} x^{9}+O\left(x^{10}\right), \\
& B=-\frac{32\left(a_{32}^{2}-3 a_{31} a_{33}\right)^{3}}{729 a_{33}^{4}} x^{6}+O\left(x^{7}\right) .
\end{aligned}
$$

Equating (5.34) to zero we get $\eta=-\frac{B_{6} x^{6}+B_{7} x^{7}+O\left(x^{8}\right)}{A_{9} x^{9}+A_{10} x^{10}+O\left(x^{11}\right)}$, where $B_{i}$ (resp. $A_{i}$ ) denotes the coefficient of $x^{i}$ in $B$ (resp. $A$ ). After simplifying $\eta$ we get $\eta=-\frac{B_{6}+B_{7} x+O\left(x^{2}\right)}{x^{3}\left(A_{9}+A_{10} x+O\left(x^{2}\right)\right)}$. If we substitute $y=P_{2}(x)$ into $A$ and $B$ we get also $\eta=-\frac{\bar{B}_{6}+\bar{B}_{7} x+O\left(x^{2}\right)}{x^{3}\left(\bar{A}_{9}+\bar{A}_{10} x+O\left(x^{2}\right)\right)}$. This means that 6 lines appear as a part of the full evolute. By straightforward calculation one can get the proper evolute given by

$$
\begin{aligned}
& a=\frac{1}{18 a_{20} a_{33}^{2}}\left(2 a_{32}^{3}-9 a_{31} a_{32} a_{33}+27 a_{30} a_{33}^{2}+2\left(a_{32}^{2}-3 a_{31} a_{33}\right)^{\frac{3}{2}}\right) x^{2}+O\left(x^{3}\right), \\
& b=\frac{1}{2 a_{20}}-\frac{1}{18 a_{20}^{2} a_{33}^{2}}\left(2 a_{32}^{2}-9 a_{31} a_{32} a_{33}+27 a_{30} a_{33}^{2}+2\left(a_{32}^{2}-3 a_{31} a_{33}\right)^{\frac{3}{2}}\right) x+O\left(x^{2}\right) .
\end{aligned}
$$

Therefore the proper evolute is a pair of parabolas (for each branch we got one parabola) passing through $\left(0, \frac{1}{2 a_{20}}\right)$ and tangent to limiting normal line of beaks point as shown in Figure 5.16 center.

Similar calculations hold for $D_{4}^{-}$-beak (see Figure 5.17).

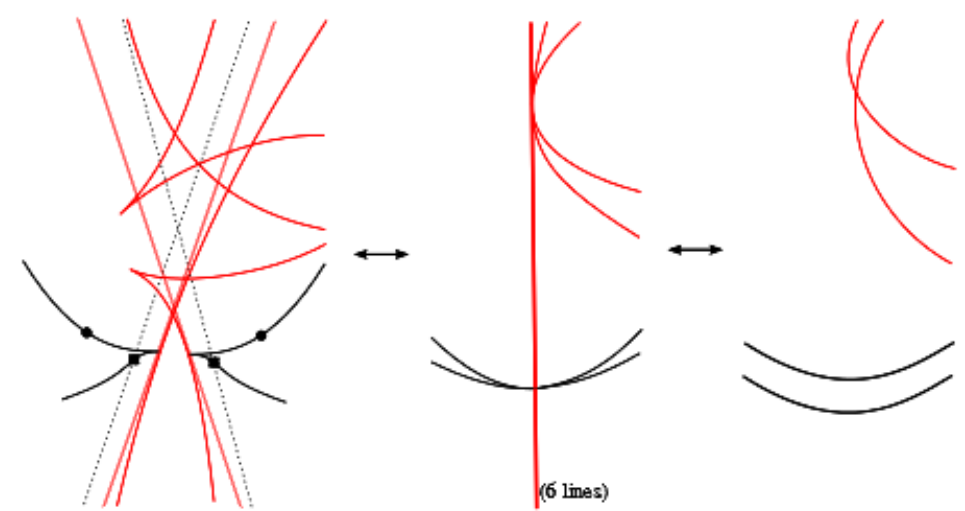

Figure 5.16 - The bifurcation of the full evolute of the profile at a $D_{4}^{+}$-beaks singularity. Discs represent vertex points and full squares represent inflections.

\subsection{Butterfly}

Suppose that the given surface $M$ is parametrized by the Monge form $(x, y, f(x, y))$ with $f(x, y)$ as in (5.1). According to Theorem 6.7 in [24], the algebraic conditions to have butterfly 


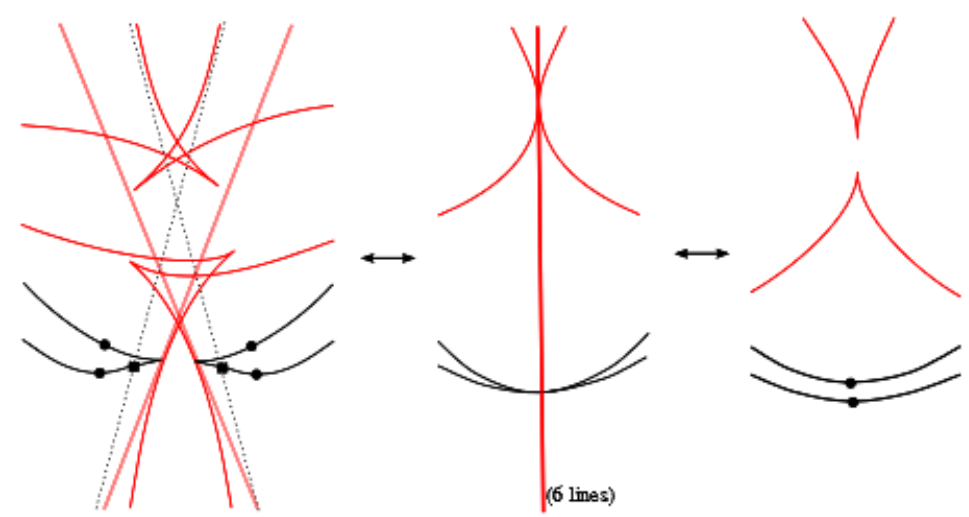

Figure 5.17 - The bifurcation of the full evolute of the profile at a $D_{4}^{-}$-beaks singularity. Discs represent vertices and full squares represent inflection points.

are

$$
\begin{aligned}
& a_{22}=0, a_{33}=0, a_{44}=0, a_{21} \neq 0, a_{55} \neq 0, \\
& \left(8 a_{55} a_{77}-5 a_{66}^{2}\right) a_{21}^{2}+2 a_{55}\left(a_{32} a_{66}-20 a_{43} a_{55}\right) a_{21}+35 a_{32}^{2} a_{55}^{2} \neq 0 .
\end{aligned}
$$

Let $\tilde{P}_{w}$ be the modified family of orthogonal projections of $M$ as in 5.8 .

Proposition 5.4 (Theorem 6.8 in [24]). The modified family of the orthogonal projections (5.8), is 2-parameter $\mathcal{A}_{e}$-versal unfolding at a butterfly singularity if the flecnodal curve is not singular.

Theorem 5.7. For a given regular surface $M$ let $\tilde{P}_{w}$ denotes the modified orthogonal projection of $M$ as in 5.8. If $\tilde{P}_{w_{0}}$ with $w_{0}=(0,1,0)$ has a butterfly singularity then

(i) The point $p$ is a flecnodal point, $w_{0}$ an asymptotic direction at $p$ and tangent to flecnodal curve at $p$.

(ii) The bifurcation diagram of the profile at a butterfly singularity is given in Figure 5.18. There are at most 2 ordinary vertices and no inflections in the bifurcation of the profile, one is outward and the other is inward vertex. These are as in Figure 5.18.

(iii) The bifurcation in the evolute at a butterfly singularity is given in Figure 5.19.

Proof. For (i) see [20]. For (ii) the critical set $\Sigma\left(\tilde{P}_{w}\right)$ at a butterfly singularity is given by

$$
\left\{(x, y) \in \mathbb{R}^{2} \mid a_{21} x+O(2)=0\right\}
$$


Thus, since $a_{21} \neq 0$ by the implicit function theorem, we obtain $x=x(y, u, v)$. Substituting $x$ in $\tilde{P}_{w}$ we get the expression for the profile given by $(X(y, u, v), Y(y, u, v))$ with

$$
\begin{aligned}
X(y, u, v)= & -\frac{v}{a_{21}}+\left(\frac{2 u+2 a_{32} v}{a_{21}^{2}}\right) y+\left(-\frac{a_{32} u}{a_{21}}+\frac{\left(3 a_{21} a_{43}-4 a_{32}^{2}\right) v}{a_{21}^{3}}\right) y^{2} \\
& +\left(-\frac{2\left(a_{21} a_{43}-a_{32}^{2}\right) u}{a_{21}^{2}}+\frac{4\left(a_{21}^{2} a_{54}-3 a_{21} a_{32} a_{43}+2 a_{32}^{3}\right) v}{a_{21}^{4}}\right) y^{3} \\
& -\frac{5 a_{55}}{a_{21}} y^{4}+O(5), \\
Y(y, u, v)= & \left(a_{21} u+\frac{a_{32} v}{a 21}\right) y^{2}+\left(\frac{\left(3 a_{21} a_{43}-4 a_{32}^{2}\right)}{a_{21}^{2}}+\frac{2 a_{32}^{2}}{a_{21}^{2}}-\frac{a_{43}}{a_{21}}\right) v y^{3}+O(u, v) y^{4} \\
& +\left(-4 a_{55}+O(u, v)\right) y^{5}+\left(-\frac{5 a_{32} a_{55}}{a_{21}}+a_{66}+O(u, v)\right) y^{6} \\
& +\left(\frac{-5 a_{43} a_{55}}{a_{21}}+a_{77}+O(u, v)\right) y^{7}+O(8) .
\end{aligned}
$$

The bifurcation diagram of the profile at the butterfly singularity has the following strata:

1. The swallowtail stratum $(S W)$ happens when

$$
\frac{\partial g}{\partial y}=\frac{\partial^{2} g}{\partial y^{2}}=\frac{\partial^{3} g}{\partial y^{3}}=0
$$

where $g$ is the second component of $\tilde{P}(x-u y, y, u, v)$. The calculation can be carried out with the help of a computer algebra package such as Maple or Mathematica (see Appendix D for Maple codes). We found that the swallowtail stratum is parametrized by

$$
\left(\frac{-30}{a_{55}} y^{3}+O\left(y^{4}\right), \frac{1}{a_{21}} y^{2}+O\left(y^{3}\right)\right) .
$$

Hence the $(S W)$-stratum is locally a cusp (see (4) and (14) of Figure 5.18).

2. The multi-local stratum $((F C))$ : This occurs when the projection $\tilde{P}_{w}$ has a cusp at $\left(x_{1}, y_{1}\right)$ and a fold at $\left(x_{2}, y_{2}\right)$ with $g_{w}\left(x_{1}, y_{1}\right)=g_{w}\left(x_{2}, y_{2}\right)$ and $\left(x_{1}, y_{1}\right) \neq\left(x_{2}, y_{2}\right)\left(g_{w}(x, y)=g(x, y, u, v)\right.$ as defined in the (SW)-stratum). Here we obtain $x_{1}=x_{2}$ and $y_{1} \neq y_{2}$. For a fold at $\left(x_{1}, y_{2}\right)$ we need $\frac{\partial g_{w}}{\partial y}\left(x_{1}, y_{2}\right)=0$ and for a cusp at $\left(x_{1}, y_{1}\right)$ we need $\frac{\partial g_{w}}{\partial y}\left(x_{1}, y_{1}\right)=\frac{\partial^{2} g_{w}}{\partial y^{2}}\left(x_{1}, y_{1}\right)=0$. Therefore, considering $g_{w}$ as a function of $y$ for a given $x_{1}=x_{2}=x$, there exists a real $\omega$ such that $g_{w}(x, y)-\omega$ has a repeated root and a triple root. Therefore we have

$$
g_{w}(x, y)-\omega=\left(y-y_{1}\right)^{2}\left(y-y_{3}\right)^{3}\left(\theta y^{2}+\alpha y+\beta\right) .
$$

Comparing coefficients of this with $g_{w}(x, y)$ gives $u=\bar{a} y_{2}^{3}+O(4)$ and $v=\bar{b} y_{2}^{2}+O(3)$ with $\bar{a}=\frac{-200 a_{21} a_{55}^{2}}{a_{21} a_{43}-a_{32}^{2}}$ and $\bar{b}=\frac{10 a_{55} a_{21}^{2}}{a_{21} a_{43}-a_{32}^{2}}$ (note that according to Observation 5.4 the flecnodal curve is not singular, equivalently $\tilde{P}_{w}$ is $\mathcal{A}_{e}$-versal. According to Theorem 6.8 of [24], an algebraic condition to have $\mathcal{A}_{e}$-versality of a butterfly singularity is $a_{32}^{2}-a_{21} a_{43} \neq 0$ ).

3. The $(V T)$-stratum: Here a vertex in one branch of the profile intersects transversally another branch of the profile. This stratum is captured by the singularities of distance squared function 
on the profile which is given by

$$
D=(X(y, u, v)-a)^{2}+(Y(y, u, v)-b)^{2} .
$$

The solution of system of equations

$$
\left\{\begin{array}{l}
X\left(y_{1}, u, v\right)=X\left(y_{2}, u, v\right) \\
Y\left(y_{1}, u, v\right)=Y\left(y_{2}, u, v\right) \\
\frac{\partial D}{\partial y_{1}}\left(y_{1}, u, v\right)=0 \\
\frac{\partial^{2} D}{\partial y_{1}^{2}}\left(y_{1}, u, v\right)=0 \\
\frac{\partial^{3} D}{\partial y_{1}^{3}}\left(y_{1}, u, v\right)=0,
\end{array}\right.
$$

gives us the desire stratum. By a direct calculations, using implicit function theorem, we get the following parametrisation of the stratum

$$
(u, v)=\left(-\frac{10 a_{55}}{3} y_{1}^{3}+O(4), 2 a_{55} y_{1}^{2}+O(3)\right) .
$$

4. The second order vertex stratum $(S V)$ : Here the profile along $w$ has a second order vertex, so $\kappa_{c}^{\prime}(y)=\kappa_{c}^{\prime \prime}(y)=0$. Using the implicit function theorem we obtain a local parametrization giving by $\left(\frac{70 a_{55}}{11} t^{3}+O(4),-\frac{10 a_{55}}{11} t^{2}+O(3)\right)$ for this stratum (see (16) and (2) of Figure 5.18).

Using the numerator of the curvature and its first derivative of the profile, we get a maximum of 6 inflections and 11 vertices in the bifurcation of the profile at a butterfly singularity. As there is an accumulation of 3 vertices and 2 inflections in cusp points and since there are 3 cusps in the bifurcation of profile of a butterfly singularity, so all the inflections and 9 vertices are concentrated at the cusp points. Therefore there are just 2 vertices in the bifurcation of the profile at a butterfly singularity. As seen in $§ 3.1$, when we pass through the second order vertex stratum two vertices appear which one of these vertices is outward and the other is inward vertex.

(iii) Following the behavior on each stratum and using the evolute at an ordinary inflection (Figure 3.3), ordinary cusp (Figure 3.9) and second order vertex (Figure 3.2) we draw the bifurcation in the evolute of the profile at a butterfly singularity as in Figure 5.19. Note that at the origin using similar calculations to those in the proof of Theorem 3.4 part (ii), we get the line $a=0$ with multiplicity 6 as part of the bifurcation set of the family of distance squared functions $D$. The proper evolute is given by $(a, b)=\left(\frac{15 a_{55}}{a_{21}} y^{4}+O\left(y^{5}\right),-\frac{20 a_{55}}{a_{21}^{2}} y^{3}+O\left(y^{4}\right)\right)$.

\subsection{Goose}

According to Theorem 6.7 of [24], the algebraic conditions at a goose singularity are

$$
\begin{aligned}
& a_{22}=0, a_{21}=0, a_{32}^{2}-3 a_{31} a_{33}=0, a_{33} \neq 0, \\
& \delta=27 a_{41} a_{33}^{3}-18 a_{42} a_{32} a_{33}^{2}+9 a_{43} a_{32}^{2} a_{33}-4 a_{44} a_{32}^{3} \neq 0 .
\end{aligned}
$$




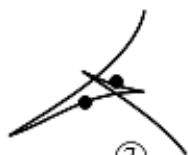

(7)

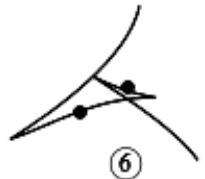

(6)

(1)

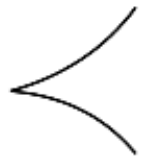

(4)

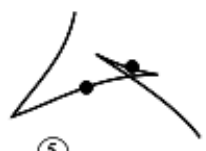

(5)

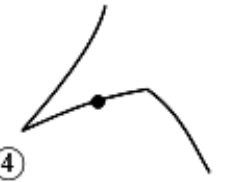

(3)

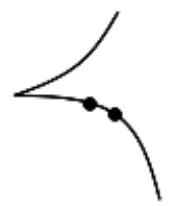

(2)

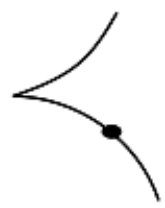

(15)

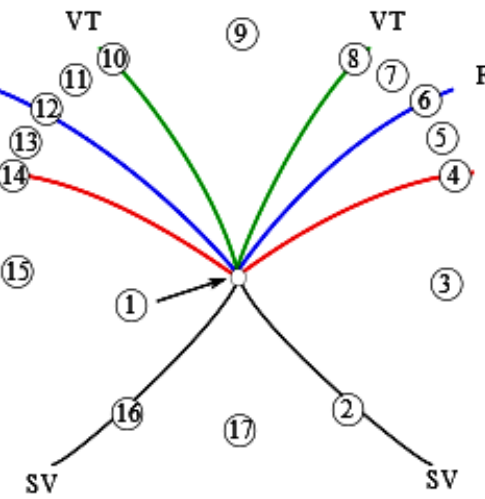

SW

(3)

(16)

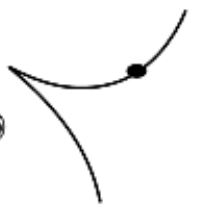

(17)

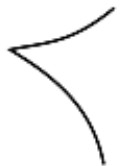

(12)

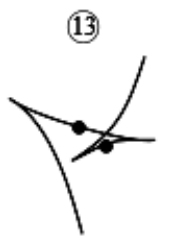

(14)

(15)

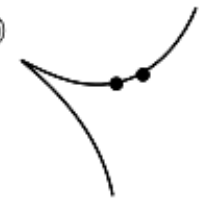

Figure 5.18 - The bifurcation of the profile at a butterfly singularity. Full discs represent vertex points.

Let $\tilde{P}_{w}$ be the modified orthogonal projection of surface $M$ along $w$ as mentioned in (5.8). The following observation shows the condition about $\mathcal{A}_{e}$-versality of $\tilde{P}_{w}$ at a goose .

Observation 5.1 (Theorem 6.8 of [24]). If the orthogonal projection of surface $M$ along unit vector $w_{0}$ has a goose singularity then the family of modified orthogonal projections $\tilde{P}_{w}$ is an $\mathcal{A}_{e}$-versal if and only if $a_{20} \neq 0$.

Since the point $p \in M$ is not flat umblic, so $a_{20} \neq 0$. Hence according to Observation 5.1 the family of modified orthogonal projections at a goose singularity is $\mathcal{A}_{e}$-versal. 

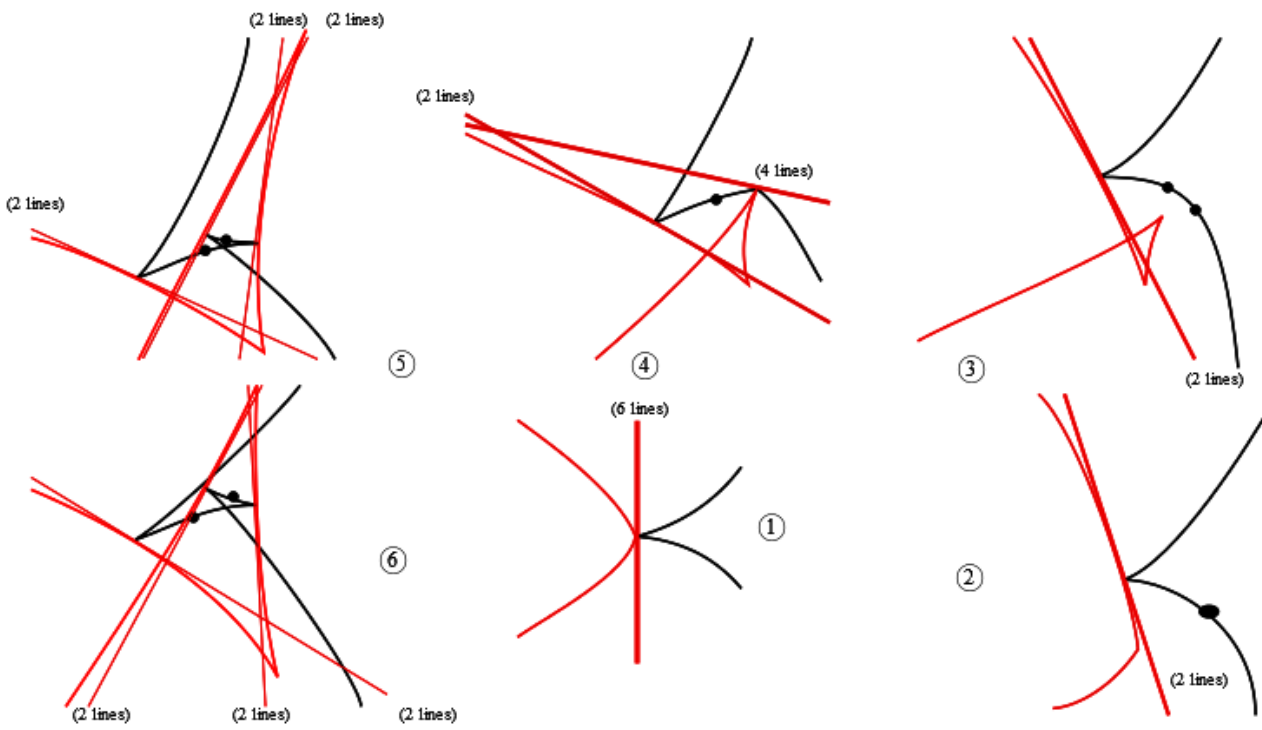

(1)

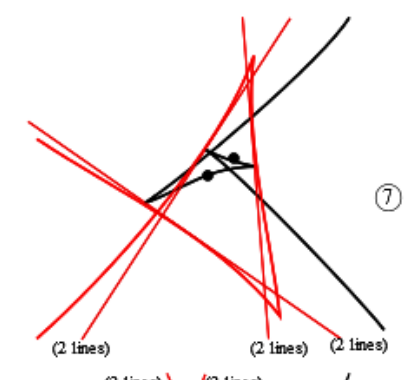

(7)

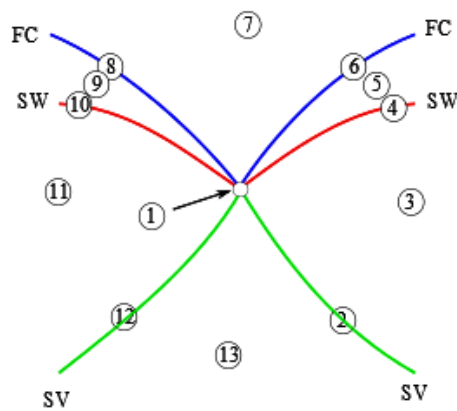

(13)
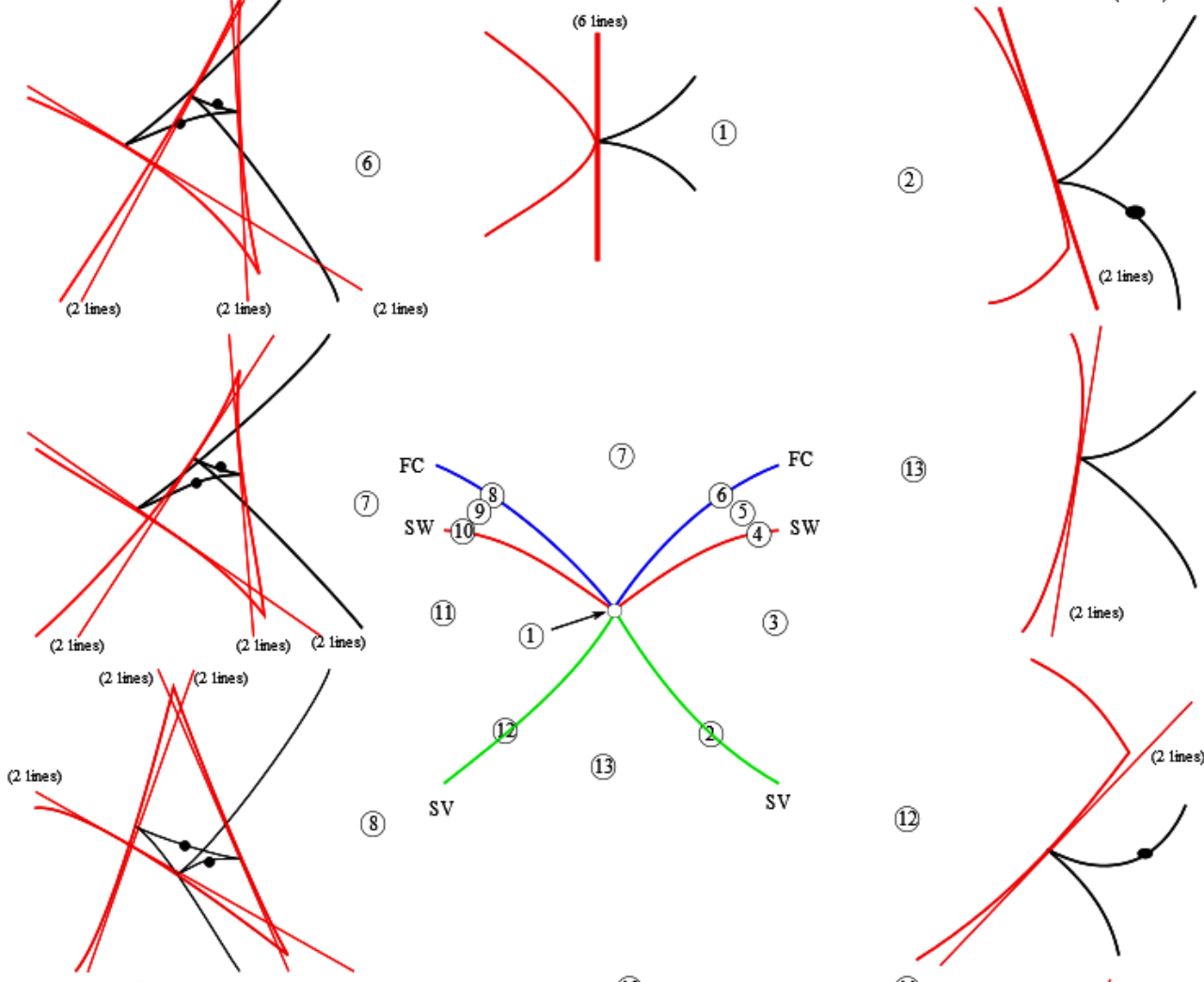

SV

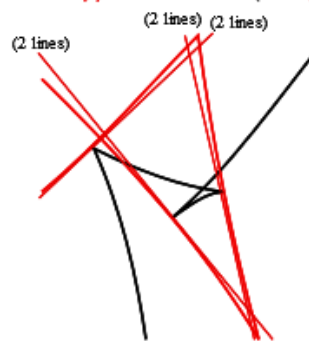

(9)

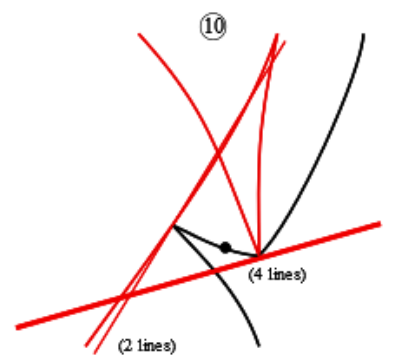

(2)

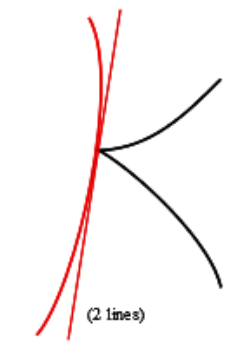

Figure 5.19 - The bifurcation in the evolute of the profile at a butterfly singularity.

The critical set $\Sigma\left(\tilde{P}_{w}\right)$ at a goose singularity is given by

$$
\begin{aligned}
& v-2 a_{20} x u+\left(-3 a_{30} u+\frac{a_{32}^{2}}{3 a_{33}}\right) x^{2}+\left(2\left(\frac{-2 a_{32}^{2} u}{3 a_{33}}+a_{32}\right)\right) y x+\left(3\left(-a_{32} u+a_{33}\right)\right) y^{2} \\
& +\left(-4 a_{40} u+a_{41}\right) x^{3}+\left(2\left(-3 a_{41} u+a_{42}\right)\right) y x^{2}+\left(3\left(-2 a_{42} u+a_{43}\right)\right) y^{2} x \\
& +\left(4\left(-a_{43} u+a_{44}\right)\right) y^{3}+O(4)=0
\end{aligned}
$$

After performing the change of coordinate $y \mapsto y-\frac{a_{32}}{3 a_{33}} x$ on (5.38), the critical set (5.38) becomes $3 a_{33} y^{2}+\delta x^{3}+O(4)=0$ where $\delta$ is given in (5.37). Subsequently, a local parametrization for 
the critical set given by $y=c_{1} t^{3}+O\left(t^{4}\right)$ and $x=d_{1} t^{2}+O\left(t^{3}\right)$ such that $c_{1} \neq 0$ and $d_{1}=$ $\left(\frac{-81 a_{33}^{4} c_{1}^{2}}{\delta}\right)^{(1 / 3)}$. Substituting this parametrization into $\tilde{P}_{w}$ we get the profile at $(u, v)=(0,0)$, $\mathcal{A}$-equivalent to

$$
\left(t^{2}, \beta_{9} t^{9}\right)
$$

where

$$
\beta_{9}=\frac{-1458.3^{2 / 3} a_{33}^{9} c_{1}^{7}}{\left(\frac{-a_{33}^{4} c_{1}^{2}}{\delta}\right)^{1 / 3} \delta^{2}} \neq 0 .
$$

Theorem 5.8. For a given surface $M$ in $\mathbb{R}^{3}$ let $\tilde{P}_{w}$ be the family of modified orthogonal projections of $M$ as in 5.8. If $\tilde{P}_{w_{0}}$ has a goose singularity at $p \in M$ where $w_{0}=(0,1,0)$, then

(i) The point $p$ is a parabolic point, $w_{0}$ an assymptotic direction at $p$ and the Gauss image of the parabolic set has a geodesic inflection.

(ii) There are at most 3 ordinary vertices and 3 ordinary inflections in the bifurcation of the profile at a goose singularity.

(iii) The bifurcation of the full evolute of the profile at a goose singularity is given in Figure 5.21 .

Proof. For the surface $M$ we take the Monge form parametrisation $\phi(x, y)=(x, y, f(x, y))$ with $f$ as in (5.1). For (i) see Theorem 6.7 of [24]. For (ii) and (iii) let $F(x, y, u, v)=\tilde{P}(x-u y, y, u, v)$ and $g$ denotes the second component of $F$. The following stratum appear in the bifurcation of the profile at a goose singularity.

Lips/beaks-stratum (L/B): This happens when critical set $\Sigma\left(\tilde{P}_{w}\right)$ is singular. This means that we have $\frac{\partial g}{\partial y}=\frac{\partial^{2} g}{\partial y^{2}}=\frac{\partial^{2} g}{\partial y \partial x}=0$. A direct calculation using the implicit function theorem, leads to the following parametrization for the $(L / B)$-stratum:

$$
(u, v)=\left(\frac{\delta}{18 a_{20} a_{33}^{3}} t^{2}+O\left(t^{3}\right), \frac{2 \delta}{27 a_{33}^{3}} t^{3}+O\left(t^{4}\right)\right) .
$$

One way to study the geometry of the profile at a goose singularity is to consider intersection of critical set $\Sigma\left(\tilde{P}_{w}\right)$ with the curves $C(x, y)=0, \tilde{K}(x, y)=0$ and $V(x, y)=0$.

We have $w=a \phi_{x}+b \phi_{y}$ therefore after performing the changes of coordinate $u \mapsto u+\frac{\pi}{2}$ and $v \mapsto v+\frac{\pi}{2}$ we get $a=-\cos (v) \sin (u)$ and $b=\cos (v) \cos (u)$. By a similar calculation to those in Theorem 5.5, one can obtain the expression of the curves $\tilde{K}(x, y)=0, C(x, y)=0$ and $V(x, y)=0$ as follow:

$$
\begin{aligned}
C(x, y)= & -\frac{2}{9}\left(\frac{9 a_{20} a_{33}^{2} \cos (u)^{2}-9 a_{20} a_{33}^{2}}{a_{33}^{2}}\right)-\frac{2}{9}\left(\frac{27 \cos (u)^{2} a_{30} a_{33}^{2}-\cos (u)^{2} a_{32}^{3}-27 a_{30} a_{33}^{2}+a_{32}^{3}}{a_{33}^{2}}\right) x \\
& -\frac{2}{9}\left(\frac{18 \sin (u) \cos (u) a_{32} a_{33}^{2}+3 \cos (u)^{2} a_{32}^{2} a_{33}-27 \cos (u)^{2} a_{33}^{3}-3 a_{32}^{2} a_{33}}{a_{33}^{2}}\right) y+O(2), \\
\tilde{K}(x, y)= & 12 a_{20} a_{33} y+O(2), \\
V(x, y)= & -96 a_{32} \cos (v)^{3} \sin (u)^{2} a_{20}^{3}\left(a_{32} \sin (u)-\sin (u) a_{33}-3 a_{33} \cos (u)\right) y+O(2) .
\end{aligned}
$$


The critical set at the origin has cusp singularity. Hence, locally the critical set is a cubic polynomial and the curves $C(x, y), \tilde{K}(x, y)$ and $V(x, y)$ are smooth curves (away from the origin). Therefore locally the maximum number of cusps, ordinary inflections and ordinary vertices in the bifurcation of the profile at a goose singularity is 3 .

Note that as seen in $\S 5.5$, we have only one type of lips singularity which the discriminant of vertex curve $V(x, y)=0$ has $D_{4}^{+}$-singularity. Therefore in (L/B)-stratum the $D_{4}^{+}$-beak happens.

As mentioned in (5.39) at the origin the profile is a (2-9)-cusp. The full evolute of such a singularity captured by considering the bifurcation of family of distance squared functions

$$
D_{(a, b)}(t)=\left(t^{2}-a\right)^{2}+\left(\beta_{4} t^{4}+\beta_{6} t^{6}+\beta_{8} t^{8}+\beta_{9} t^{9}-b\right)^{2},
$$

where

$$
\beta_{4}=3^{\frac{8}{3}} a_{20}\left(\frac{a_{33}^{4} c_{1}^{2}}{(-\delta)^{\frac{2}{3}}}\right) \neq 0
$$

A direct calculation shows that the proper evolute of the profile at the origin has the following parametrization.

$$
e(t)=\left(\frac{3 \beta_{6}}{2 \beta_{4}} t^{4}-\frac{64 \beta_{4}^{4}-64 \beta_{8} \beta_{4}+72 \beta_{6}^{2}}{\beta_{4}^{2}} t^{6}+\frac{45 \beta_{9}}{8 \beta_{4}} t^{7}+O(8), \frac{1}{\beta_{4}}-\frac{3 \beta_{6}}{2 \beta_{4}^{2}} t^{2}+O(3)\right) .
$$

The full evolute is given by the proper evolute together with 3 lines coinciding with the limiting normal line of the proper evolute, passing through the point $\left(0, \frac{1}{\beta_{4}}\right)$.

The rest of the proof is a matter of considering the behavior of the evolute at an inflection point, the evolute at a lip singularity and the evolute at a $D_{4}^{+}$-beak singularity as given in Figures 5.15, 5.16 respectively (see Figure 5.21).

\subsection{Gulls}

Suppose that the surface $M$ is parametrised by the Monge form parametrization $\phi(x, y)=$ $(x, y, f(x, y))$, where $f$ is given by (5.1). A modified orthogonal projection of $M$ has gulls singularity if

$$
\begin{aligned}
& a_{22}=0, a_{21}=0, a_{33}=0, a_{32} \neq 0, a_{44} \neq 0, \\
& a_{55} a_{32}^{2}-2 a_{43} a_{44} a_{32}+4 a_{31} a_{44}^{2} \neq 0 .
\end{aligned}
$$

The gulls singularity happens when the point $p=\phi(0,0)$ is the cusp of Gauss and the direction $w$ is asymptotic. The following observation gives a necessary and sufficient condition for versality of a family of modified projections. 

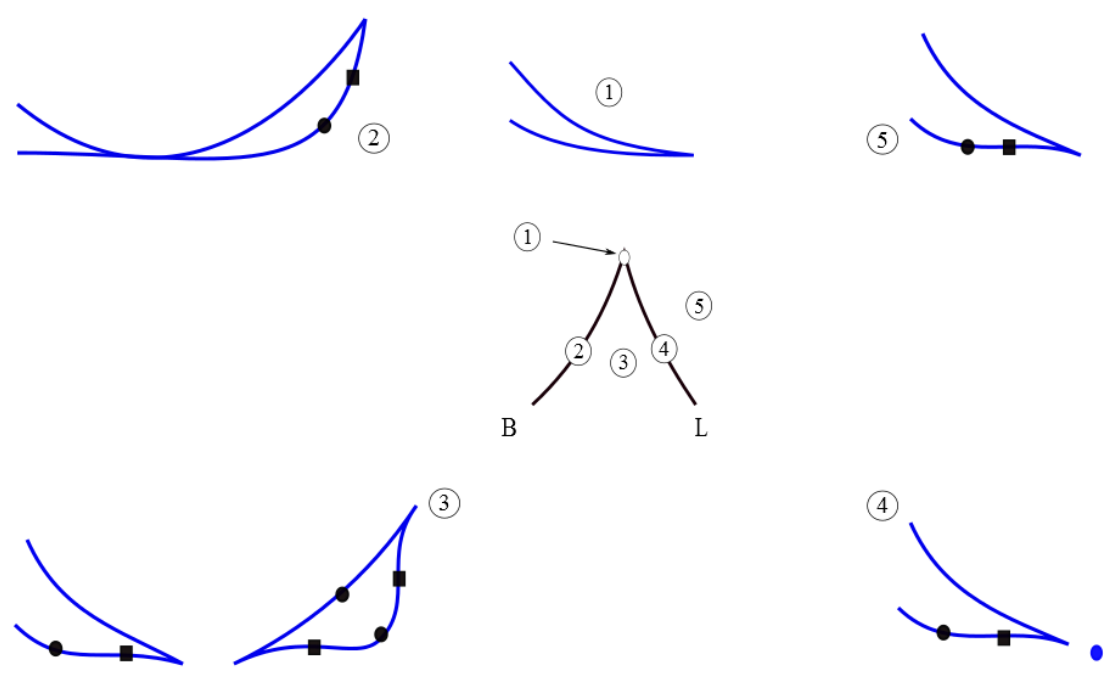

Figure 5.20 - The bifurcation of the profile at a goose singularity. The full squares represent inflection points and full discs represent vertices.

Observation 5.2 (Theorem 6.8 of [24]). For the surface $M$ let $z=f(x, y)$ be the Monge form parametrisation at the origin $p$, with $f$ as in (5.1). The family of modified orthogonal projections $\tilde{P}$ is $\mathcal{A}_{e}$-versal at a gulls singularity if and only if $a_{32}^{2}-4 a_{20} a_{44} \neq 0$.

Remark 5.5. The critical set of the projection map $\tilde{P}$ is given by

$$
\Sigma\left(\tilde{P}_{w}\right)=\left\{v+2 a_{32} x y+a_{31} x^{2}-2 a_{20} x u+O(3)=0\right\}
$$

which has an $A_{1}^{-}$-singularity at $(u, v)=(0,0)$ since $a_{32} \neq 0$.

To draw the bifurcation of the profile at a gulls singularity, we need to find all the possible strata in the bifurcation of the profile. But before doing this we devote the next section to answer the following question:

Question: When the profile at a gulls singularity has second order vertex?

\subsection{1 $A_{4}$-contact of surface with cylinder at the cusp of Gauss point}

As seen in Theorem 5.2 (ii), the profile has a second order vertex at $\tilde{P}(p)$ if and only if the surface $M$ has an $A_{4}$-contact with cylinder at a non-parabolic point $p \in M$. A gulls singularity happens at the cusp of Gauss point, which we can consider this as the origin. Therefore we consider the $A_{4}$-contact of $M$ with cylinder at a point $q=\left(x_{0}, y_{0}, f\left(x_{0}, y_{0}\right)\right)$ near the origin. According to $\$ 5.1 .1$ the Monge form at this point is given by $\tilde{\phi}=\left(x, y, g\left(x, y, x_{0}, y_{0}\right)\right)$ with $g$ as in (5.6).

Consider the vector $w=\left(w_{1}, w_{2}, w_{3}\right)$ in the orthonormal frame $\tilde{e}$ as mentioned in (5.3) and let $\mathbf{a}=\left(a_{1}, a_{2}, a_{3}\right)$ be a point in the plane $T_{w} S^{2}$. Then by using [17] the contact of $M$ with cylinder 

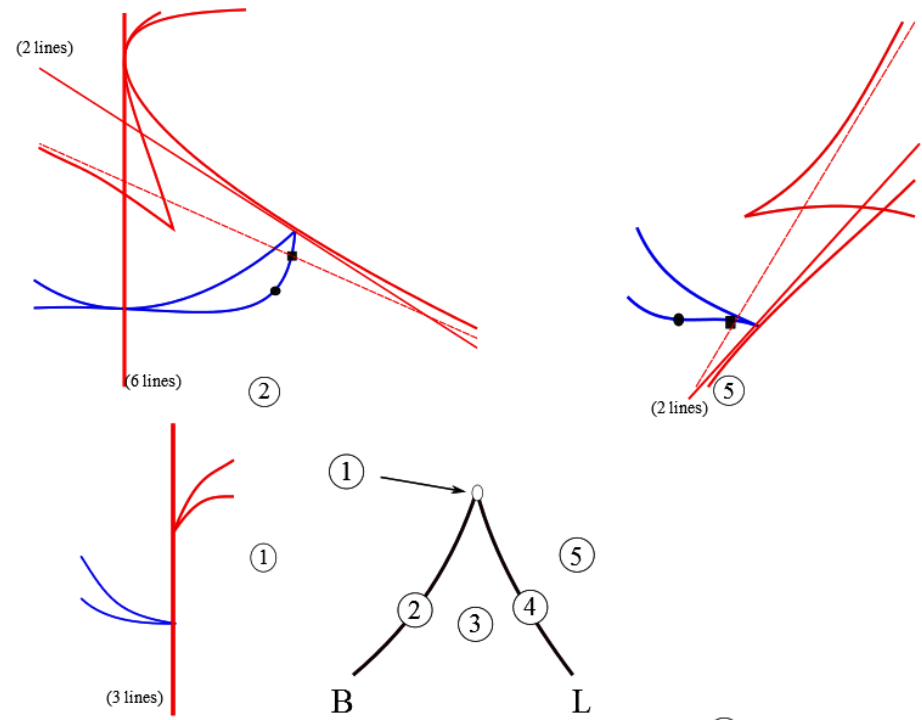

(3)
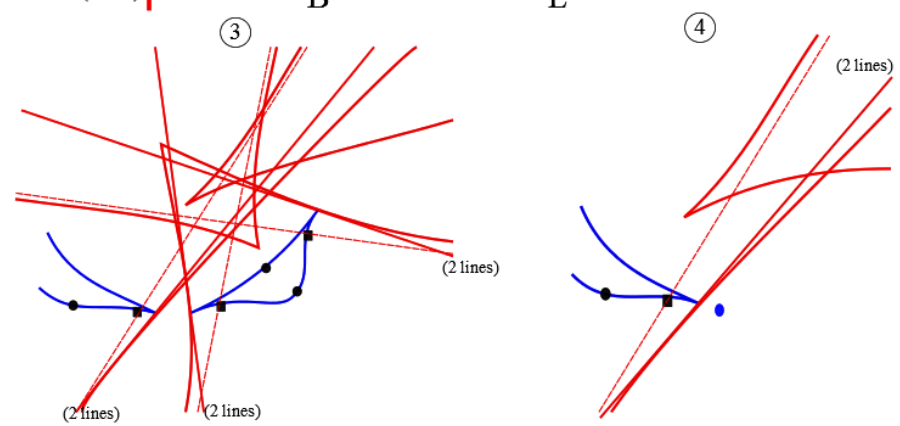

Figure 5.21 - The bifurcation of the evolute of the profile at a goose singularity.

measured by singularities of the function

$$
\psi=\langle\tilde{\phi}-\langle\tilde{\phi}, w\rangle w-\mathbf{a}, \tilde{\phi}-\langle\tilde{\phi}, w\rangle w-\mathbf{a}\rangle
$$

The contact map (5.42) has an $A_{\geq 1}$-singularity at origin if and only if $w_{3}=0, a_{1}=a_{2}=0$ and $a_{3}=\lambda$ for a non-zero real number $\lambda$. Furthermore, $\psi$ has an $A_{\geq 2}$-singularity if and only if the determinant of Hessian of $\psi$ at the origin is zero. This implies that $\lambda=\frac{2\left(A_{20} w_{1}^{2}+A_{21} w_{1} w_{2}+A_{22} w_{2}^{2}\right)}{4 A_{20} A_{22}-A_{21}^{2}}$. By Theorem 4.1 of [18], there exist a non-zero vector $(\eta, \xi)$ and a non-zero real number $s$ such that

$$
\left[\begin{array}{cc}
\frac{\partial^{2} \psi}{\partial x^{2}}(0,0) & \frac{\partial^{2} \psi}{\partial x \partial y}(0,0) \\
\frac{\partial^{2} \psi}{\partial x \partial y}(0,0) & \frac{\partial^{2} \psi}{\partial y^{2}}(0,0)
\end{array}\right]=s\left[\begin{array}{cc}
\eta^{2} & -\eta \xi \\
-\eta \xi & \xi^{2}
\end{array}\right]
$$

A straightforward calculation shows that $s=\frac{2}{A_{21}^{2}-4 A_{20} A_{22}}, \xi=-\left(A_{21} w_{1}+2 A_{22} w_{2}\right)$ and $\eta=2 A_{20} w_{1}+A_{21} w_{2}$. The contact map (5.42) has an $A_{\geq 3}$-singularity if and only if $\psi_{3}(\xi, \eta)=0$, where $\psi_{3}$ is the cubic part of $\psi$ (see Theorem 4.1 of [18]). This implies that

$$
\frac{4\left(A_{20} w_{1}^{2}+A_{21} w_{1} w_{2}+A_{22} w_{2}^{2}\right)}{4 A_{20} A_{22}-A_{21}^{2}} Q_{1}\left(x_{0}, y_{0}, w_{1}, w_{2}\right)=0
$$


with

$$
\begin{aligned}
Q_{1}\left(x_{0}, y_{0}, w_{1}, w_{2}\right)= & \left(-A_{21}^{3} A_{30}+2 A_{20} A_{21}^{2} A_{31}-4 A_{20}^{2} A_{21} A_{32}+8 A_{20}^{3} A_{33}\right) w_{1}^{3} \\
& +\left(-6 A_{21} A_{22} A_{30}+\cdots\right) w_{1}^{2} w_{2}+\left(-12 A_{21} A_{22}^{2} A_{30}+\cdots\right) w_{1} w_{2}^{2} \\
& +\left(-8 A_{22}^{3} A_{30}+\cdots\right) w_{2}^{3}+O(4) .
\end{aligned}
$$

We know that if $a_{21}=a_{22}=a_{33}=0$ and $\left(w_{1}, w_{2}\right)=(0,1)$, that is $(x, y)=(0,0)$ is cusp of Gauss and $w$ is asymptotic, then $Q_{1}(0,0,0,1)=0$. This means that $\psi$ has an $A_{\geq 3}$-singularity. Therefore at the cusp of Gauss we can consider $Q_{1}$ to obtain the $A_{\geq 3}$-contact of $M$ with cylinder.

According to Theorem 4.1 of [18] the contact map (5.42) has an $A_{\geq 4}$-singularity if and only if

$$
8 s \psi_{4}(\xi, \eta)+\left|\begin{array}{ccc}
\eta^{2} & -\xi \eta & \xi^{2} \\
\frac{\partial^{3} \psi}{\partial x^{3}}(0,0) & \frac{\partial^{3} \psi}{\partial x^{2} \partial y}(0,0) & \frac{\partial^{3} \psi}{\partial y^{2} \partial x}(0,0) \\
\frac{\partial^{3} \psi}{\partial x^{2} \partial y}(0,0) & \frac{\partial^{3} \psi}{\partial y^{2} \partial x}(0,0) & \frac{\partial^{3} \psi}{\partial y^{3}}(0,0)
\end{array}\right|=0
$$

where $\psi_{4}$ denotes the quartic part of $\psi$. By a direct calculation we get

$$
\frac{16\left(A_{20} w_{1}^{2}+A_{21} w_{1} w_{2}+A_{22} w_{2}^{2}\right)}{\left(4 A_{20} A_{22}-A_{21}^{2}\right)^{2}} Q_{2}\left(x_{0}, y_{0}, w_{1}, w_{2}\right)=0 .
$$

At the cusp of Gauss, we have $Q_{2}(0,0,0,1)=0$. So we can use the polynomial $Q_{2}$ to obtain the $A_{\geq 4}$-contact.

Now using the parametrization $w_{1}=\sin (t), w_{2}=\cos (t)$ and putting $x_{0}=x, y_{0}=y$ for simplicity, we get the system of equations

$$
\left\{\begin{array}{l}
Q_{1}(x, y, t)=0 \\
Q_{2}(x, y, t)=0
\end{array}\right.
$$

such that

$$
\begin{aligned}
Q_{1}(x, y, t)= & a_{20}^{2} a_{32}^{2} x t+a_{20} a_{31} a_{32}^{3} x^{2} t+a_{30} a_{32}^{3} x^{3}+2 a_{20} a_{32}^{3} x y t \\
& +a_{31} a_{32}^{3} x^{2} y+a_{32}^{4} x y^{2}+O(4), \\
Q_{2}(x, y, t)= & a_{21}^{2} a_{32}^{2} x t+a_{20} a_{31} a_{32}^{3} x^{2} t+a_{31}^{2} a_{32}^{3} x^{3}-3 a_{30} a_{32}^{4} x^{3} \\
& +2 a_{20} a_{32}^{4} x y t+a_{31} a_{32}^{4} x^{2} y+a_{32}^{5} x y^{2}+O(4) .
\end{aligned}
$$

Using the change of coordinate $y \mapsto y-\frac{a_{31}}{2 a_{32}} x-\frac{a_{20}}{a_{32}} t$ we get

$$
\begin{aligned}
& Q_{1}(x, y, t)=-\frac{1}{4} a_{32}^{2} x\left(\left(a_{31}^{2}-4 a_{30} a_{32}\right) x^{2}-4 a_{32}^{2} y^{2}\right)+O(4)=0, \\
& Q_{2}(x, y, t)=\frac{1}{4} a_{32}^{3} x\left(3\left(a_{31}^{2}-4 a_{30} a_{32}\right) x^{2}+4 a_{32}^{2} y^{2}\right)+O(4)=0 .
\end{aligned}
$$

The common root of the system of equations (5.44) is $x=0$. Therefore locally we can write $x$ as a function of $y$ and $t$ such that $x=c_{20} y^{2}+c_{21} y t+c_{22} t^{2}+O(3)$. By a straightforward 
calculation we obtain

$$
\begin{aligned}
& c_{20}=\frac{-2 a_{44}}{a_{32}}, \\
& c_{21}=\frac{4 a_{20} a_{44}-a_{32}^{2}}{a_{32}^{2}}, \\
& c_{22}=0 \\
& c_{30}=\frac{2 a_{44}\left(4 a_{31} a_{44}-a_{32} a_{43}\right)}{a_{32}^{3}}, \\
& c_{31}=\frac{64 a_{20} a_{31} a_{44}^{2}+20 a_{20} a_{32}^{2} a_{55}-44 a_{20} a_{32} a_{43} a_{44}+4 a_{31} a_{32}^{2} a_{44}+a_{32}^{3} a_{43}}{2 a_{32}^{4}}, \\
& c_{32}=\frac{4 a_{20}^{2} a_{43} a_{44}-4 a_{20} a_{31} a_{32} a_{44}-a_{20} a_{32}^{2} a_{43}+a_{31} a_{32}^{3}}{a_{32}^{4}}, \\
& c_{33}=0 .
\end{aligned}
$$

Substituting $x=c_{20} y^{2}+c_{21} y t+c_{22} t^{2}+O(3)$ in $Q_{2}(x, y, t)$ and after performing some suitable change of coordinates, we get $y=-\frac{a_{20}}{a_{32}} t-\frac{3\left(a_{20}^{2} a_{32} a_{43}-4 a_{20}^{2} a_{31} a_{44}\right)}{2 a_{32}^{4}} t^{2}+O(3)$. Therefore the parametrization of the $A_{4}$-curve is given by

$$
\left\{\begin{array}{l}
x=\frac{a_{20}\left(a_{32}^{2}-6 a_{20} a_{44}\right)}{a_{32}^{3}} t^{2}+x_{3} t^{3}+O\left(t^{4}\right) \\
y=-\frac{a_{20}}{a_{32}} t+y_{2} t^{2}+O\left(t^{3}\right)
\end{array}\right.
$$

where

$$
\begin{aligned}
x_{3} & =\frac{a_{20}\left(48 a_{20}^{2} a_{31} a_{44}^{2}+20 a_{20}^{2} a_{32}^{2} a_{55}-48 a_{20}^{2} a_{32} a_{43} a_{44}+12 a_{20} a_{31} a_{32}^{2} a_{44}+3 a_{20} a_{32}^{3} a_{43}-2 a_{31} a_{32}^{4}\right)}{2 a_{32}^{6}}, \\
y_{2} & =\frac{-3\left(-4 a_{20}^{2} a_{31} a_{44}+a_{20}^{2} a_{32} a_{43}\right)}{2 a_{32}^{4}} .
\end{aligned}
$$

Proposition 5.5. The $A_{4}$-curve has 4-point contact with the parabolic curve $K=0$ at the cusp of Gauss.

Proof. The parabolic curve does not depend on the choice of a Monge form and is captured by $\left(f_{x x} f_{y y}-f_{x y}^{2}\right)(x, y)$. Therefore the expression of the parabolic curve is given by

$$
\begin{aligned}
K(x, y)= & 4 a_{20} a_{32} x+\left(4 a_{20} a_{42}+12 a_{30} a_{32}-4 a_{31}^{2}\right) x^{2}+\left(12 a_{20} a_{43}-4 a_{31} a_{32}\right) y x \\
& +\left(24 a_{20} a_{44}-4 a_{32}^{2}\right) y^{2}+O(3) .
\end{aligned}
$$

If we denote the $A_{4}$-curve by $\gamma(t)=(x(t), y(t))$ with $x(t)$ and $y(t)$ as in (5.45) then

$$
F=K(\gamma(t))=\frac{-6 a_{20}^{3}\left(32 a_{20} a_{31} a_{44}^{2}-8 a_{20} a_{32} a_{43} a_{44}-4 a_{31} a_{32}^{2} a_{44}+a_{32}^{3} a_{43}\right)}{a_{32}^{5}} t^{4}+O\left(t^{5}\right) .
$$

Generically the coefficient of $t^{4}$ in $F$ is not zero. Hence $F(0)=F^{\prime}(0)=F^{\prime \prime}(0)=F^{\prime \prime \prime}(0)=0$ and $F^{(4)}(0) \neq 0$. 


\subsubsection{Stratification of the family of modified orthogonal projections at a gulls singularity}

In this part we deal with the bifurcation of the profile at a gulls singularity and study its geometry.

Proposition 5.6. Let $M$ be a surface in $\mathbb{R}^{3}$ and $\tilde{P}_{w}$ be the family of the modified orthogonal projections of a gulls singularity. Then the following strata will appear in the bifurcation of the profile of $\tilde{P}_{w}$.

i) The tacnode stratum (TC) is half a curve and is parametrised by

$$
(u, v)=\left(-\frac{\left(4 a_{31} a_{44}-a_{32} a_{43}\right)}{2 a_{20} a_{32}} t^{2}+O\left(t^{3}\right), v_{4} t^{4}+O\left(t^{5}\right)\right)
$$

where

$$
v_{4}=\frac{8 a_{20} a_{31} a_{44}^{2}-10 a_{20} a_{32}^{2} a_{55}+8 a_{20} a_{32} a_{43} a_{44}-12 a_{31} a_{32}^{2} a_{44}+3 a_{32}^{3} a_{43}}{2 a_{20} a_{32}^{2}} .
$$

ii) The parametrization of the swallowtail stratum $(S W)$ is given by

$$
(u, v)=\left(\frac{4 a_{44}}{a_{32}} t+O\left(t^{2}\right), \frac{-4 a_{44}\left(12 a_{20} a_{44}-a_{32}^{2}\right)}{a_{32}^{2}} t^{3}+O\left(t^{4}\right)\right) .
$$

iii) The beaks stratum $(B)$ is given by $(u, v)=\left(\frac{a_{32}}{a_{20}} t+O\left(t^{2}\right), \frac{3 a_{32}^{2}-4 a_{44} a_{20}}{a_{20}} t^{3}+O(4)\right)$.

iv) The second order inflection stratum (SI) consists of the origin.

v) The second vertex stratum has 3 branches such that one is a cusp and two other are tangential parabolas.

Proof. We equip the surface $M$ by the Monge form parametrisation $\phi(x, y)=(x, y, f(x, y))$ with $f$ as in (5.1).

i) We know that the stratification must become homeomorph to the $\mathcal{A}$-stratification given in page 84 of [23], therefore the tacnode stratum is half a curve. To obtain a precise parametrization for this stratum we use the fact that a tacnode happens at two different points $p_{1}=\phi\left(x_{1}, y_{1}\right)$ and $p_{2}=\phi\left(x_{2}, y_{2}\right)$ in the critical set $\Sigma\left(\tilde{P}_{w}\right)$ with $\phi\left(x_{1}, y_{1}\right)=\phi\left(x_{2}, y_{2}\right)$ and both are tangential folds. Therefore after performing the change of coordinate $x \mapsto \frac{1}{\cos (u)} x-\tan (u) y$ we may assume that $x_{1}=x_{2}=x$. Now if we denote the third component of $\phi$ by $g$, then the tacnode stratum is captured by the solution of system of equations

$$
\left\{\begin{array}{l}
g\left(x, y_{1}\right)-g\left(x, y_{2}\right)=0 \\
\frac{\partial g}{\partial y}\left(x, y_{1}\right)=0 \\
\frac{\partial g}{\partial y}\left(x, y_{2}\right)=0 \\
\frac{\partial g}{\partial x}\left(x, y_{1}\right)-\frac{\partial g}{\partial x}\left(x, y_{2}\right)=0
\end{array}\right.
$$


From the second and third equations of (5.47) we have $-2 a_{32} x+O(2)=0$. Since $a_{32} \neq 0$, by the implicit function theorem we have

$$
x=-\frac{2 a_{44}}{a_{32}} y_{1}^{2}-\frac{2 a_{44}}{a_{32}} y_{1} y_{2}+\frac{3}{2} y_{1} u-\frac{2 a_{44}}{a_{32}} y_{2}^{2}+\frac{3}{2} y_{2} u+O(3) .
$$

By substituting $x$ in the last equation of (5.47) we get $u=\frac{a_{32}}{2 a_{20}} y_{2}+\frac{a_{32}}{2 a_{20}} y_{1}+O(2)$. Finally after putting $x$ and $u$ in the first equation of (5.47) we have

$$
2 a_{20} a_{32}\left(-y_{1}+y_{2}\right)\left(y_{2}+y_{1}\right)\left(4 a_{20} a_{44}-a_{32}^{2}\right)+O(2)=0 \text {. }
$$

Since $y_{1} \neq y_{2}$, we have $y_{2}=-y_{1}+O(2)$. Also from the first or second equations of (5.47) we can find $v$ as well. Consequently, the parametrization of tacnode stratum is given by $(u, v)=$ $\left(-\frac{\left(4 a_{31} a_{44}-a_{32} a_{43}\right)}{2 a_{20} a_{32}} t^{2}+\right.$ h.o.t, $v_{4} t^{4}+$ h.o.t $)$, where

$$
v_{4}=\frac{8 a_{20} a_{31} a_{44}^{2}-10 a_{20} a_{32}^{2} a_{55}+8 a_{20} a_{32} a_{43} a_{44}-12 a_{31} a_{32}^{2} a_{44}+3 a_{32}^{3} a_{43}}{2 a_{20} a_{32}^{2}} .
$$

ii) Following the notations of part (i), the swallowtail stratum is obtained by solving the system of equations

$$
\frac{\partial g}{\partial y}=\frac{\partial^{2} g}{\partial y^{2}}=\frac{\partial^{3} g}{\partial y^{3}}=0
$$

By a direct calculation using the implicit function theorem, one can obtain the parametrization of the swallowtail stratum.

iii) The beaks stratum happens when the critical set has a singularity. To find this stratum we should consider the following system of equations:

$$
\frac{\partial g}{\partial y}=\frac{\partial^{2} g}{\partial y^{2}}=\frac{\partial^{2} g}{\partial y \partial x}=0
$$

We have $\frac{\partial^{2} g}{\partial y \partial x}=-2 a_{20} u+O(2)=0$ and hence the implicit function theorem implies that $u=\frac{a_{31}}{a_{20}} x+\frac{a_{32}}{a_{20}} y+O(2)$. After substituting $u$ in $\frac{\partial^{2} g}{\partial y^{2}}$, we will obtain $x$ as a function of $y$ and so $x=\frac{-3\left(2 a_{20} a_{44}-a_{32}^{2}\right)}{a_{20} a_{32}} y^{2}+O\left(y^{3}\right)$. Moreover, one can obtain $v$ from the equation $\frac{\partial g}{\partial y}=0$. Therefore this stratum is given by

$$
(u, v)=\left(\frac{a 32}{a 20} t+O\left(t^{2}\right), \frac{3 a_{32}^{2}-4 a_{44} a_{20}}{a_{20}} t^{3}+O(4)\right) .
$$

iv) According to part (iii) of Theorem 5.3 we know that the profile has a second order inflection when the critical set passes through the cusp of Gauss point. Therefore the second order inflection stratum only consists of the origin.

v) In $\S 5.8 .1$ we obtained the $A_{4}$-curve. Following the notations used in $\S 5.8 .1$ when we substitute $x=c_{20} y^{2}+c_{21} y t+c_{22} t^{2}+O(3)$ in $Q_{2}(x, y, t)$, it becomes $\mathcal{A}$-equivalent to $t y^{3} \pm t^{7}$. The zero set of this singularity has 2 branches given by

$$
\left\{\begin{array}{l}
t=\frac{10\left(-2 a_{32} a_{43} a_{44}+4 a_{31} a_{44}^{2}+a_{32} a_{55}\right)}{a_{32}\left(a_{32}^{2}-4 a_{20} a_{44}\right)} y^{2}+O\left(y^{3}\right), \\
t=-\frac{a_{32}}{a_{20}} y-\frac{3\left(a_{32} a_{43}-4 a_{31} a_{44}\right)}{2 a_{20} a_{32}} y^{2}+O\left(y^{3}\right) .
\end{array}\right.
$$


On the other hand if we write the vector $w$ in the fram $\left\{e_{1}, e_{2}, e_{3}\right\}$, with

$$
e_{1}=(1,0,0), \quad e_{2}=(0,1,0), \quad e_{3}=(0,0,1)
$$

then

$$
w=-\cos (v) \sin (u) e_{1}+\cos (v) \cos (u) e_{2}-\sin (v) e_{3} .
$$

Now by applying the change of basis matrix (5.4), we can write $w$ in the new orthonormal frame $\left\{\tilde{e}_{1}, \tilde{e}_{2}, \tilde{e}_{3}\right\}$ as mentioned in (5.3). Then we get

$$
w=w_{1} \tilde{e}_{1}+w_{2} \tilde{e}_{2}+w_{3} \tilde{e}_{3}
$$

with $w_{1}=\sin (t(y))$ and $w_{2}=\cos (t(y))$ where $t(y)$ is given in (5.48). The rest is a matter of using the implicit function theorem to get the second order vertex stratum parametrized by

$$
\left\{\begin{array}{l}
u=\frac{10\left(a_{32} a_{43}-4 a_{31} a_{44}\right)}{a_{32}\left(a_{32}^{2}-4 a_{20} a_{44}\right)} y^{2}+O\left(y^{3}\right) \\
v= \pm\left(\frac{a_{32}}{a_{20}} y+\frac{3\left(a_{32} a_{43}-4 a_{31} a_{44}\right)}{2 a_{20} a_{32}} y^{2}+O\left(y^{3}\right)\right),
\end{array}\right.
$$

and

$$
\left\{\begin{array}{l}
u=\frac{-\epsilon a_{32}}{a_{20}} y^{2}+O\left(y^{3}\right) \\
v= \pm \frac{\sqrt{3 \epsilon\left(a_{32} a_{43}-4 a_{31} a_{44}\right)}}{a_{20}} y^{3}+O\left(y^{4}\right)
\end{array}\right.
$$

where $\epsilon= \pm 1$ and $\epsilon\left(a_{32} a_{43}-4 a_{31} a_{44}\right)>0$.

The parametrization (5.49) gives 2 tangential parabolas and (5.50) gives a cusp.

Remark 5.6. Note that in Proposition 5.6 when $(u, v)=(0,0)$, then the expression of the vertex curve $V\left(x_{0}, y_{0}\right)=0$ is given by

$$
V\left(x_{0}, y_{0}\right)=-8 a_{30}^{3} x_{0}^{3}-8 a_{31} a_{32}^{3} x_{0}^{2} y_{0}-8 a_{32}^{4} x_{0} y_{0}^{2}+\cdots-16 a_{44} a_{32}^{3} y_{0}^{4}+O(5)=0 .
$$

This curve has a $D_{4}$-singularity. The discriminant of the cubic part of (5.51) is equal to

$$
\Delta=-a_{32}^{14}\left(4 a_{30} a_{32}-a_{31}^{2}\right) y_{0}^{6}
$$

Therefore the vertex curve (5.51) has a

$$
\left\{\begin{array}{c}
D_{4}^{+} \text {-singularity } \quad \text { if } \quad 4 a_{20} a_{32}-a_{31}^{2}>0 \\
D_{4}^{-} \text {-singularity } \quad \text { if } \quad 4 a_{20} a_{32}-a_{31}^{2}<0 .
\end{array}\right.
$$

According to Theorem 5.5, the $D_{5}$-beaks occurs at an isolated point on the parabolic set. Therefore generically around the cusp of Gauss point the vertex curve either has a $D_{4}^{+}$-singularity or a $D_{4}^{-}$-singularity. We call these two gulls singularities by $D_{4}^{+}$-gulls or $D_{4}^{-}$-gulls, respectively.

In Figure 5.23 we draw the bifurcation of the profile at a gulls singularity. Note that according to Proposition 5.6 the stratification depends on sign of coefficients of the parametrization of each stratum. There are many possibilities, but one can observe that all stratifications are homeomorph. 


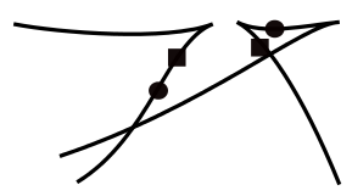

Figure 5.22 - The position of inflections and vertices in the curve (23) of Figure 5.23. Discs represent vertex points and full squares represent inflection points.

When we cross the beak-stratum (2) and (14) in Figure 5.23), by using Theorems 5.4 and 5.5 we can determine the position of inflections and vertices in the curves 23 or 13 in Figure 5.23 (see for example Figure 5.22)

Therefore, we expect to have inflection transverse stratum in the bifurcation of the profile at a gulls singularity. The inflection transverse stratum and vertex transverse stratum are captured by some complicated singularities and we leave them for future work.

The next step is to consider the geometry of the profile at a gulls singularity. To do this we must study the intersection of the curves $\tilde{K}(x, y)=0, V(x, y)=0$ and $A_{4}$-curve with the critical set $\Sigma\left(\tilde{P}_{w}\right)$ in order to find the maximum number of ordinary vertices and ordinary inflections in the bifurcation of the profile at a gulls singularity. This is a work in progress and the following conjecture is important for us.

Conjecture. In the bifurcation of the profile at a $D_{4}^{+}$-gulls singularity there are at most 15 vertices (9 vertices concentrated at 3 cusps) and 8 inflections (6 vertices concentrated at 3 cusps).

\subsection{On $F R S$-theory for discriminant of map germs $\mathbb{R}^{2} \rightarrow \mathbb{R}^{2}$}

Finding a theory that explains the deformations of the singularity of a submanifold of $\mathbb{R}^{n}$ as well as the changes in its geometry is still an open problem. In Chapter 2 we introduce a way to answer this problem for family of plane curves with $\mathcal{A}_{e}$-codimension $\leq 2$. Following to what we defined for families of plane curves, we can extend the definition of $F R S$-equivalence for discriminant of map germs $f: \mathbb{R}^{2} \rightarrow \mathbb{R}^{2}$.

Definition 5.4. Two germs of m-parameter deformations $F_{s}$ and $G_{u}$ of a map germ $f: \mathbb{R}^{2} \rightarrow \mathbb{R}^{2}$ are FRS-equivalent if there exists a germ of a homeomorphism

$$
k:\left(\mathbb{R}^{m},\left(S_{1}, 0\right)\right) \rightarrow\left(\mathbb{R}^{m},\left(S_{2}, 0\right)\right),
$$

where $S_{1}$ and $S_{2}$ are stratifications of the parameter spaces such that

(i) $F_{s}$ is diffeomorphic to $G_{k(s)}$ in each stratum of $S_{1}$;

(ii) $\Delta\left(F_{s}\right)$ and $\Delta\left(G_{k(s)}\right)$ have the same number of inflections and vertices in each stratum;

(iii) the relative position of the singularities, points of self-intersections, inflections and vertices on $\Delta\left(F_{s}\right)$ and $\Delta\left(G_{k(s)}\right)$ is the same in each stratum. 

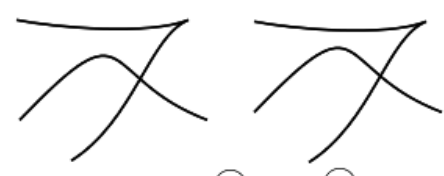

(16)
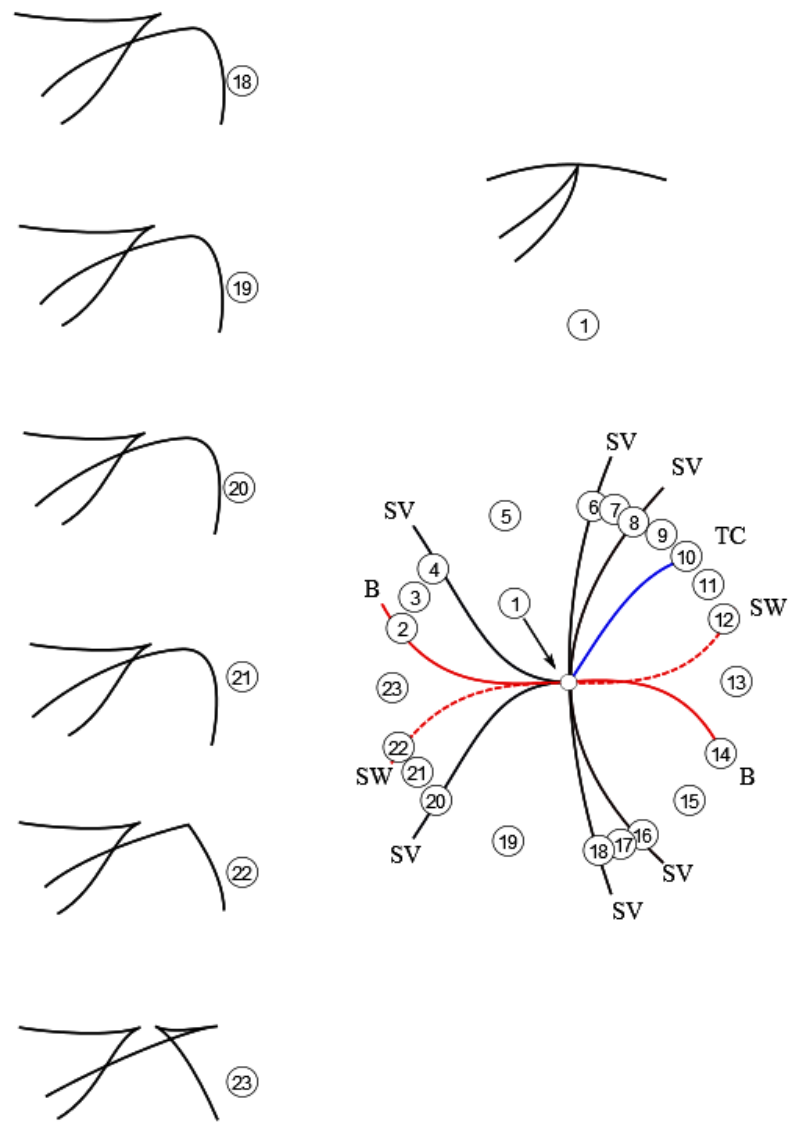

(10)

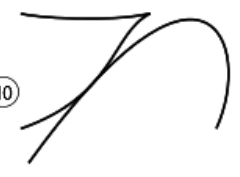

(9)

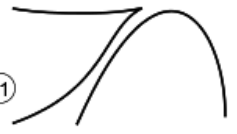

(1)

(14)

(13)

(12)
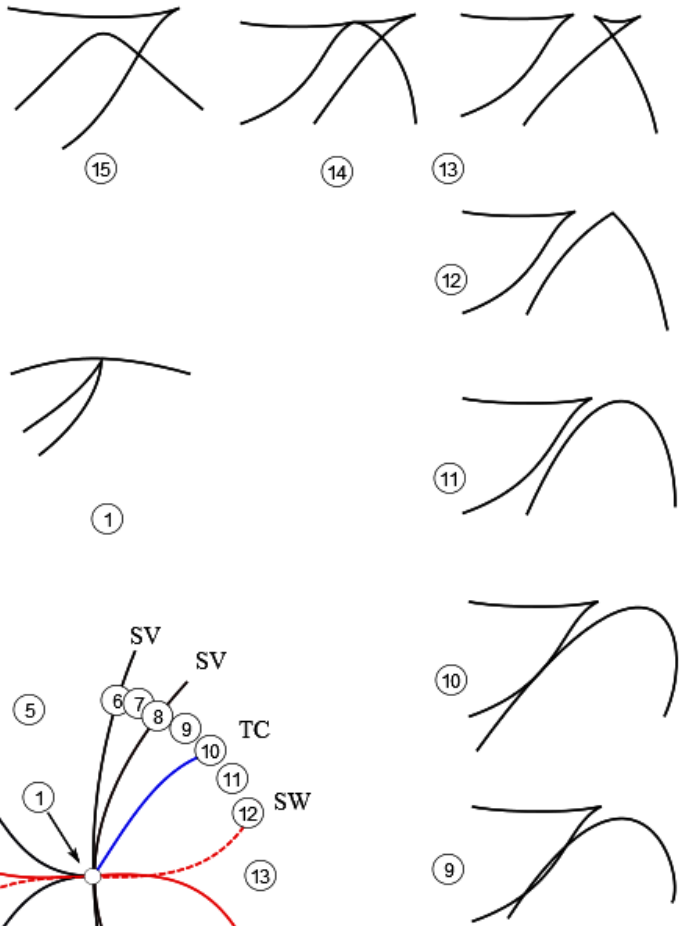

(8)

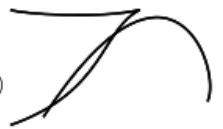

(7)

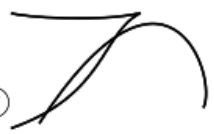

(2)
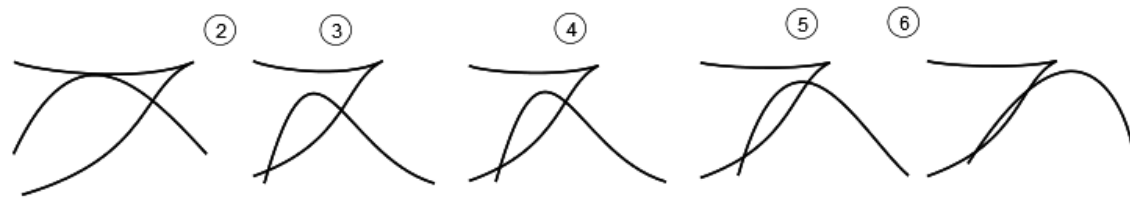

Figure 5.23 - The bifurcation of the profile at a gulls singularity.

Here $\Delta(F)=F(\Sigma(F))$ is the discriminant of $F$.

According to what we observed in the geometry of the profiles in this chapter we can obtain $F R S$-model for the discriminant of a map germ $\mathbb{R}^{2} \rightarrow \mathbb{R}^{2}$ with $\mathcal{A}_{e}$-codimension $\leq 2$. For example at a $D_{4}^{+}$-beaks singularity, we found some conditions on the coefficients of the Monge form. With these conditions we can construct an $F R S$-model case by case.

Finally, a family of a map-germ $\mathbb{R}^{2} \rightarrow \mathbb{R}^{2}$ with $\mathcal{A}_{e}$-codimension $\leq 2$ is $F R S$-generic if it is $F R S$-equivalent to the corresponding $F R S$-model family. 
[1] V. I. Arnold, Geometrical methods in the theory of ordinary differential equations. SpringerVerlag, New York-Berlin, 1983.

[2] V. I. Arnold, Wave front evolution and equivariant Morse Lemma. Comm. Pure Appl. Math. 29 (1976), 557-582.

[3] V. I. Arnold, S. M. Gusel̆n-Zade and A. N. Varchenko, Singularities of differentiable maps. Vol. I. The classification of critical points, caustics and wave fronts. Monographs in Mathematics, 82. Birkhäuser Boston, Inc., Boston, MA, 1985.

[4] J. W. Bruce, The dual of generic hypersurfaces. Math. Scand. 49 (1981), 36-60.

[5] J. W. Bruce, Wavefronts and parallels in Euclidean space. Math. Proc. Camb. Phil. Soc. 93 (1983), 323-333.

[6] J. W. Bruce and P. J. Giblin, Curves and singularities. Cambridge University Press, Cambridge, 1984.

[7] J. W. Bruce and P. J. Giblin, Smooth stable maps of discriminant varieties. Proc. Lond. Math. Soc. 50 (1985), 535-551.

[8] J. W. Bruce and J. M. West, Functions on a crosscap. Math. Proc. Camb. Phil. Soc. 123 (1998), 19-39.

[9] Th. Broker and L. Lander, Differentiable Germs and Catastrophes, Cambridge University Press, 1975.

[10] G. Capitanio and A. Diatta, Perestroikas of vertex sets at umbilic points. Funct. Anal. Other Math. 2 (2009), 165-178.

[11] S-N. Chow, C. Li and D. Wang, Normal forms and bifurcation of planar vector fields. Cambridge University Press, Cambridge, 1994.

[12] J. M. S. David, Projection-generic curves. J. London Math. Soc. 27 (1983), 552-562.

[13] F. S. Dias and F. Tari, On vertices and inflections of plane curves. Preprint, 2015.

[14] F. S. Dias and J. J. Nuño Ballesteros, Plane curve diagrams and geometrical applications. Q. J. Math. 59 (2008), 287-310. 
[15] A. Diatta and P. Giblin, Vertices and inflexions of plane sections of surfaces in $\mathbb{R}^{3}$. Real and complex singularities, 71-97, Trends Math., Birkhäuser, 2007.

[16] F. Dumortier, Techniques in the theory of local bifurcations: blow-up, normal forms, nilpotent bifurcations, singular perturbations. Bifurcations and periodic orbits of vector fields (Montreal, PQ, 1992), 19-73, NATO Adv. Sci. Inst. Ser. C Math. Phys. Sci., 408, Kluwer Acad. Publ., Dordrecht, 1993.

[17] T. Fukui, M. Hasegawa and K. Nakagawa, Contact of a regular surface in Euclidian 3-space with cylinder and cubic binary differential equations. Preprint, 2014.

[18] T. Fukui, M. Hasegawa, Singularities of paralel surfaces. Tohoku Math. J. 64 (2012), 383-408.

[19] T. Fukunaga and M. Takahashi, Evolutes of fronts in the Euclidean plane. Journal of Singularities 10 (2014), 92-107.

[20] T. Gaffney, The structure of the $T \mathcal{A}(f)$, classification and application to differential geometry. Singularities, part 1 (Arcata, Calif., 1981), Proc. Sympos. Pure Math. 40, Amer. Math. Soc, Providence, RI, (1983), 409-427.

[21] C. G. Gibson and C. A. Hobbs, Singularities of general one-dimensional motions of the plane and space. Proc. Roy. Soc. Edinburgh Sect. A 125 (1995), 639-656.

[22] V. V. Goryunov, Projections of Generic Surfaces with Boundaries. Adv. Soviet Math. 1 (1990), 157-200.

[23] W. Hawes, Multi-dimensional Motions of the Plane and Space. Ph.D. thesis, university of Liverpool, 1994.

[24] S. Izumiya, M. C. Romero Fuster, M. A. S. Ruas and F. Tari, Differential Geometry from a Singularity Theory Viewpoint. World Scientific Publishing Company, 2015.

[25] J. A. Montaldi, On contact between submanifolds. Michigan Math. J. 33, (1986) 81-85.

[26] R. Oset Sinha and F. Tari, Projections of space curves and duality. Q. J. Math 64 (2013), 281-302.

[27] R. Roussarie, Bifurcations of planar vector fields and Hilbert's 16th Problem. Publications of the 20th Brazilian Mathematical Colloquium, IMPA, 1995.

[28] F. Tari, Two-parameter families of binary differential equations. Discrete Contin. Dyn. Syst. 22 (2008), 759-789.

[29] F. Tari, Geometric properties of the solutions of implicit differential equations. Discrete Contin. Dyn. Syst. 17 (2007), 349-364. 
[30] F. Tari, Two-parameter families of implicit differential equations. Discrete Contin. Dyn. Syst. 13 (2005), 139-162.

[31] R. Uribe-Vargas, On singularities, "perestroikas" and differential geometry of space curves. Enseign. Math. 50 (2004), 69-101.

[32] C. T. C. Wall, Flat singularity theory. J. London. Math. Soc. 87 (2013), 622-640.

[33] C. T. C. Wall, Finite determinacy of smooth map-germs. Bull. London. Math. Soc. 13 (1981), 481-539.

[34] C. T. C. Wall, Geometric properties of generic differentiable manifolds. Lecture Notes in Math. 597 (1977), 707-774. 

APPENDIX

\section{A}

\section{MAPLE CODES IN THE PROOF OF THEOREM 4.3}

Mapel codes in the general proof of Theorem 4.3. Here K, L, T and P represent $\kappa^{\prime}, \kappa^{\prime \prime}, \tau^{\prime}$ and $\tau^{\prime \prime}$ respectively.

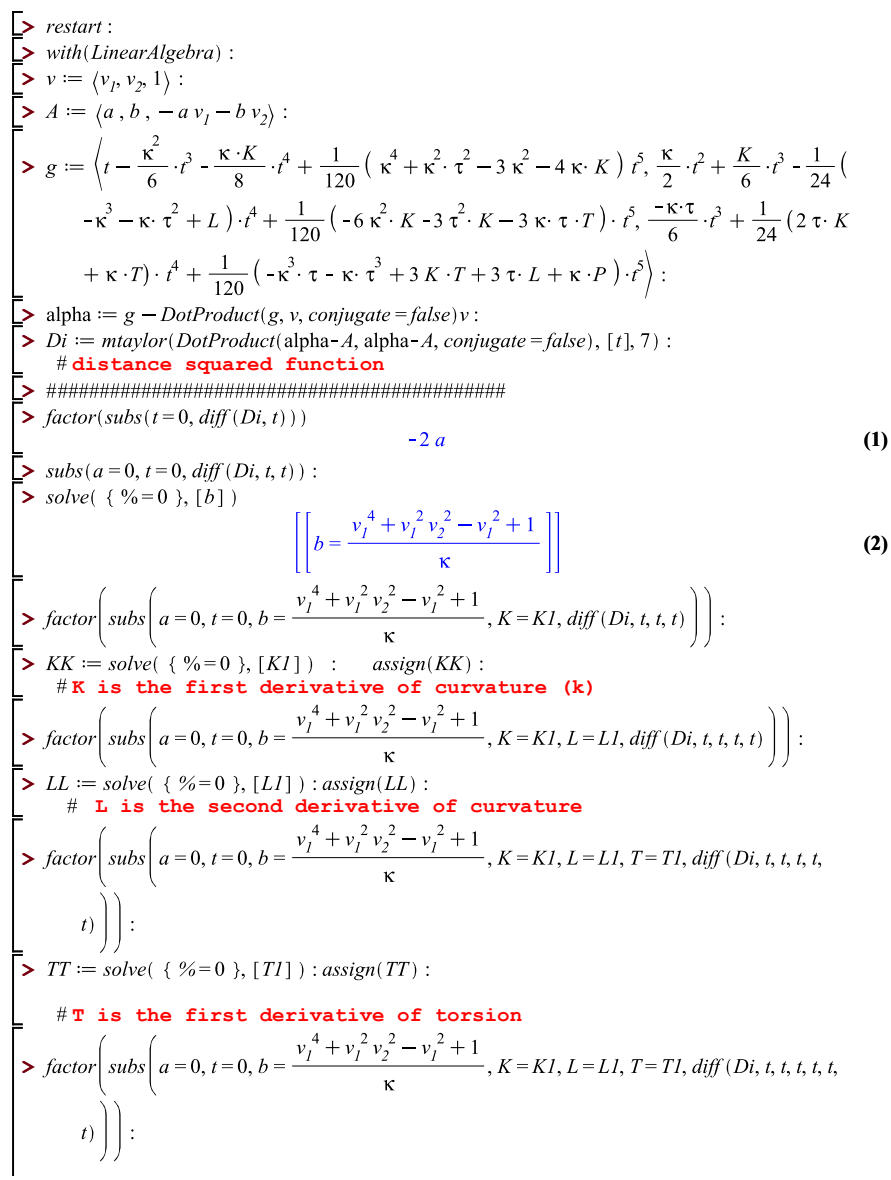




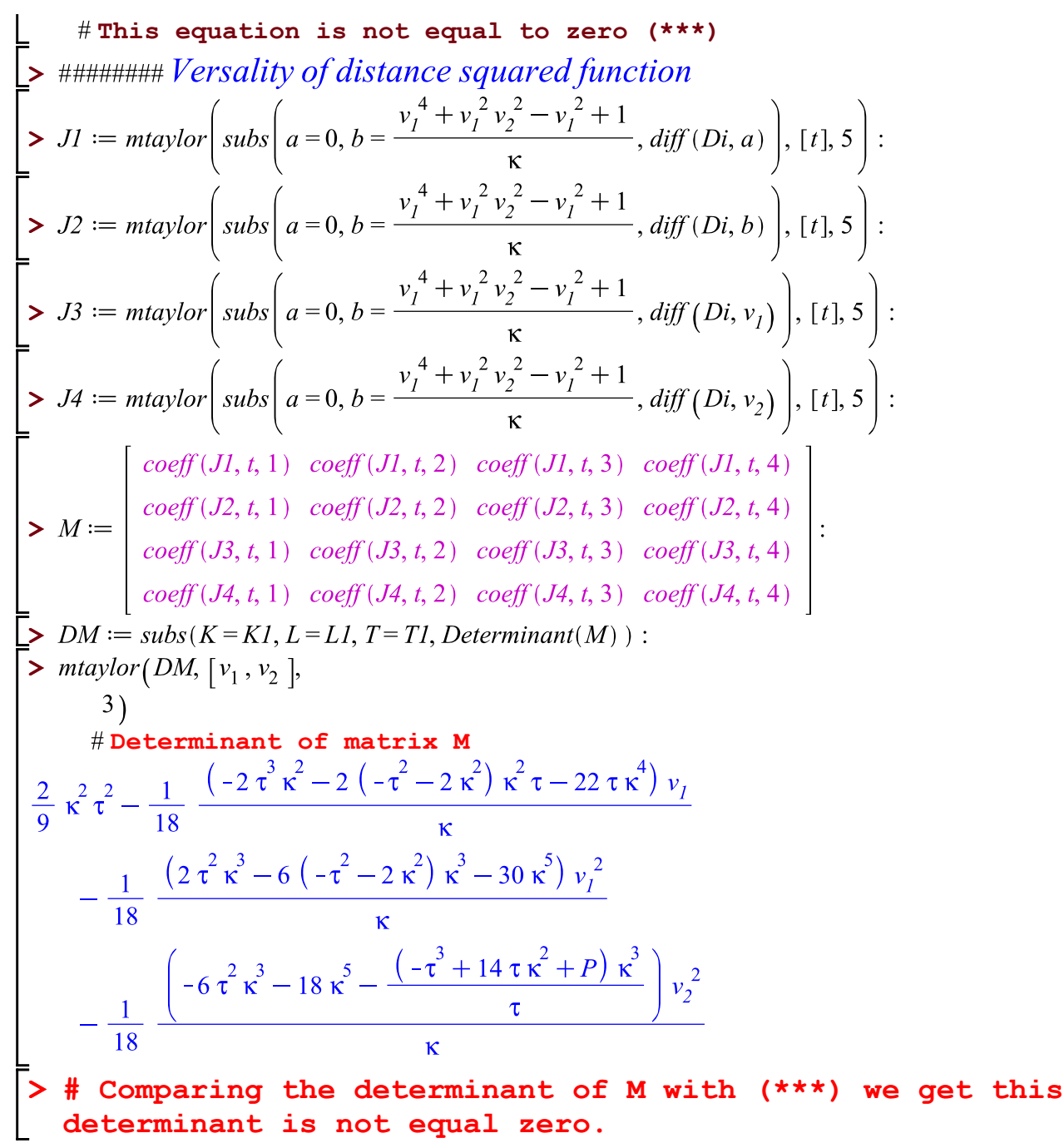


APPENDIX

\section{MAPLE CODES IN THE PROOF OF THEOREM 4.4}

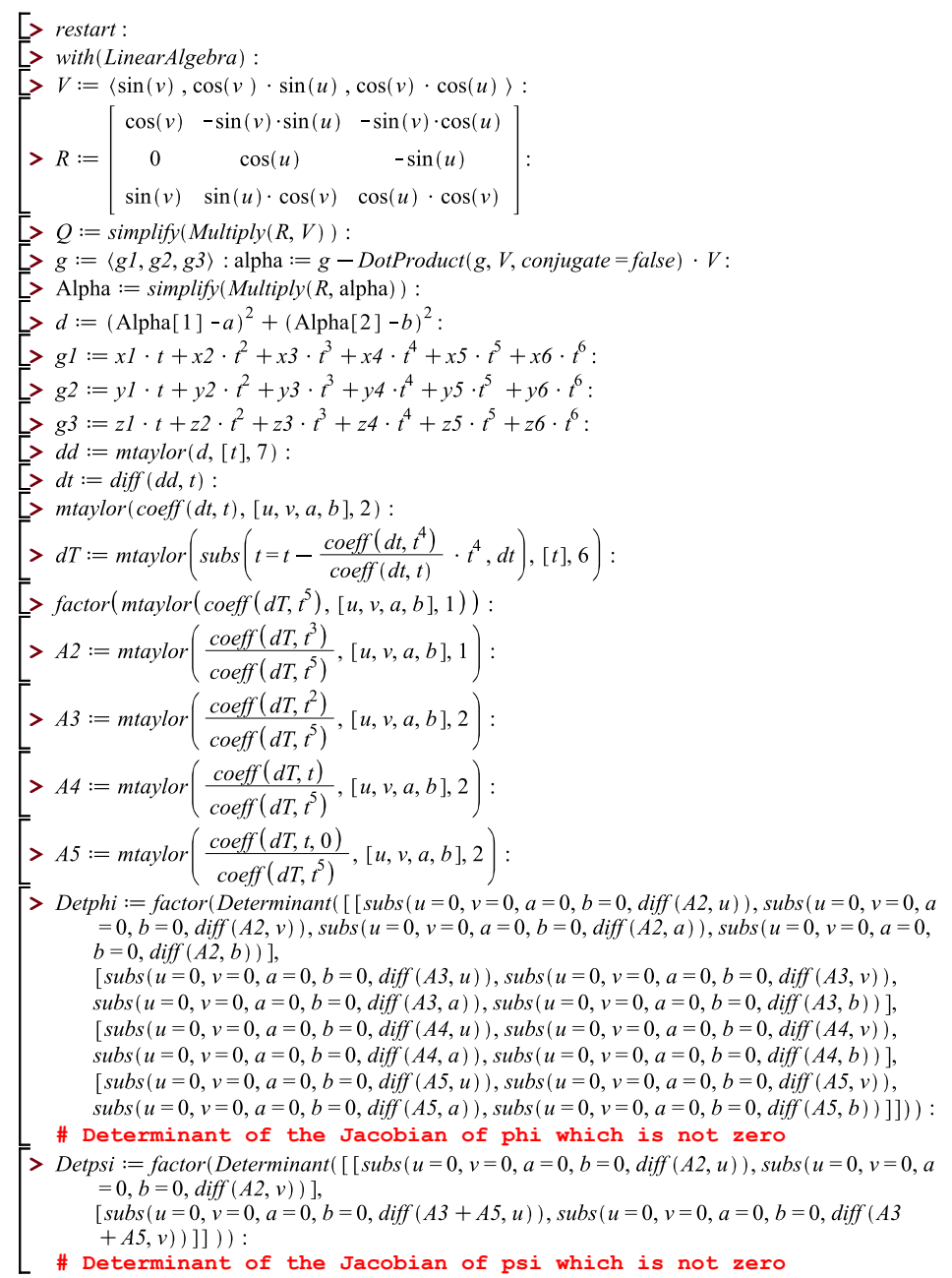



APPENDIX

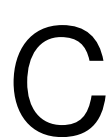

\section{MAPLE CODES IN THE PROOF OF THEOREM 5.4}

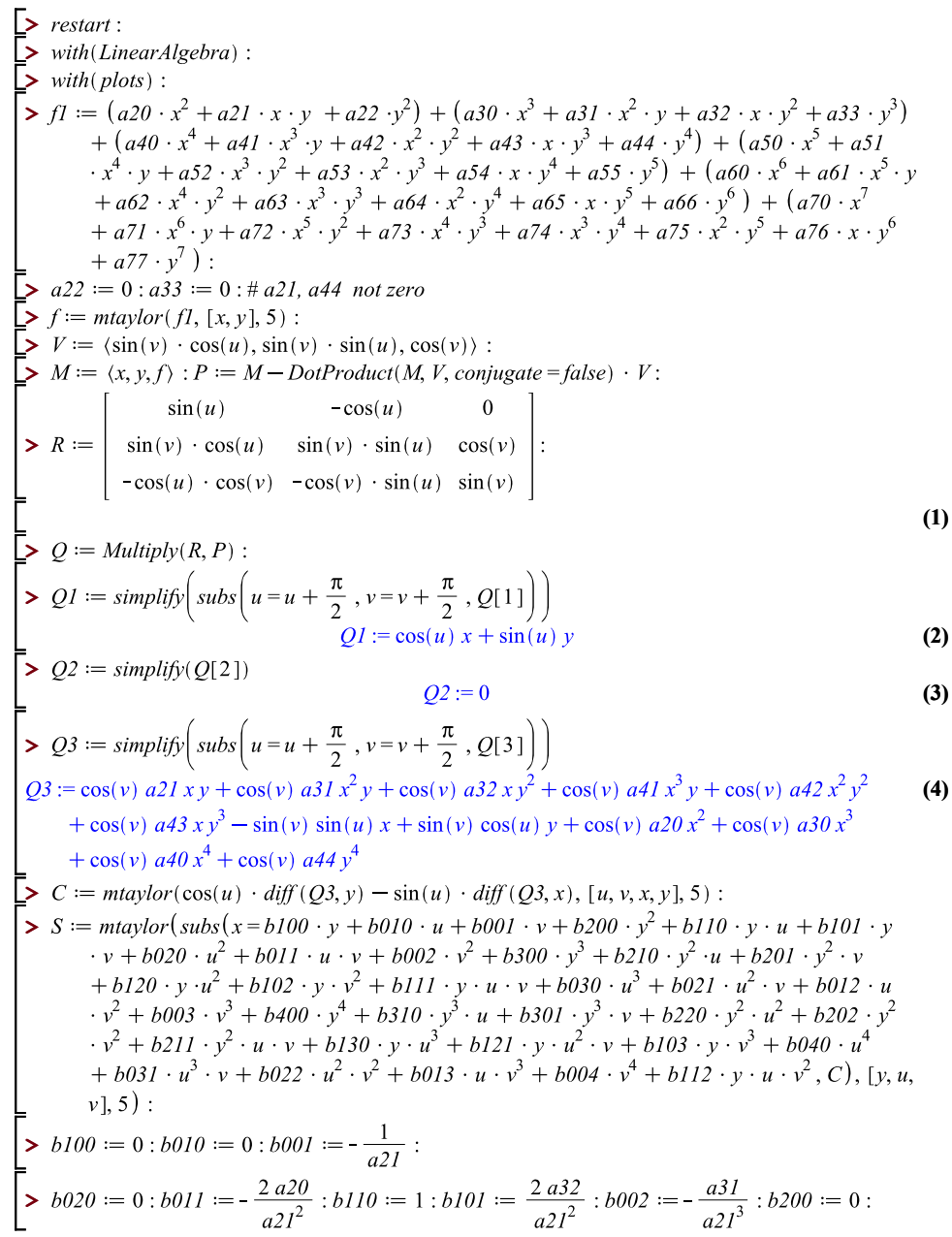




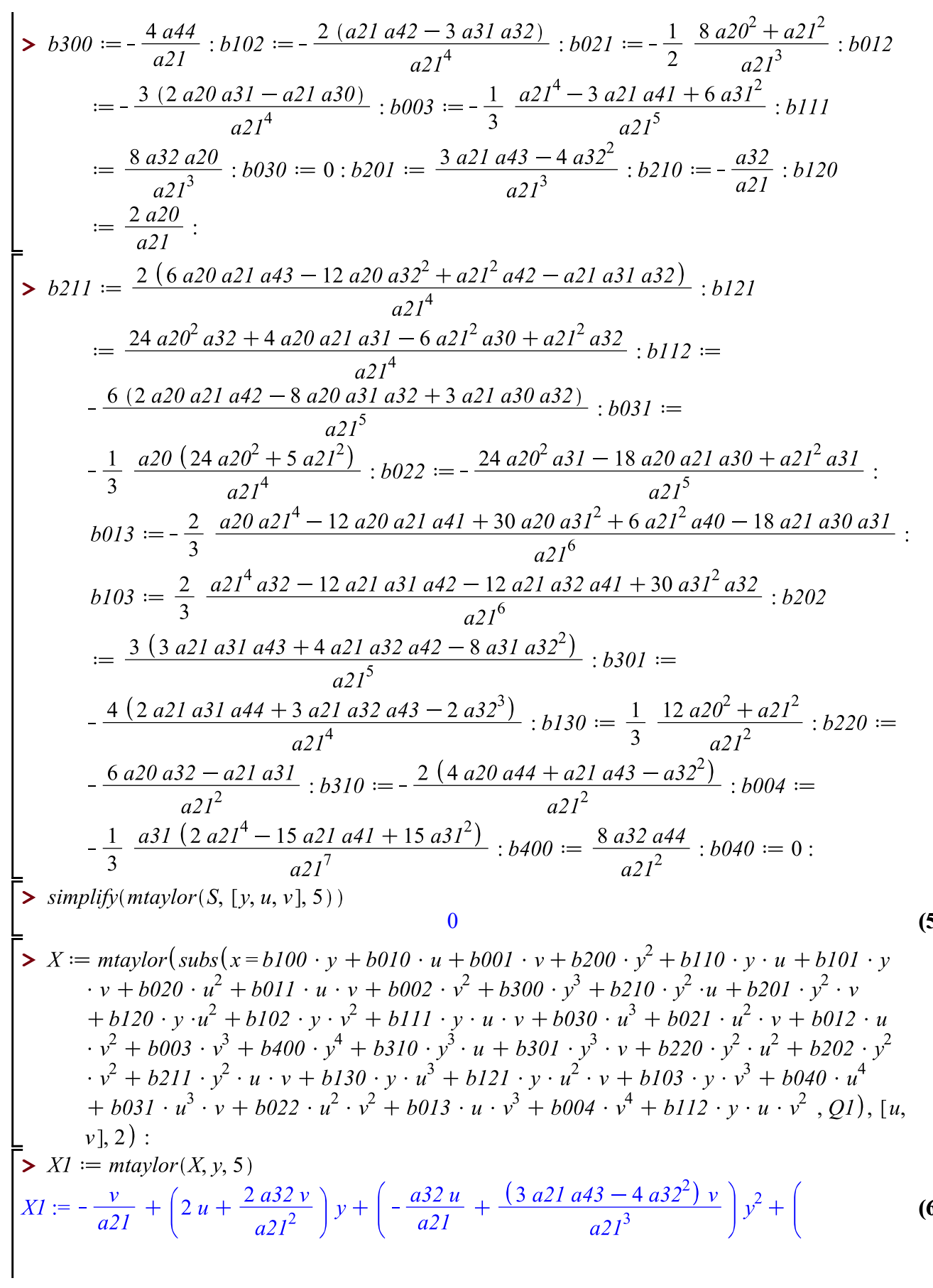


$-\frac{2\left(4 a 20 a 44+a 21 a 43-a 32^{2}\right) u}{a 21^{2}}-\frac{4\left(2 a 21 a 31 a 44+3 a 21 a 32 a 43-2 a 32^{3}\right) v}{a 21^{4}}$

$\left.-\frac{4 a 44}{a 21}\right) y^{3}+\frac{8 a 32 a 44 y^{4}}{a 21^{2}}$

$Y:=$ mtaylor $\left(\operatorname{subs}\left(x=b 100 \cdot y+b 010 \cdot u+b 001 \cdot v+b 200 \cdot y^{2}+b 110 \cdot y \cdot u+b 101 \cdot y\right.\right.$

$\cdot v+b 020 \cdot u^{2}+b 011 \cdot u \cdot v+b 002 \cdot v^{2}+b 300 \cdot y^{3}+b 210 \cdot y^{2} \cdot u+b 201 \cdot y^{2} \cdot v$

$+b 120 \cdot y \cdot u^{2}+b 102 \cdot y \cdot v^{2}+b 111 \cdot y \cdot u \cdot v+b 030 \cdot u^{3}+b 021 \cdot u^{2} \cdot v+b 012 \cdot u$

$\cdot v^{2}+b 003 \cdot v^{3}+b 400 \cdot y^{4}+b 310 \cdot y^{3} \cdot u+b 301 \cdot y^{3} \cdot v+b 220 \cdot y^{2} \cdot u^{2}+b 202 \cdot y^{2}$

$\cdot v^{2}+b 211 \cdot y^{2} \cdot u \cdot v+b 130 \cdot y \cdot u^{3}+b 121 \cdot y \cdot u^{2} \cdot v+b 103 \cdot y \cdot v^{3}+b 040 \cdot u^{4}$

$\left.+b 031 \cdot u^{3} \cdot v+b 022 \cdot u^{2} \cdot v^{2}+b 013 \cdot u \cdot v^{3}+b 004 \cdot v^{4}+b 112 \cdot y \cdot u \cdot v^{2}, Q 3\right),[u$, $v], 2)$ :

$Y 1:=$ mtaylor $(Y, y, 6)$

$Y 1:=\left(a 21 u+\frac{a 32 v}{a 21}\right) y^{2}+\left(\frac{3 a 21 a 43-4 a 32^{2}}{a 21^{2}}+\frac{2 a 32^{2}}{a 21^{2}}+\frac{8 a 20 a 44}{a 21^{2}}-\frac{a 43}{a 21}\right) v y^{3}$

$+\left(-3 a 44+\left(-\frac{4\left(2 a 21 a 31 a 44+3 a 21 a 32 a 43-2 a 32^{3}\right)}{a 21^{3}}\right.\right.$

$\left.+\frac{a 32\left(3 a 21 a 43-4 a 32^{2}\right)}{a 21^{3}}-\frac{32 a 20 a 44 a 32}{a 21^{3}}+\frac{8 a 31 a 44}{a 21^{2}}+\frac{2 a 43 a 32}{a 21^{2}}\right) v+($

$\left.\left.-\frac{2\left(4 a 20 a 44+a 21 a 43-a 32^{2}\right)}{a 21}-\frac{8 a 20 a 44}{a 21}+a 43-\frac{a 32^{2}}{a 21}\right) u\right) y^{4}+(($

$\frac{4 a 32\left(2 a 21 a 31 a 44+3 a 21 a 32 a 43-2 a 32^{3}\right)}{4}-\frac{8 a 20 a 44\left(3 a 21 a 43-4 a 32^{2}\right)}{4}$

$$
\text { a2 } 1^{4} \quad a 21^{4}
$$

$\left.+\frac{32 a 20 a 32^{2} a 44}{a 21^{4}}+\frac{a 43\left(3 a 21 a 43-4 a 32^{2}\right)}{a 21^{3}}-\frac{32 a 31 a 44 a 32}{a 21^{3}}+\frac{8 a 42 a 44}{a 21^{2}}\right) v$

$+\frac{4 a 32 a 44}{a 21}+\left(\frac{24 a 20 a 44 a 32}{a 21^{2}}-\frac{2 a 32\left(4 a 20 a 44+a 21 a 43-a 32^{2}\right)}{a 21^{2}}-\frac{a 43 a 32}{a 21}\right.$

$\left.\left.-\frac{8 a 31 a 44}{a 21}\right) u\right) y^{5}$

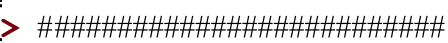

$X 0:=\operatorname{mtaylor}(\operatorname{subs}(v=0, X 1),[y, u], 4)$

$X 0:=2 y u-\frac{a 32 y^{2} u}{a 21}-\frac{4 a 44 y^{3}}{a 21}$

(8)

$X 01:=$ mtaylor $\left(\right.$ mtaylor $\left.\left(\operatorname{subs}\left(y=y-\frac{\frac{(-a 32)}{a 21}}{3\left(-\frac{4 a 44}{a 21}\right)} \cdot u, X 0\right),[y], 4\right), u, 2\right)$

$$
X 01:=-\frac{4 a 44 y^{3}}{a 21}+2 y u
$$

$Y 0:=\operatorname{mtaylor}(\operatorname{subs}(v=0, Y),[y, u], 5)$ 


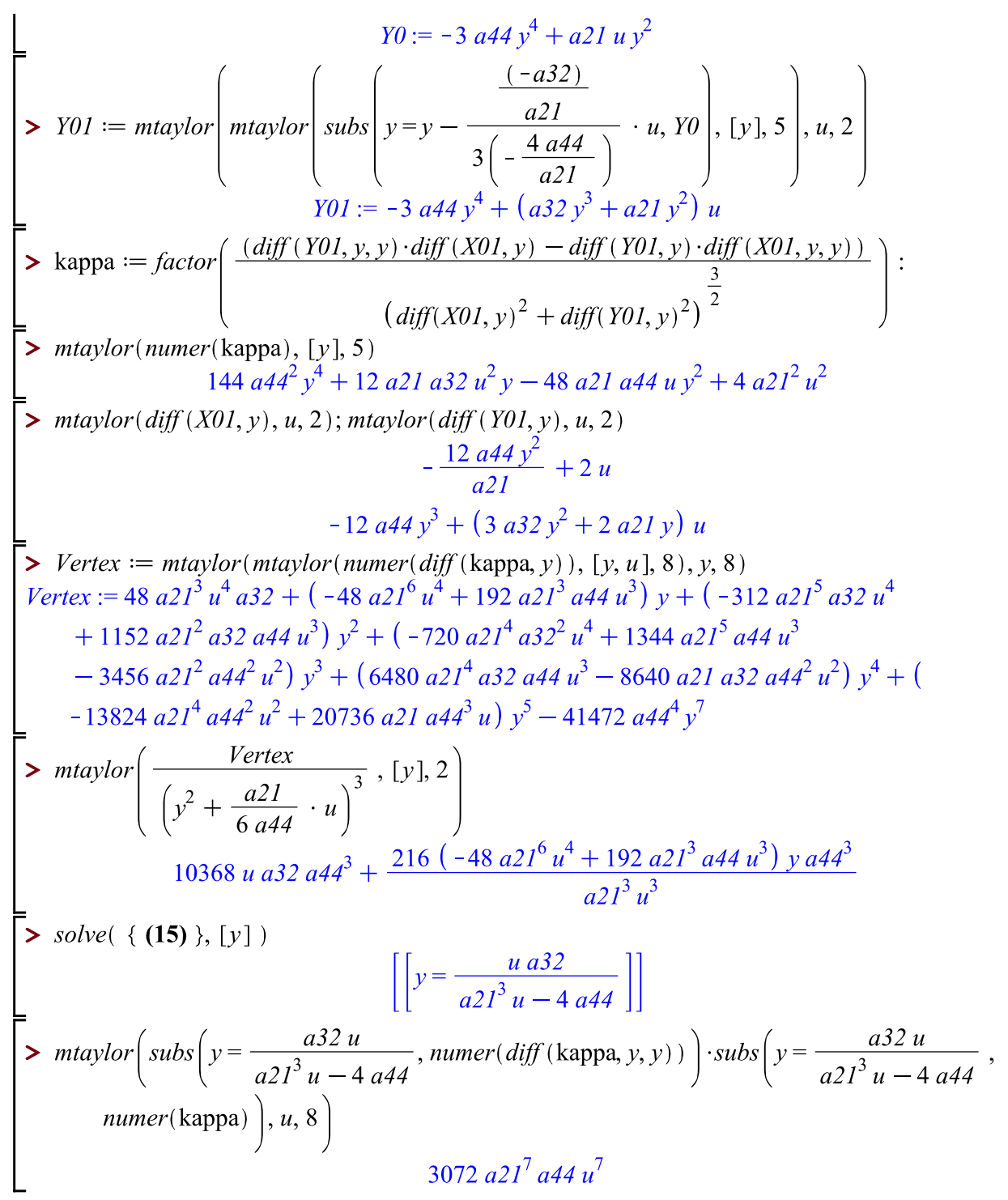




\section{MAPLE CODES IN THE PROOF OF THEOREM 5.7}

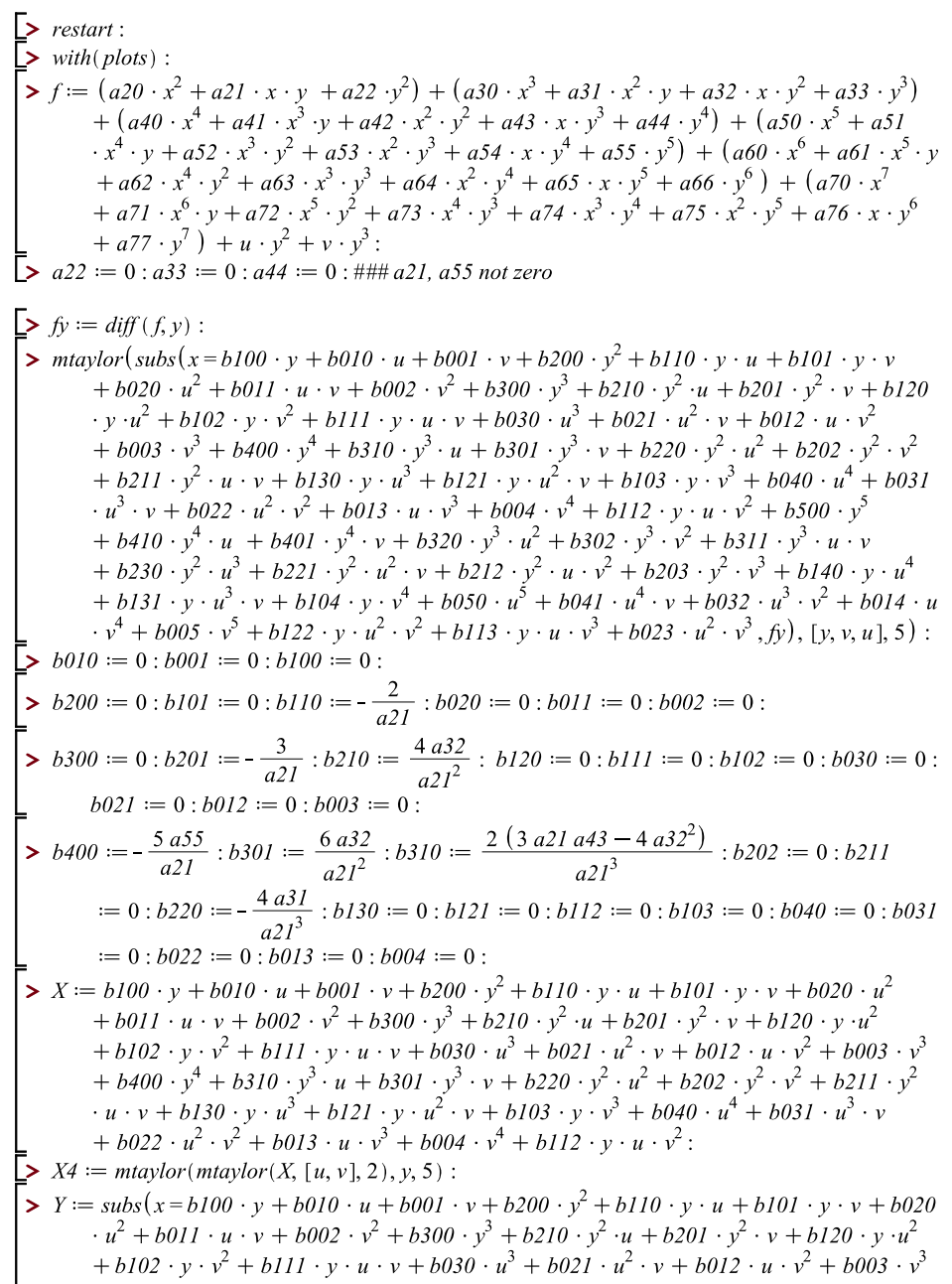




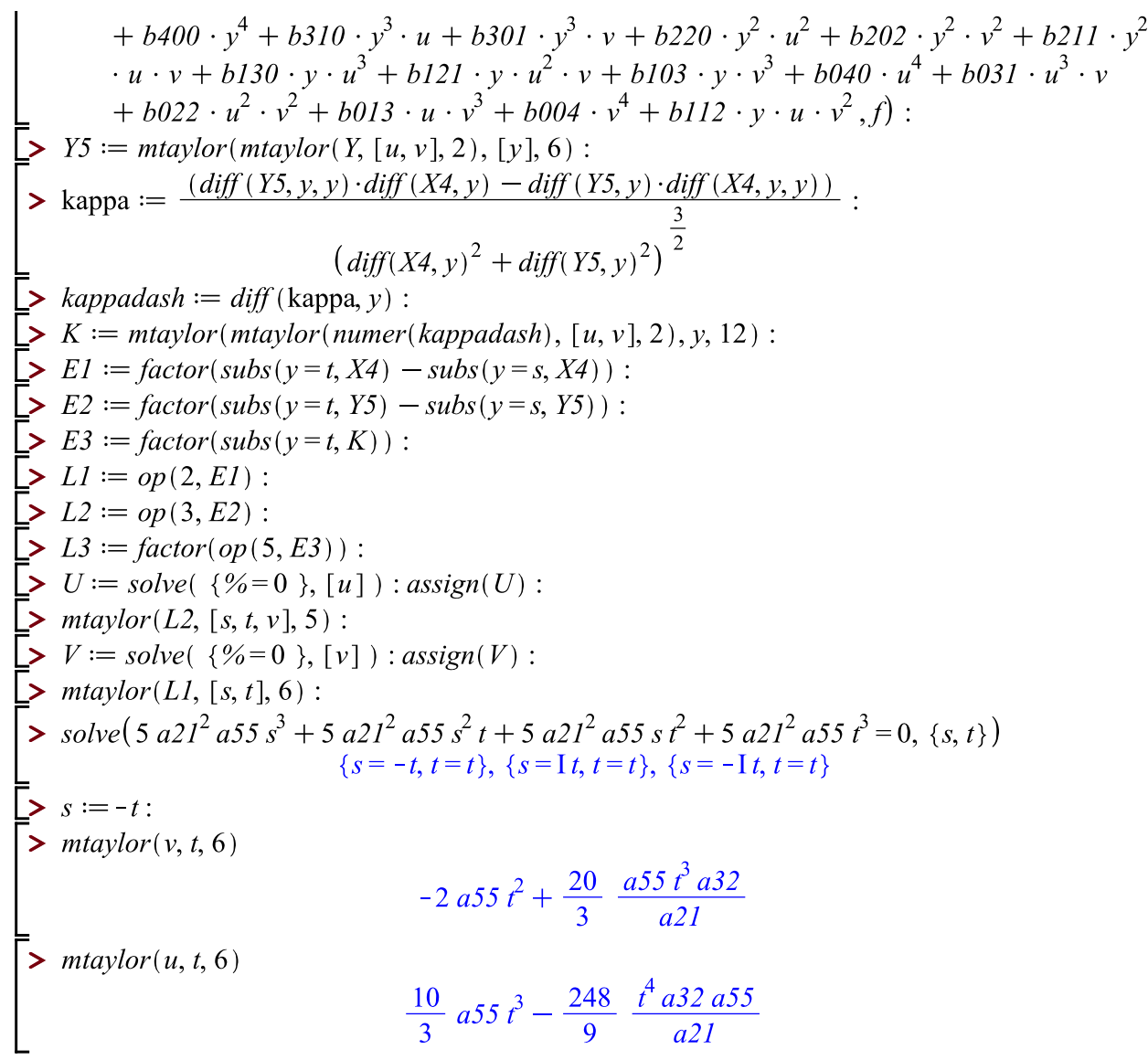

Figure D.1 - The $V T$-stratum in the bifurcation of the profile at a butterfly singularity. 


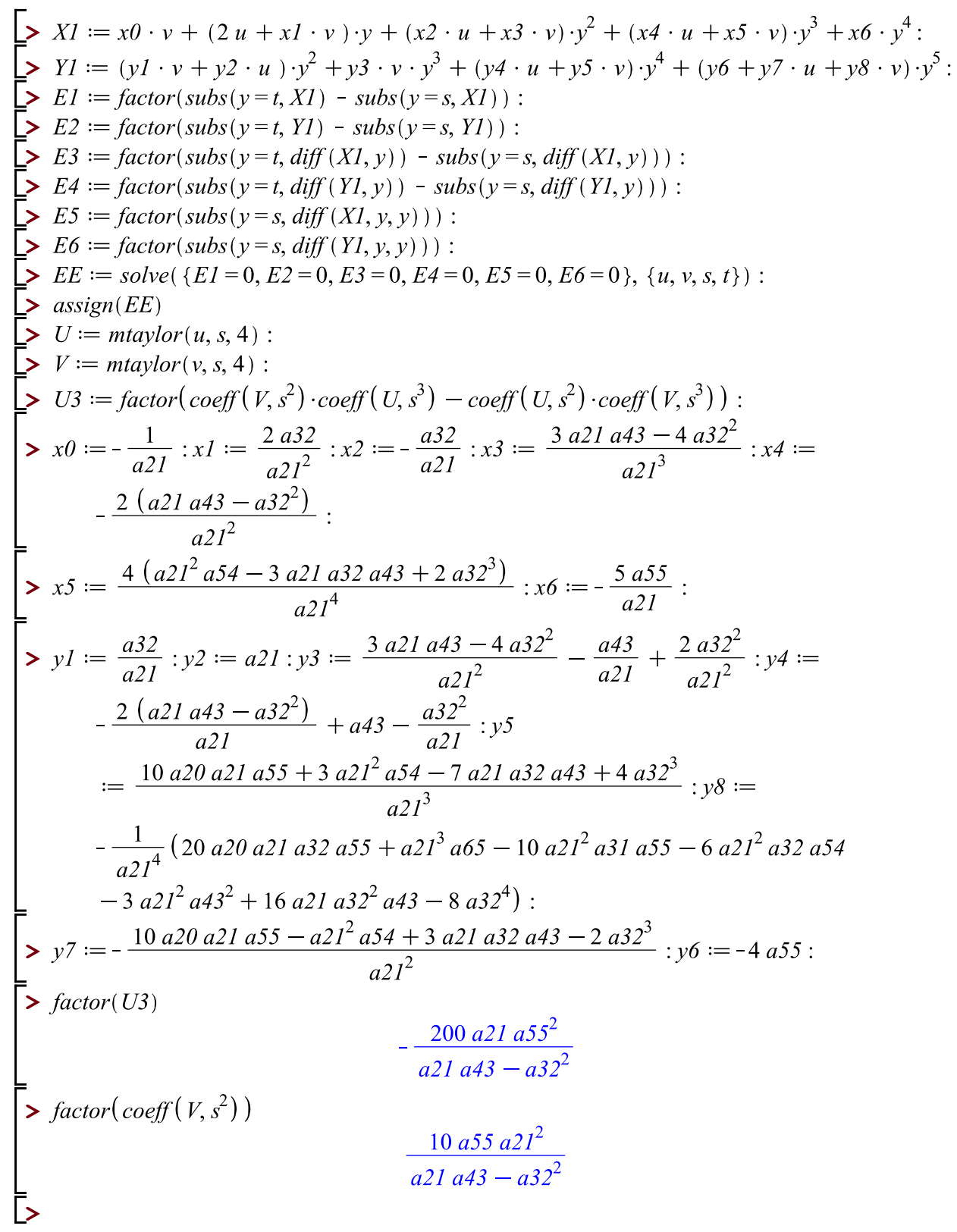

Figure D.2 - The $F C$-stratum in the bifurcation of the profile at a butterfly singularity. 


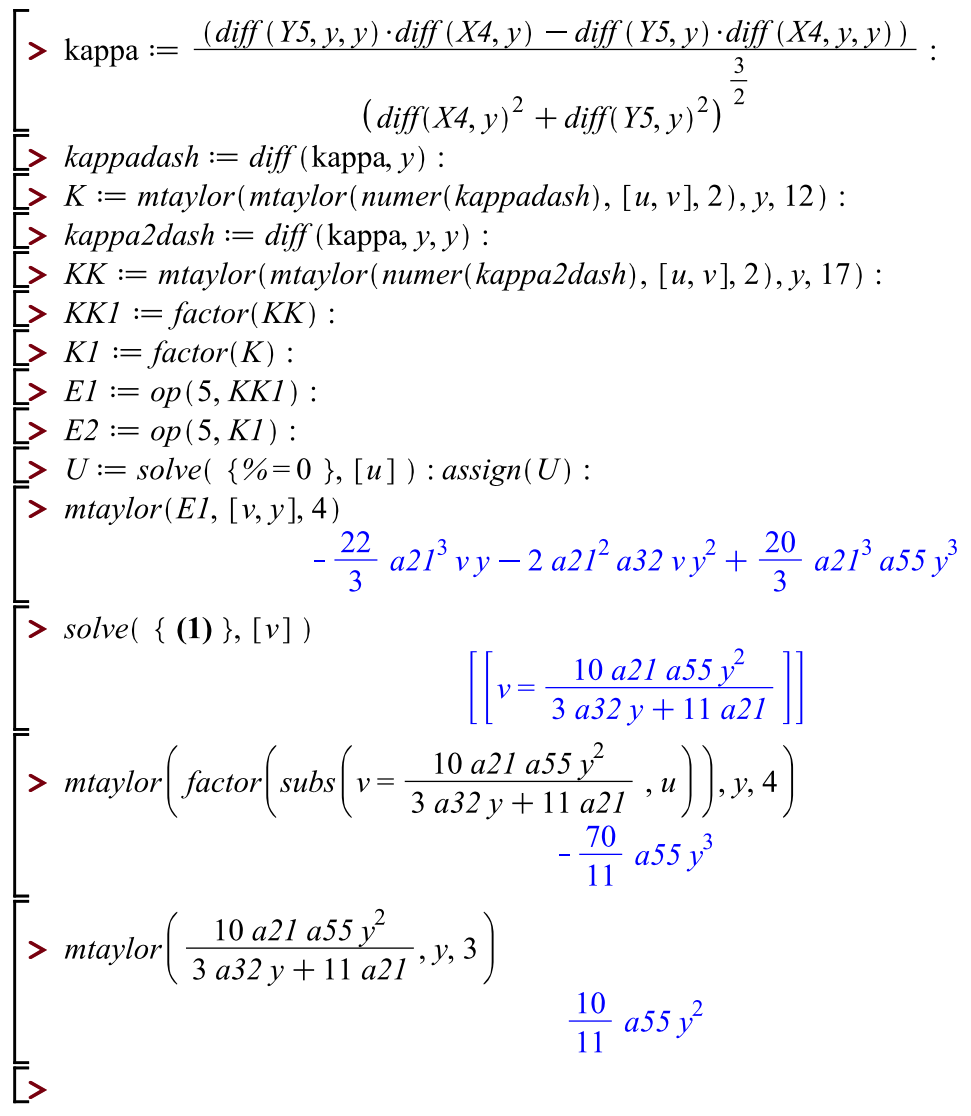

Figure D.3 - The $S V$-stratum in the bifurcation of the profile at a butterfly singularity. 